\title{
Inclusive Jet Production Studies at the Tevatron using the CDF Detector ${ }^{1}$
}

\author{
Olga Norniella Francisco \\ Universitat Autònoma de Barcelona \\ Departament de Física \\ Edifici Cn E-08193 Bellaterra (Barcelona)
}

January 2007

supervised by

Mario Martínez Pérez

IFAE

Edifici Cn E-08193 Bellaterra (Barcelona)

${ }^{1}$ Ph.D. Dissertation 
The studies presented in this thesis led to the following publications:

- Measurement of the Inclusive Jet Cross Section using the $k_{T}$ Algorithm in $p \bar{p}$ Collisions at $\sqrt{s}=1.96$ TeV, Phys. Rev. Lett. 96, 122001 (2006).

- Measurement of the Inclusive Jet Cross Section using the $k_{T}$ Algorithm in $p \bar{p}$ Collisions at $\sqrt{s}=1.96 \mathrm{TeV}$ with the CDF II Detector, submitted to PRD (2007), hep-ex/0701051. 


\section{Acknowledgments}

Many people have helped me, in very different ways, to start and finish this Ph.D. thesis. I would like to properly say, "Thanks!", to all of them below. Anyway, considering that I am a person of few words, I am not sure that I will express all my gratitude although I will try my best.

First of all, I would like to thank Mario Martínez for his guidance. I have been one of his first graduate students, and despite this, he has done an excellent job guiding my work day by day. Part of the success of this Ph.D. is based on his experience in the CDF collaboration, his thorough knowledge of QCD and his strong personality. Without a doubt, I know that my future research will be influenced by our collaboration. Another person important for the analysis presented in this thesis has been Regis Lefèvre, "my postdoc". In addition to being a very meticulous physicist, he has been a great co-worker, who has patiently spent hours answering my questions.

I am also grateful to the CDF collaboration. In particular, I want to thank Jacobo Konigsberg and Rob Roser for their leadership as spokespersons. Their efforts, together with previous spokespersons, have ensured the continued success of the CDF experiment. In addition, and more importantly, they have offered a personal, friendly, and easy going environment for all CDF people making the collaboration into a big family, of which I am happy to be a member.

The person responsible for my entry into the exciting adventure of the research world is Enrique Fernández. Thanks for that! As a teacher at the Universitat Autònoma de Barcelona, he was the first to attract me to the field of experimental high energy physics and, once I graduated, he gave me the opportunity to be part of IFAE. At IFAE, I was welcomed by Matteo Cavalli-Sforza, who was my Master Thesis adviser while working in the ATLAS collaboration. I would like to express my gratitude to Matteo for his dedication and support, it has been a pleasure to work with him, a person with such a strong desire to learn new things every day. Coming to other seniors at IFAE, I do not want to forget Mokhtar, who is always very considerate toward all people, in particular with students. And to finish with the IFAE community, I want to thank all the people who have 
cheered my up when I was writing my thesis at IFAE, especially Laia, Cristina, Ramon, Ester Aliu, Carolina, David, Toni, Lluís, Pere and Jordi Nadal.

Ninety-nine percent of the thesis has been done at Fermilab, and even though I have been spending almost all my time at the lab, when I think about Fermilab I am reminded a lot of the great moments shared with the "Spanish mafia". First, thanks to Oscar, Aran, Jonatan, and Guillelmo who admitted me into their life without hesitation. After my arrival in Chicago, the Spanish community started to expand...Kike, Xavi, Oriol, Ernest, Cristobal, and more recently, Ignacio, Bárbara, Bruno, Roberto, and Miguel landed in Illinois as well. I send them a lot of "petonets" as gratitude for the moments we spent together. I would like to add a couple of things. First, to say to Cristobal and Kike that I promise to take care of "Olga the walrus". And second, send my deepest appreciation to Xavi, who has been more than a house-mate and office-mate. He has been a really good friend, being my confidant and always giving me sincere advice.

Finally, I would like to mention something about the people who are most important in my life. My parents and my sister, who have always supported and respected my decisions, giving me the freedom to live my life as I choose, and not complaining because I have selected to stay in a city more than $7000 \mathrm{~km}$ far away from "home". My last words are dedicated to Len and the "boys", who like taking care of me. I am really happy to have met them and I hope they will be close to me in my next American adventure. 


\section{Contents}

$\begin{array}{ll}\text { List of Figures } & \mathbf{v}\end{array}$

$\begin{array}{ll}\text { List of Tables } & \text { ix }\end{array}$

1 Introduction 1

2 QCD and Jets in $p \bar{p}$ collisions $\quad 3$

2.1 The Standard Model . . . . . . . . . . . . . . . . . . . . 3

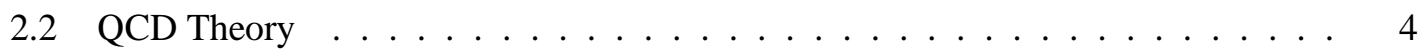

2.3 Deep Inelastic scattering and the Quark Parton Model . . . . . . . . . . . . . . . 6

2.4 Hadron-hadron processes and QCD Factorization $\ldots \ldots \ldots$

2.5 Parton Distribution Functions . . . . . . . . . . . . . . . . . . . . . 14

2.5.1 MRST and CTEQ parametrizations . . . . . . . . . . . . . 14

2.5 .2 PDFs uncertainties $\ldots \ldots \ldots \ldots \ldots$

$2.6 \mathrm{pQCD}$ calculation for jet production $\ldots \ldots \ldots \ldots \ldots$

2.7 Beyond NLO: MonteCarlo programs . . . . . . . . . . . . . . . 20

2.7.1 Parton shower. . . . . . . . . . . . . . . 21

2.7.2 Hadronization . . . . . . . . . . . . . . . . . . . . . 22

2.8 Jet Phenomenology . . . . . . . . . . . . . . . . . . . . . . . . . . 24

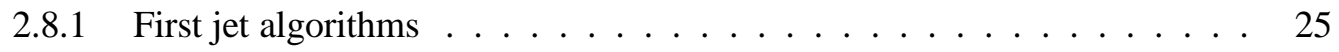


2.8 .2 Cone-based algorithms . . . . . . . . . . . . . . . 26

2.8 .3 The cluster-type algorithms . . . . . . . . . . . . . . . . 29

2.9 Jet Physics at Hadron Colliders . . . . . . . . . . . . . . . . . . . . . 32

3 CDF at Fermilab 35

3.1 The Tevatron Collider . . . . . . . . . . . . . . . . . 35

3.2 CDF Run II detector $\ldots \ldots \ldots \ldots$. . . . . . . . . . . . . . . 39

3.2.1 Tracking and Time of Flight systems . . . . . . . . . . . . . . . 40

3.2 .2 Calorimeter system . . . . . . . . . . . . . . . . 43

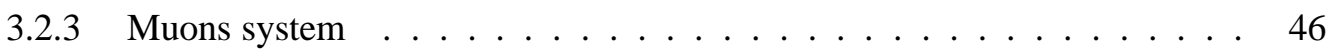

3.3 Luminosity Measurement . . . . . . . . . . . . . . . . . . . . . 46

$3.3 .1 \quad$ CLC detector . . . . . . . . . . . . . . . . 46

3.3.2 Measurement of the luminosity _. . . . . . . . . . . . . 47

3.4 Trigger and Data Acquisition . . . . . . . . . . . . . . . . . 48

3.4 .1 Level 1 trigger $\ldots \ldots \ldots \ldots \ldots \ldots \ldots \ldots$

3.4 .2 Level 2 trigger . . . . . . . . . . . . . . . . 52

3.4 .3 Level 3 trigger $\ldots \ldots \ldots \ldots \ldots \ldots$

3.5 Data Quality Monitoring . . . . . . . . . . . . . . . . . 53

4 Inclusive Jet Cross Section Measurement 55

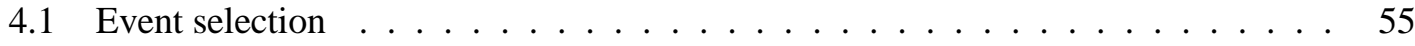

$4.1 .1 \quad$ Run selection . . . . . . . . . . . . . . . . . 55

4.1 .2 Jet selection criteria . . . . . . . . . . . . . . . 56

4.2 Trigger Study . . . . . . . . . . . . . . . . . . . . 59

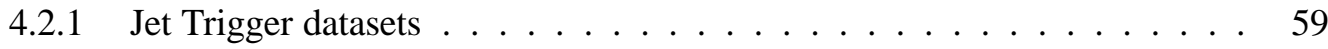

4.3 Pile-up Correction . . . . . . . . . . . . . . . . . . 66

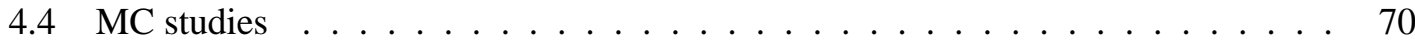


4.4.1 Raw variables comparison . . . . . . . . . . . . . 70

4.4 .2 Bisector Method . . . . . . . . . . . . . . . 76

4.4.2.1 Correction for underestimated resolution in the simulation . . . 81

4.4.2.2 Correction for overestimated resolution in the simulation . . . 82

4.4 .3 Dijet Balance . . . . . . . . . . . . . . . 86

4.4 .4 Summary of $\mathrm{MC}$ corrections $\ldots \ldots \ldots \ldots$

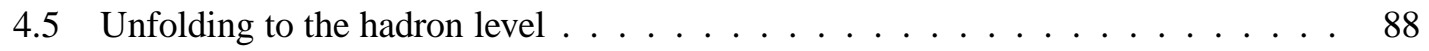

4.5.1 Average $p_{T}^{\text {jet }}$ correction . . . . . . . . . . . . . . 90

4.5 .2 Unfolding Procedure . . . . . . . . . . . . . . . . . 91

4.5.2.1 Resolution correction . . . . . . . . . . . . 92

4.6 Systematic Uncertainties . . . . . . . . . . . . . . . . . . . . . . . . . . . 94

5 Discussion of the Results 101

$5.1 \quad$ NLO calculations . . . . . . . . . . . . . . . . . . . . . . . 101

5.1.1 Hadronization and Underlying event correction . . . . . . . . . . 103

5.2 Inclusive Jet Production results . . . . . . . . . . . . . . . . . . . 106

6 Summary and Conclusions $\quad 111$

$\begin{array}{ll}\text { A Data Quality Monitoring system } & 113\end{array}$

A.1 Introduction . . . . . . . . . . . . . . . . . 113

A.2 Online DQM system . . . . . . . . . . . . . . . . . 113

A.2.1 Check list . . . . . . . . . . . . . . . . . . 115

A.2.2 DQM Monitor . . . . . . . . . . . . . . . . . . . 116

$\begin{array}{llr}\text { B Jet Shapes Studies } & 119\end{array}$

B.1 Jet Shapes measurements . . . . . . . . . . . . . . . . . . . . . . 119

$\begin{array}{llr}\text { C Results Tables } & 121\end{array}$ 
Bibliography 


\section{List of Figures}

2.1 The value of the running coupling constant, $\alpha_{S}$, as a function of the energy scale $\mathrm{E} 5$

2.2 Schematic view of a deep inelastic scattering reaction $\ldots \ldots \ldots \ldots$

2.3 Kinematic range accessible by the Tevatron in Run I . . . . . . . . . . . . . . . . 7

2.4 Example of parton distribution functions $\ldots \ldots \ldots \ldots$

2.5 The processes related to the lowest order QCD splitting functions . . . . . . . 10

2.6 Structure function $F_{2}$ measurements from ZEUS experiment $\ldots \ldots \ldots \ldots$

2.7 CDF Run I Cross section results compared to NLO calculations, using CTEQ3M PDFs set . . . . . . . . . . . . . . . . . . . . . 15

2.8 CDF Run I Cross section results compared to NLO calculations, using CTEQ4 PDFs sets . . . . . . . . . . . . . . . . . . . . 16

2.9 Uncertainty on gluon and u-quark PDFs $\ldots \ldots \ldots \ldots \ldots$

2.10 Diagrams for jet production at LO in a hadron collider . . . . . . . . . . 19

2.11 The subprocesses contributions to jet production in the central and forward rapidity regions . . . . . . . . . . . . . . . . . . . . . . 19

2.12 Parton shower with string and cluster hadronization models . . . . . . . . . . . . 24

2.13 Example of an overlapping situation between two stable cones defined with a cone-based algorithm . . . . . . . . . . . . . . . . . 28

2.14 An illustration of infrared sensitivity in cone jet clustering . . . . . . . . . . 29

2.15 An illustration of collinear sensitivity in jet reconstruction _ . . . . . . . . 29 
2.16 An illustration of the final jet geometry using the cone or the $k_{T}$ algorithm . . . . 31

2.17 Example on the same event of jets found using different algorithms . . . . . . . 32

3.1 The Tevatron Collider Chain at Fermilab . . . . . . . . . . . . . . . 36

3.2 Tevatron Collider Run II Integrated Luminosity . . . . . . . . . . . . . 38

3.3 Tevatron Collider Run II Peak Luminosity . . . . . . . . . . . . . . . . . . . 38

3.4 Isometric view of the CDF Run II detector $\ldots \ldots \ldots$. . . . . . . . . . 39

$3.5 r \times \eta$ side view of the CDF Run II detector . . . . . . . . . . . . . . 40

3.6 Layout of wire planes on a COT endplate . . . . . . . . . . . . . 41

3.7 Layout of wires in a COT supercell . . . . . . . . . . . . . . . . 42

3.8 The CDF II tracker layout showing the different subdetector systems . . . . . . . 43

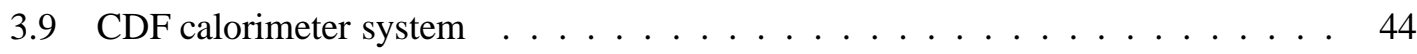

3.10 Block diagram showing the global trigger and DAQ systems at CDF II . . . . . . 49

3.11 Block diagram showing the Level 1 and Level 2 trigger system . . . . . . . . 50

4.1 Missing $E_{T}$ significance cut study $\ldots \ldots \ldots \ldots$. . . . . . . . . 57

4.2 Efficiency of the primary vertex and the missing $E_{T}$ significance cuts $\ldots \ldots 57$

4.3 Dijet balance studies using data and PYTHIA-Tune A MC . . . . . . . . . 58

4.4 Trigger flow for the jet triggers $\ldots \ldots \ldots \ldots \ldots$

4.5 The $\mathrm{L} 2$ cluster finder algorithm . . . . . . . . . . . . . . . . . 60

4.6 Trigger efficiency versus $p_{T, C A L}^{\text {jet }}$ studies I . . . . . . . . . . . . 62

4.7 Trigger efficiency versus $p_{T, C A L}^{\text {jet }}$ studies II $\ldots \ldots \ldots \ldots \ldots$

4.8 Global trigger efficiency versus $p_{T, C A L}^{j e t} \ldots \ldots \ldots \ldots \ldots \ldots$. . . . . . . 64

4.9 Measured $p_{T, C A L}^{j e t}$ distribution in the five rapidity regions . . . . . . . . . . 64

4.10 Pile-up correction studies I . . . . . . . . . . . . . . . . 67

4.11 Pile-up correction studies II . . . . . . . . . . . . . . . . . 68

4.12 Pile-up corrections in the five rapidity regions $\ldots \ldots \ldots$. . . . . . . . . . 69 
4.13 Comparison between data and $\mathrm{MC}$ for some jet variables in the rapidity region

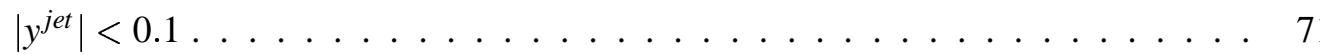

4.14 Comparison between data and MC for some jet variables in the rapidity region

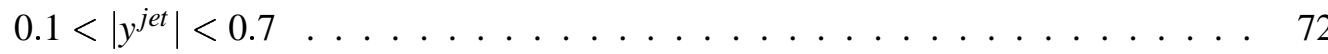

4.15 Comparison between data and MC for some jet variables in the rapidity region

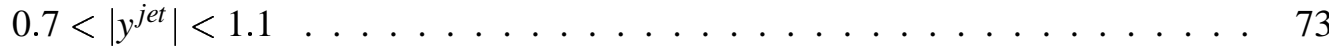

4.16 Comparison between data and MC for some jet variables in the rapidity region

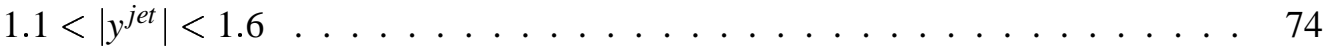

4.17 Comparison between data and $\mathrm{MC}$ for some jet variables in the rapidity region

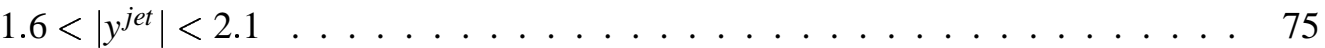

4.18 Illustration of the bisector method variables $\ldots \ldots \ldots \ldots$

4.19 Bisector method study result: $\sigma_{/ /}$in DATA and MC $\ldots \ldots \ldots$

4.20 Bisector method study result: $\sigma_{/ /}$DATA / MC . . . . . . . . . . 78

4.21 Bisector method study result: $\sigma_{P E R P}$ in DATA and MC . . . . . . . . . . . 79

4.22 Bisector method study result: $\sigma_{P E R P}$ DATA / MC $\ldots \ldots \ldots$

4.23 Bisector method study result: $\sigma_{D}$ in DATA and MC $\ldots \ldots \ldots$

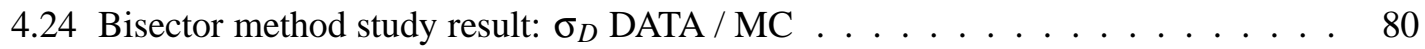

$4.25 p_{T}^{j e t}$ resolution obtained from the MC for jets in the different regions . . . . . . 82

4.26 Bisector method study results for jets in the region $0.7<\left|y^{j e t}\right|<1.1$ after corrections 83

4.27 Bisector method study results for jets in the region $1.6<\left|y^{j e t}\right|<2.1$ after corrections 84

4.28 Bisector method study results for jets in the region $1.1<\left|y^{\text {jet }}\right|<1.6$ after corrections 85

4.29 Dijet study results for jets in the different rapidity regions . . . . . . . . . . . 87

4.30 Weighting procedure $\ldots \ldots \ldots \ldots \ldots \ldots$

4.31 Average $p_{T}$ correction in the different rapidity regions . . . . . . . . . . . 90

4.32 Bin-by-bin unfolding factors vs $p_{T, C O R}^{\text {jet }}$ obtained using reweighted PYTHIA-Tune A 91

4.33 Resolution correction factors and modified unfolding factors for jets in the region

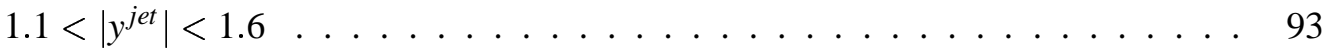


4.34 Systematic uncertainty associated to the uncertainty on the Jet Energy Scale . . . 94

4.35 Systematic uncertainty associated to the uncertainty on the Dijet Method . . . . . 96

4.36 Systematic uncertainty associated to the uncertainty on the Dijet Correction . . . 96

4.37 Systematic uncertainty associated to the uncertainty on the resolution $\ldots$. . . . 97

4.38 Systematic uncertainty associated to the uncertainty on the $p_{T}$ spectra $\ldots . . .98$

4.39 Systematic uncertainty associated to the uncertainty on the unfolding procedure . 98

4.40 Systematic uncertainty associated to the uncertainty on the pile-up correction . . 99

4.41 Total systematic uncertainty . . . . . . . . . . . . . . 100

5.1 Fractional difference between the different PDFs sets and the standard prediction 102

5.2 NLO systematic uncertainties . . . . . . . . . . . . . . . . . . . . 103

5.3 Global Underlying Event/Hadronization correction and its factorization . . . . . 105

5.4 Parton to hadron level corrections as obtained from PYTHIA-Tune A and HERWIG105

5.5 Inclusive jet cross sections measured compared to pQCD NLO calculations . . . 106

5.6 Parton to hadron level corrections and systematic uncertainties applied to the pQCD calculations . . . . . . . . . . . . . . . . . 107

5.7 Ratio between the measurements and the pQCD calculations . . . . . . . . 108

5.8 Inclusive jet cross sections measured for $\mathrm{D}=0.5$ and $\mathrm{D}=1.0 \ldots \ldots$. . . . . 109

A.1 DQMon panel . . . . . . . . . . . . . . . . . . . . 117

B.1 Illustration of the integrated jet shape definition . . . . . . . . . . . . 119

B.2 Measured integrated jet shape compared to different MC predictions . . . . . . 120 


\section{List of Tables}

2.1 The Standard Model elementary particles and forces carried $\ldots \ldots \ldots \ldots$

2.2 LO jet production matrix elements squared $\bar{\Sigma}|M|^{2} / g^{4} \ldots \ldots \ldots \ldots$

3.1 Accelerator parameters for Run II configuration . . . . . . . . . . . . . . . . 37

3.2 CDF II Calorimeter subsystems and characteristics $\ldots \ldots \ldots$

$4.1 p_{T, C A L}^{j e t}$ thresholds for the different datasets and rapidity ranges $\ldots \ldots \ldots 61$

4.2 $p_{T}^{j e t}$ bins and the corresponding datasets used for the measured $p_{T, C A L}^{j e t}$ distribution in the different rapidity regions . . . . . . . . . . . . . . 65

$4.3 \varepsilon$ values and their uncertainties for each rapidity region $\ldots \ldots$. . . . . . . . 69

4.4 Corrections applied to the MC coming from the bisector method and dijet balance studies . . . . . . . . . . . . . . . . . . . . 88

4.5 PYTHIA-Tune A MC samples used in each $p_{T, C O R}^{\text {jet }}$ bin to evaluate the unfolding corrections in the different rapidity regions . . . . . . . . . . . . . . 92

C.1 Systematic uncertainties (in percentage) on the measured inclusive jet differential cross section as a function of $p_{T}^{j e t}$ for jets in the region $\left|y^{j e t}\right|<0.1 \ldots \ldots 121$

C.2 Systematic uncertainties (in percentage) on the measured inclusive jet differential cross section as a function of $p_{T}^{\text {jet }}$ for jets in the region $0.1<\left|y^{\text {jet }}\right|<0.7 \ldots \ldots .122$

C.3 Systematic uncertainties (in percentage) on the measured inclusive jet differential cross section as a function of $p_{T}^{\text {jet }}$ for jets in the region $0.7<\left|y^{\text {jet }}\right|<1.1 \ldots 123$ 
C.4 Systematic uncertainties (in percentage) on the measured inclusive jet differential cross section as a function of $p_{T}^{\text {jet }}$ for jets in the region $1.1<\left|y^{j e t}\right|<1.6 \ldots 124$

C.5 Systematic uncertainties (in percentage) on the measured inclusive jet differential cross section as a function of $p_{T}^{\text {jet }}$ for jets in the region $1.6<\left|y^{j e t}\right|<2.1 \ldots 125$

C.6 Measured inclusive jet differential cross section as a function of $p_{T}^{\text {jet }}$ for jets in the region $\left|y^{j e t}\right|<0.1$ and the parton-to-hadron correction factors . . . . . . 126

C.7 Measured inclusive jet differential cross section as a function of $p_{T}^{j e t}$ for jets in the region $0.1<\left|y^{j e t}\right|<0.7$ and the parton-to-hadron correction factors $\ldots$.

C.8 Measured inclusive jet differential cross section as a function of $p_{T}^{\text {jet }}$ for jets in the region $0.7<\left|y^{j e t}\right|<1.1$ and the parton-to-hadron correction factors . . . .

C.9 Measured inclusive jet differential cross section as a function of $p_{T}^{j e t}$ for jets in the region $1.1<\left|y^{j e t}\right|<1.6$ and the parton-to-hadron correction factors $\ldots$.

C.10 Measured inclusive jet differential cross section as a function of $p_{T}^{\text {jet }}$ for jets in the region $1.6<\left|y^{j e t}\right|<2.1$ and the parton-to-hadron correction factors $\ldots$.

C.11 Measured inclusive jet differential cross section as a function of $p_{T}^{\text {jet }}$ for jets in the region $0.1<\left|y^{\text {jet }}\right|<0.7$ using $D=0.5$ and the parton-to-hadron correction

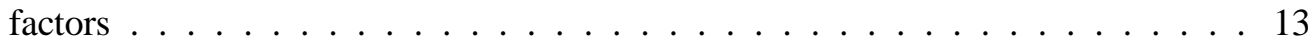

C.12 Measured inclusive jet differential cross section as a function of $p_{T}^{\text {jet }}$ for jets in the region $0.1<\left|y^{\text {jet }}\right|<0.7$ using $D=1.0$ and the parton-to-hadron correction

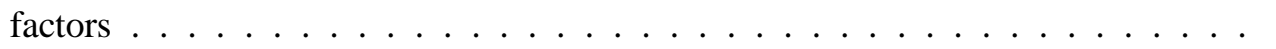

C.13 Systematic uncertainties (in percentage) on the measured inclusive jet differential cross section as a function of $p_{T}^{\text {jet }}$, for jets in the region $0.1<\left|y^{\text {jet }}\right|<0.7$ and using $D=0.5 \ldots \ldots \ldots \ldots \ldots \ldots \ldots \ldots$

C.14 Systematic uncertainties (in percentage) on the measured inclusive jet differential cross section as a function of $p_{T}^{j e t}$, for jets in the region $0.1<\left|y^{j e t}\right|<0.7$ and

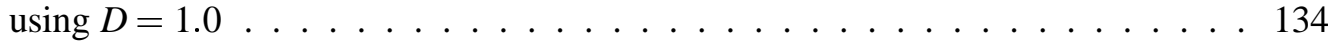




\section{Chapter 1}

\section{Introduction}

Quantum Chromodynamics (QCD) is the gauge theory that governs the strong interactions between quarks and gluons inside hadrons like, for example, protons and neutrons. It shows two well established characteristics, related to the non-abelian nature of the theory, that dominate its phenomenology: asymptotic freedom and color confinement. The dependence of the strong coupling, $\alpha_{s}\left(Q^{2}\right)$, with the hard scale is such that it decreases with decreasing the distance between partons. This allows to perform precise theoretical calculations at large energy transfer (short distances) using perturbative QCD (pQCD). In the other hand, the strength of the interaction increases with the distance between partons and thus colored quarks and gluons are forced to be confined inside colorless hadrons.

At the Tevatron at Fermilab, protons and antiprotons collide at very high energy. In those collisions, collimated jets of hadrons are produced along the direction of struck quarks and gluons in the final state. The measurement of the inclusive jet production cross section for central jets constitutes one of the pillars of the jet physics program since it provides a stringent test of pQCD predictions over almost nine order of magnitude. Thanks to the increase in the center-of-mass energy at the Tevatron in Run II (from 1.8 to $1.96 \mathrm{TeV}$ ) the jet production rate is multiplied by a factor of five for jets with transverse momentum, $p_{T}^{\text {jet }}$, higher than $600 \mathrm{GeV}$, and the new measurements are extended the $p_{T}^{\text {jet }}$ coverage by $150 \mathrm{GeV}$ compared to Run I. In addition, the CDF experiment explores new algorithms to define jets, following the theoretical work that indicates that the cone-based jet algorithm employed in Run I is not infrared safe and compromises a future meaningful comparison with a pQCD calculation at NNLO.

The Tevatron jet data has been used in the past to determine the gluon distribution in the proton 
at high $\mathrm{x}$. Jet measurements at large rapidities are important because they constrain the gluon density in a region in $p_{T}^{\text {jet }}$ where no effect from new physics is expected.

The hadronic final states at $2 \mathrm{TeV}$ are characterized by the presence of soft underlying emissions, usually denoted as underlying event, in addition to highly energetic jets of hadrons coming from the hard interaction. The underlying event contains contributions from initial- and final-state soft gluon radiation, secondary semi-hard partonic interactions and interactions between the proton and anti-proton remnants that cannot be computed following perturbation theory. These processes must be approximately described using phenomenological models tuned to the data. Hence, a proper understanding of this underlying contribution is crucial to reach the desired precision in the measured cross sections.

This $\mathrm{PhD}$. Thesis presents a measurement of the inclusive jet production cross section using the new data collected by the CDF experiment in Run II. The longitudinally invariant $k_{T}$ algorithm, infrared safe to all orders in $\mathrm{pQCD}$, has been used in order to search for jets in the final state. The measurement is compared to pQCD NLO calculations where non-perturbative effects from the underlying event and the fragmentation of partons into jets of hadrons have been taken into account.

The present work is organized as follows: Chapter 2 gives an introduction to the theory of strong interaction and describes the main concepts of jet physics. Chapter 3 contains a description of the Tevatron accelerator and the CDF detector. The details of the analysis are explained in Chapter 4. Chapter 5 presents the final results and Chapter 6 is devoted to summary and conclusions.

At the end of the document three appendixes are included. Appendix A describes the Data Quality Monitoring system implemented at CDF. The Appendix B presents Jet Shapes measurements published in Run II from CDF. These precise measurements of the jet internal structure, sensitive to non-perturbative effects, are important to guarantee a precise comparison between the jet cross section measurements and the NLO calculations. Finally, the tables in Appendix C summarize the results presented in this $\mathrm{PhD}$. Thesis. 


\section{Chapter 2}

\section{QCD and Jets in $p \bar{p}$ collisions}

At present the most successful way to describe the measurements in high energy experiments is summarized in the Standard Model. In this chapter a discussion about this model is presented, including how it describes the interaction between elementary particles and the formation of hadronic jets. The chapter finalizes with a discussion about jet physics at hadron colliders.

\subsection{The Standard Model}

The Standard Model is an attempt to describe the properties and interactions of elementary particles. There are four known fundamental forces which govern the interaction of matter in our universe: gravity, electromagnetism, weak and strong interactions. The Standard Model groups the electromagnetic, weak and strong interactions in a quantum field theory based on group symmetries. These forces are mediated by the so-called carrier particles, which have integer spin (1), obey Bose-Einstein statistics and are called gauge bosons. The electromagnetic force is mediated via the exchange of massless photons, $\gamma$. The weak force is transmitted by exchange of three massive intermediate vector bosons, the $W^{ \pm}$and $Z$. The strong force is mediated via eight massless gluons.

Besides gauge bosons, one observes a second type of fundamental particles. These particles have half-integer spin and are called fermions. They follow Fermi-Dirac statistics and are constrained by the Pauli exclusion principle. There are two fundamentally different types of fermions: leptons and quarks. They both interact electroweakly, but only the quarks feel the strong force. The lepton 
family is constituted by electrons (e), muons $(\mu)$ and taus $(\tau)$ and their associated neutrinos, $v_{e}$, $v_{\mu}$ and $v_{\tau}$, respectively. There are six massive quarks, or flavors: up (u), down (d), strange (s), charm (c), bottom (b) and top (t). Out of the six quarks three of them (u,c,t) have a charge of $Q=2 / 3$ and are also known as up-type quarks. The other three quarks $(\mathrm{d}, \mathrm{s}, \mathrm{b})$ are down-type and carry an electric charge of $Q=-1 / 3$. The three up and down-type quarks can be paired to form three "sets" of quarks which have increasing mass. Each "set" of quarks is called a generation, or family. Table 2.1 summarizes the Standard Model forces carried and elementary particles.

\begin{tabular}{|c|ccc|}
\hline $\begin{array}{c}\text { Gauge Bosons } \\
\text { Mediator Interaction }\end{array}$ & $\begin{array}{c}W^{ \pm}, Z^{0} \\
\text { Weak }\end{array}$ & $\begin{array}{c}\text { Photon } \\
\text { Electromagnetic }\end{array}$ & $\begin{array}{c}\text { 8 Gluons } \\
\text { Strong }\end{array}$ \\
\hline \hline Quarks & $\left(\begin{array}{c}u \\
d\end{array}\right)$ & $\left(\begin{array}{l}c \\
s\end{array}\right)$ & $\left(\begin{array}{c}t \\
b\end{array}\right)$ \\
\hline Leptons & $\left(\begin{array}{c}v_{e} \\
e\end{array}\right)$ & $\left(\begin{array}{c}v_{\mu} \\
\mu\end{array}\right)$ & $\left(\begin{array}{c}v_{\tau} \\
\tau\end{array}\right)$ \\
\hline
\end{tabular}

Table 2.1: The Standard Model elementary particles and forces carried.

\subsection{QCD Theory}

One of the cornerstones of the Standard Model is Quantum Chromodynamics (QCD) that describes the strong interaction. Following the way opened by QED and Yang-Mills theories, QCD was developed in 1973 [1] in the context of Quantum Field Theory based in SU(3) symmetry group [2]. It is a non-abelian theory and the Lagrangian, that describes the strong interaction of coloured quarks and gluons ${ }^{1}$, is given by:

\footnotetext{
${ }^{1}$ The charge associated with the strong interaction is the colour-charge. The colour property was introduced to quarks satisfied the requirement of Pauli exclusion principle. Posterior experiment results proved the validity of colour hypothesis
} 


$$
\mathcal{L}_{Q C D}=\sum_{\text {flavour }} \bar{q}_{a}\left(i \gamma^{\mu} D_{\mu}-m_{q}\right)_{a b} q_{b}-\frac{1}{4} F_{\alpha \beta}^{A} F_{A}^{\alpha \beta}
$$

where the sum runs over the six different flavors of the quarks. $F_{\alpha \beta}^{A}$ is the field strength tensor derived from the gluon field $A_{\alpha}^{A}$ as,

$$
F_{\alpha \beta}^{A}=\left[\partial_{\alpha} A_{\beta}^{A}-\partial_{\beta} A_{\alpha}^{A}-g f^{A B C} A_{\alpha}^{B} A_{\beta}^{C}\right]
$$

and the indices A,B,C run over the eight colour degrees of freedom of gluon field. $\mathrm{g}$ is the coupling constant, which determines the strength of the interaction between coloured quanta, and $f^{A B C}$ are the structure constants of the SU(3) colour group. The third term in equation 2.2 shows the non-abelian nature of QCD. This term describes the property of interaction between gluons, resulting in the very different behavior of the strong interaction compared to the electromagnetic interaction. This self-coupling is the reason for the strong coupling constant,$\alpha_{s}=\frac{g^{2}}{4 \pi}$, is large at small energies (large distances) and decreases at high energies (small distance) as is shown in figure 2.1.

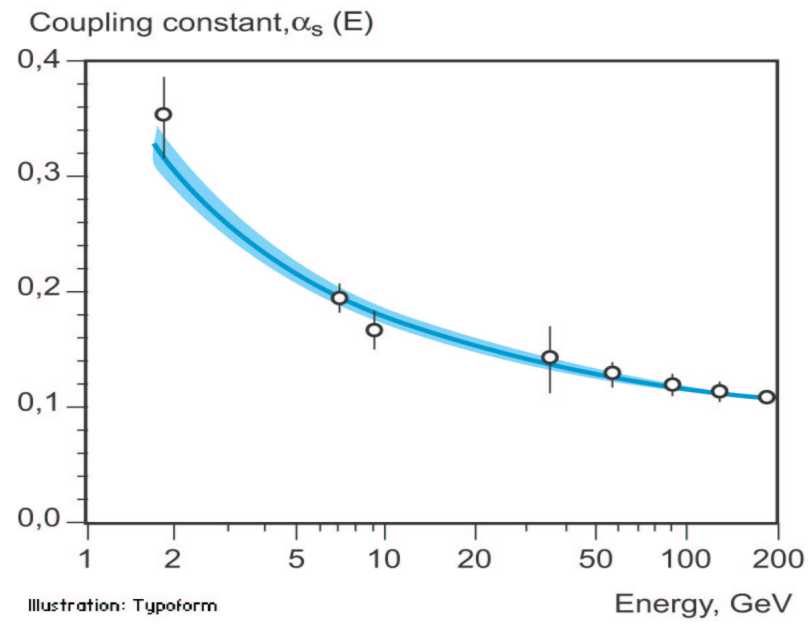

Figure 2.1: The value of the running coupling constant, $\alpha_{S}$, as a function of the energy scale $\mathrm{E}$. 
This characteristic running of $\alpha_{S}$ is used to explain the observed behavior of the strong interaction:

- Asymptotic freedom: At high energies (small distance) the strong interaction proceeds via colour field of reduced strength and the quarks and gluons behave as essentially free, noninteracting particles.

- Confinement: At low energies (or large distance) the strength of the colour field is increasing, since the potential behaves as $V(r) \sim \lambda r$, and in this way the quarks and gluons can never be observed as free particles. If two interacting partons are separated, the energy of the field increases so much that it creates new interacting particles and at the end it is left with colourless hadrons containing the partons. Therefore partons are not observed as free particles.

It is important to note that the asymptotic freedom property allows the application of perturbation theory to calculate cross section measurements in scattering processes where quarks and gluons are involved. Moreover, this property explains the partial success of the naive Quark Parton Model approach, which is going to be presented below.

\subsection{Deep Inelastic scattering and the Quark Parton Model}

Deep inelastic scattering (DIS) experiments have been important to develop QCD theory. The first series of this kind of experiments allowed to study the internal structure of hadrons and established the physical reality of quarks in the context of the Quark Parton Model, the predecessor of QCD. When the transfered momentum is much larger of $1 \mathrm{GeV}^{2}$, the process is in the DIS regime. In this case, the target loses its identity completely and the resulting final states are complicated multiparticle states whose study allows to gain insight into the internal structure of the initial target. Consider the scattering of a high energy charged lepton off a hadron target. Figure 2.2 illustrates the kinematics of a deep inelastic scattering $e P \rightarrow e X$, where $\mathrm{X}$ means any hadronic final state.

\footnotetext{
${ }^{2}$ If the particle momentum passed $1 \mathrm{GeV} / \mathrm{c}$, according to de Broglie's relation $\lambda=h / p$ structures smaller than $1 \mathrm{fm}$ (size of the proton) can be resolved
} 


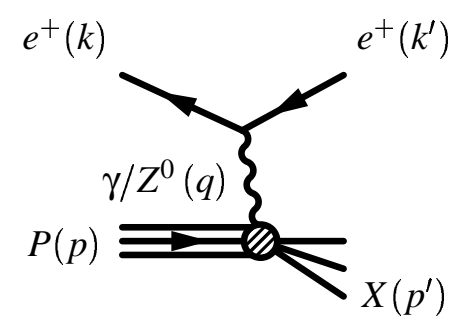

Figure 2.2: Schematic view of a deep inelastic scattering reaction: the photon $\left(\mathrm{o} Z^{0}\right)$ interacts with a quark inside the proton.

At a given center-of-mass energy, $\sqrt{s}$, the kinematics of a DIS event are completely described by two variables:

$$
Q^{2}=-q^{2}=\left(k-k^{\prime}\right)^{2} \quad \text { and } \quad x=\frac{Q^{2}}{2(p \cdot q)},
$$

where $k$ and $k^{\prime}$ are the 4-momenta of the ingoing and outgoing electron, respectively, and $p$ is the 4-momentum of the incoming proton. The variable $x$ is known as the Bjorken variable and can be interpreted as the fraction of the proton four-momentum carried by the struck quark. Figure 2.3 shows the kinematic range of DIS events accessible by different experiments, included the one probed by the Tevatron.

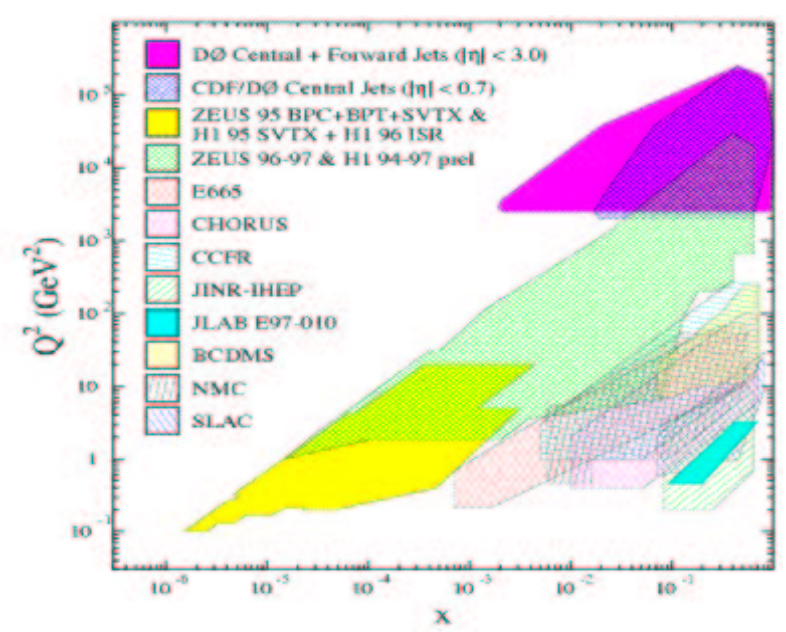

Figure 2.3: Kinematic range accessible by the Tevatron in Run I and various fixed target deep inelastic scattering experiments. 
Using these variables, the DIS cross section for the process $e P \rightarrow e X$ can be written as:

$$
\frac{d^{2} \sigma\left(e^{ \pm} p\right)}{d x d Q^{2}}=\frac{4 \pi \alpha^{2}}{x Q^{4}}\left(y^{2} x \cdot F_{1}\left(x, Q^{2}\right)+(1-y) \cdot F_{2}\left(x, Q^{2}\right) \mp y(1-y) x \cdot F_{3}\left(x, Q^{2}\right)\right)
$$

where $y$ is the ratio $Q^{2} / s x$ and the $\left\{F_{i}\right\}$ are the so-called proton structure functions. In order to give a physical interpretation of these structure functions, one can make use of the Quark Parton Model.

In the Quark Parton Model, which delivered the first description of the structure of protons and other hadrons, the proton consists of three free point-like spin 1/2 particles, called the valence quarks. One of the basic concepts of this model is that the $e P$ interaction can be viewed as incoherent scattering of electrons off partons inside the proton. This assumption can be justified by the following argument. The interaction time $t$ of the probe with the constituents of the proton is inversely proportional to its virtuality $Q^{2}(t \propto 1 / Q)$. Thus at large enough $Q$ the interaction time of the scattering process is much smaller than the lifetime of the fluctuations inside the proton. Therefore, the probe sees free, i.e. non-self-interacting, constituents in the proton, also called partons. Thus, the $e P$ cross section can be formulated as an incoherent sum of elastic electron parton scattering. Introducing the parton densities $f_{i}(x)$, also called parton distribution functions (PDFs), one obtains:

$$
F_{1}(x)=\frac{1}{2} \sum_{i} e_{i}^{2} f_{i}(x) \quad \text { and } \quad F_{2}(x)=\sum_{i} e_{i}^{2} x f_{i}(x)
$$

The term $f_{i}(x) d x$ gives the probability of finding a parton of type $i$ in the proton carrying a fraction between $x$ and $x+d x$ of the proton momentum and $e_{i}$ is the charge of the parton in units of electron charge. In 1969, Bjorken argued that the structure functions at large $Q^{2}$ and finite $x$ are functions of $x$ alone. This behavior, represented by

$$
F_{i}\left(x, Q^{2}\right) \rightarrow F_{i}(x)
$$

is known as scale invariance or Bjorken scaling [3]. This behavior can be easily explained inside the Quark Parton Model. If the particles within the proton are point-like, one would expect its structure to be independent of the resolution power of the probe and thus $Q^{2}$. This leads to the fact that the parton densities and structure functions only depend on the variable $x$ and not on 
$Q^{2}$. The Bjorken scaling was showed very clear by experimental results on $F_{2}$ measurements from SLAC [4], confirming at first the Quark Parton Model. It should be mentioned that the fractional charge of the partons and the number of three valence quarks in the proton were also experimentally confirmed, in this last case using neutrino-nucleon scattering [5].

If the proton were solely to be constituted of charge quarks, the integration of the parton densities over all partons inside the proton and over all the kinematic range of $x$ should be equal to unity:

$$
\int_{0}^{1} d x \times \sum_{i} f_{i}(x)=1
$$

but the experimental value turned out to be 0.5 [6]. The conclusion was that quarks carried half of nucleon momentum and the remainder one must be carried by partons that do not feel the electroweak force, namely gluons. This was the first gluons evidence, leading to the replacement of the Quark Parton Model by the QCD theory.

The appearance of gluons brought about a fundamental change of the dynamics inside the proton. The quarks inside the proton radiate gluons, and these gluons can then radiate more gluons or split into quark-antiquark pair, $q \bar{q}$, which themselves radiate gluons with smaller momentum fraction, and so on. The radiation of gluons results in a violation of the scaling behavior with a logarithmic dependence on $Q^{2}$ which is also experimentally observed. The violation of Bjorken scaling is naturally described by this example: a photon interacting with a quark at a certain $Q_{0}^{2}$ probes the proton with a finite resolution proportional to $1 / Q_{0}^{2}$. If the photon probes the same quark at a higher $Q^{2}$, the quark might have radiated a gluon not visible at $Q_{0}^{2}$ and the photon effectively interacts with a quark carrying less momentum. The result is a dependence of the structure functions on $Q^{2}$. In addition, the gluon appearance promoted a change in the structure of the proton to be three valences quarks (uud), which carry its electric charge and baryon quantum numbers, gluons and a sea of light $q \bar{q}$ pairs. An example of the parton distribution functions in the proton is illustrated in figure 2.4, determined at $Q=2 \mathrm{GeV}$. Notice that the valence quarks dominate a large $\mathrm{x}$ and gluon dominates a low $\mathrm{x}$.

Perturbative QCD (pQCD) does not predict the form of the PDFs but it is possible to obtain predictions about the variation with the scale. The processes that generate the parton interactions, to first order in $\alpha_{s}$, are gluon radiation $(q \rightarrow q g)$, gluon splitting $(g \rightarrow g g)$ and quark pair production $(g \rightarrow q \bar{q})$. The effect of all interactions is described by so called splitting functions: $P_{q q}(x / z)$, $P_{g q}(x / z), P_{g g}(x / z)$ and $P_{q g}(x / z)$. Figure 2.5 illustrates these functions at leading order. 


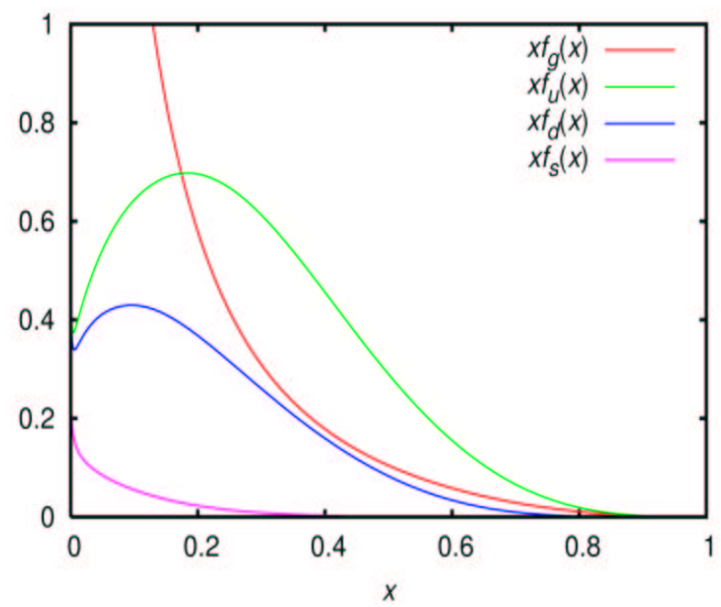

Figure 2.4: Example of parton distribution functions at $\mathrm{Q}=2 \mathrm{GeV}$ for gluons (red), up (green), down (blue), and strange (violet) quarks.
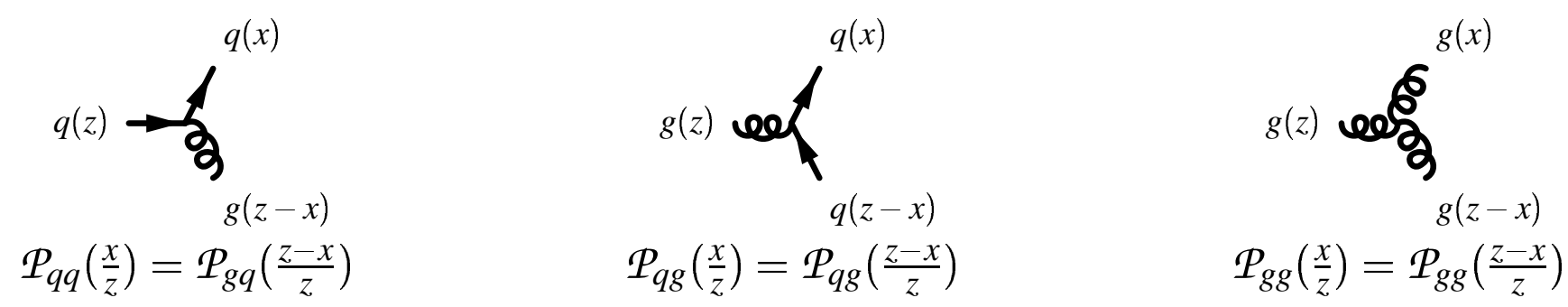

Figure 2.5: The processes related to the lowest order QCD splitting functions. Each splitting function $P_{p^{\prime} p}(x / z)$ gives the probability that a parton of type $p$ convert into a parton of type $p^{\prime}$, carrying fraction $\mathrm{x} / \mathrm{z}$ of the momentum of parton $p$. 
Each function $P_{p^{\prime}}(x / z)$ represents the probability that a parton of type $p$ radiates a quark or gluon and becomes a parton of type $p^{\prime}$ carrying fraction $\mathrm{x} / \mathrm{z}$ of the momentum of parton $p$. The splitting functions have been calculated using perturbative QCD and the expressions associated to these diagrams are:

$$
\begin{aligned}
P_{q q}(z) & =\frac{4}{3} \frac{1+z^{2}}{1-z}, \\
P_{q g}(z) & =\frac{1}{2}\left[z^{2}+(1-z)^{2}\right], \\
P_{g q}(z) & =\frac{4}{3} \frac{1+(1-z)^{2}}{z}, \\
P_{g g}(z) & =6\left[\frac{z}{1-z}+\frac{1-z}{z}+z(1-z)\right] .
\end{aligned}
$$

The evolution of the parton densities in $Q^{2}$ can be written in terms of these splitting functions:

$$
\begin{aligned}
& \frac{d q_{i}\left(x, Q^{2}\right)}{d \log \left(Q^{2}\right)}=\frac{\alpha_{s}}{2 \pi} \int_{x}^{1}\left(q_{i}\left(z, Q^{2}\right) P_{q q}\left(\frac{x}{z}\right)+g\left(z, Q^{2}\right) P_{q g}\left(\frac{x}{z}\right)\right) \frac{d z}{z} \\
& \frac{d g\left(x, Q^{2}\right)}{d \log \left(Q^{2}\right)}=\frac{\alpha_{s}}{2 \pi} \int_{x}^{1}\left(\sum_{i} q_{i}\left(z, Q^{2}\right) P_{g q}\left(\frac{x}{z}\right)+g\left(z, Q^{2}\right) P_{g g}\left(\frac{x}{z}\right)\right) \frac{d z}{z}
\end{aligned}
$$

where, the first equation describes the change of the quark densities with $Q^{2}$ due to gluon radiation and gluon splitting and, the second equation describes the change of the gluon density with $Q^{2}$ due to gluon radiation off quarks and gluons. These equations are called the DGLAP (Dokshitzer, Grobov, Livatov, Altarelli and Paris) equations [7, 8]. The equations assume massless partons and are hence only valid for gluons and the light quarks ( $\mathrm{u}, \mathrm{d}$ and $\mathrm{s}$ ). The DGLAP evolution are formally derived in the leading logarithm approximation (LLA), where terms of the form $\alpha_{s}^{n} \cdot\left(\ln Q^{2}\right)^{n}$, which give the dominant contribution at large $Q^{2}$ and large $x$, are summed to all orders. In a field theory having asymptotic freedom such approximation proves to be asymptotically exact. Experiments can measure the parton densities at a given scale $Q_{0}^{2}$ and the DGLAP equations can give predictions for the values for each parton density at other scales $Q^{2}$. As example, figure 2.6 illustrates the structure function $F_{2}$ in deep inelastic scattering from ZEUS and fixed targets. Farther discussion about PDFs is given in section 2.5. 


\section{ZEUS}

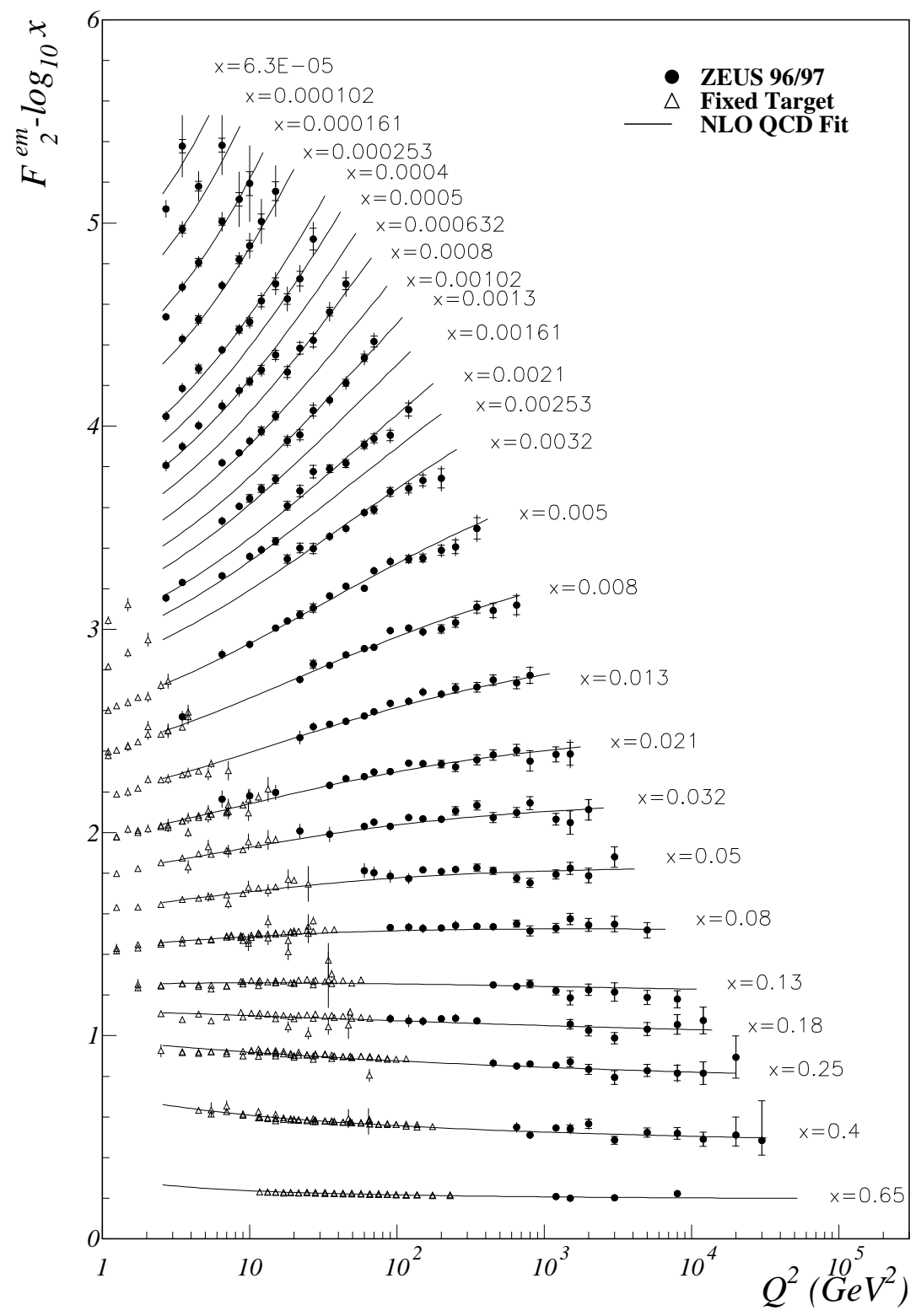

Figure 2.6: Data on the structure function $F_{2}$ in DIS from ZEUS and fixed target experiments. The curves are QCD fits. 


\subsection{Hadron-hadron processes and QCD Factorization}

An essential ingredient in the description of the DIS above is the concept of QCD Factorization, that allows to extract universal PDFs that can be employed in other hadron processes. The QCD Factorization theorem stays that a given success can be factorized into short- and long-distance related parts, where the long-distance physics is included in the PDFs.

The cross section for a hard scattering process initiated by two hadrons with four-momenta $P_{1}$ and $P_{2}$ can be written as:

$$
\sigma\left(P_{1}, P_{2}\right)=\sum_{i, j} \int d x_{1} d x_{2} f_{i}\left(x_{1}, \mu_{F}^{2}\right) f_{j}\left(x_{2}, \mu_{F}^{2}\right) \hat{\sigma}_{i j}\left(p_{1}, p_{2}, \alpha_{s}\left(\mu_{F}^{2}\right), Q^{2} / \mu_{F}^{2}\right)
$$

where the momenta of the partons which participate in the hard interaction are $p_{1}=x_{1} P_{1}$ and $p_{2}=x_{2} P_{2}$. The $\hat{\sigma}_{i j}$ is the parton-parton cross section and $f_{i}\left(x_{1}, \mu_{F}^{2}\right)$ are the well-known PDFs defined at a factorization scale, $\mu_{F}$. This factorization scale $\mu_{F}$ is an arbitrary parameter. It can be thought of as the scale which separates the soft and the hard processes. Thus a parton emitted with small transverse momentum less than the scale $\mu_{F}$ is considered part of the hadron structure and is absorbed into the parton distribution. Processes with transverse momentum larger than $\mu_{F}$ is part of the parton-parton cross section. The scale $\mu_{F}$ is typically chosen to be of the order of the hard scale Q, which characterizes the parton-parton interaction. Principally, any observable should be invariant under variations of this scale. This is formally expressed as:

$$
\mu^{2} \frac{d}{d \mu^{2}} \Gamma=0
$$

where $\Gamma$ is the observable we are interested in. In the perturbative approach, this equation has to be applied to the perturbative expansion of the observable,

$$
\Gamma=\Gamma_{0}+\alpha_{s} \Gamma_{1}+\alpha_{s}^{2} \Gamma_{2}+\ldots
$$

therefore, the equation transforms into

$$
\mu^{2} \frac{d}{d \mu^{2}} \sum_{j=0}^{N} \alpha_{s}^{j} \Gamma_{j}=O\left(\alpha_{s}^{N+1}\right),
$$

showing that the variation of the observable with the scale is given by terms which were not included in the perturbative expansion. The more terms included in the perturbative expansion, the weaker the dependence on $\mu$ will be. 
Similar to QED, different types of divergences appear in the pQCD calculations. The renormalization is the standard regularization procedure used to solve these divergences [9]. The procedure is not unique, i.e. there is a renormalization scheme selection, which is chosen depending on the properties of the parameters needed in the calculation. As in the factorization procedure, the renormalization introduces a scale, $\mu_{R}$, at which the renormalization is performed and any physical observable should be invariant under variations of this scale. However, a theoretical dependence is obtained in $\mathrm{pQCD}$ since the perturbative expansion is performed only to a given order.

\subsection{Parton Distribution Functions}

The partonic structure of hadrons plays a fundamental role in elementary particle physics. The comparison of data with SM predictions, precision measurements of SM parameters, and searches for signals of physics beyond the SM, all rely on the parton picture of hadronic beam particles.

As already mentioned, $\mathrm{pQCD}$ is not able to predict the $\mathrm{x}$-dependence of the PDFs. PDFs at a given scale $Q_{0}^{2}$ are extracted from fits to data and DGLAP equations are used to predict PDFs to a higher

scale $Q^{2}$. The PDFs are parametrized and the parameters are determined by a $\chi^{2}$ minimization over data from different type of measurements: structure functions in deep-inelastic $e, \mu$ or $v$ scattering, measurements of Drell-Yan production, $\mathrm{W}$-asymmetry in $p \bar{p}$ collisions and inclusive jet cross sections. Different groups provide parameterizations of parton densities. Among others, PDFs come from Martin, Roberts, Stirling and Thorne (MRST) group [10] and the "Coordinated Theoretical-Experimental Project on QCD”( CTEQ Collaboration) [11].

\subsubsection{MRST and CTEQ parametrizations}

The MRST group uses the parametrization for quarks and gluons at a scale $Q_{0}^{2}$ :

$$
x f_{i}\left(x, Q_{0}^{2}\right)=A_{i} \cdot x^{\delta_{i}}(1-x)^{\eta_{i}}\left(1+\varepsilon_{i} \sqrt{x}+\gamma_{i} x\right),
$$

where $f_{i}$ is a particular parton density and $A_{i}, \delta_{i}, \eta_{i}, \gamma_{i}$ are the parameters to be fitted. The $Q^{2}$ evolution is carried out using NLO DGLAP evolution equations. Not all the normalization factors $A_{i}$ are free parameters, but some are fixed from flavour or momentum sum rules. The charm content of the sea quarks is generated by gluon splitting as governed by the DGLAP equations. 
The CTEQ group takes a very similar approach. For quark distributions the same functional form as in equation 2.18 is chosen, whereas the gluon density is parameterized as:

$$
x g\left(x, Q_{0}^{2}\right)=A_{0} \cdot x^{A_{1}}(1-x)^{A_{2}}\left(1+A_{3} x^{A_{4}}\right) .
$$

In 1996, measurements of the inclusive jet cross section based on a CDF Run IA data sample of $19.5 p b^{-1}$ [12] showed a significant excess of the data over the available theoretical predictions at high $E_{T}$, see figure 2.7. A careful re-evaluation of uncertainties on PDFs indicated that the apparent excess could be accommodated by a modified gluon distribution at high-x, and motived the derivation from both collaborations of new PDFs, which specially gave higher weight to the high $E_{T}$ CDF data points.

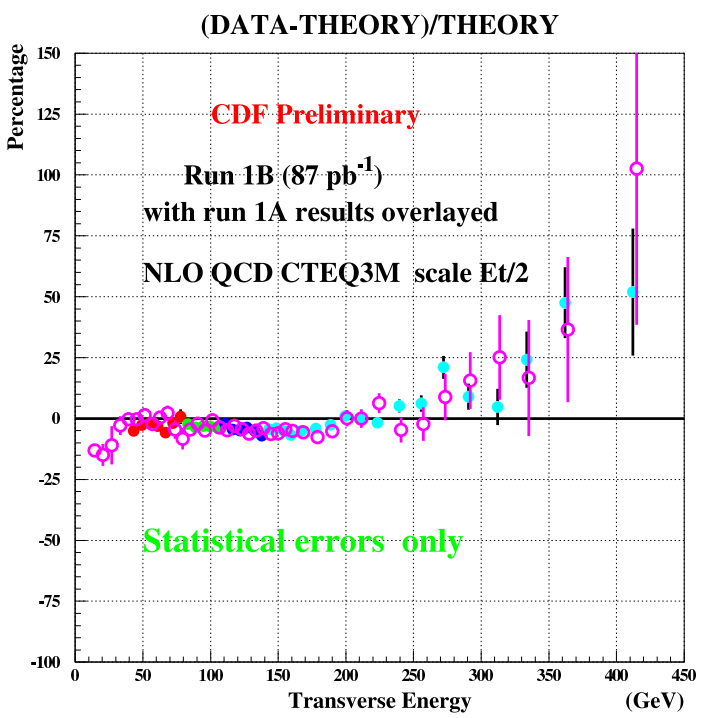

Figure 2.7: CDF Run I Cross section results compared to NLO calculations, using CTEQ3M PDFs set. The data are significantly higher than NLO QCD prediction for $E_{T}>250 \mathrm{GeV}$. 
CTEQ Collaboration presented the set of distributions known as CTEQ4 [13], where the gluon contribution was increased at high-x. The NLO predictions using the resulting fit described CDF data in all the $E_{T}$ range, as it is shown in figure 2.8. Since then, new parametrization have been generated. The most recent set from CTEQ collaboration is the CTEQ6 [14]. In this case, the parameterization for quarks and gluons is:

$$
x f\left(x, Q_{0}^{2}\right)=A_{0} \cdot x^{A_{1}}(1-x)^{A_{2}} e^{A_{3}} x\left(1+e^{A_{4}} x\right)^{A_{5}},
$$

with independent parameters for parton flavour combination $u_{v} \equiv u-\bar{u}, d_{v} \equiv d-\bar{d}$ and gluons.

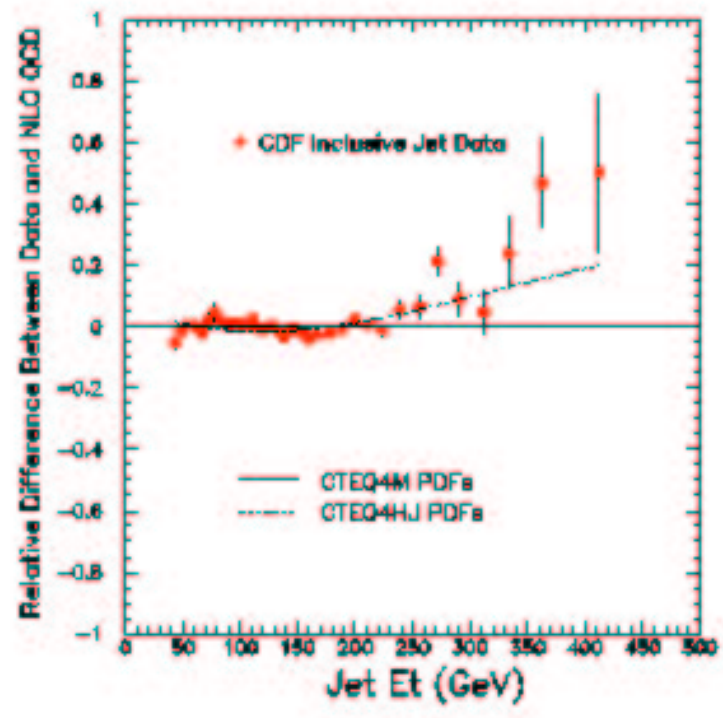

Figure 2.8: A comparison of the CDF Run I cross section to NLO calculations using CTEQ4M and CTEQ4HJ PDFs sets. At high $E_{T}$ the data are well described by CTEQ4HJ, in which the gluon contribution at high-x was modified. 
The set called CTEQ6.1M [15] is the one used in this thesis for the nominal NLO calculations. The dominant difference between the CTEQ6M and its predecessor, CTEQ5M sets, is the increased gluon distribution in CTEQ6M at large values of $\mathrm{x}$, while the quark distributions are nearly unchanged. This translates into an increase in the fractional contributions of the quarkgluon and gluon-gluon subprocesses in $p \bar{p}$ collisions.

In the final results, the parametrization of the parton densities coming from the MRST group is used too. One of the most recent set is denoted by MRST2004 [16], where the parameterization for gluon is extended to:

$$
x g\left(x, Q_{0}^{2}\right)=A_{g} \cdot x^{\delta_{g}}(1-x)^{\eta_{g}}\left(1+\varepsilon_{g} \sqrt{x}+\gamma_{g} x\right)-A_{-} \cdot x^{\delta_{-}}(1-x)^{\eta_{-}} .
$$

\subsubsection{PDFs uncertainties}

A Hessian method is used to evaluate the PDFs uncertainties. A brief description of the method is given below, for more details see [17].

In the Hessian method, a large matrix $(20 \times 20$ for CTEQ, $15 \times 15$ for MRST), with dimensions equal to the number of free parameters in the fit, has to be diagonalized. The result is 20 (15) orthogonal eigenvectors for CTEQ (MRST), denoted as $a_{i}$, which provides the basis for the determination of the PDFs uncertainties for any cross section. The Hessian matrix can be expressed as:

$$
H_{i j}=\frac{1}{2} \frac{\partial^{2} \hat{\chi}^{2}}{\partial a_{i} \partial a_{j}} .
$$

This matrix determines the behavior of $\hat{\chi}^{2}(a)$ in the neighborhood of the minimum. The point $a_{0}$ in the $n$-dimensional parameter space, where $\hat{\chi}^{2}(a)$ is minimum, is the best fit to the global data set. Points in some small neighborhood of $a_{0}$ are also acceptable fits. For each eigenvector two displacements from $a_{0}$, in the + and - directions along the vector, denoted $a_{i}^{+}$and $a_{i}^{-}$for the $i^{t h}$ eigenvector are considered. At these points, $\hat{\chi}^{2}=\hat{\chi}_{0}^{2}+T^{2}$ where $\hat{\chi}_{0}^{2}=\hat{\chi}^{2}\left(a_{0}\right)=$ the minimum, and $T$ is a parameter called tolerance. Any PDFs set with $\hat{\chi}^{2}-\hat{\chi}_{0}^{2}<T^{2}$ is considered to be an acceptable fit to the global data set. In particular, the $2 n$ PDFs sets $a_{i}^{ \pm}$span the parameter space in the neighborhood of the minimum. CTEQ group choses $T^{2} \sim 100$ and MRST group uses $T^{2} \sim 50$.

Any quantity $\Gamma$ that depends on PDFs has a predicted value $\Gamma_{0}=\Gamma\left(a_{0}\right)$ and an associated, a priori asymmetric, uncertainty $\delta \Gamma$. The $+(-)$ uncertainties are calculated as: 


$$
\delta \Gamma_{+}=\left(\sum_{k=1}^{n}\left[\max \left(\Gamma\left(a_{i}^{+}\right), \Gamma\left(a_{i}^{-}\right), \Gamma\left(a_{0}\right)\right)-\Gamma\left(a_{0}\right)\right]^{2}\right)^{1 / 2}
$$

and

$$
\delta \Gamma_{-}=\left(\sum_{k=1}^{n}\left[\min \left(\Gamma\left(a_{i}^{+}\right), \Gamma\left(a_{i}^{-}\right), \Gamma\left(a_{0}\right)\right)-\Gamma\left(a_{0}\right)\right]^{2}\right)^{1 / 2}
$$

In figure 2.9 the uncertainties on gluon and u-quark distributions are shown. The u-quark distribution is tightly constrained for $\mathrm{x} \leq 0.8$, whereas the uncertainty on the gluon distribution can be larger than a factor of 2 at high $\mathrm{x}$.
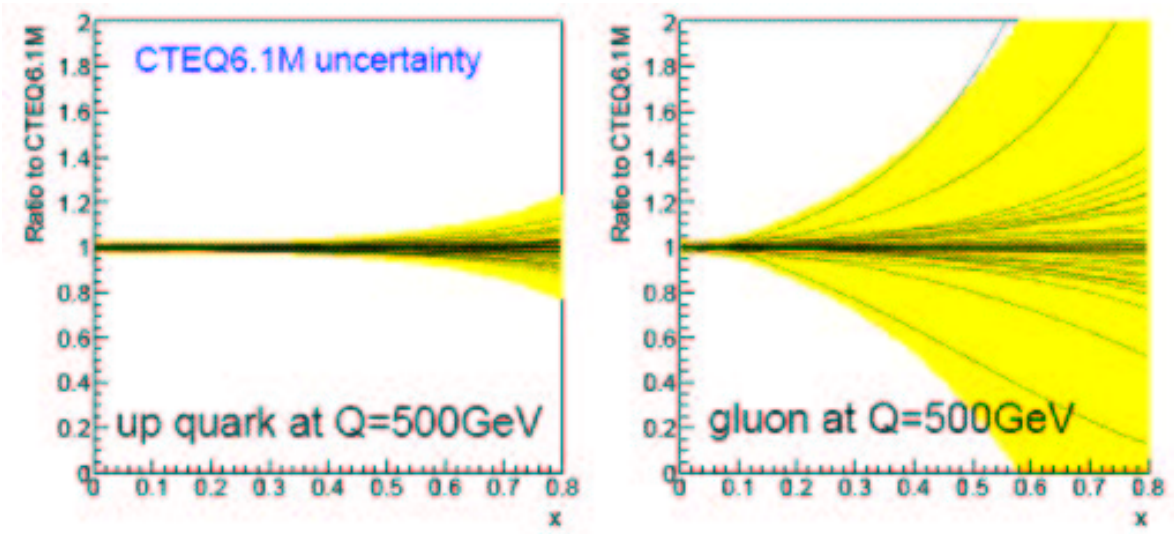

Figure 2.9: Uncertainty on gluon and u-quark PDFs. The yellow bands represent the global uncertainty. The curves are the ratios of the 40 eigenvector basis sets to the standard set, CTEQ6.1M.

\section{6 pQCD calculation for jet production}

The leading order calculations for the jet production include $2 \rightarrow 2$ parton scattering. The diagrams that contribute to are shown in figure 2.10. As it is illustrated in figure 2.11, the low $E_{T}$ cross section is dominated by qg and gg scattering, whereas at high $E_{T}$ the important subprocesses are qg and qq scattering. 


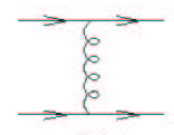

(a)
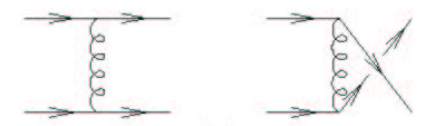

(b)
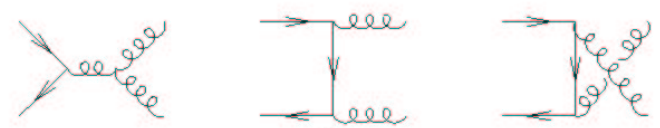

(c)
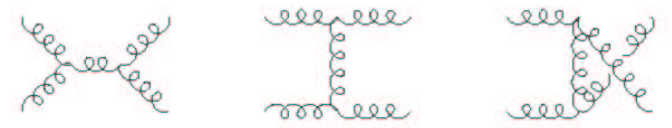

(d)

Figure 2.10: Diagrams for jet production at LO in a hadron collider. All the other lowest order jet production diagrams are related to this set by crossing.
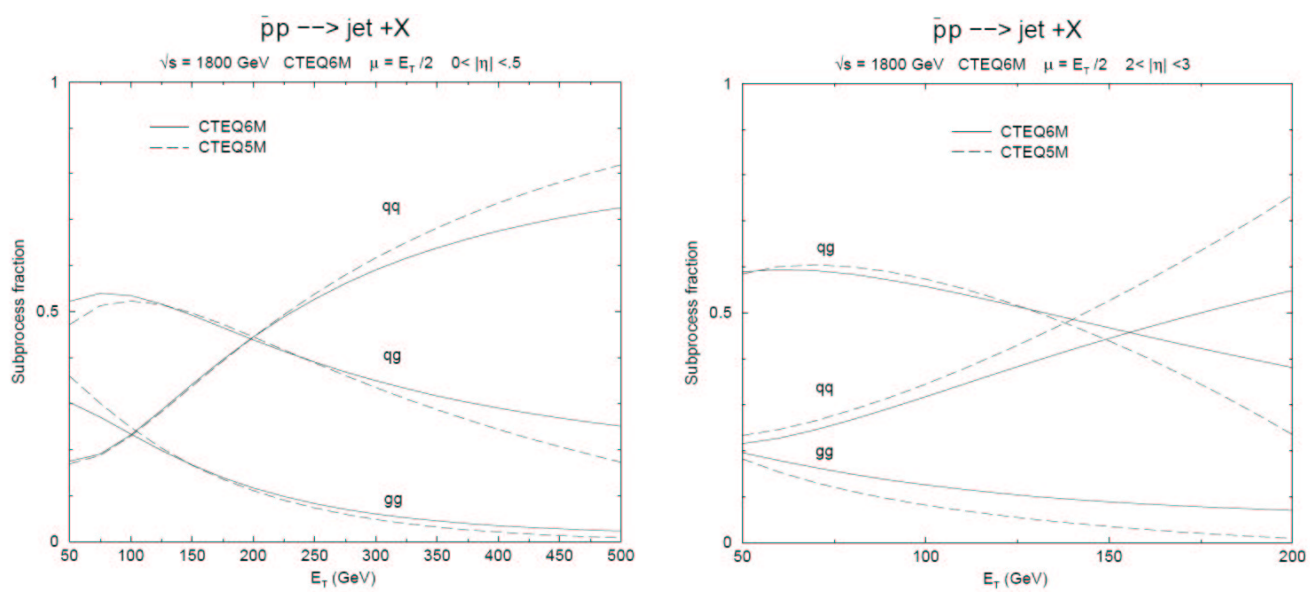

Figure 2.11: The subprocesses gg,gq and qq contributions to jet production in the central (left) and forward (right) rapidity regions at $\sqrt{s}=1.8 \mathrm{TeV}$ calculated by CTEQ collaboration. Similar contributions are expected at $1.96 \mathrm{TeV}$. 
Mathematically, the inclusive jet cross section at parton level can be described [1] by matrix element $\mathrm{M}$ as:

$$
\frac{1}{2 \pi p_{T}} \frac{d^{2} \hat{\sigma}}{d p_{T} d y}=\frac{1}{2 \hat{s}} \frac{1}{8 \pi^{2}} \bar{\sum}|M|^{2} \delta(\hat{s}+\hat{t}+\hat{u})
$$

where $\bar{\Sigma}$ denotes the average and sum over the initial and final state spins and colours respectively ${ }^{3}$. Expressions for the LO matrix elements squared $\bar{\Sigma}|M|^{2}$ are given in table 2.2, using the following notation: $\operatorname{parton}_{1}\left(p_{1}\right)+\operatorname{parton}_{2}\left(p_{2}\right) \rightarrow \operatorname{parton}_{3}\left(p_{3}\right)+\operatorname{parton}_{4}\left(p_{4}\right), \hat{s}=\left(p_{1}+p_{2}\right)^{2}$, $\hat{t}=\left(p_{1}-p_{3}\right)^{2}$ and $\hat{u}=\left(p_{2}-p_{3}\right)^{2}$. To give an idea of the relative importance of each process the value of the $\bar{\Sigma}|M|^{2}$ at $90^{\circ}$ in the center of mass $\left(\theta^{*}=\pi / 2, \hat{t}=\hat{u}=-\hat{s} / 2\right)$ is shown.

Nowadays, the jet cross section calculation are done a next-to-leading order (NLO) $\mathrm{O}\left(\alpha_{S}^{3}\right)$. The NLO calculations include one-loop corrections to the LO process and real radiation corrections, becoming a $2 \rightarrow 3$ process. Details about this calculation can be found in [18]. Unfortunately, due to the number of diagrams involve in the next-to-next-to-leading order (NNLO) calculations, which include two-loop corrections to Born, one-loop corrections to single real radiation terms and double real radiation correction, the predictions at this level are not available yet.

\subsection{Beyond NLO: MonteCarlo programs}

As it was mentioned in previous section, complete perturbative calculations in QCD have been performed only to NLO in most of the cases. However, higher-order terms can not be neglected in the case of soft-gluon radiation and collinear configurations. The leading contributions of these soft and collinear topologies, and the corresponding enhanced virtual corrections, can be identified and summed to all orders, improving the convergence of the perturbative series. Parton shower is an approximated result in which such enhanced terms are taken into account to all orders. It is implemented in computer simulations, called QCD Monte Carlo generator programs (MC). The MC, not only include this parton shower, which allows partons to split into pairs of other partons, but also a phenomenological model to produce colorless hadrons from the resulting partons. The implementation and modeling of the parton shower and hadronization processes in two of the most used MC programs, PYTHIA [19] and HERWIG [20], are presented below.

\footnotetext{
${ }^{3}$ Experimentally is impossible to measure the total jet cross section in an experiment since jets are counted only in the fiducial volume of the detector. Thus experiments measure the differential cross section in jet transverse momentum, $p_{T}$, and jet rapidity, $y$, with $y=\frac{1}{2} \ln \frac{E+p_{z}}{E-p_{z}}$
} 


\begin{tabular}{|c|c|c|}
\hline process & $\overline{\bar{\Sigma}}|M|^{2} / g^{4}$ & $\theta^{*}=\pi / 2$ \\
\hline$q q^{\prime} \rightarrow q q^{\prime}$ & $\frac{4}{9} \frac{\hat{s}^{2}+\hat{u}^{2}}{\hat{t}^{2}}$ & 2.22 \\
\hline$q \overline{q^{\prime}} \rightarrow q \bar{q}^{\prime}$ & $\frac{4}{9} \frac{\hat{s}^{2}+\hat{u}^{2}}{\hat{t}^{2}}$ & 2.22 \\
\hline$q q \rightarrow q q$ & $\frac{4}{9}\left(\frac{\hat{s}^{2}+\hat{u}^{2}}{\hat{t}^{2}}+\frac{\hat{s}^{2}+\hat{t}^{2}}{\hat{u}^{2}}\right)-\frac{8}{27} \frac{\hat{S}^{2}}{\hat{u} \hat{t}}$ & 3.26 \\
\hline$q \bar{q} \rightarrow q^{\prime} \bar{q}^{\prime}$ & $\frac{4}{9} \frac{\hat{t}^{2}+\hat{u}^{2}}{\hat{s}^{2}}$ & 0.22 \\
\hline$q \bar{q} \rightarrow q \bar{q}$ & $\frac{4}{9}\left(\frac{\hat{s}^{2}+\hat{u}^{2}}{\hat{t}^{2}}+\frac{\hat{t}^{2}+\hat{u}^{2}}{\hat{s}^{2}}\right)-\frac{8}{27} \frac{\hat{u}^{2}}{\hat{s} \hat{t}}$ & 2.59 \\
\hline$q \bar{q} \rightarrow g g$ & $\frac{32}{27} \frac{\hat{t}^{2}+\hat{u}^{2}}{\hat{t} \hat{u}}-\frac{8}{3} \frac{\hat{t}^{2}+\hat{u}^{2}}{\hat{s}^{2}}$ & 1.04 \\
\hline$g g \rightarrow q \bar{q}$ & $\frac{1}{6} \frac{\hat{t}^{2}+\hat{u}^{2}}{\hat{t} \hat{u}}-\frac{3}{8} \frac{\hat{t}^{2}+\hat{u}^{2}}{\hat{s}^{2}}$ & 0.15 \\
\hline$g q \rightarrow g q$ & $-\frac{4}{9} \frac{\hat{s}^{2}+\hat{u}^{2}}{\hat{s} \hat{u}}+\frac{\hat{u}^{2}+\hat{s}^{2}}{\hat{t}^{2}}$ & 6.11 \\
\hline$g g \rightarrow g g$ & $\frac{9}{2}\left(3-\frac{\hat{u} \hat{u}}{\hat{s}^{2}}-\frac{\hat{s} \hat{u}}{\hat{t}^{2}}-\frac{\hat{s} \hat{t}}{\hat{u}^{2}}\right)$ & 30.4 \\
\hline
\end{tabular}

Table 2.2: LO jet production matrix elements squared $\bar{\Sigma}|M|^{2} / g^{4}$. The third column gives the sizes of the contribution from each process at $\theta^{*}=\pi / 2$.

\subsubsection{Parton shower}

The parton shower in the MC serves two main purposes. The first one is to provide an estimation of these higher-order corrections that are enhanced by large kinematic logarithms and second, to generate high-multiplicity partonic states which can then be converted into the observed hadrons by a hadronization mechanism.

Schematically, the parton shower is a random process. During showering, successive values of a scale $t$, a momentum fraction $z$ and an azimuthal angle $\phi$ are generated, together with the flavors of the partons emitted. The evolution is based on the Sudakov form factors [21], which expresses the probability that a parton does not branch between some initial maximum and minimum scale 
$t$. The branching processes: $q \rightarrow g q, g \rightarrow g g$ and $q \rightarrow q \bar{q}$ are described by the DGLAP equations introduced in section 2.3. Once a branching has occurred, say $i \rightarrow j k$ at scale $t_{i}$, the evolution of the daughter partons $j$ and $k$ has to be generated. At the simplest level, their evolution starts at scale $t_{i}$ and the next values $t_{j}$ and $t_{k}$ are obtained using the appropriate Sudakov form factors. Usually, $t$ is proportional to the virtuality $Q^{2}$, thus the virtualities of the daughters are constrained by the kinematic relation $\sqrt{t_{j}}+\sqrt{t_{k}}<\sqrt{t_{i}}$, where $t_{i}=E_{i}^{2}\left(1-\cos \theta_{i}\right)$ being $\theta_{i}$ the opening angle in the branching $i \rightarrow j k$, and where an angular ordering is imposed. This means that the opening angle $\theta_{j}$ of any subsequent branching of parton $j$ is less than $\theta_{i}$.

The final outcome of successive branchings is a parton shower in which each initial parton from the hard process is replaced by a jet of partons moving roughly in the same direction. The typical scale of relative transverse momenta between partons at the end of the shower is set by the cutoff $t_{0} \sim \Lambda_{Q C D}$, beyond that $\mathrm{pQCD}$ can not be applied. All these quarks, antiquarks, and gluons are not allowed to exist in isolation, as dictated by colour confinement. Thus, the next step in the MC programs is to group these coloured partons into the observed colourless hadrons using a phenomenological model referred to as hadronization.

\subsubsection{Hadronization}

Different models have been developed over the years to describe the hadronization phenomenon. Each model contains several parameters that are tuned using experimental data.

One of the parameter to be tuned with experiments is $t_{0}$. Ideally the hadronization should be independent of $t_{0}$. However, if the parameter increases, the shower finalizes before and there are less partons to be hadronizated. Thus the hadronization model should ideally has a parameter $t_{0}$ whose effect cancels when the parton shower and hadronization phases of jet fragmentation are combined. In practice, cancellation will be imperfect owing to deficiencies of the model.

One general approach to hadronization, based on the observation that perturbation theory seems to work well down to rather low scales, is the hypothesis of local parton-hadron duality, where one supposes only that the flow of momentum and quantum numbers at the hadron level tends to follow the flow established at the parton level. Hence, the flavour of the quark initiating a jet should be found in a hadron near the jet axis. 


\section{String model}

The assumption of linear confinement provides the starting point for the string model $[22,23]$. As the $q$ and $\bar{q}$ partons move apart from their common production vertex, the physical picture is that of a colour flux tube being stretched between the $q$ and the $\bar{q}$. If the tube is assumed to be uniform along its length, this automatically leads to a confinement picture with a linearly rising potential. As the $q$ and $\bar{q}$ move apart, the potential energy stored in the string increases, and the string may break by the production of a new $q^{\prime} \bar{q}^{\prime}$ pair, so that the system splits into two colour-singlet systems $q \bar{q}^{\prime}$ and $q^{\prime} \bar{q}$. If the invariant mass of either of these string pieces is large enough, further breaks may occur. In the string model, the string break-up process is assumed to proceed until only on-mass-shell hadrons remain, each hadron corresponding to a small piece of string with a quark in one end and an antiquark in the other. Charm and heavier quarks hence are not expected to be produced in the soft fragmentation, but only in perturbative parton-shower branchings $g \rightarrow q \bar{q}$. The baryons production is still a poorly understood area. In the simplest possible approach, a diquark in a colour antitriplet state is just treated like an ordinary antiquark, such that a string can break either by quark-antiquark or antidiquark-diquark pair production. If several partons are moving apart from a common origin, the details of the string drawing become more complicated. A schematic picture of the production of a multi-hadronic final state according to the string model is shown in figure 2.12 (left). Notice that whenever a gluon splits perturbatively into a quarkantiquark pair during the evolution of the parton shower, an additional string segmentation is produced. On the other hand, gluons which remain at the end of the shower lead to kinks in the string segment which connect them.

\section{Cluster model}

This model is quite different. The cluster hadronization model [24] is based on the colour preconfinement property of pQCD [25]. At the end of the parton shower evolution, the remaining gluons are split non-perturbatively into $q \bar{q}$ pairs. Neighbouring $q$ and $\bar{q}$ can be then combined into singlets cluster with a typical mass of a couple of $\mathrm{GeV}$. These clusters decay directly into two hadrons unless they are too heavy, when they decay to two clusters, or too light in which case the cluster decays to a single hadron. Experimentally it has been tested that this model describes quite well the hadronic energy and transverse momentum distribution for final states. Figure 2.12 (right) shows the cluster hadronization model. 


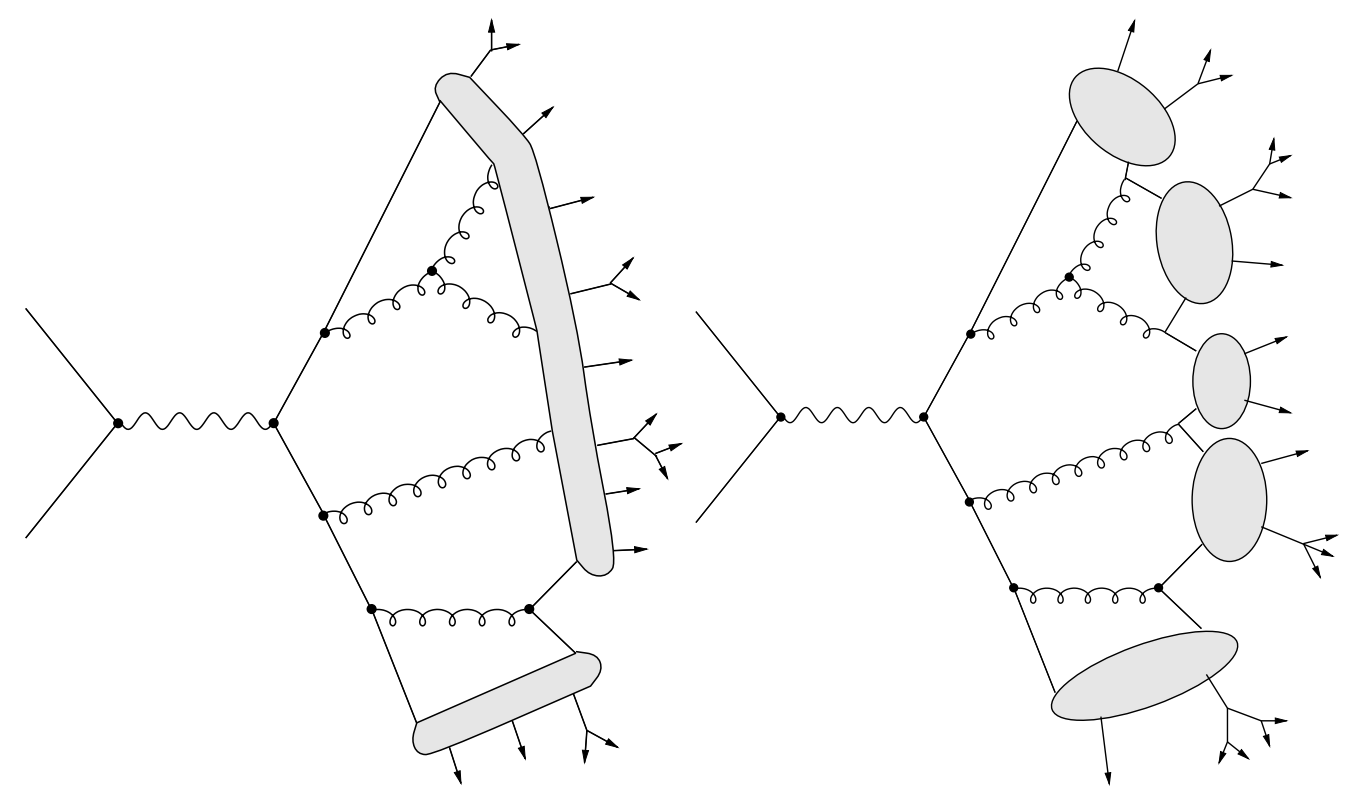

Figure 2.12: Parton shower with string (on the left) and cluster (on the right) hadronization models.

\subsection{Jet Phenomenology}

The result of the parton shower and hadronization processes is a collimated flow of particles called jets. To make quantitative comparison between theory and experiment, one must go beyond a qualitative definition and use a precise algorithm to define jets. A jet algorithm is not uniquely defined and the experimental results depends on the criteria used to reconstruct jets. Moreover, a well designed jet algorithm must have some basic properties: It should be well-defined and easy to calculate from the hadronic final state; It should be easy to calculate order-by-order in perturbation theory, this means, it should be infrared and collinear safe; It should have a close correspondence with the distribution of the final-state quarks and gluons that one is interested in. Although the basic hard scattering processes studied in different types of collisions can be described within the same theoretical framework, the overall event structure is quite different. For example, in $e^{+} e^{-}$annihilation into hadrons, the initial state is purely leptonic and the entire final state can be thought of an arising from the virtual boson which creates a $q \bar{q}$. Therefore, all the hadrons in the final state are associated with the hard scattering process. In contrast, in hadronlepton or hadron-hadron collisions, there are different contributions to the overall final state: only 
a fraction of the final-state hadrons is associated with the hard scattering process; there is a remainder form part from the beam remnants; and eventual soft, i.e. small transverse momentum, interactions of the remaining partons in the incident hadrons. Moreover, since only a fraction of the initial energy of the incoming hadron takes part in the hard process, the angular distribution of the final-state particles could be affected by the boost in the longitudinal direction. Therefore, the jet algorithms have to be modified accordingly the kinematic and dynamics of each type of collision. Over the years different algorithms have been proposed and used, some of them will be revised in this section.

\subsubsection{First jet algorithms}

The first evidence for quark jets was seen by the Mark I detector [26] at the SPEAR storage ring at SLAC, a $e^{+} e^{-}$collider at 3-7.4 GeV. The jet algorithm used was based on the definition of a jet axis. The axis was chosen to be the direction such that the sum of the squares of the momenta transverse to the axis, $p_{\perp}$, was a minimum. At each event was then assigned a value of "sphericity", which is defined as:

$$
S=\frac{3 \sum_{i} \vec{p}_{\perp}^{2}}{2 \sum_{i} \vec{p}_{i}^{2}}
$$

where the summation is over all the detected particles. S approaches to 0 for events with jets (bounded transverse momenta) and approaches to 1 for events with large multiplicity and isotropic phase-space particle distributions.

Approximately five years later, people from the TASSO collaboration at PETRA, a $e^{+} e^{-}$collider located at DESY that could reach more than $30 \mathrm{GeV}$, were concentrated on looking for the gluon. At that time, the gluon was one of the most interesting particles, theoretically expected but not yet experimentally observed. Since the gluon is the gauge particle for strong interactions, a way to produce a gluon was by the gluon bremsstrahlung process $e^{+} e^{-} \rightarrow q \bar{q} g$. Since the gluon, similar to the quark, is expected to hadronize into a jet, this process leads to a three-jet event. A new fast algorithm, also based on the determination of jet axis by minimizing the sum of the square momenta, was designed by San Lan Wu and Georg Zobernig to found a way to discover the gluon through these three-jet events [27]. Finally, it was in June 1979 when the TASSO Collaboration presented the first three-jet event, a $q \bar{q} g$ state, using the Zobernig and Wu's algorithm. At higher orders in perturbation theory, the final state can consist of more than three jets. It turned out that this algorithm was not well-suited to analyze these multijets events, and more generic jet algorithms started to be formulated. 
At present there are essentially two classes of algorithms in use: cone-type algorithms and clustertype algorithms. Generally, the cone-type algorithms define the jets using fixed geometrical structures which are positioned in the angular space occupied by the particles in such a way that the energy or the transverse energy is maximized. To specify an algorithm of this kind, the only requirements are the geometrical definition of the 'cone', usually is a circle in the angular space with a given radius, a momentum addition rule called the recombination scheme, and the criterion to be followed if two or more of these 'cones' overlap. In contrast, the cluster-type algorithms are characterized by successively finding pairs of particles that are 'nearby' in phase-space and merging them together, using also a recombination scheme, to form new 'pseudo-particles' which are then considered in the next iteration, instead of the two original ones. The cluster-type algorithms need a definition of distance to decide what 'nearby' particles means in a quantitative way. As an additional requirement, usually some scale is needed to stop the iterative procedure and to define the final jets from the pseudo-particles. Next, some of the cone-type algorithms and cluster-type algorithms are presented, starting from the original versions and followed by their specific implementations in the CDF experiment at Tevatron. It should be noted that in the discussion of jet algorithms, the word 'particles' is applied to any set of four-momenta. It could also refer to the partons in the theoretical calculation, or the energy deposits detected in calorimeter towers.

\subsubsection{Cone-based algorithms}

The cone-based algorithm is still the most popular at hadron colliders. The jets are characterized in term of variables that are invariant under boost along the beam axis. The variables are: transverse momenta, $p_{T}$, or the transverse energy, $E_{T}=E \sin \theta$; azimuthal angle around the beam direction, $\phi$; and pseudo-rapidity, $\eta=-\ln (\tan (\theta / 2)$. The algorithm forms jets by associating together particles whose trajectories lie within a circle of specific radius $R$ in $\eta \times \phi$ space.

\section{The UA1 and UA2 cone-based algorithms}

In the $80 \mathrm{~s}$, the UA1 and UA2 experiments were the firsts to use cone-based algorithms in a $p \bar{p}$ collider. The UA1 algorithm started ordering in decreasing transverse $E_{T}$ the cells of the calorimeter with $E_{T}>E_{T, \text { seed }}$. The value used for $E_{T, \text { seed }}$ was $2.5 \mathrm{GeV}$. The cell with the highest transverse

energy initiated the first jet. The next cell was added to the first if it was within a distance $R_{0}=1$. If the cell was outside this radius then a new jet was initiated. This procedure was repeated until all cells above the $E_{T, \text { seed }}$ threshold have been assigned to a jet. Finally, the cells with $E_{T}<E_{T, \text { seed }}$ 
are then added to each jet if $R<R_{0}$. In contrast UA2 used another approach. In the UA2 algorithm the cluster did not have a limited size in $\eta \times \phi$ space. Once all the calorimeter cells were ordered in decreasing $E_{T}$, starting for the highest one, all the neighboring cells were joined into the cluster if the $E_{T}$ exceeded a given threshold. In that case, the threshold was $0.4 \mathrm{GeV}$.

\section{The CDF cone-based algorithms: JetClu and MidPoint algorithms}

Some years later, the CDF experiment started to use also a cone-based algorithm, the JetClu algorithm. This begins defining a list of seeds, calorimeter towers with $E_{T}>1 \mathrm{GeV}$. Each seed is the starting geometric center for a cone. Thus, for a specific geometric center for the cone $\left(\phi_{C}, \eta_{C}\right)$ the 'particles' $i$ within the cone satisfy

$$
\Delta R_{i C} \equiv \sqrt{\left(\eta_{i}-\eta_{C}\right)^{2}+\left(\phi_{i}-\phi_{C}\right)^{2}} \leq R
$$

and the energy of the jet, using the snowmass convention [28], is defined as:

$$
E_{T}^{C}=\sum_{i \in \text { Cone }} E_{T}^{i}
$$

Then the jet geometric center, defined as

$$
\eta_{C}=\frac{\sum_{i \in \text { Cone }} E_{T}^{i} \eta^{i}}{E_{T}^{C}} \text { and } \phi_{C}=\frac{\sum_{i \in C o n e} E_{T}^{i} \phi^{i}}{E_{T}^{C}}
$$

is calculated. This new point in $\eta \times \phi$ is then used as the center for a new trial cone. As this calculation is iterated the cone center flows until a "stable" solution is found, i.e., until the centroid of the energy depositions within the cone is aligned with the geometric axis of the cone.

Unfortunately, nothing prevents the resulting final stable cones from overlap as it is shown in figure 2.13. Therefore, a procedure must be included in the cone algorithm to specify how to split or merge overlapping cones. These cones are merged if their shared transverse energy is larger than a fixed fraction (e.g., $\mathrm{f}=75 \%$ ) of the jet with smaller transverse energy; otherwise two jets are formed and the common towers are assigned to the jet closer in $\eta \times \phi$ space. 


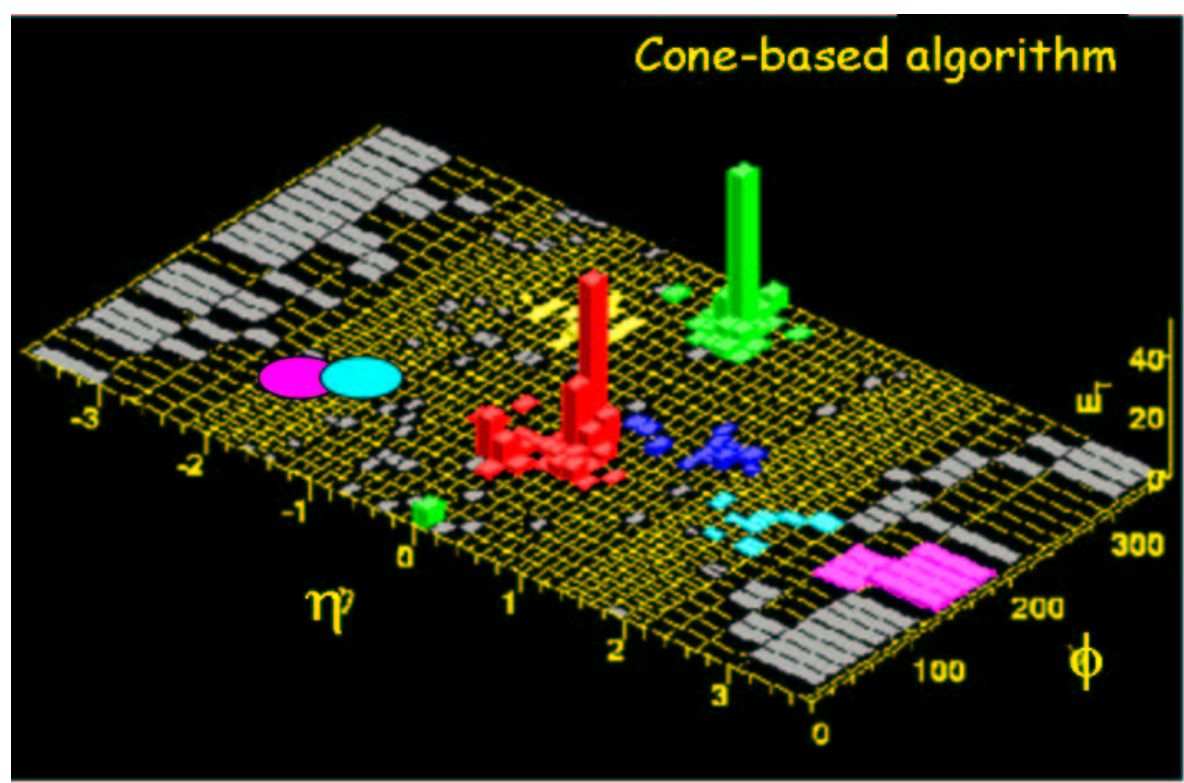

Figure 2.13: Lego plot of an event with reconstructed jets using a cone-based algorithm. This example illustrates an overlapping situation between two stable cones (red cluster).

After Run I, it was realized [29] that the iterative cone algorithm, as described above, is not infrared safe when applied to parton-level calculations. If two cones overlap in such a way that their centers can also be enclosed in one cone but there is little energy in the overlap region, then it turns out, as illustrated in figure 2.14, that the outcome is different depending on whether or not the overlap region contains a seed direction. This results in a logarithmic dependence on the seed cell threshold which would give a divergent cross section if the threshold was taken to zero for the purposes of making an idealized calculation. This divergence first shows up when there can be three nearby partons, which for jets in hadron collisions is NLO in the three-jet cross section and NNLO in the two-jet or inclusive one-jet cross sections. Moreover, this algorithm is sensitive to collinear radiation in the event, where the seed finding depends of calorimeter granularity. It is clear from figure 2.15 that the seed it is not considered anymore after its energy is split. In order to address these theoretical difficulties, an additional step, before the merging/splitting procedure, is included. The midpoint, in the $\eta \times \phi$ plane, between each pair of stable cones separated by less than $2 R$ is added to the list of jets. The clustering algorithm is again iterated until stability is achieved. This step gives the name to the jet algorithm: MidPoint algorithm [29]. 

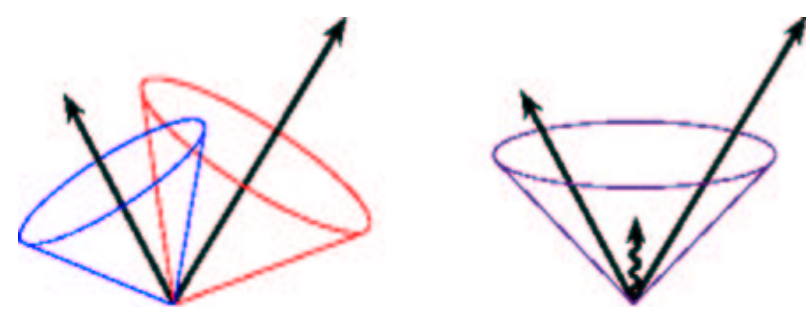

Figure 2.14: An illustration of infrared sensitivity in cone jet clustering. This example shows how the presence of a soft radiation between two jets may cause a merging of the jets that would not occur in the absence of the soft radiation.
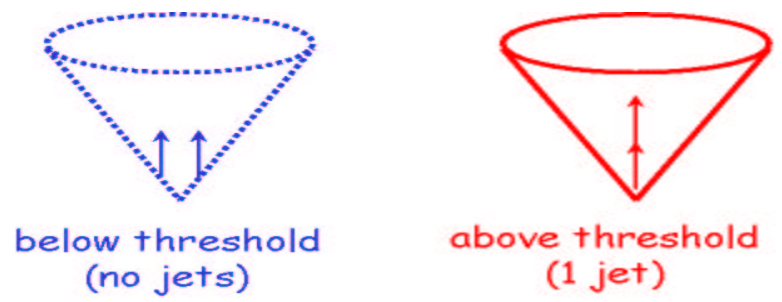

Figure 2.15: An illustration of collinear sensitivity in jet reconstruction.

However, the merging/splitting procedure applied to resolve situation with overlapping cones in data introduces an element of arbitrariness when the set algorithm is applied to theoretical calculations. Current NLO inclusive jet cross section calculations require the addition of an ad hoc parameter $R_{\text {sep }}$ [30]. The theoretical calculations use a cone algorithm with an enlarged cone size $R^{\prime}=R_{\text {sep }} \cdot R$, where $R_{\text {sep }}$ is typically 1.3 . In this way, theorists try to emulate effects of merging cones applied experimentally in the data, that results into jets with transverse size $(\eta \times \phi)$ larger than $R$. The use of different values for $R_{s e p}$ introduces an uncertainty in the theoretical prediction of the order of $5 \%$.

\subsubsection{The cluster-type algorithms}

According to QCD evolution and the development of proton showers as described in section 2.7, a sensible jet algorithm should cluster particles according to their relative transverse momentum instead of spacial separation. 


\section{The JADE algorithm}

The JADE algorithm [31], implemented by JADE collaboration at PETRA experiment, was the first cluster-type algorithm. It is very common in studies of jets in $e^{+} e^{-}$colliders in its original form or via some modifications. The jet reconstruction procedure starts by calculating the distance parameter $m_{i j}$ for all pairs of particles according to the expression

$$
m_{i j}^{2}=2 E_{i} E_{j}\left(1-\cos \theta_{i j}\right),
$$

where $E_{i}$ and $E_{j}$ are the energy of particle $i$ and $j$, and $\theta_{i j}$ is the angle between them. The variable $m_{i j}$ is the invariant mass of one particle decaying into $i$ and $j$ under the approximation that both particles are massless. The pair with the smallest $m_{i j}$ is combined into a single cluster by the addition of their four-momenta. The procedure is repeated until all remaining pairs have an invariant mass exceeding a preselected cut-off value, $m_{i j}^{2}>y_{\text {cut }} M^{2}$, determined by a resolution parameter $y_{c u t}$ and a reference mass $M$. The final clusters represent the jets of the event. However, with this distance parameter definition, the jet algorithm presents a problem when two soft gluons can be emitted by two leading back-to-back quarks. These two soft gluons could be combined with each other first, so that the final result is a "ghost jet" in a direction along which no original parton can be found. Such behaviours end up representing a serious challenge in perturbative calculations.

\section{The $k_{T}$-cluster algorithm}

The $k_{T}$ algorithm was initially introduced in $e^{+} e^{-}$interactions to solve the difficulties of JADE algorithm. It was based on the quantities

$$
k_{T,(i, j)}^{2}=2\left(1-\cos \theta_{i j}\right) \min \left\{E_{i}^{2}, E_{j}^{2}\right\},
$$

where $\theta_{i j}$ defines the distance between particles. For a given hard scale, $y_{c u t}$, the particles are clustered if $k_{T,(i, j)}<y_{c u t}$, and the algorithm iterates until all $k_{T,(i, j)}$ are larger than $y_{c u t}$. This algorithm also allows to vary $y_{c u t}$ event-by-event such that a fixed numer of jets is obtained and the resulting distribution is employed to study QCD radiation.

The $k_{T}$ algorithm can be extended to $e^{+} p$ and $p p$ interactions [32], for which an additional quantity $k_{T, i}$ is introduced to cluster particles around proton remnants. In DIS (lab frame) and $p p$ collisions, where the collision does not define the center of mass, transverse energies and rapidities are used instead of energies and polar angles. The $k_{T}$ algorithm has been successful used at LEP $\left(e^{+} e^{-}\right.$collider $)$and HERA ( $e^{ \pm} p$ collider) but it is relatively new at hadron colliders. 


\section{The CDF $k_{T}$ algorithm}

CDF Run II experiment is using the Ellis-Soper inclusive mode [33] implementation of the longitudinally-invariant $k_{T}$ algorithm. First, the variables

$$
k_{T, i}=p_{T, i}^{2} \quad \text { and } \quad k_{T,(i, j)}=\min \left(p_{T, i}^{2}, p_{T, j}^{2}\right) \cdot \Delta R_{i, j}^{2} / D^{2}
$$

are computed for each particle and pair of particles respectively, where $p_{T, i}$ denotes the transverse momentum of the $i$ particle, $\Delta R_{i, j}$ is the distance in $y \times \phi$ space, between each pair of particle, and $D$ is a parameter that approximately controls the size of the jet. All the $k_{T, i}$ and $k_{T,(i, j)}$ values are collected into a single sorted list. In this combined sorted list, if the smallest quantity is of the type $k_{T, i}$, the corresponding protojet is promoted to be a jet and removed from the list. Otherwise, if the smallest quantity is of the type $k_{T,(i, j)}$, the protojets are combined into a single protojet by summing up their four-vector components. The procedure is iterated over protojets until the list is empty. By construction, the algorithm is infrared safe and collinear safe to all orders in $\mathrm{pQCD}$. Since in this successive combination algorithm every particle is assigned to an unique jet, a prescription to solve situations of overlapping is not needed, allowing a well defined comparison to the theoretical predictions without introducing into the calculations the additional parameter $R_{\text {sep }}$. The final geometry of a jet defined by this kind of algorithms is likely to be more complex than from the cone algorithm, as illustrated figures 2.16 and 2.17. Note that, as figure 2.17 shows, the number of jets reconstructed and their variables depend on the jet algorithm applied.

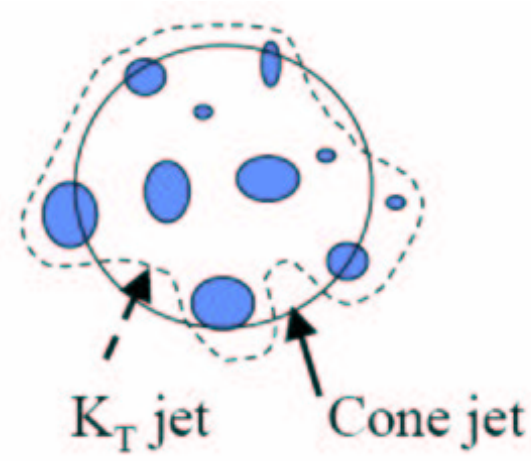

Figure 2.16: An illustration of the final jet geometry using the cone or the $k_{T}$ algorithm. 


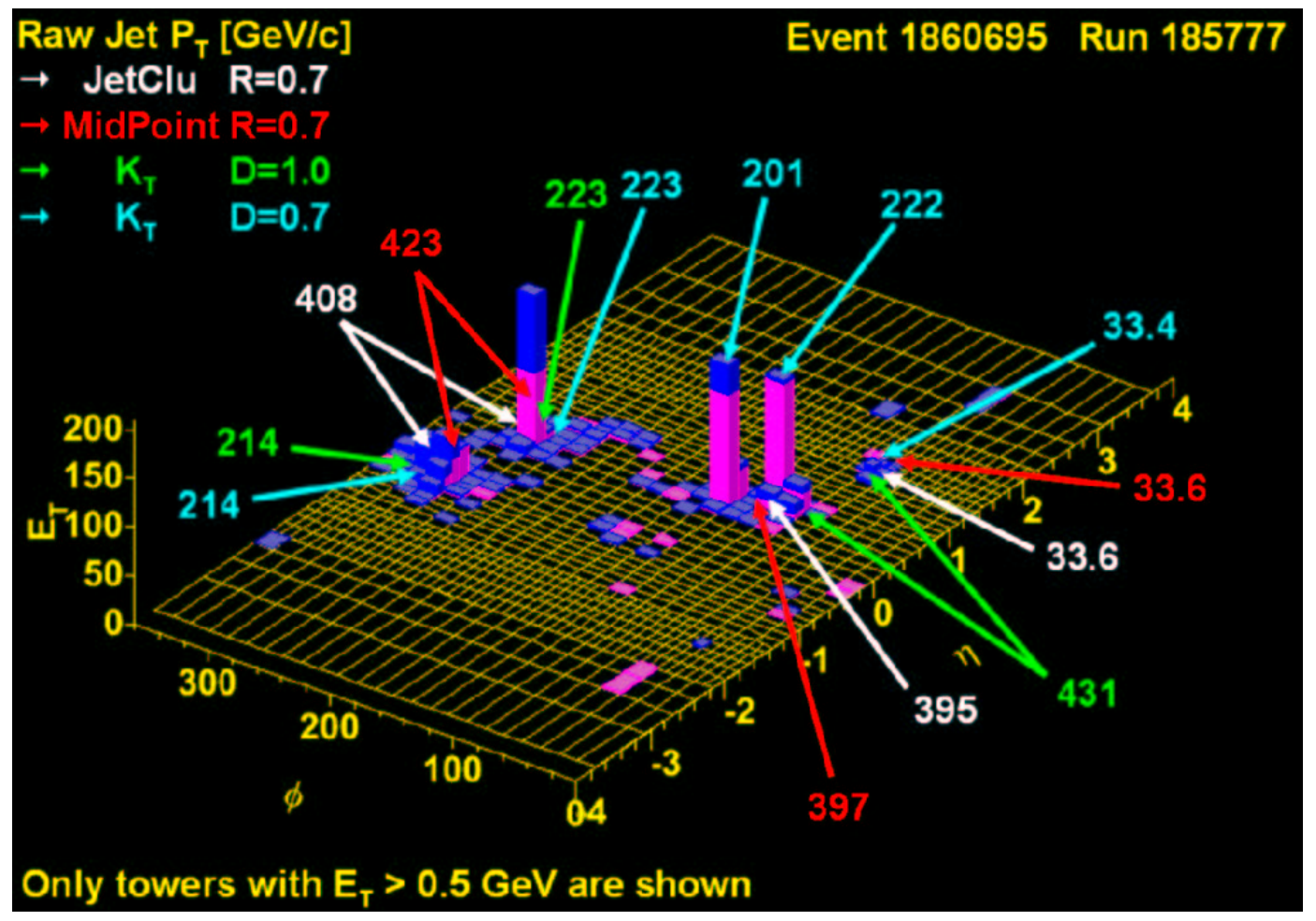

Figure 2.17: Lego plot of event 1860695 of run 185777. Towers with $\mathrm{E}_{\mathrm{T}}<0.5 \mathrm{GeV} / \mathrm{c}$ are not displayed. The number of jets and their energies, found using different algorithms, are reported.

\subsection{Jet Physics at Hadron Colliders}

The hadronic final states include contributions from the underlying event. These contributions consist of particles arising from beam-beam remnants, initial and final state radiation, and possible multiple parton interactions. The underlying event is in a non-perturbative QCD region. As in the description of the hadronization process, MC programs include models to simulate this soft component. These models are properly parametrized to describe experimental results. There is a special parametrization of the underlying events called PYTHIA-Tune A, where basically there is an increase of the initial-state radiation and the probability that the multiple parton interaction produces two gluons with colour connections to the nearest neighbours. The Tune A was a result of specific CDF Run I measurements [34]. Jet shape measurements are sensitive to the underlying modeling and a published CDF Run II results [35] (see appendix B) have shown that the jet shapes are better described by PYTHIA-Tune A. 
In hadron-hadron collisions at high instantaneous luminosity additional $p \bar{p}$ interactions per bunch crossing might be present, which produce soft contributions to the measured jet energies, that must be taken into account in the jet cross section measurements. 


\section{Chapter 3}

\section{CDF at Fermilab}

The data for the analysis described in this thesis was taken with the Collider Detector at Fermilab (CDF) located at the Fermi National Accelerator Laboratory. In the following sections, a brief introduction to the Tevatron Collider and a description of the CDF detector are given, with a particular attention to the detector components which are more relevant for the analysis.

\subsection{The Tevatron Collider}

The Tevatron Collider [36] located at the Fermi National Accelerator Laboratory (Fermilab) in Batavia (Illinois, USA) is a proton-antiproton $(p \bar{p})$ collider with a center-of-mass energy of $1.96 \mathrm{TeV}$. As shown in figure 3.1, this complex is constituted of five major accelerators and storage rings used in successive steps, as is explained in detail below, to produce, store and accelerate the particle up to $980 \mathrm{GeV}$.

The acceleration cycle starts with the production of protons from ionized hydrogen atoms $\mathrm{H}^{-}$, which are accelerated to $750 \mathrm{KeV}$ by a Cockroft-Walton electrostatic accelerator. Pre-accelerated hydrogen ions are then injected into the Linac where they are accelerated up to $400 \mathrm{MeV}$ by passing through a $150 \mathrm{~m}$ long chain of radio-frequency $(\mathrm{RF})$ accelerator cavities. To obtain protons, the $H^{-}$ions are passed through a carbon foil which strips their electrons off. Inside the Booster the protons are merged into bunches and accelerated up to an energy of $8 \mathrm{GeV}$ prior to entering the Main Injector. In the Main Injector, a synchrotron with a circumference of $3 \mathrm{~km}$, the proton 
bunches are accelerated further to an energy of $150 \mathrm{GeV}$ and coalesced ${ }^{1}$ together before injection into the Tevatron.

The production of the antiproton beam is significantly more complicated. The cycle starts with extracting a $120 \mathrm{GeV}$ proton beam from the Main Injector onto a stainless steel target. This process produces a variety of different particles, among which are antiprotons ${ }^{2}$. The particles come off the target at many different angles and they are focused into a beam line with a Lithium lens. In order to select only the antiprotons, the beam is sent through a pulsed magnet which acts as a charge-mass spectrometer. The produced antiprotons are then injected into the Debuncher, an $8 \mathrm{GeV}$ synchrotron, which reduces the spread in the energy distribution of the antiprotons. After that, the antiproton beam is directed into the Accumulator, a storage ring in the Antiproton Source, where the antiprotons are stored at an energy of $8 \mathrm{GeV}$ and stacked to $10^{12}$ particles per bunch. The antiproton bunches are then injected into the Main Injector and accelerated to $150 \mathrm{GeV}$.

\section{FERMILAB'S ACCELERATOR CHAIN}

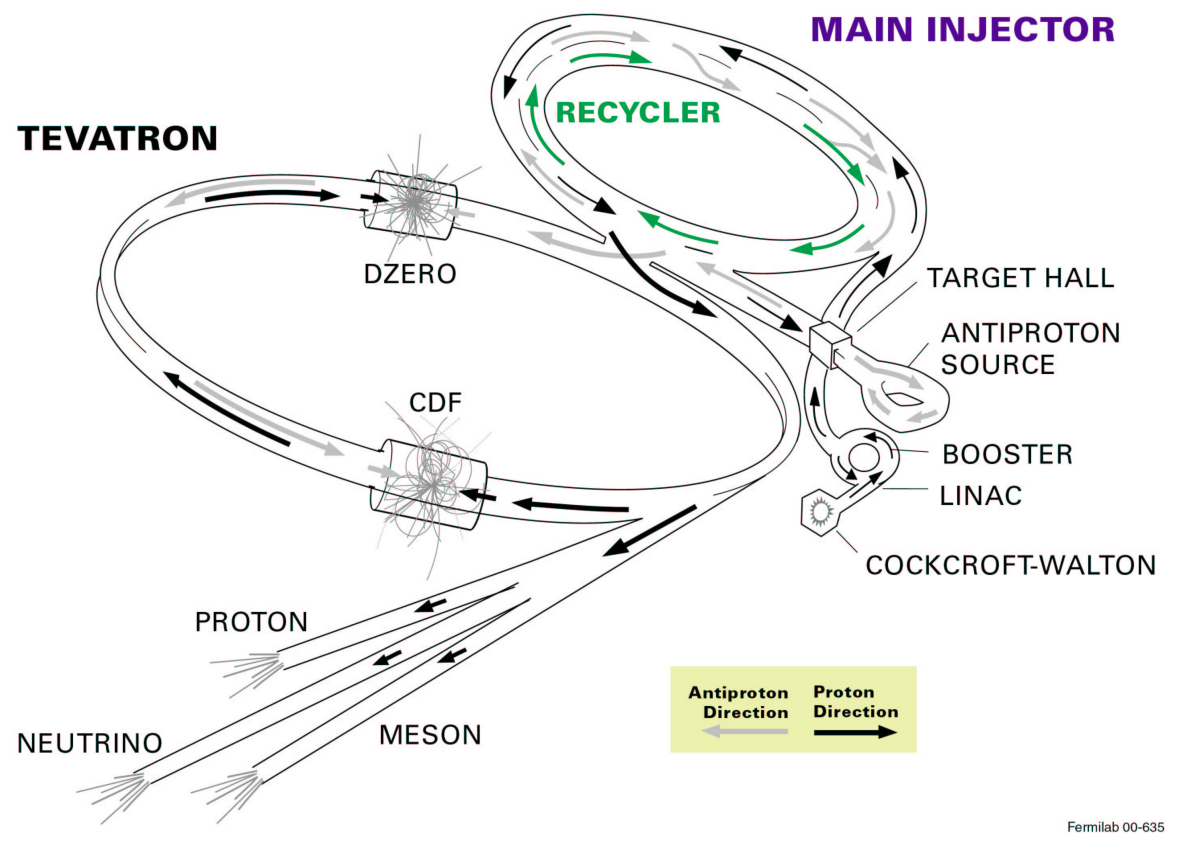

Figure 3.1: The Tevatron Collider Chain at Fermilab.

\footnotetext{
${ }^{1}$ coalescing is the process of merging proton bunches into one dense, high density, bunch

${ }^{2}$ The production rate, for $8 \mathrm{GeV}$ antiprotons, is about $18 \bar{p} / 10^{6} p$
} 
Finally, 36 proton and antiproton bunches are inserted into the Tevatron, a double acceleration ring of $1 \mathrm{~km}$ of radius, where their energy is increased up to $980 \mathrm{GeV}$. Proton and antiproton bunches circulate around the Tevatron in opposite directions bend by superconducting magnets and their orbits cross at the two collision points, B0 and D0, where interactions are observed by the CDF and D0 detectors, respectively.

In absence of a crossing angle or position offset, the luminosity in the Tevatron is given by the expression:

$$
L=\frac{f_{b c} N_{b} N_{p} N_{\bar{p}}}{2 \pi\left(\sigma_{p}^{2}+\sigma_{\bar{p}}^{2}\right)} F\left(\frac{\sigma_{l}}{\beta^{*}}\right)
$$

where $f_{b c}$ is the revolution frequency, $N_{b}$ is the number of bunches, $N_{p(\bar{p})}$ is the number of protons (antiprotons) per bunch, and $\sigma_{p(\bar{p})}$ is the transverse and longitudinal rms proton (antiproton) beam size at the interaction point. $F$ is a form factor with a complicated dependence on beta function, $\beta^{*}$, and the bunch length, $\sigma_{l}$. The beta function is a measure of the beam width, and it is proportional to the beam's $x$ and $y$ extent in phase space. Table 3.1 shows the design Run II accelerator parameters [37].

\begin{tabular}{|l|c|}
\hline Parameter & Run II \\
\hline number of bunches $\left(N_{b}\right)$ & 36 \\
revolution frequency $[\mathrm{MHz}]\left(f_{b c}\right)$ & 1.7 \\
bunch rms [m] $\sigma_{l}$ & 0.37 \\
bunch spacing [ns] & 396 \\
protons/bunch $\left(N_{p}\right)$ & $2.7 \times 10^{11}$ \\
antiprotons/bunch $\left(N_{\bar{p}}\right)$ & $3.0 \times 10^{10}$ \\
total antiprotons & $1.1 \times 10^{12}$ \\
$\beta^{*}[\mathrm{~cm}]$ & 35 \\
\hline
\end{tabular}

Table 3.1: Accelerator parameters for Run II configuration.

Figure 3.2 and 3.3 show, respectively, the evolution in the integrated luminosity, defined as $\mathcal{L}=\int L d t$, and the instantaneous luminosity delivered by Tevatron since the machine was turned on up to February 2006. The progressive increase in the integrated luminosity and the continuous records in the instantaneous luminosity ${ }^{3}$ prove the good performance of the accelerator.

\footnotetext{
${ }^{3}$ At February 2006, the record in the instantaneous luminosity was close to $1.8 \times 10^{32} \mathrm{~cm}^{-2} \mathrm{~s}^{-1}$.
} 


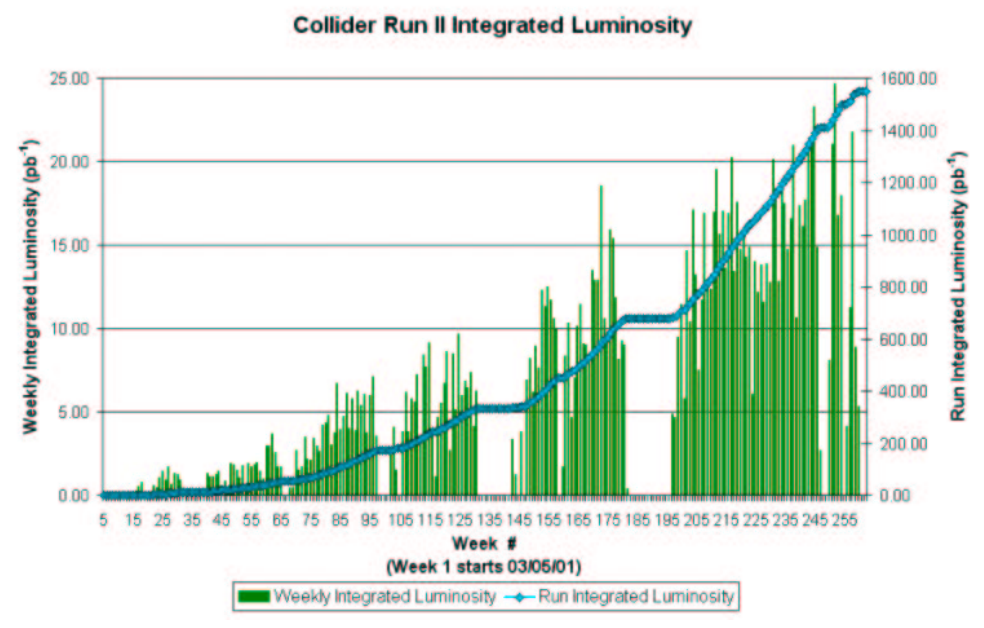

Figure 3.2: Tevatron Collider Run II Integrated Luminosity. The vertical blue bar shows each week's total luminosity as measured in $\mathrm{pb}^{-1}$. The diamond connected line displays the integrated luminosity.

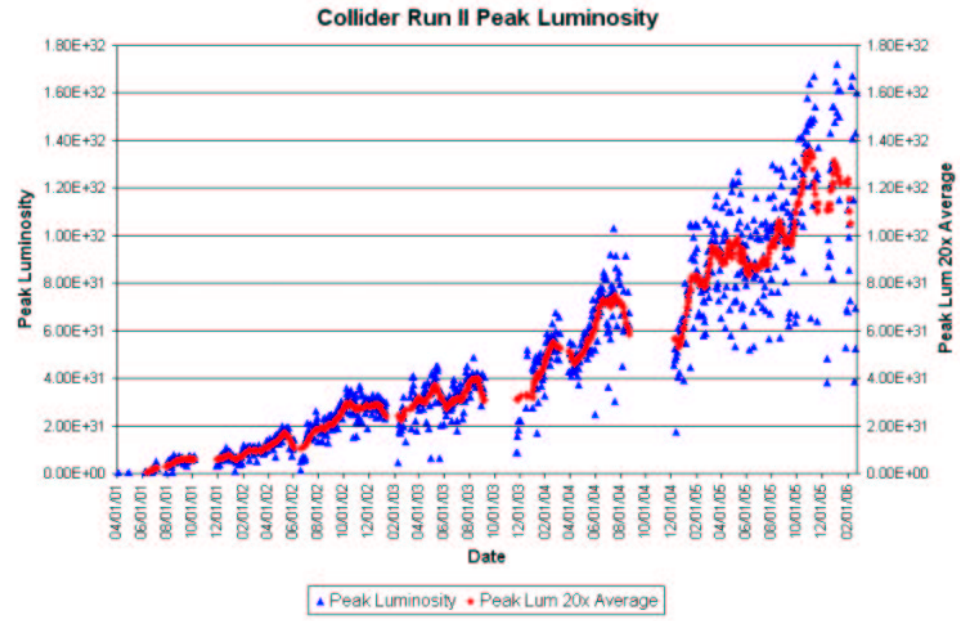

Figure 3.3: Tevatron Collider Run II Peak Luminosity. The blue squares show the peak luminosity at the beginning of each store and the red triangle displays a point representing the last 20 peak values averaged together. 


\subsection{CDF Run II detector}

The CDF Run II detector [38], in operation since 2001, is an azimuthally and forward-backward symmetric apparatus designed to study $p \bar{p}$ collisions at the Tevatron. It is a general purpose, cylindrical-shaped detector which combines:

- A tracking system, that provides a measurement of the charged particle momenta, event $\mathrm{z}$ vertex position and detects secondary vertices.

- A Time-of-Flight system, to identify charged particles.

- A non-compensated calorimeter system, with the purpose of measuring the energy of charged and neutral particles produced in the interaction.

- Drift chambers and scintillators to muon detection.

The detector is shown in figures 3.4 and 3.5. CDF uses a coordinate system with the positive $z$-axis lies along the direction of the incident proton beam, $\phi$ is the azimuthal angle, $\theta$ is the polar angle (measured from the detector center), and $p_{T}$ is the component of momentum in the transverse plane. A description of all the systems starting from the devices closest to the beam and moving outward is presented in the next sections, where the detectors most relevant in the analysis are explained in more detail.

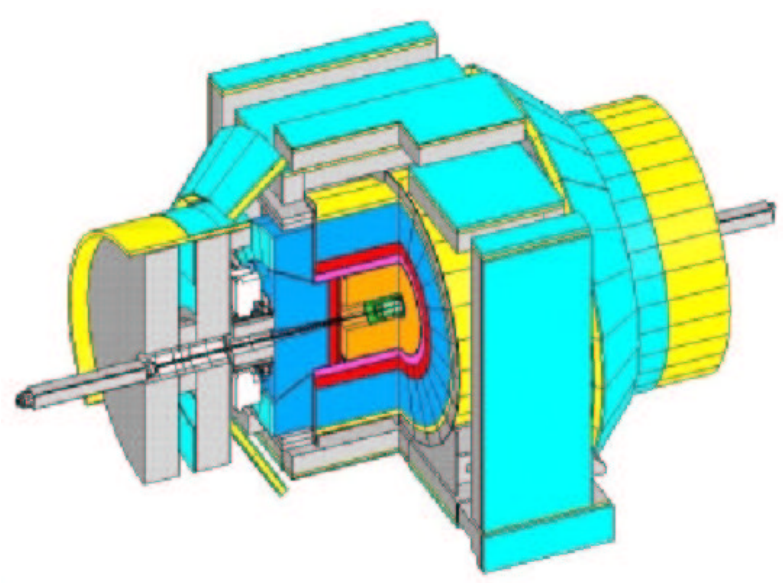

Figure 3.4: Isometric view of the CDF Run II detector. 


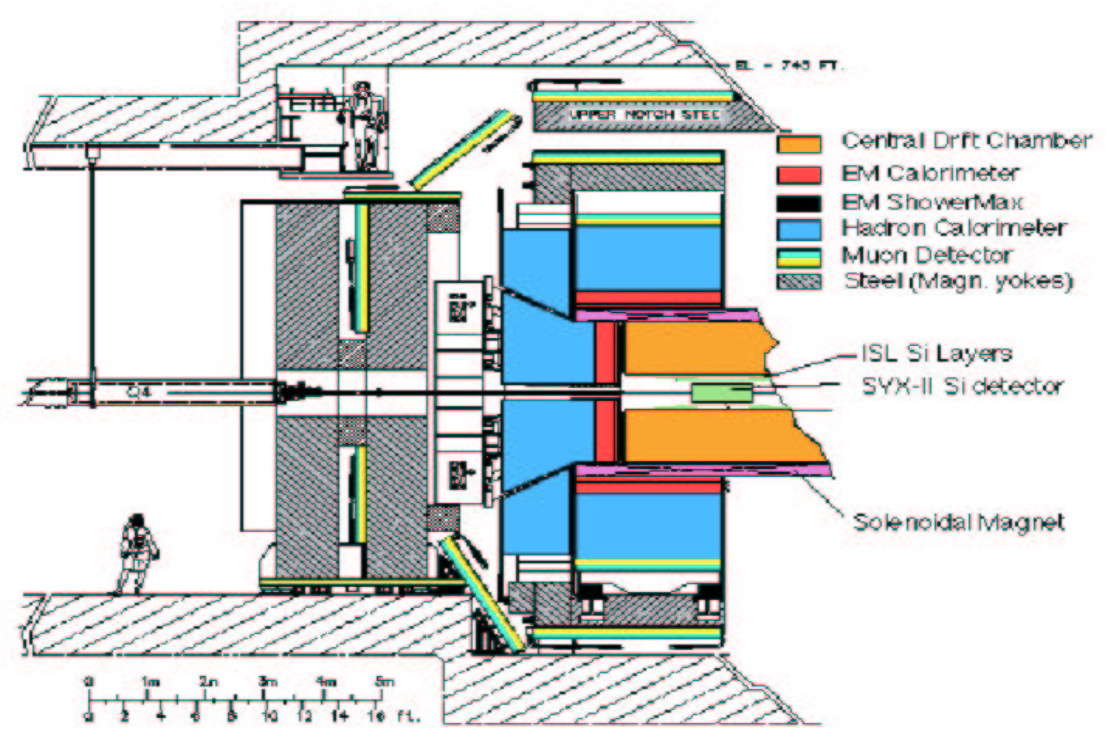

Figure 3.5: $r \times \eta$ side view of the CDF Run II detector.

\subsubsection{Tracking and Time of Flight systems}

The tracking and time of flight systems are contained in a superconducting solenoid, $1.5 \mathrm{~m}$ in radius and $4.8 \mathrm{~m}$ in length, which generates a $1.4 \mathrm{~T}$ magnetic field parallel to the beam axis.

The part of the tracking system closest to the beam pipe is a silicon microstrip detector [39], which is radiation-hard due its proximity to the beam. It extends from a radius of $r=1.5 \mathrm{~cm}$ from the beam line to $r=28 \mathrm{~cm}$, covering $|\eta|<2$ and it is constituted by eight layers in a barrel geometry. The innermost layer is a single-sided silicon microstrip detector called Layer 00 which provides a $r \times \phi$ position measurement. The first five layers after the Layer 00 consist in the Silicon Vertex Detector (SVXII) and the two outer layers comprise the Intermediate Silicon Layers system (ISL). These seven layers are made of double-sided silicon sensors, giving $r \times \phi$ and $z$ position information. The best position resolution achieved is $9 \mu \mathrm{m}$ in SVXII and the impact parameter resolution, including Layer 00 , arrives to $40 \mu \mathrm{m}$.

Surrounding the silicon detector is the Central Outer Tracker (COT) [40], the anchor of the CDF Run II tracking system. It is a $3.1 \mathrm{~m}$ long cylindrical drift chamber that covers the radial range from 40 to $137 \mathrm{~cm}(|\eta|<1)$. The COT contains 96 sense wire layers, which are radially 
grouped into eight "superlayers", as inferred from the end plate section shown in figure 3.6.

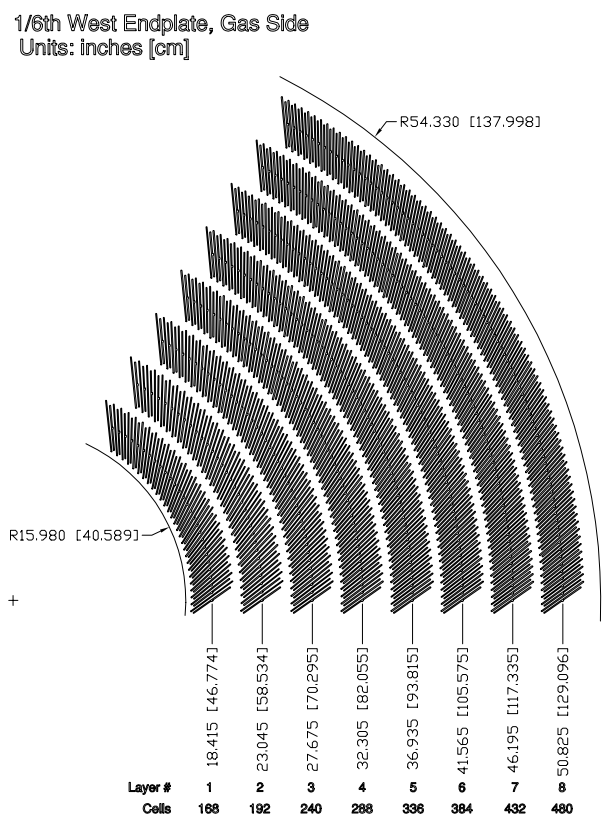

Figure 3.6: Layout of wire planes on a COT endplate.

Each superlayer is divided in $\phi$ into "supercells", and each supercell has 12 sense wires and a maximum drift distance that is approximately the same for all superlayers. Therefore, the number of supercells in a given superlayer scales approximately with the radius of the superlayer. The entire COT contains 30,240 sense wires. Approximately half the wires run along the $z$ direction ("axial"). The other half are strung at a small angle $\left(2^{\circ}\right)$ with respect to the $z$ direction ("stereo"). The combination of the axial and stereo information allows to measure the $z$ positions. Particles originated from the interaction point, which have $|\eta|<1$, pass through all 8 superlayers of the COT.

The supercell layout, shown in figure 3.7 for superlayer 2, consists of a wire plane containing sense and potential wires, for field shaping and a field (or cathode) sheet on either side. Both the sense and potential wires are $40 \mu \mathrm{m}$ diameter gold plated Tungsten. The field sheet is $6.35 \mu \mathrm{m}$ thick Mylar with vapor-deposited gold on both sides. Each field sheet is shared with the neighboring supercell. 


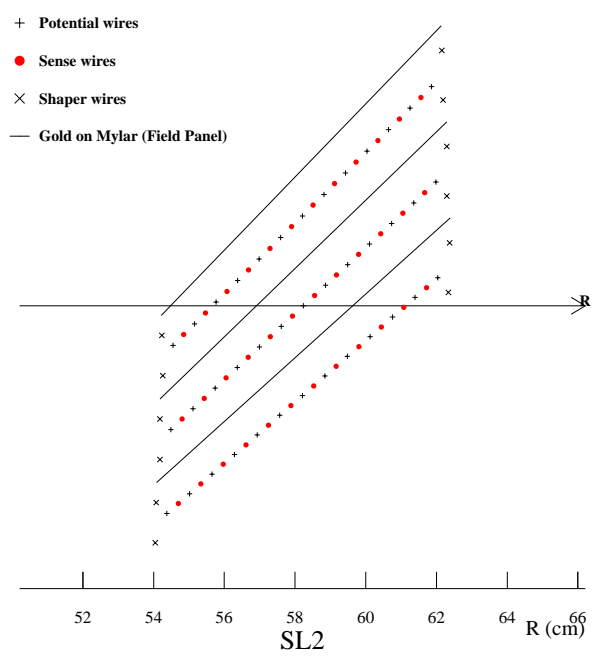

Figure 3.7: Layout of wires in a COT supercell.

The COT is filled with an Argon-Ethane gas mixture and Isopropyl alcohol (49.5:49.5:1). The mixture is chosen to have a constant drift velocity, approximately $50 \mu \mathrm{m} / \mathrm{ns}$ across the cell width and the small content of isopropyl alcohol is intended to reduce the aging and build up on the wires. When a charged particle passes through, the gas is ionized. Electrons drift toward the sense wires. The maximum electron drift time is approximately $100 \mathrm{~ns}$. Due to the magnetic field that the COT is immersed in, electrons drift at a Lorentz angle of $35^{\circ}$. The supercell is tilted by $35^{\circ}$ with respect to the radial direction to compensate for this effect. The momentum resolution of the tracks in the COT chamber depends on the $p_{T}$ and is measured to be approximately $0.15 \%$ $\mathrm{GeV} / \mathrm{c}^{-1}$, with corresponding hit resolution of about $140 \mu \mathrm{m}$ [41]. In addition to the measurement of the charged particle momenta, the COT is used to identify particles, with $p_{T}>2 \mathrm{GeV}$, based on $\mathrm{dE} / \mathrm{dx}$ measurements.

Just outside the tracking system, CDF II has a Time of Flight (TOF) detector [42]. It is a barrel of scintillator almost $3 \mathrm{~m}$ long located at $140 \mathrm{~cm}$ from the beam line with a total of 216 bars, each covering $1.7^{\circ}$ in $\phi$ and pseudorapidity range $|\eta|<1$. Particle identification is achieved by measuring the time of arrival of a particle at the scintillators with respect to the collision time. Thus, combining the measured time-of-flight and the momentum and path length, measured by the tracking system, the mass of the particle can then determined. The resolution in the time-offlight measurement is $\approx 100 \mathrm{ps}$ and it provides at least two standard deviation separation between $K^{ \pm}$and $\pi^{ \pm}$for momenta $\mathrm{p}<1.6 \mathrm{GeV} / \mathrm{c}$. 
As a summary, figure 3.8 illustrates the Tracking and Time of Flight systems.

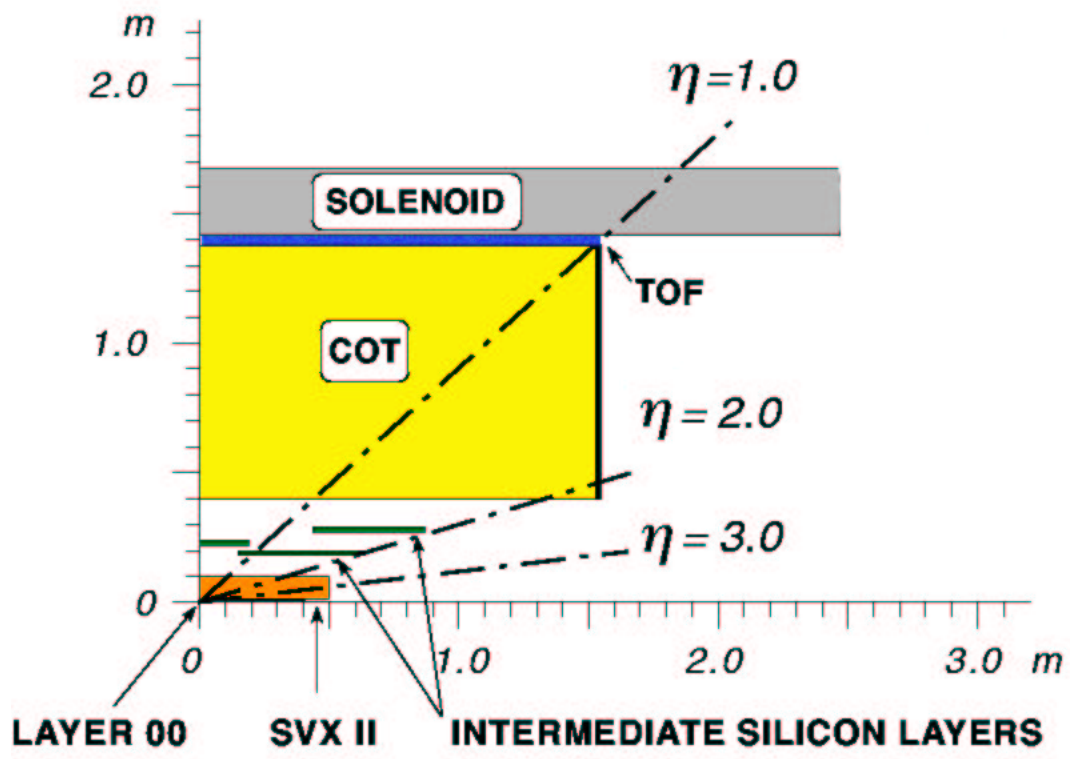

Figure 3.8: The CDF II tracker layout showing the different subdetector systems.

\subsubsection{Calorimeter system}

Surrounding the CDF tracking volume, outside of the solenoid coil, there is the calorimeter system. The different calorimeters that compose the system are scintillator-based detectors and segmented in projective towers (or wedges), in $\eta \times \phi$ space, that point to the interaction region. The total coverage of the system is $2 \pi$ in $\phi$ and about $|\eta|<3.64$ units in pseudorapidity.

The calorimeter system is divided in two regions: central and plug. The central calorimeter covers the region $|\eta|<1.1$ and is splitted in two halves at $|\eta|=0$. The forward plug calorimeters cover the angular range corresponding to $1.1<|\eta|<3.64$, as it is shown in figure 3.9. Due to this structure two "gaps" regions are found at $|\eta|=0$ and $|\eta| \sim 1.1$. 


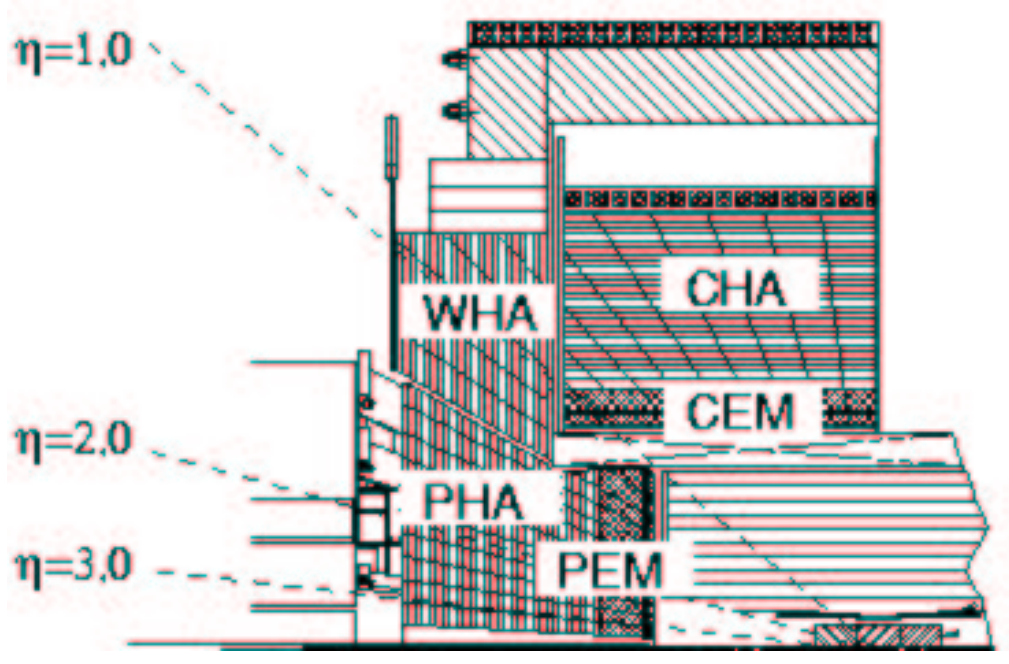

Figure 3.9: Elevation view of 1/4 of the CDF detector showering the components of the CDF calorimeter: CEM, CHA, WHA, PEM and PHA.

\section{Central Calorimeters}

The central calorimeters are constituted by 478 towers, each one is $15^{\circ}$ in azimuth by about 0.11 in pseudorapidity. Each wedge consists of an electromagnetic component backed by a hadronic section. In the central electromagnetic calorimeter (CEM) [43], the scintillators are interleaved with lead layers. The total material has a depth of 18 radiation lengths $\left(X_{0}\right)^{4}$. The central hadronic section (CHA) [44] has alternative layers of steel and scintillator and is 4.7 interaction lengths deep $\left(\lambda_{0}\right)^{5}$. The endwall hadron calorimeter (WHA), with similar construction than CHA, is located half of the detector behind the CEM/CHA and the other half behind the plug calorimeter. The function of the WHA detector is to provide a hadronic coverage in the region $0.9<|\eta|<$ 1.3. In the central calorimeter the light from the scintillator is redirected by two wavelength shifting (WLS) fibers, which are located on the $\phi$ surface between wedges covering the same pseudorapidity region, up through the lightguides into two phototubes (PMTs) per tower.

\footnotetext{
${ }^{4}$ The radiation length $X_{0}$ describes the characteristic amount of matter transversed, for high-energy electrons to lose all but $1 / e$ of its energy by bremsstrahlung, which is equivalent to $\frac{7}{9}$ of the length of the mean free path for pair $e^{+} e^{-}$production of high-energy photons. The average energy loss due to bremsstrahlung for an electron of energy $\mathrm{E}$ is related to the radiation length by $\left(\frac{d E}{d x}\right)_{\text {brems }}=-\frac{E}{X_{0}}$ and the probability for an electron pair to be created by a high-energy photon is $\frac{7}{9} X_{0}$.

${ }^{5} \mathrm{An}$ interaction length is the average distance a particle will travel before interacting with a nucleus.
} 
The energy resolution for each section was measured in the testbeam and, for a perpendicular beam incident, can be parameterized as:

$$
(\sigma / E)^{2}=\left(\sigma_{1} / \sqrt{E}\right)^{2}+\left(\sigma_{2}\right)^{2},
$$

where the first term comes from sampling fluctuations and the photostatistics of PMTs, and the second term comes from the non-uniform response of the calorimeter. In the CEM, the energy resolution for high energy electrons and photons is $\frac{\sigma\left(E_{T}\right)}{E_{T}}=\frac{13.5 \%}{\sqrt{E_{T}}} \oplus 1.5 \%$, where $E_{T}=E \sin \theta$ expressed in $\mathrm{GeV}$ being $\theta$ the beam incident angle. Charge pions were used to obtain the energy resolution in the CHA and WHA detectors that are $\frac{\sigma\left(E_{T}\right)}{E_{T}}=\frac{50 \%}{\sqrt{E_{T}}} \oplus 3 \%$ and $\frac{\sigma\left(E_{T}\right)}{E_{T}}=\frac{75 \%}{\sqrt{E_{T}}} \oplus 4 \%$, respectively.

\section{Plug Calorimeters}

One of the major components upgraded for the Run II was the plug calorimeter [45]. The new plug calorimeters are built with the same technology that the central components and replace the Run I gas calorimeters in the forward region. The $\eta \times \phi$ segmentation depends on the tower pseudorapidity coverage. For towers in the region $|\eta|<2.1$, the segmentation is $7.5^{\circ}$ in $\phi$ and from 0.1 to 0.16 in the pseudorapidity direction. For more forward wedges, the segmentation change to $15^{\circ}$ in $\phi$ and about 0.2 to 0.6 in $\eta$.

As in the central calorimeters, each wedge consists of an electromagnetic (PEM) and a hadronic section (PHA). The PEM, with 23 layers composed for lead and scintillator, has a total thickness of about $21 X_{0}$. The PHA is a steel/scintillator device with a depth of about $7 \lambda_{0}$. In both sections the scintillator tiles are read out by WLS fibers embedded in the scintillator. The WLS fibers carry the light out to PMTs tubes located on the back plane of each endplug. Unlike to the central calorimeters, each tower is only read out by one PMT.

Testbeam measurements determined that the energy resolution of the PEM for electrons and photons is $\frac{\sigma}{E}=\frac{16 \%}{\sqrt{E}} \oplus 1 \%$. The PHA energy resolution is $\frac{\sigma}{E}=\frac{80 \%}{\sqrt{E}} \oplus 5 \%$ for charged pions that do not interact in the electromagnetic component. Table 3.2 summarizes the calorimeter subsystems and their characteristics.

The central and forward parts of the calorimeter have their own shower profile detectors: shower maximum and preshower detectors. The Central Shower Maximum (CES) and the Plug Shower Maximum (PES) are positioned at about $6 X_{0}$, while the Central Preradiator (CPR) and the Plug Preradiator (PPR) are located at the inner face of the calorimeters. These detectors help on particle identification, separating $e^{ \pm}, \gamma s$ and $\pi^{0} s$. 


\begin{tabular}{|l|c|c|c|}
\hline Calorimeter & Coverage & Thickness & Energy resolution $(\mathrm{E}$ expressed in GeV) \\
\hline CEM & $|\eta|<1.1$ & $18 X_{0}$ & $\frac{13.5 \%}{\sqrt{E_{T}}} \oplus 2 \%$ \\
CHA & $|\eta|<0.9$ & $4.7 \lambda_{0}$ & $\frac{50 \%}{\sqrt{E_{T}}} \oplus 3 \%$ \\
WHA & $0.9<|\eta|<1.3$ & $4.7 \lambda_{0}$ & $\frac{75 \%}{\sqrt{E_{T}}} \oplus 4 \%$ \\
\hline PEM & $1.1<|\eta|<3.6$ & $21 X_{0}, 1 \lambda_{0}$ & $\frac{16 \%}{\sqrt{E}} \oplus 1 \%$ \\
PHA & $1.2<|\eta|<3.6$ & $7 \lambda_{0}$ & $\frac{80 \%}{\sqrt{E}} \oplus 5 \%$ \\
\hline
\end{tabular}

Table 3.2: CDF II Calorimeter subsystems and characteristics. The energy resolution for the EM calorimeter is given for a single incident electron and that for the hadronic calorimeter for a single incident pion.

\subsubsection{Muons system}

The muon system, which consists of sets of drift chambers and scintillators, is installed beyond the calorimetry system as the radially outermost component of CDF Run II detector ( $r \sim 3.5 \mathrm{~m})$. The muon system $[46,47]$ is divided into different subsystems, that combined coverage the pseudorapidity range $|\eta|<2.0$ : the Central Muon Detector (CMU), the Central Muon Upgrade Detector (CMP/CSP), the Central Muon Extension Detector (CMX/CSX) and the Intermediate Muon Detector (IMU). The $z$ and $\phi$ coordinates muon candidate are often provided by the chambers while the scintillator detectors are used for triggering and spurious signal rejection.

\subsection{Luminosity Measurement}

\subsubsection{CLC detector}

In CDF, the beam luminosity is determined using gas Cherenkov counters (CLC) [48] located in the pseudorapidity region $3.7<|\eta|<4.7$, which measure the average number of inelastic interaction per bunch crossing. Each module consists of 48 thin, gas-filled, Cherenkov counters. The counters are arranged around the beam pipe in three concentric layers, with 16 counters each, and pointing to the center of the interaction region. The cones in the two outer layers are about $180 \mathrm{~cm}$ long and the inner layer counters, closer to the beam pipe, have a length of $110 \mathrm{~cm}$. The Cherenkov light is detected with fast photomultiplier tubes. 


\subsubsection{Measurement of the luminosity}

The average number of primary interactions, $\mu$, is related to the instantaneous luminosity, $\mathcal{L}$, by the expression:

$$
\mu \cdot f_{b c}=\sigma_{t o t} \cdot \mathcal{L}
$$

where $f_{b c}$ is the bunch crossings frequency at Tevatron, on average $1.7 \mathrm{MHz}$ for $36 \times 36$ bunch operations, and $\sigma_{t o t}$ is the total $p \bar{p}$ cross section.

Since the CLC is not sensitive at all to the elastic component of the $p \bar{p}$ scattering, the equation 3.3 can be rewritten using the inelastic cross section, $\sigma_{i n}$, as:

$$
\mathcal{L}=\frac{\mu \cdot f_{b c}}{\sigma_{\text {in }}},
$$

where now $\mu$ is the average number of inelastic $p \bar{p}$ interactions. The method used in CDF for the luminosity measurement is based on the counting of empty crossings [49]. This method determines $\mu$ by measuring the first bin of the distribution which corresponds to the probability of having zero inelastic interactions, $P_{0}$, through the relation:

$$
P_{0}(\mu)=e^{-\mu}
$$

which is correct if the acceptance of the detector and its efficiency were $100 \%$. In practice, there are some selection criteria, $\alpha$, to define an "interaction". An "interaction" is defined as a $p \bar{p}$ crossing with hits above a fixed threshold on both sides of the CLC detector. Therefore, an empty crossing is a $p \bar{p}$ crossing with no interactions. Given these selection criteria, the experimental quantity $P_{0}$, called $P_{0}^{\exp }\{\alpha\}$, is related to $\mu$ as:

$$
P_{0}^{e x p}\{\mu ; \alpha\}=\left(e^{\varepsilon_{\omega} \cdot \mu}+e^{-\varepsilon_{e} \cdot \mu}-1\right) \cdot e^{-\left(1-\varepsilon_{0}\right) \cdot \mu},
$$

where the acceptances $\varepsilon_{0}$ and $\varepsilon_{\omega / e}$ are, respectively, the probability to have no hits in the combined east and west CLC modules and the probability to have at least one hit exclusively in west/east CLC module. The evaluation of these parameters is based on Monte Carlo simulations, and typical values are $\varepsilon_{0}=0.07$ and $\varepsilon_{\omega / e}=0.12$. 
To obtain the luminosity measurement using the equation 3.4, the value of $\sigma_{\text {in }}$ is still needed. At the beginning of Run II, an extrapolation to $2 \mathrm{TeV}$ of the value measured at $\sqrt{s}=1.8 \mathrm{TeV}$ by CDF [50] was used. The cross section would be $\sigma_{i n}=60.4 \mathrm{mb}$. To facilitate the comparison of $\mathrm{CDF}$ and D0 cross section measurements in Run II, the collaborations agreed to use a common inelastic cross section [51], $\sigma_{i n}=59.3 \mathrm{mb}$ that is about $1.9 \%$ smaller than previous value. Since CDF never modified the value used online and offline, the CDF quoted luminosity is multiplied offline by a factor of 1.019 .

Different sources of uncertainties have been taken into account to evaluate the systematic uncertainties on the luminosity measurement [52]. The dominated contributions are related to the detector simulation and the event generator used, and have been evaluated to be about $3 \%$. The total systematic uncertainty in the CLC luminosity measurements is $5.8 \%$, which includes uncertainties on the measurement (4.2\%) and on the inelastic cross section value (4\%).

\subsection{Trigger and Data Acquisition}

The average interaction rate at the Tevatron is $1.7 \mathrm{MHz}$ for $36 \times 36$ bunches. In fact, the actual interaction rate is higher because the bunches circulate in three trains of 12 bunches in each group spaced $396 \mathrm{~ns}$ which leads to a crossing rate of $2.53 \mathrm{MHz}$. The interaction rate is orders of magnitude higher than the maximum rate that the data acquisition system can handle. Furthermore, the majority of collisions are not of interest. This leads to implementation of a trigger system that preselects events online and decides if the corresponding event information is written to tape or discarded.

The CDF trigger system consists of three trigger levels, see figures 3.10 and 3.11, where the first two levels are hardware based and the third one is a processor farm. The decisions taken by the system are based on increasingly more complex event information. The two harward levels are monitored and controlled by the Trigger Supervisor Interface (TSI), which distributes signals from the different sections of the trigger and DAQ system, a global clock and bunch crossing signal. 


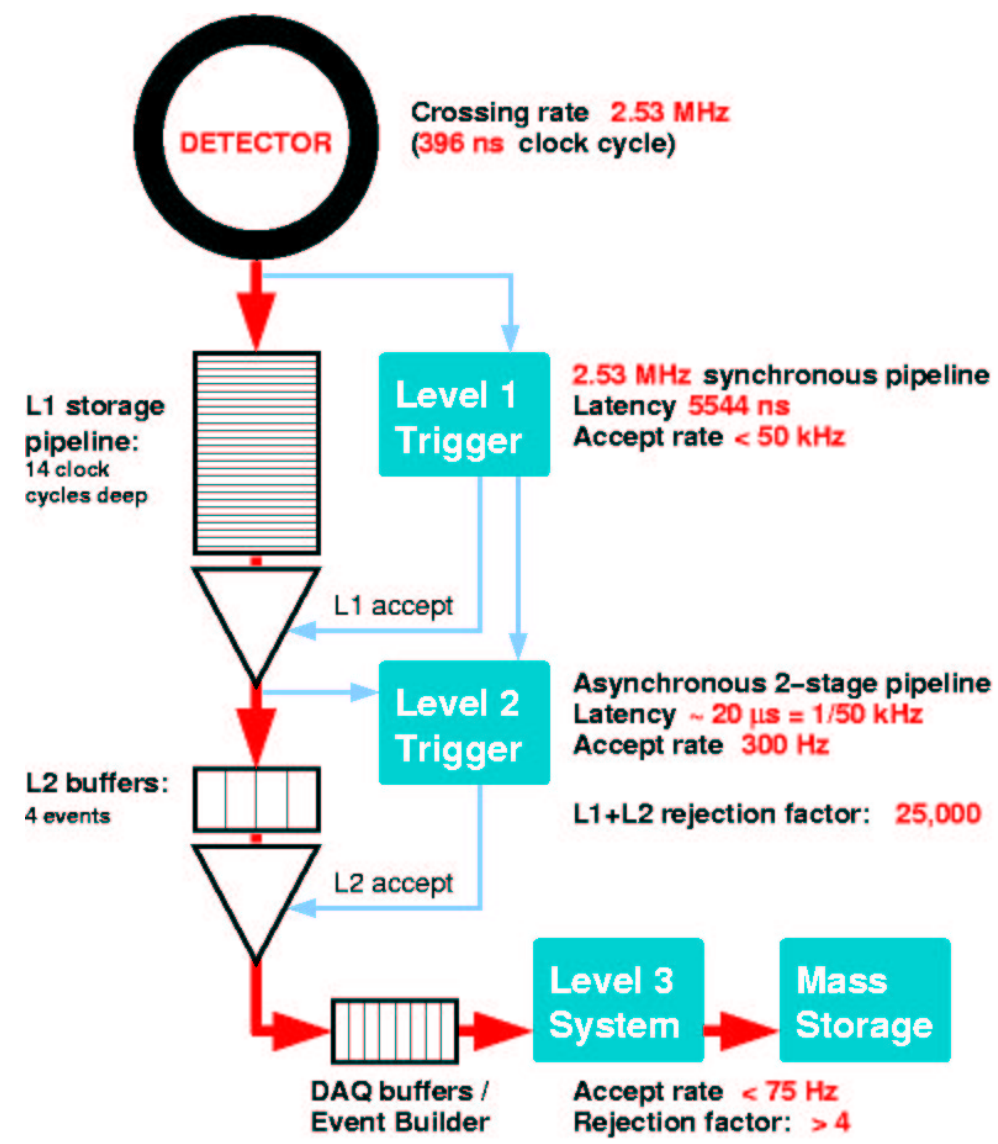

Figure 3.10: Block diagram showing the global trigger and DAQ systems at CDF II. 


\section{RUN II TRIGGER SYSTEM}

Detector Elements

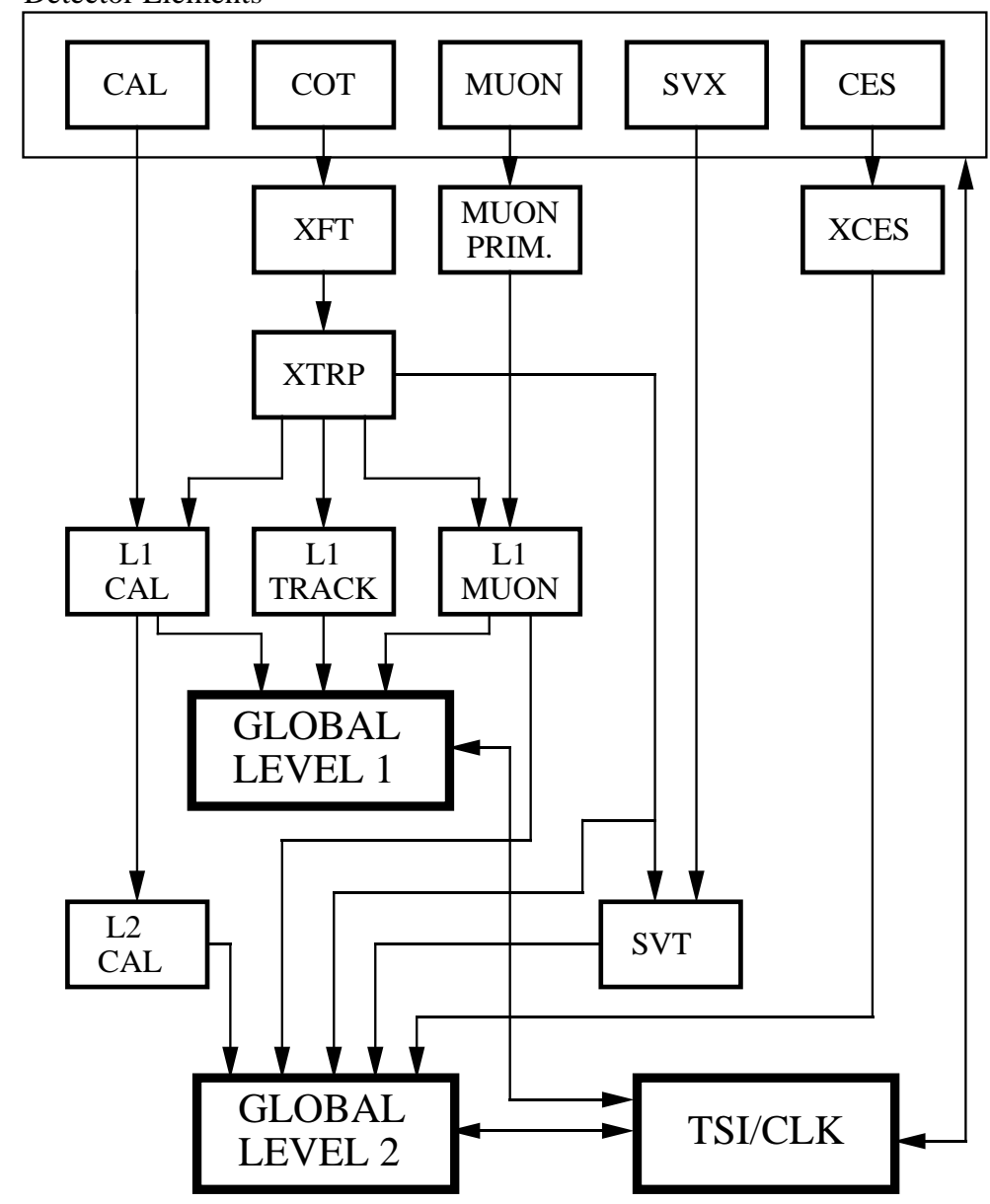

PJW 9/23/96

Figure 3.11: Block diagram showing the Level 1 and Level 2 trigger systems. 


\subsubsection{Level 1 trigger}

The Level 1 trigger is a synchronous system with an event read and a decision made every beam crossing. The depth of the $\mathrm{L} 1$ decision pipeline is expected to be approximately $4 \mu \mathrm{s}$ (L1 latency). The L1 buffer must be at least as deep as this processing pipeline or the data associated with a particular L1 decision would be lost before the decision is made. The L1 buffer is 14 crossing deep (5544 ns at $396 \mathrm{~ns}$ bunch spacing) to provide a margin for unanticipated increases in L1 latency. The Level 1 reduces the event rates from $2.53 \mathrm{MHz}$ to less than $50 \mathrm{kHz}$.

The Level 1 hardware consists of three parallel processing streams which feed inputs of the Global Level 1 decision unit. One stream finds calorimeter based objects (L1 CAL), another finds muons (L1 MUON), while the third one finds tracks in the COT (L1 TRACK). Since the muons and the calorimeter based objects require the presence of a track pointing at the corresponding outer detector element, the tracks must be sent to the calorimeter and muon streams as well as the track only stream.

- The L1 CAL calorimeter trigger is employed to detect electrons, photons, jets, total transverse energy and missing transverse energy, $E_{T}^{m i s s}$. The calorimeter triggers are divided into two types: object triggers (electron, photons and jets) and global triggers ( $\sum E_{T}$ and $E_{T}^{\text {miss }}$ ). The calorimeter towers are summed into trigger towers of $15^{\circ}$ in $\phi$ and by approximately 0.2 in $\eta$. Therefore, the calorimeter is divided in $24 \times 24$ towers in $\eta \times \phi$ space [53]. The object triggers are formed by applying thresholds to individual calorimeter trigger towers, while thresholds for the global triggers are applied after summing energies from all towers.

- The L1 TRACK trigger is designed to detect tracks on the COT. An eXtremely Fast Tracker (XFT) [54] uses hits from 4 axial layers of the COT to find tracks with a $p_{T}$ greater than some threshold $(\sim 2 \mathrm{GeV} / \mathrm{c})$. The resulting track list is sent to the extrapolation box (XTRP)[55] that distributes the tracks to the Level 1 and Level 2 trigger subsystems.

- L1 MUON system uses muon primitives, generated from various muon detector elements, and XFT tracks extrapolated to the muon chambers by the XTRP to form muon trigger objects. For the scintillators of the muon system, the primitives are derived from single hits or coincidences of hits. In the case of the wire chambers, the primitives are obtained from patterns of hits on projective wire with the requirement that the difference in the arrival times of signals be less than a present threshold. This maximum allowed time difference imposes a minimum $p_{T}$ requirement for hits from a single tracks. 
Finally, the Global Level 1 makes the L1 trigger decision based on the quantity of each trigger object passed to it.

\subsubsection{Level 2 trigger}

The Level 2 trigger is an asynchronous system which processes events that have received a L1 accept in FIFO (First In, First Out) manner. It is structured as a two stage pipeline with data buffering at the input of each stage. The first stage is based on dedicated hardware processor which assembles information from a particular section of the detector. The second stage consists of a programmable processors operating on lists of objects generated by the first stage. Each of the L2 stages is expected to take approximately $10 \mu$ s giving a latency of approximately $20 \mu \mathrm{s}$. The L2 buffers provide a storage of four events. After the Level 2, the event rate is reduced to about $300 \mathrm{~Hz}$.

In addition of the trigger primitives generated for L1, data for the L2 come from the shower maximum strip chambers in the central calorimeter and the $r \times \phi$ strips of the SVX II. There are three hardware systems generating primitives at Level 2: Level 2 cluster finder (L2CAL), shower maximum strip chambers in the central calorimeter (XCES) and the Silicon Vertex Tracker (SVT).

- The L2CAL hardware carries out the hardware cluster finder functions. It receives trigger tower energies from the L1 CAL and applies seed and "shoulder" thresholds for cluster finding. It is basically designed for jet triggers. More details about the cluster finder algorithm in section 4.2.1.

- The shower maximum detector provides a much better spacial resolution than a calorimeter wedge. The XCES boards perform sum of the energy on groups of four adjacent CES wires and compare them to a threshold (around $4 \mathrm{GeV}$ ). This information is matched to XFT tracks to generate a Level 2 trigger. This trigger hardware provides a significant reduction in combinatorial background for electrons and photons.

- Silicon Vertex Tracker [56] uses hits from the $r \times \phi$ strips of the SVX II and tracks from the XFT to find tracks in SVX II. SVT improves on the XFT resolution for $\phi$ and $p_{T}$ and adds a measurement of the track impact parameter $d_{0}$. Hereby the efficiency and resolution are comparable to those of the offline track reconstruction. The SVT enables triggering on displaced tracks, that have a large impact parameter $d_{0}$. 


\subsubsection{Level 3 trigger}

When an event is accepted by the Level 2 trigger, its data become available for readout distributed over a couple of hundred of VME Readout Buffers (VRBs). The event has to be assembled from pieces of data from the L2 system into complete events, this is the purpose of the Event Builder. It is divided into 16 sub-farms, each consisting of 12-16 processor nodes. Once the event is built, it is sent to one place in the Level 3 farm. The Level 3 trigger reconstructs the event following given algorithms. These algorithms take advantage of the full detector information and improved resolution not available to the lower trigger levels. This includes a full 3-dimensional track reconstruction and tight matching of tracks to calorimeter and muon-system information. Events that satisfy the Level 3 trigger requirements are then transfered onward to the Consumer Server/Data Logger (CSL) system for storage first on disk and later on tape. The average processing time per event in Level 3 is on the order of one second. The Level 3 leads to a further reduction in the output rate, a roughly $50 \mathrm{~Hz}$.

A set of requirements that an event has to fulfill at Level 1, Level 2 and Level 3 constitutes a trigger path. The CDF II trigger system implements about 150 trigger paths. An event will be accepted if it passes the requirements of any one of these paths and, depending of the trigger path, it will be storage in a trigger dataset. A complete description of the different datasets at CDF Run II can be found in [57].

In addition to impose the trigger requirements to select out interesting physics events, trigger can be prescaled in the different levels. To prescale means to accept only a predetermined fraction of events selected by a given trigger path.

\subsection{Data Quality Monitoring}

The CDF experiment has implemented a system to check the data quality of all subsystems in real-time as well as after data are fully processed. This Data Quality Monitoring marks the status of all the detector components for each run. For more information see appendix A. 


\section{Chapter 4}

\section{Inclusive Jet Cross Section Measurement}

In this chapter, the complete analysis of the inclusive jet cross section measurement using the longitudinally invariant $k_{T}$ algorithm is described. The measurement is performed for jets with $p_{T}^{\text {jet }} \geq 54 \mathrm{GeV} / \mathrm{c}$ in five different jet rapidity regions up to $\left|y^{j e t}\right|=2.1$. The results are based on $1 \mathrm{fb}^{-1}$ of CDF Run II data. The chapter starts explaining the event selection criteria, describes the corrections applied to jet measurements, and finishes with a detailed discussion on the systematic uncertainties.

\subsection{Event selection}

The event selection is done over data samples that include events taken with the CDF Run II detector from the 2001 Summer up to November 2005. This corresponds to a luminosity of approximately $1.2 \mathrm{fb}^{-1}$.

\subsubsection{Run selection}

The event selection starts considering only runs included in the QCD good run list (see appendix A), where it is required that the calorimeter system and COT are working properly during the run. In addition, some runs with undetermined trigger prescale factors have been excluded: 
- runs $192384,192386,195452$ and 206951 for which the prescale was changed during the runs.

- runs $<147870$ only for ST5 dataset for which non-constant L2 prescales were used during data taking.

The remaining integrated luminosity is $0.99 \mathrm{fb}^{-1}$ for JET 20 to JET 100 datasets and $0.950 \mathrm{fb}^{-1}$ for ST5 sample. It should be indicated that the ST5 sample is only used for trigger efficiency studies (see section 4.2 for datasets description).

\subsubsection{Jet selection criteria}

All the events with at least one jet in the rapidity region $\left|y^{j e t}\right|<2.1$ and with an uncorrected transverse momentum, $p_{T, C A L}^{\text {jet }}$, about $10 \mathrm{GeV} / \mathrm{c}$ are selected. In this analysis jets are defined with the longitudinally invariant $k_{T}$ algorithm (see section 2.8.3). A D parameter of 0.7 is used. For jets with $0.1<\left|y^{\text {jet }}\right|<0.7$, measurements with $\mathrm{D}$ set to 0.5 and 1.0 are also performed.

In order to remove beam related background and cosmic rays, the events are required to have at least one primary vertex with $\left|V_{Z}\right| \leq 60 \mathrm{~cm}$ and a missing $E_{T}$ significance value below a threshold function, $T\left[p_{T, C A L}^{j e t}(\right.$ Leading jet $\left.)\right]$. The missing $E_{T}$ significance is defined as the ratio $E_{T}^{\text {miss }} / \sqrt{\Sigma E_{T}}$, where $E_{T}^{\text {miss }}$ denotes the missing transverse energy and $\Sigma E_{T}$ the total transverse energy. The threshold function is obtained from MC studies to maximize the efficiency and is defined as:

$$
T\left[p_{T, C A L}^{\text {jet }}(\text { Leading jet })\right]=\operatorname{minimum}\left(2+5 / 400 \cdot p_{T, C A L}^{\text {jet }}(\text { Leading Jet }), 7\right),
$$

with $T$ in $\mathrm{GeV}^{1 / 2}$ and $p_{T, C A L}^{\text {jet }}$ in $\mathrm{GeV} / \mathrm{c}$. As figure 4.1 illustrates, the transition to a constant cut of $7 \mathrm{GeV}^{1 / 2}$ occurs for $p_{T, C A L}^{\text {jet }}=400 \mathrm{GeV} / \mathrm{c}$. The efficiency is kept above $95 \%$, see figure 4.2 .

It should be mentioned that the $p_{T}$ binning has been selected according to the jet energy resolution and increases as $p_{T}^{\text {jet }}$ increases to accommodate the limitation in statistics at very high $p_{T}^{\text {jet }}$. 


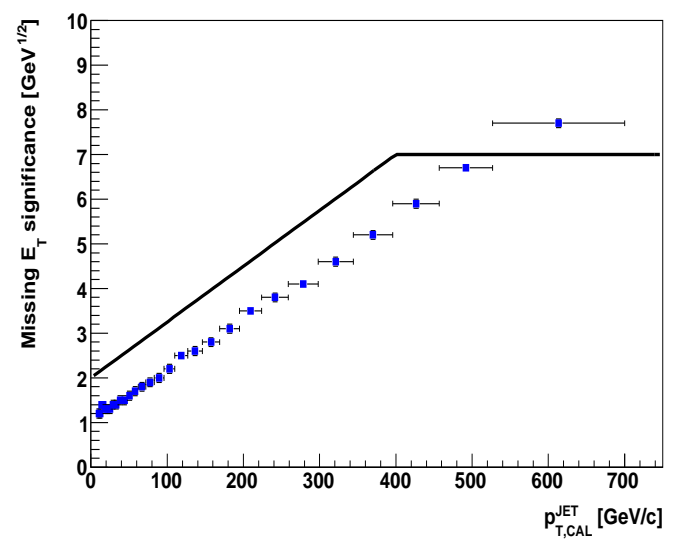

Figure 4.1: Missing $E_{T}$ significance cut study using MC. The points represent the value of Missing $E_{T}$ significance if a cut, that keep a 95\% efficiency, is applied. The solid line shows the threshold function used in the analysis.
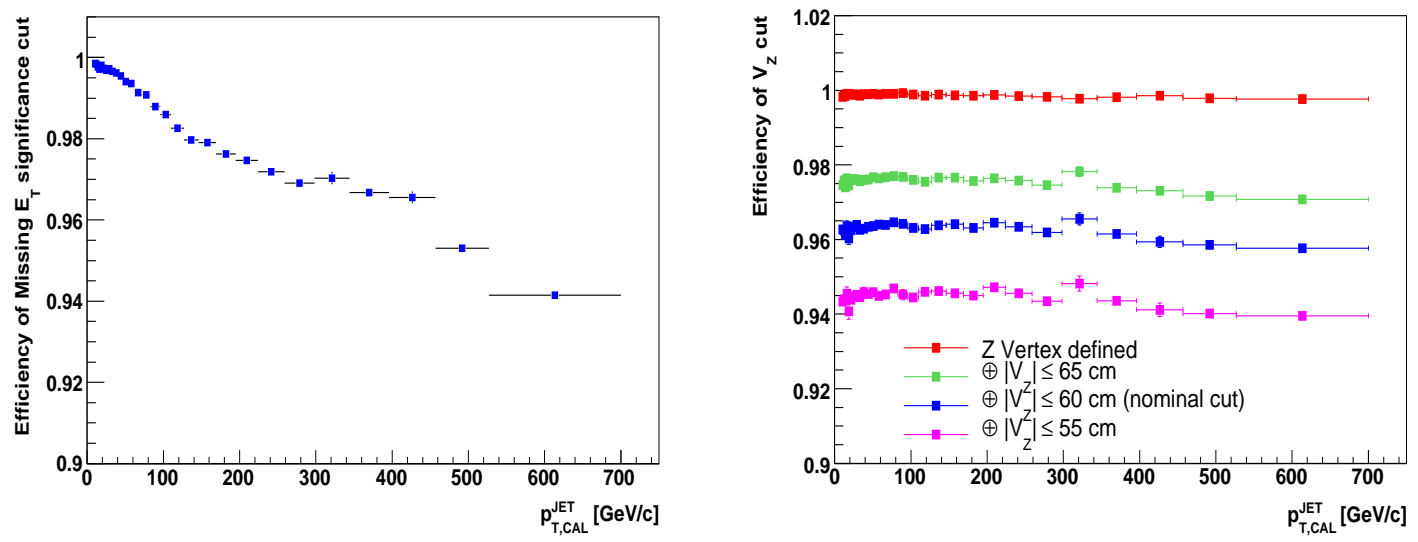

Figure 4.2: Efficiency of the missing $E_{T}$ significance cut (right) and the primary vertex cut (left) vs the $p_{T}^{j e t}$. The efficiency of the vertex cut plot shows how the efficiency changes if different cuts are applied. 
The different rapidity regions have been chosen taking into account the layout of the CDF calorimeters according to dijet balance studies, see figure 4.3. The five regions are:

- $0<\left|y^{j e t}\right|<0.1: 90^{\circ}$ gap.

- $0.1<\left|y^{j e t}\right|<0.7$ : central calorimeter.

- $0.7<\left|y^{\text {jet }}\right|<1.1$ : central calorimeter and $30^{\circ}$ gap.

- $1.1<\left|y^{j e t}\right|<1.6: 30^{\circ}$ gap and plug calorimeter.

- $1.6<\left|y^{\text {jet }}\right|<2.1$ : plug calorimeter.

In the whole rapidity range the jets are well contained inside the calorimeter and contributions from proton and antiproton remnants inside the jet are not expected. In addition, the comparison between data and MC in the dijet balance plot shows that they agree for $\left|\eta^{j e t}\right|<2$.1. At higher $\eta$ a disagreement bigger than $2 \%$ is observed. This could be partially attributed to defects on the simulation of the energy losses across the calorimeter edges, and could also reflect the presence of beam-halo related contributions that affect the most-forward calorimeter towers.

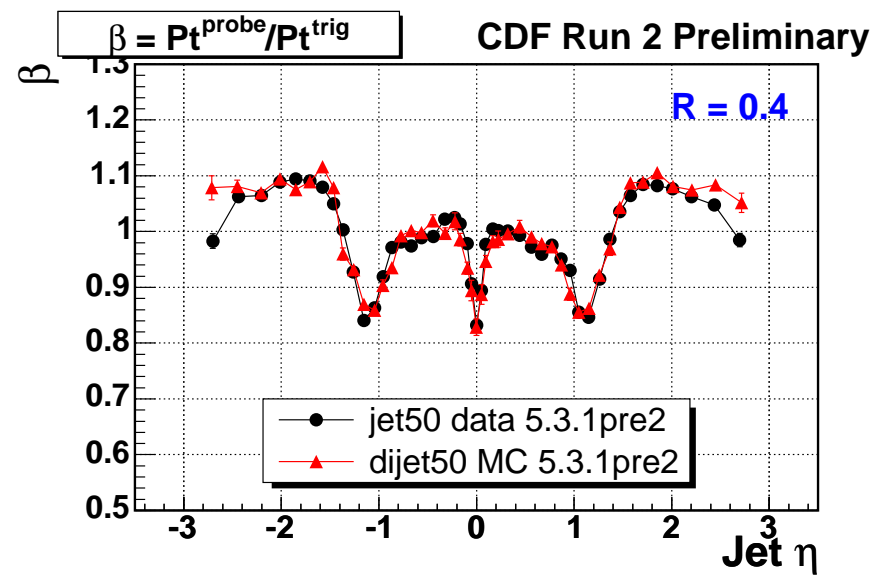

Figure 4.3: Dijet balance studies [58] using data and PYTHIA-Tune A MC. The results show the CDF calorimeters layout with gaps at $\eta=0\left(\theta=90^{\circ}\right)$ and $\eta=1.2\left(\right.$ approximately $\left.\theta=30^{\circ}\right)$. 


\subsection{Trigger Study}

\subsubsection{Jet Trigger datasets}

The trigger datasets used in this analysis are Single Tower 5 (ST5), Single Tower 10 (ST10), JET 20, JET 50, JET 70 and JET 100. The flow of the different datasets and the prescales are shown in figure 4.4. All the prescales for jet triggers are done by accepting a fixed fraction of events passing the corresponding trigger requirement.

- The Level 1 jet triggers use the L1 Calorimeter hardware. The two L1 jet triggers, ST5 and ST10, require energy thresholds to the calorimeter towers (EM + HAD) above $5 \mathrm{GeV}$ and $10 \mathrm{GeV}$, respectively. The ST5 includes a prescale factor of 20 or 50 (1 event accepted from each 20 or 50 events, depending on the run period), while ST10 is unprescaled.

- The Level 2 jet triggers are based on the L2 CAL hardware system. The clustering algorithm basically combines continuous calorimeter towers with an energy higher than a predetermined threshold. Each cluster starts with a tower above a "seed" threshold ( $3 \mathrm{GeV})$ and all towers above a second lower "shoulder" threshold $(1 \mathrm{GeV})$ that form a contiguous region with the seed tower are added to the cluster. The size of the cluster expands until no towers adjacent to the cluster have energy over the second threshold. An scheme of the cluster finder algorithm can be found in figure 4.5. The four trigger datasets are collected using cluster thresholds of 15, 40,60 and $90 \mathrm{GeV}$, respectively, and nominal prescale factors of 12 or 25 for JET 20, 1 or 5 for JET 50 and 8 for JET 70.

- The Level 3 jet triggers select events that have at least a jet with $E_{T}$ higher than a predetermined threshold. The jets are reconstructed using a jet cone algorithm (see section 2.8.2) with the parameter $\mathrm{R}$ set to 0.7 . The jet energy thresholds, as the dataset names indicate, are: $20 \mathrm{GeV}$ for JET 20, $50 \mathrm{GeV}$ for JET 50, $70 \mathrm{GeV}$ for JET 70 and $100 \mathrm{GeV}$ for JET 100. 


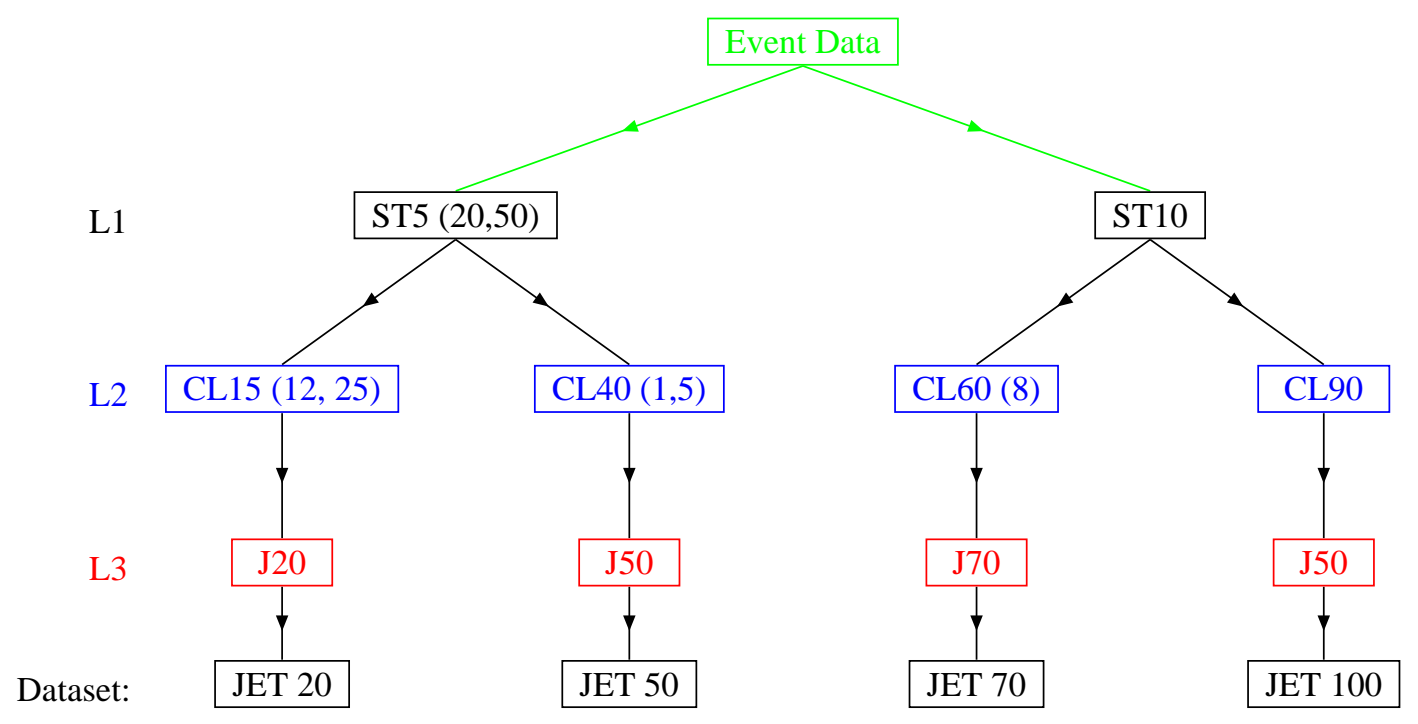

Figure 4.4: Trigger flow for the jet triggers. Typical prescales used during data taking are given in parentheses.
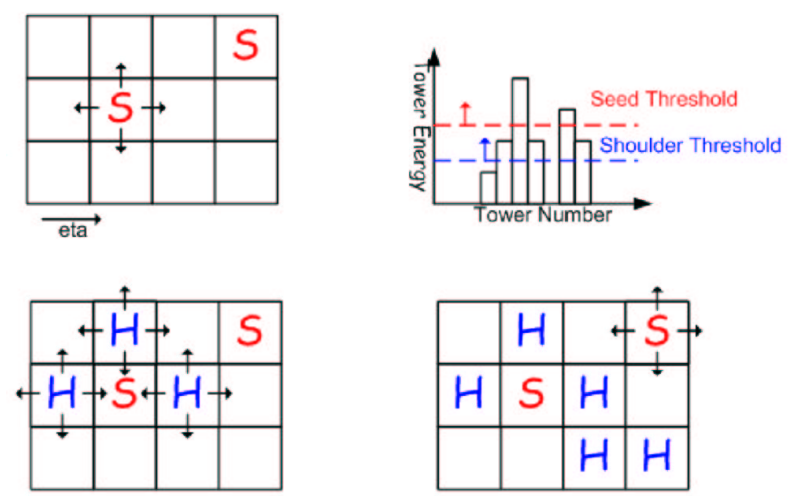

Figure 4.5: The cluster finder algorithm starts selecting all the trigger towers above a seed threshold called "seeds". The seed with lowest $\eta$ is chosen. In the case that two seed have the same $\eta$, the one with lowest $\phi$ is taken. The neighboring towers to the seed with an energy over a second threshold, shoulder towers, are added to the first one. The size of the cluster expands until no towers adjacent to the cluster have energy over the second threshold. The finder process is repeated with the next seed. 
The measurements are limited to jet transverse momenta for which the trigger is fully efficient. The trigger efficiencies at the different trigger levels (L1, L2 and L3) are determined using the trigger flow for the jet triggers (see figure 4.4), in such a way that to evaluate the efficiencies of a particular dataset, another dataset with lower energy thresholds is employed. Thus, the following scheme is used:

- get ST5 (L1) efficiency obtained from high $p_{T}$ muon dataset.

- get CL15 (L2) and J20 (L3) efficiencies obtained from ST5 dataset.

- get CL40 (L2), J50 (L3) and ST10 (L1) efficiencies obtained from JET 20 dataset.

- get CL60 (L2) and J70 (L3) efficiencies obtained from JET 50 dataset.

- get CL90 (L2) and J100 (L3) efficiencies obtained from JET 70 dataset.

The trigger efficiencies for each level are shown in figures 4.6 and 4.7. For each dataset and jet rapidity region, the global trigger efficiency is calculated by multiplying the corresponding L1, L2 and L3 trigger efficiencies, as it is illustrated in figure 4.8. The value for which the global trigger efficiency reaches $99 \%$ is initially considered. In order to avoid turn on trigger effects during the evaluation of the systematic uncertainties, due to the jet energy scale determination, the $p_{T, C A L}^{j e t}$ thresholds considered are increased by $5 \%$ with respect to the corresponding $99 \%$ trigger efficiency point. Table 4.1 summarizes the different thresholds for the different datasets and rapidity ranges.

\begin{tabular}{|c|c|c|}
\hline dataset & $99 \% p_{T, C A L}^{j e t}$ & $+5 \%$ of $99 \% p_{T, C A L}^{j e t}$ \\
\hline ST5 & $24 / 24 / 25 / 30 / 31$ & $26 / 26 / 27 / 32 / 33$ \\
\hline JET 20 & $30 / 30 / 31 / 32 / 31$ & $32 / 32 / 33 / 34 / 33$ \\
\hline JET 50 & $57 / 57 / 61 / 68 / 70$ & $60 / 60 / 65 / 72 / 74$ \\
\hline JET 70 & $77 / 80 / 86 / 92 / 96$ & $81 / 80 / 91 / 97 / 101$ \\
\hline JET 100 & $111 / 113 / 118 / 131 / 133$ & $117 / 119 / 124 / 138 / 140$ \\
\hline
\end{tabular}

Table 4.1: Obtained $p_{T, C A L}^{\text {jet }}$ thresholds $[\mathrm{GeV} / \mathrm{c}]$ for the different datasets and for different rapidity regions: $\left|y^{\text {jet }}\right|<$ $0.1 / 0.1<\left|y^{\text {jet }}\right|<0.7 / 0.7<\left|y^{\text {jet }}\right|<1.1 / 1.1<\left|y^{\text {jet }}\right|<1.6 / 1.6<\left|y^{\text {jet }}\right|<2.1$. The 99\% global trigger efficiency thresholds are reported in the second column. The $5 \%$ increased thresholds are reported in the third column. 

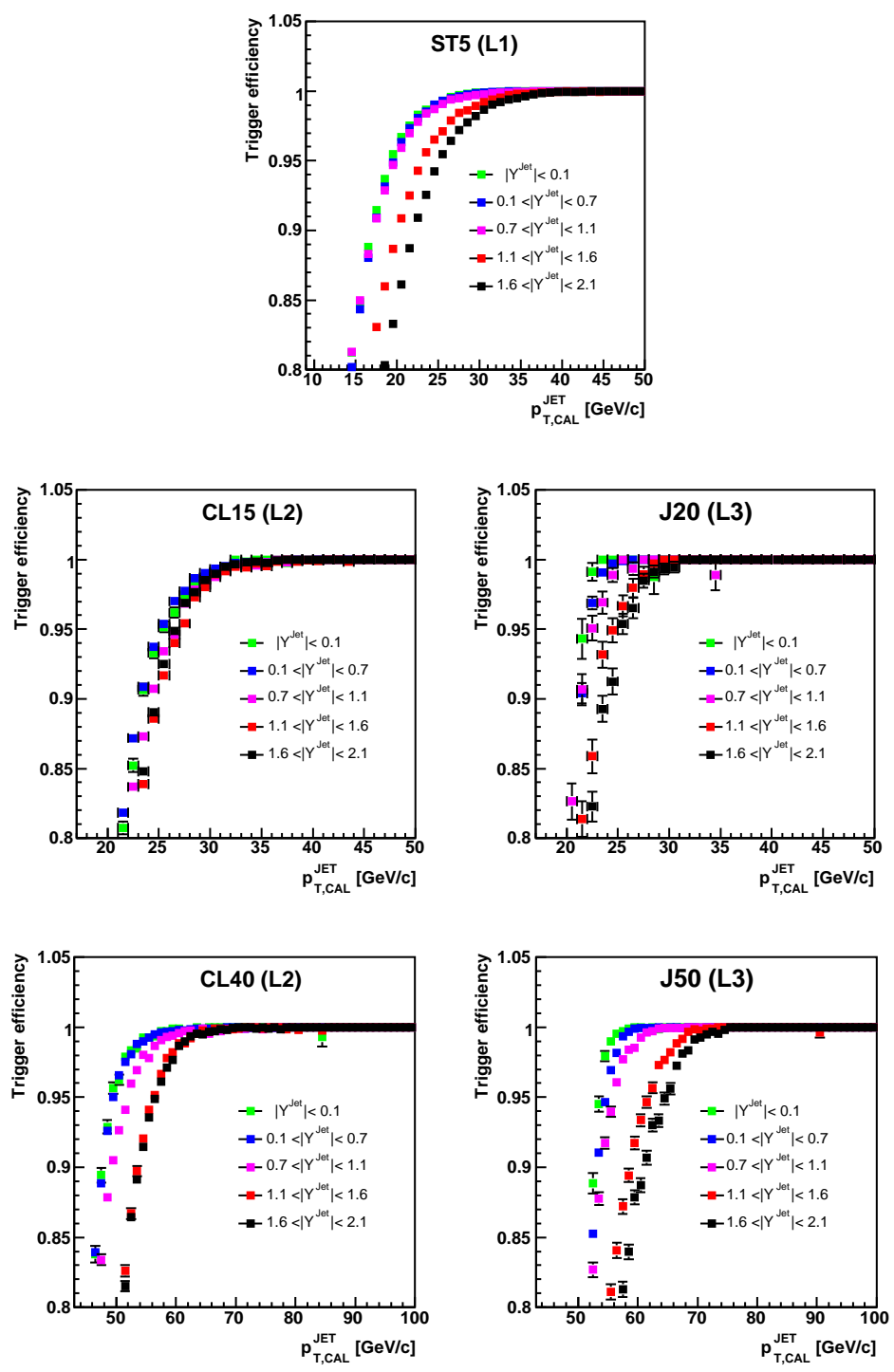

Figure 4.6: Trigger efficiency versus $p_{T, C A L}^{\text {jet }}$ for ST5 (L1 of ST5, JET 20 and JET 50 datasets) obtained using muon dataset (top), for CL15 (L2 of JET 20 dataset) obtained using ST5 dataset (center left), for J20 (L3 of JET 20 dataset) obtained using ST5 dataset (center right), for CL40 (L2 of JET 50 dataset) obtained using JET 20 dataset (bottom left) and for J50 (L3 of JET 50 dataset) obtained using JET 20 dataset (bottom right). 

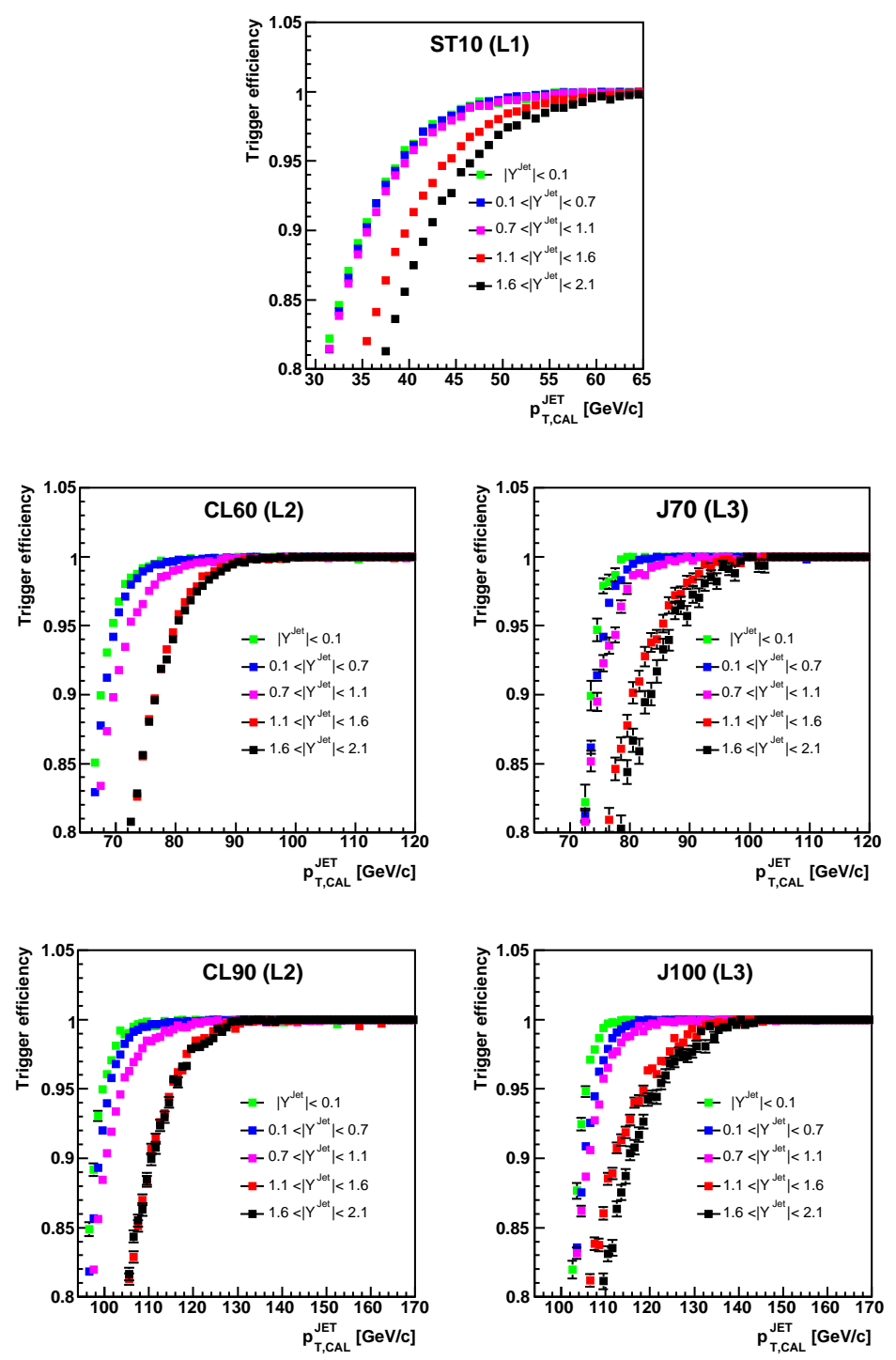

Figure 4.7: Trigger efficiency versus $p_{T, C A L}^{\text {jet }}$ for ST10 (L1 of JET 70 and JET 100 datasets) obtained using JET 20 dataset (top), for CL60 (L2 of JET 70 dataset) obtained using JET 50 dataset (center left), for J70 (L3 of JET 70 dataset) obtained using JET 50 dataset (center right), for CL90 (L2 of JET 100 dataset) obtained using JET 70 dataset (bottom left) and for J100 (L3 of JET 100) obtained using JET 70 dataset (bottom right). 


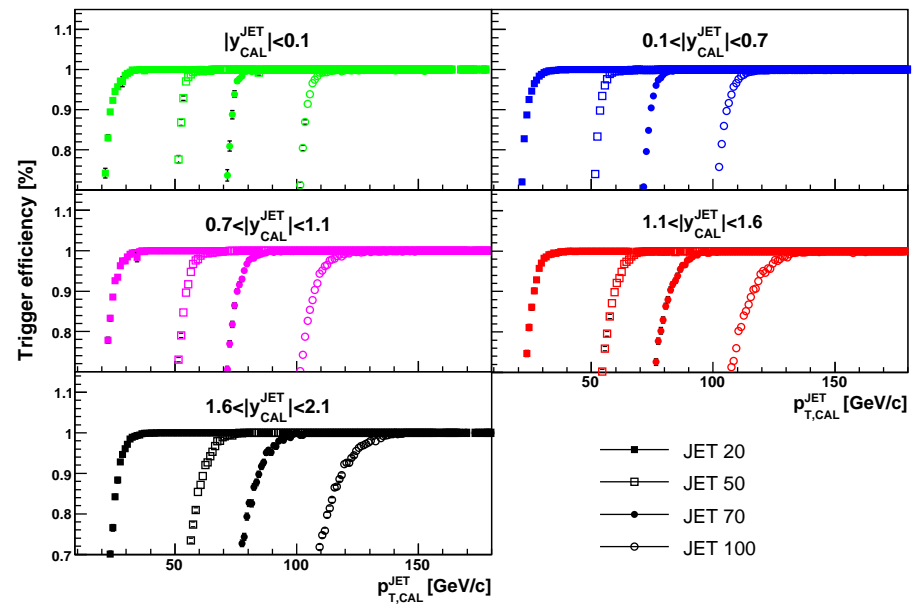

Figure 4.8: Combined trigger efficiency $(\mathrm{L} 1 \times \mathrm{L} 2 \times \mathrm{L} 3)$ versus $p_{T, C A L}^{\text {jet }}$ for JET 20, JET 50, JET 70 and JET 100 for jets in the different $\left|y^{j e t}\right|$ regions.

These trigger efficiency thresholds fix the $p_{T}$ bin range where each dataset is used. Figure 4.9 illustrates the measured $p_{T, C A L}^{j e t}$ distribution in the five rapidity regions after combining the different datasets. In table 4.2 the dataset used in each bin of rapidity and $p_{T, C A L}^{j e t}$ are listed in detail.

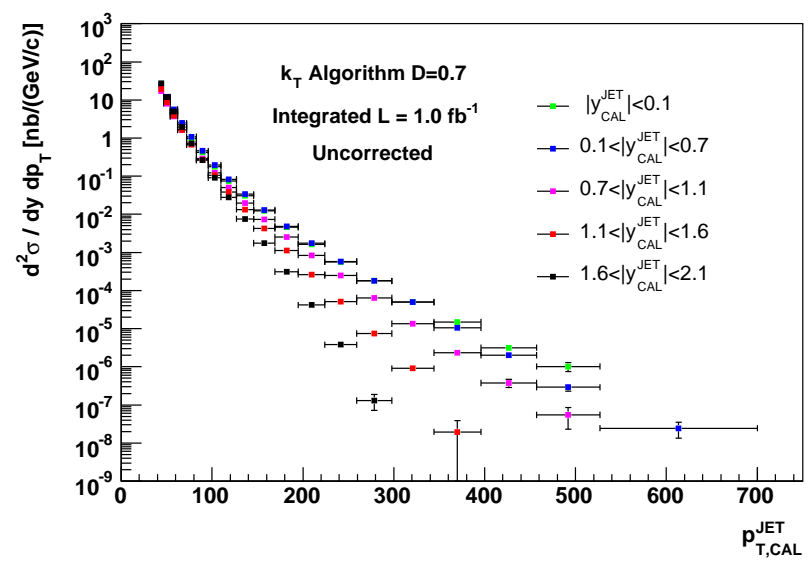

Figure 4.9: Measured $p_{T, C A L}^{\text {jet }}$ distribution in the different rapidity regions up to $\left|y^{\text {jet }}\right|=2.1$. 


\begin{tabular}{|c|c|c|c|c|c|}
\hline$p_{T, C A L}^{\text {jet }}$ & $\left|y^{\text {jet }}\right|<0.1$ & $0.1<\left|y^{\text {jet }}\right|<0.7$ & $0.7<\left|y^{\text {jet }}\right|<1.1$ & $1.1<\left|y^{\text {jet }}\right|<1.6$ & $1.6<\left|y^{\text {jet }}\right|<2.1$ \\
\hline $35-41$ & JET 20 & JET 20 & JET 20 & JET 20 & JET 20 \\
\hline $41-47$ & JET 20 & JET 20 & JET 20 & JET 20 & JET 20 \\
\hline $47-54$ & JET 20 & JET 20 & JET 20 & JET 20 & JET 20 \\
\hline $54-62$ & JET 20 & JET 20 & JET 20 & JET 20 & JET 20 \\
\hline $62-72$ & JET 50 & JET 50 & JET 20 & JET 20 & JET 20 \\
\hline $72-83$ & JET 50 & JET 50 & JET 50 & JET 50 & JET 20 \\
\hline $83-96$ & JET 70 & JET 50 & JET 50 & JET 50 & JET 50 \\
\hline $96-110$ & JET 70 & JET 70 & JET 70 & JET 50 & JET 50 \\
\hline $110-127$ & JET 70 & JET 70 & JET 70 & JET 70 & JET 70 \\
\hline $127-146$ & JET 100 & JET 100 & JET 100 & JET 70 & JET 70 \\
\hline $146-169$ & JET 100 & JET 100 & JET 100 & JET 100 & JET 100 \\
\hline $169-195$ & JET 100 & JET 100 & JET 100 & JET 100 & JET 100 \\
\hline $195-224$ & JET 100 & JET 100 & JET 100 & JET 100 & JET 100 \\
\hline $224-259$ & JET 100 & JET 100 & JET 100 & JET 100 & JET 100 \\
\hline $259-298$ & JET 100 & JET 100 & JET 100 & JET 100 & JET 100 \\
\hline $298-344$ & JET 100 & JET 100 & JET 100 & JET 100 & JET 100 \\
\hline $344-396$ & JET 100 & JET 100 & JET 100 & JET 100 & JET 100 \\
\hline $396-457$ & JET 100 & JET 100 & JET 100 & JET 100 & JET 100 \\
\hline $457-527$ & JET 100 & JET 100 & JET 100 & JET 100 & JET 100 \\
\hline $527-700$ & JET 100 & JET 100 & JET 100 & JET 100 & JET 100 \\
\hline
\end{tabular}

Table 4.2: $p_{T}^{\text {jet }}$ bins and the corresponding datasets used for the measured $p_{T, C A L}^{\text {jet }}$ distribution in the different rapidity regions. 


\subsection{Pile-up Correction}

The measured $p_{T, C A L}^{j e t}$ includes contributions from multiple proton-antiproton interactions per bunch crossing. The data used in the measurement were collected with an average instantaneous luminosities of $41 \cdot 10^{30} \mathrm{~cm}^{-2} \mathrm{~s}^{-1}$, which corresponds to an average of 1.5 inelastic $p \bar{p}$ interaction per bunch crossing. At the highest instantaneous luminosity the average is 5.9 interactions per bunch crossing.

In CDF, these multiple interactions are identified by the presence of additional primary vertices. For each jet in the event, the measured $p_{T, C A L}^{j e t}$ is corrected for the effect of the pile-up by removing a certain amount of transverse momentum, $\varepsilon$, per each additional primary vertex in the event:

$$
p_{T, C A L}^{j e t}(\text { Pile-up Corrected })=p_{T, C A L}^{j e t}-\varepsilon \times(N V-1)
$$

where $N V$ is the number of primary vertices reconstructed in the event.

The quantity $\varepsilon$ is determined from data for jets in the central calorimeter region by requiring that the ratio of cross sections at low and high instantaneous luminosities does not show any $p_{T, C A L}^{\text {jet }}$ dependence after applying the pile-up correction. The low instantaneous luminosity subsample corresponds to instantaneous luminosities between 5 and $15 \cdot 10^{30} \mathrm{~cm}^{-2} \mathrm{~s}^{-1}$, while the high instantaneous luminosity sample corresponds to instantaneous luminosities $>35 \cdot 10^{30} \mathrm{~cm}^{-2} \mathrm{~s}^{-1}$. In all the cases, the obtained ratio is fitted by a constant. Then the evolution of the $\chi^{2}$ of the fits is drawn as a function of $\varepsilon$. The $\varepsilon$ value that gives the minimum $\chi^{2}$ is chosen as the nominal correction. Going to lower (higher) values of $\varepsilon$, the first correction for which the $\chi^{2}$ has increased by more than 9 units, $3 \sigma$, is taken as the low (high) limit on $\varepsilon$ and will be considered as systematic uncertainties on this correction. Figures 4.10 and 4.11 show the ratios as a function of $p_{T, C A L}^{\text {jet }}$ for different values of $\varepsilon$ and the evolution of the $\chi^{2}$ of the fits. The normal value for $\varepsilon$ was found to be $1.86 \pm 0.23 \mathrm{GeV} / \mathrm{c}$.

Similar studies have been carried out for jets in the different rapidities. In all cases, the ratio does not show any $p_{T, C A L}^{\text {jet }}$ dependence when $\varepsilon=1.86 \mathrm{GeV} / \mathrm{c}$ is applied as is shown in figure 4.12. It is important to mention that additional corrections are included in $\varepsilon$ (see section 4.4.3) to account for the different response in different rapidity regions in the calorimeter. Table 4.3 summarizes the obtained values of $\varepsilon$ together with the uncertainties for the different rapidity regions. 

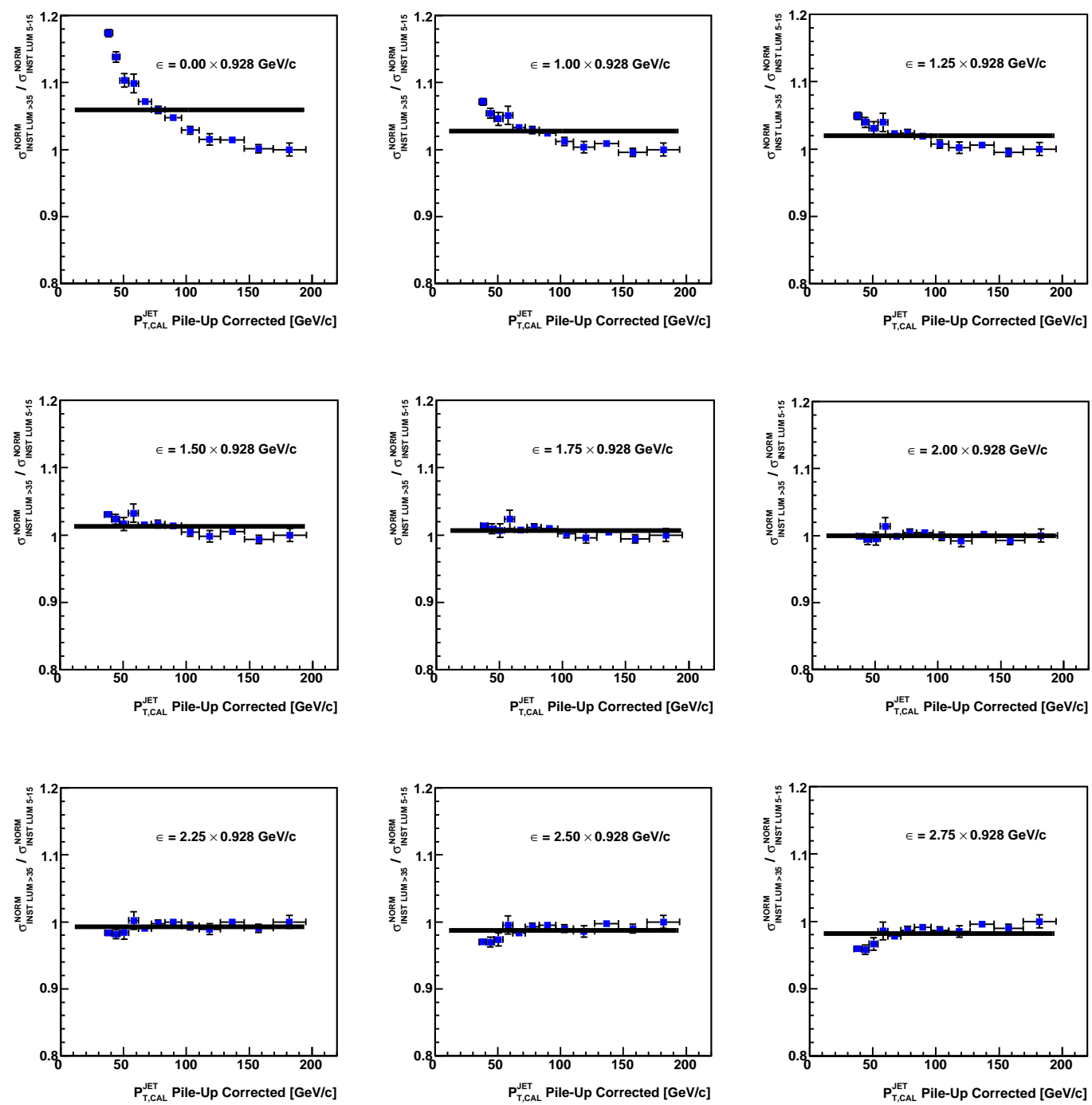

Figure 4.10: Ratio of cross sections for different pile-up corrections in two bins of instantaneous luminosity, $>35$. $10^{30} \mathrm{~cm}^{-2} \mathrm{~s}^{-1}$ and 5 to $15 \cdot 10^{30} \mathrm{~cm}^{-2} \mathrm{~s}^{-1}$, for jets in the region $0.1<\left|y^{j e t}\right|<0.7$. A fit to a constant is superimposed to the data. The $\varepsilon$ values are expressed as factors of $0.928 \mathrm{GeV} / \mathrm{c}$ that is the value used in studies with the MidPoint algorithm. It was obtained as the average $p_{T}$ deposited by minbias events in cones of size 0.7 randomly drawn in the central calorimeter [58]. 

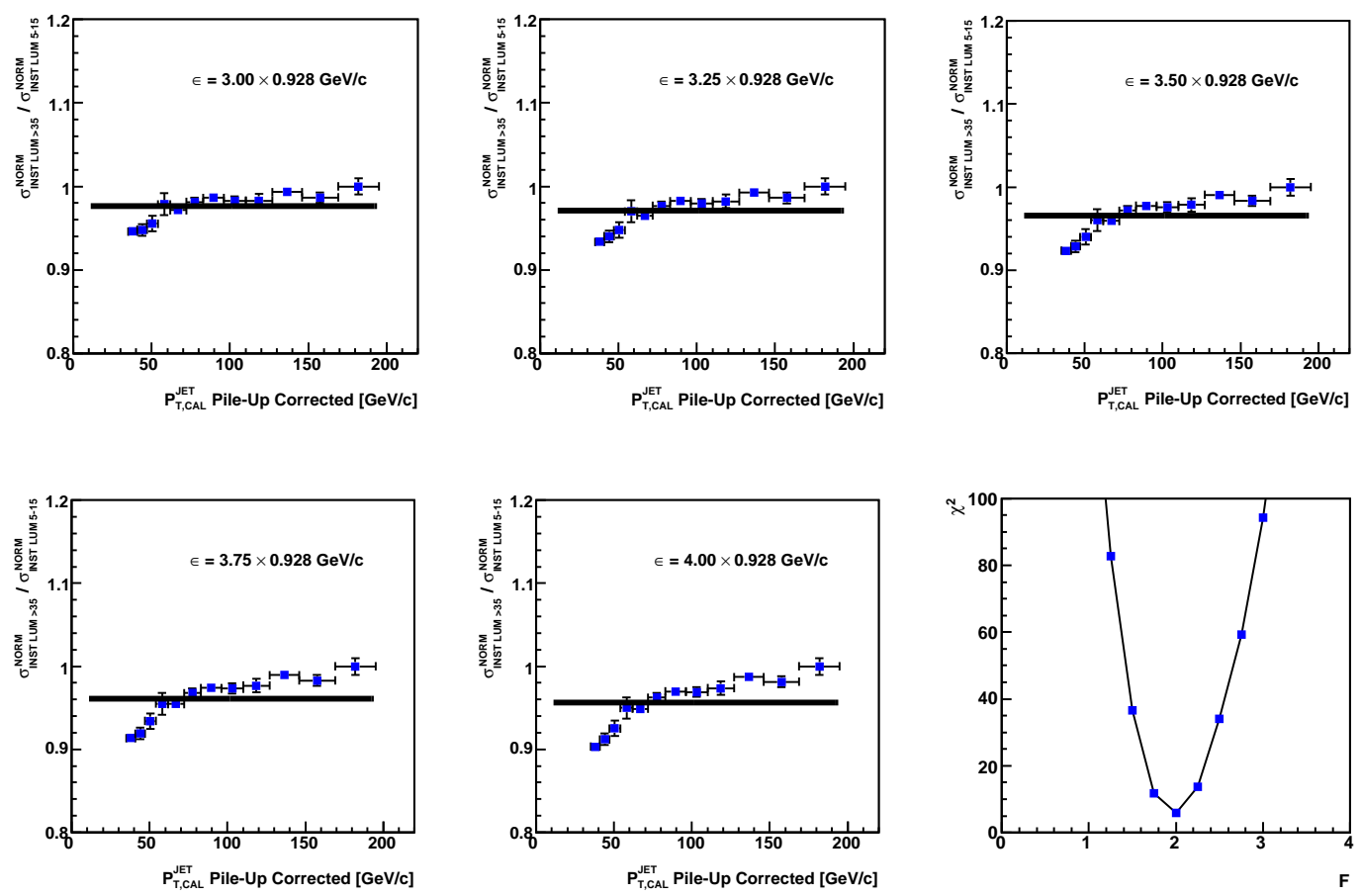

Figure 4.11: Ratio of cross sections for different pile-up corrections in two bins of instantaneous luminosity, $>35$. $10^{30} \mathrm{~cm}^{-2} \mathrm{~s}^{-1}$ and 5 to $15 \cdot 10^{30} \mathrm{~cm}^{-2} \mathrm{~s}^{-1}$, for jets in the region $0.1<\left|y^{j e t}\right|<0.7$. A fit to a constant is superimposed to the data. The $\varepsilon$ values are expressed as factors of $0.928 \mathrm{GeV} / \mathrm{c}$ that is the value used in studies with the MidPoint algorithm. It was obtained as the average $p_{T}$ deposited by minbias events in cones of size 0.7 randomly drawn in the central calorimeter [58]. The bottom right plot is the evolution of the $\chi^{2}$ of the fits is drawn as a function of $\mathrm{F}$ $(\varepsilon=\mathrm{F} \times 0.928 \mathrm{GeV} / \mathrm{c})$. 


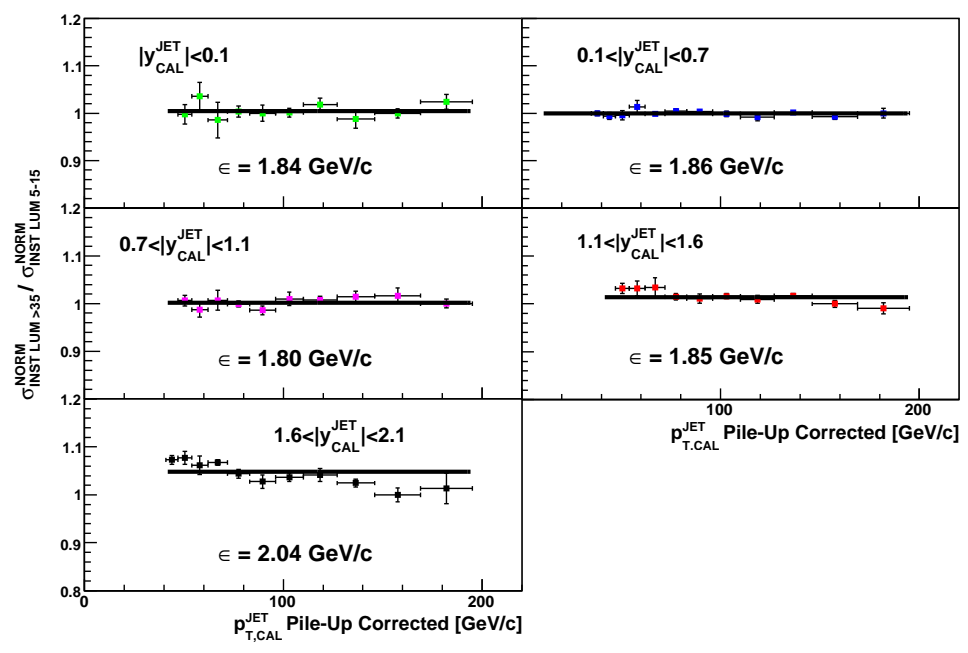

Figure 4.12: Ratio of cross sections in two bins of instantaneous luminosity, when the pile-up corrections are applied, for jets in the different $\left|y^{j e t}\right|$ regions.

\begin{tabular}{|c|c|}
\hline Rapidity region & $\varepsilon$ \\
\hline$\left|y^{\text {jet }}\right|<0.1$ & $1.84 \pm 0.23 \mathrm{GeV} / \mathrm{c}$ \\
\hline $0.1<\left|y^{\text {jet }}\right|<0.7$ & $1.86 \pm 0.23 \mathrm{GeV} / \mathrm{c}$ \\
\hline $0.7<\left|y^{\text {jet }}\right|<1.1$ & $1.80 \pm 0.23 \mathrm{GeV} / \mathrm{c}$ \\
\hline $1.1<\left|y^{\text {jet }}\right|<1.6$ & $1.85 \pm 0.23 \mathrm{GeV} / \mathrm{c}$ \\
\hline $1.6<\left|y^{\text {jet }}\right|<2.1$ & $2.04 \pm 0.26 \mathrm{GeV} / \mathrm{c}$ \\
\hline
\end{tabular}

Table 4.3: $\varepsilon$ values and their uncertainties for each rapidity region. 


\subsection{MC studies}

$\mathrm{MC}$ event samples are used to determine the corrections factors to unfold the measurements back to the hadron level. The MC samples have been generated using PYTHIA 6.203, with the Tune A, and HERWIG 6.4 MC generators. In both programs, the partonic interactions are generated using leading-order QCD matrix element, including initial and final-states parton showers. CTEQ5L parton distribution functions are used for the proton and antiproton. The generated samples are passed through the CDF detector simulation package. Different MC samples with different thresholds on $\hat{p}_{T}$ were produced to ensure enough MC statistics in all the $p_{T}^{\text {jet }}$ regions. Special care was taken to avoid any bias coming from the presence of the different $\hat{p}_{T}$ thresholds in the MC.

Prior to unfold the data with the MC samples (see section 4.5), several studies have been carried out to examine carefully the calorimeter response and jet reconstruction in the simulation.

\subsubsection{Raw variables comparison}

First, the agreement between data and MC has been checked on the quantities most relevant for this analysis. These variable are: the $\mathrm{Z}$ position of the primary vertex, the missing $E_{T}$ significance, the jet $\phi, y$ and $p_{T}$ distributions and the number of towers inside the jet.

The comparison of the different variables, shown in figures 4.13 to 4.17 , indicates that the CDF simulation gives a reasonable description of the data for the jet rapidities considered. However, the comparison of the multiplicity of the towers inside the jet for different rapidity regions indicates that the MC tends to underestimate the number of towers associated to the jet. This is mainly due to a non adequate simulation of the energy deposition in the calorimeter, in particular in the plug regions. Nevertheless, it should be mentioned that the multiplicity of the towers inside the jet is very sensitive to towers with very small energy $(\sim 100 \mathrm{MeV})$. 

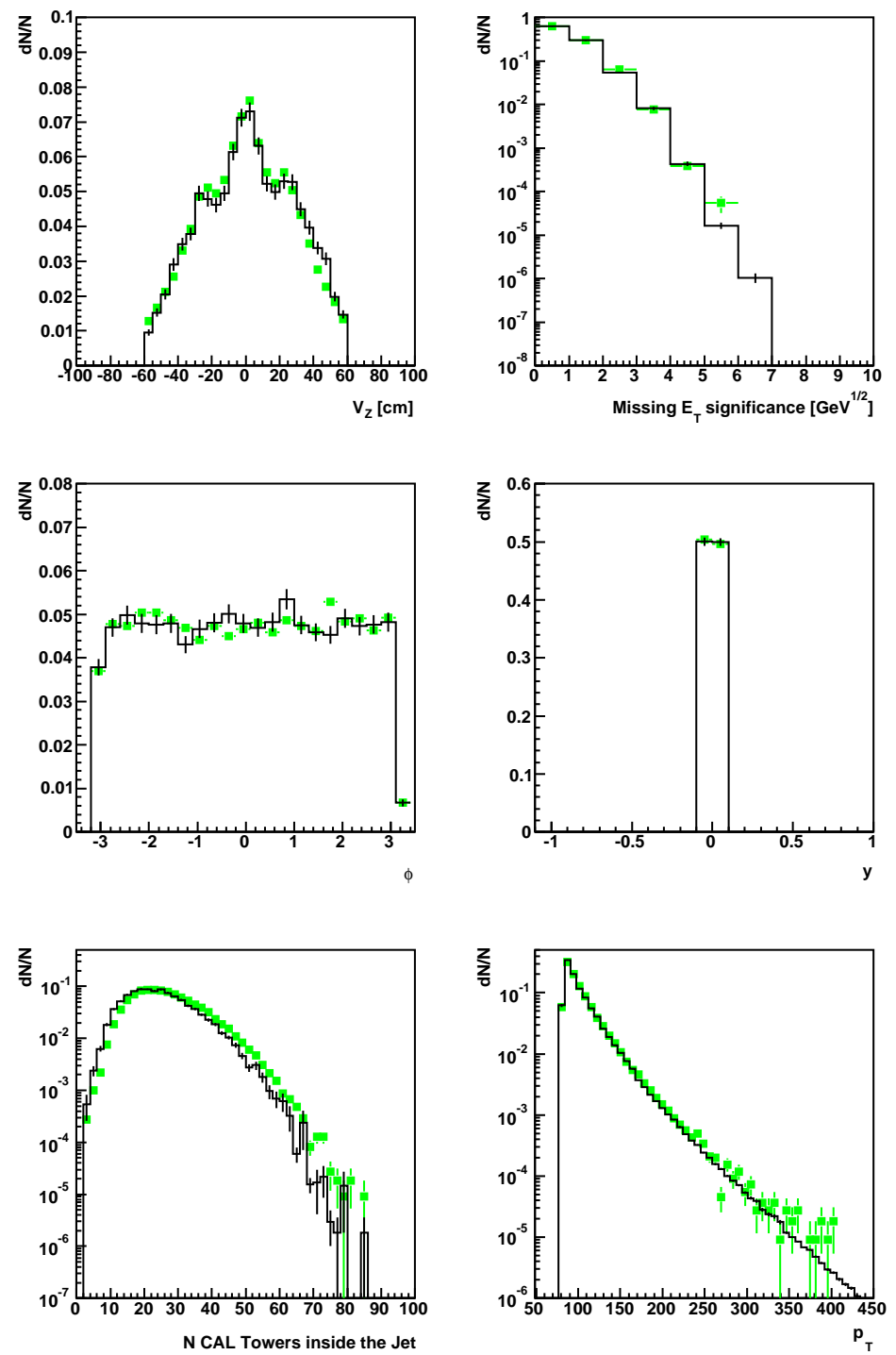

Figure 4.13: Distribution of the $\mathrm{Z}$ position of the primary vertex (top left), missing $E_{T}$ significance (top right), $\phi^{j e t}$ (center left), $y^{\text {jet }}$ (center right), number of calorimeter towers inside the jet distribution (bottom left) and $p_{T}$ (bottom right) for data (squares) and the corresponding MC samples (histogram) for jets in the rapidity region $\left|y^{\text {jet }}\right|<0.1$ with $p_{T, C A L}^{j e t}>83 \mathrm{GeV} / \mathrm{c}$. Only events with exactly 1 primary vertex are considered. 

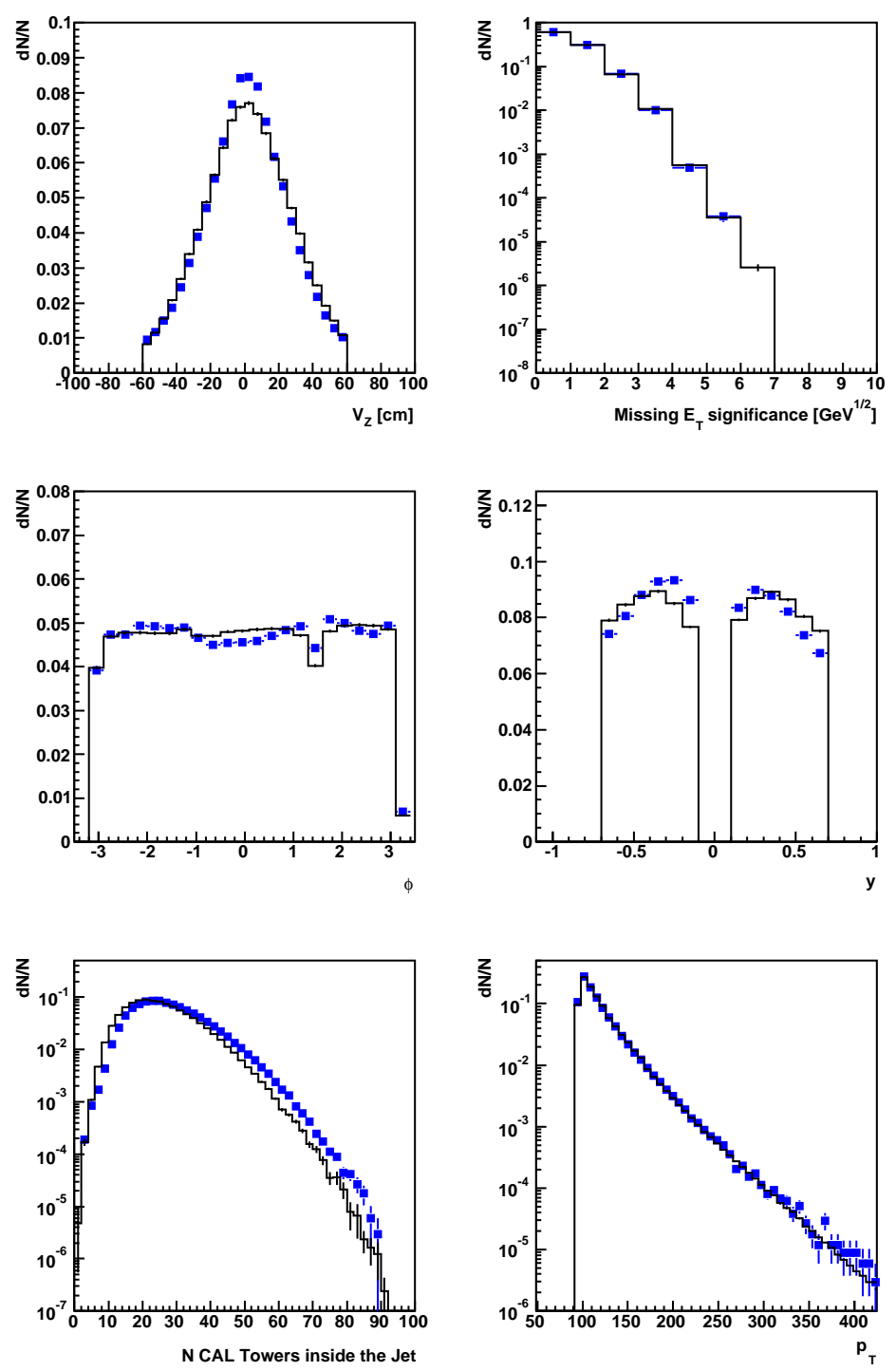

Figure 4.14: Distribution of the $\mathrm{Z}$ position of the primary vertex (top left), missing $E_{T}$ significance (top right), $\phi^{j e t}$ (center left), $y^{\text {jet }}$ (center right), number of calorimeter towers inside the jet distribution (bottom left) and $p_{T}$ (bottom right) for data (squares) and the corresponding MC samples (histogram) for jets in the rapidity region $0.1<\left|y^{\text {jet }}\right|<0.7$ with $p_{T, C A L}^{\text {jet }}>96 \mathrm{GeV} / \mathrm{c}$. Only events with exactly 1 primary vertex are considered. 

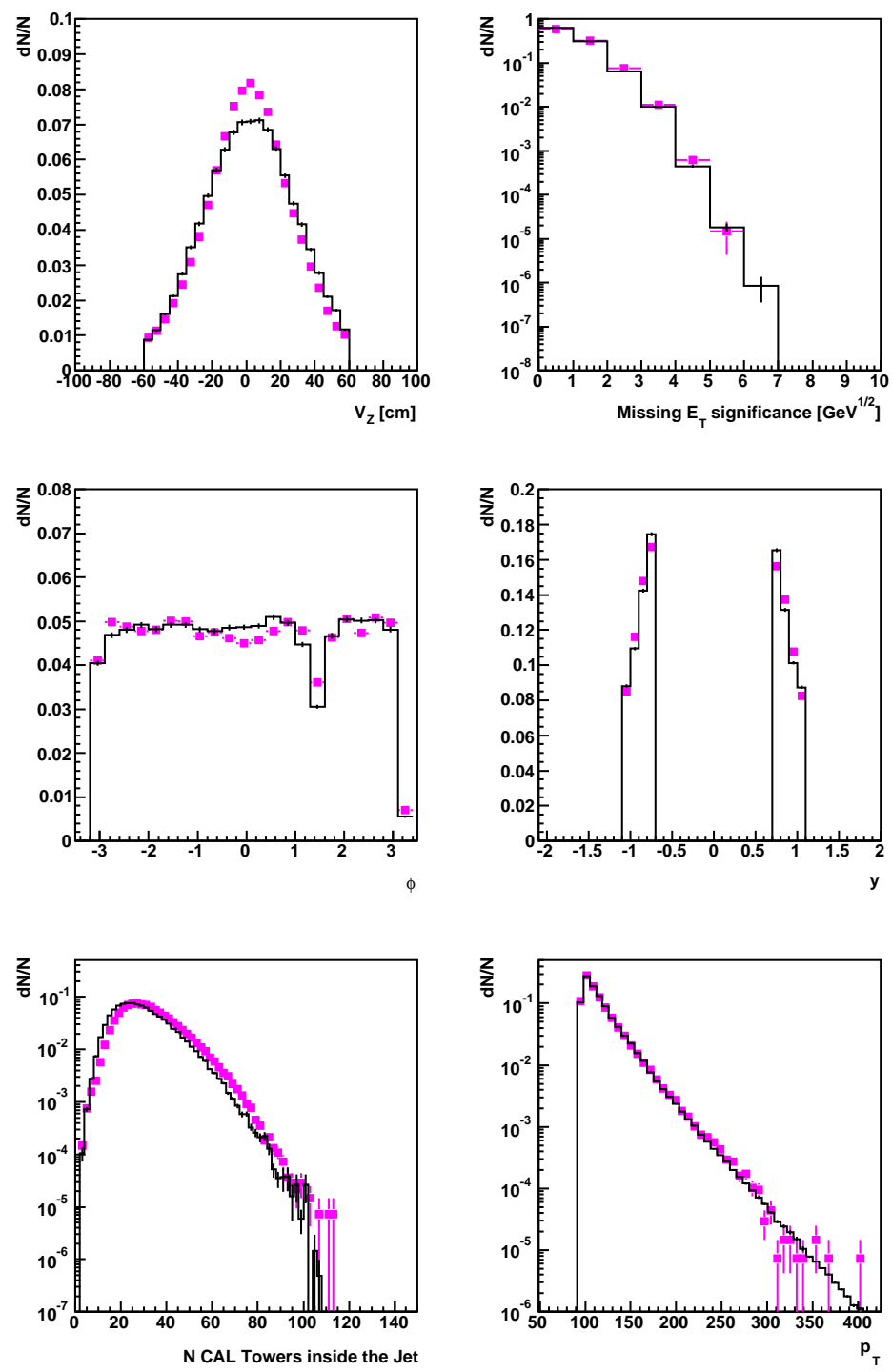

Figure 4.15: Distribution of the $\mathrm{Z}$ position of the primary vertex (top left), missing $E_{T}$ significance (top right), $\phi^{j e t}$ (center left), $y^{\text {jet }}$ (center right), number of calorimeter towers inside the jet distribution (bottom left) and $p_{T}$ (bottom right) for data (squares) and the corresponding MC samples (histogram) for jets in the rapidity region $0.7<\left|y^{\text {jet }}\right|<1.1$ with $p_{T, C A L}^{\text {jet }}>96 \mathrm{GeV} / \mathrm{c}$. Only events with exactly 1 primary vertex are considered. 

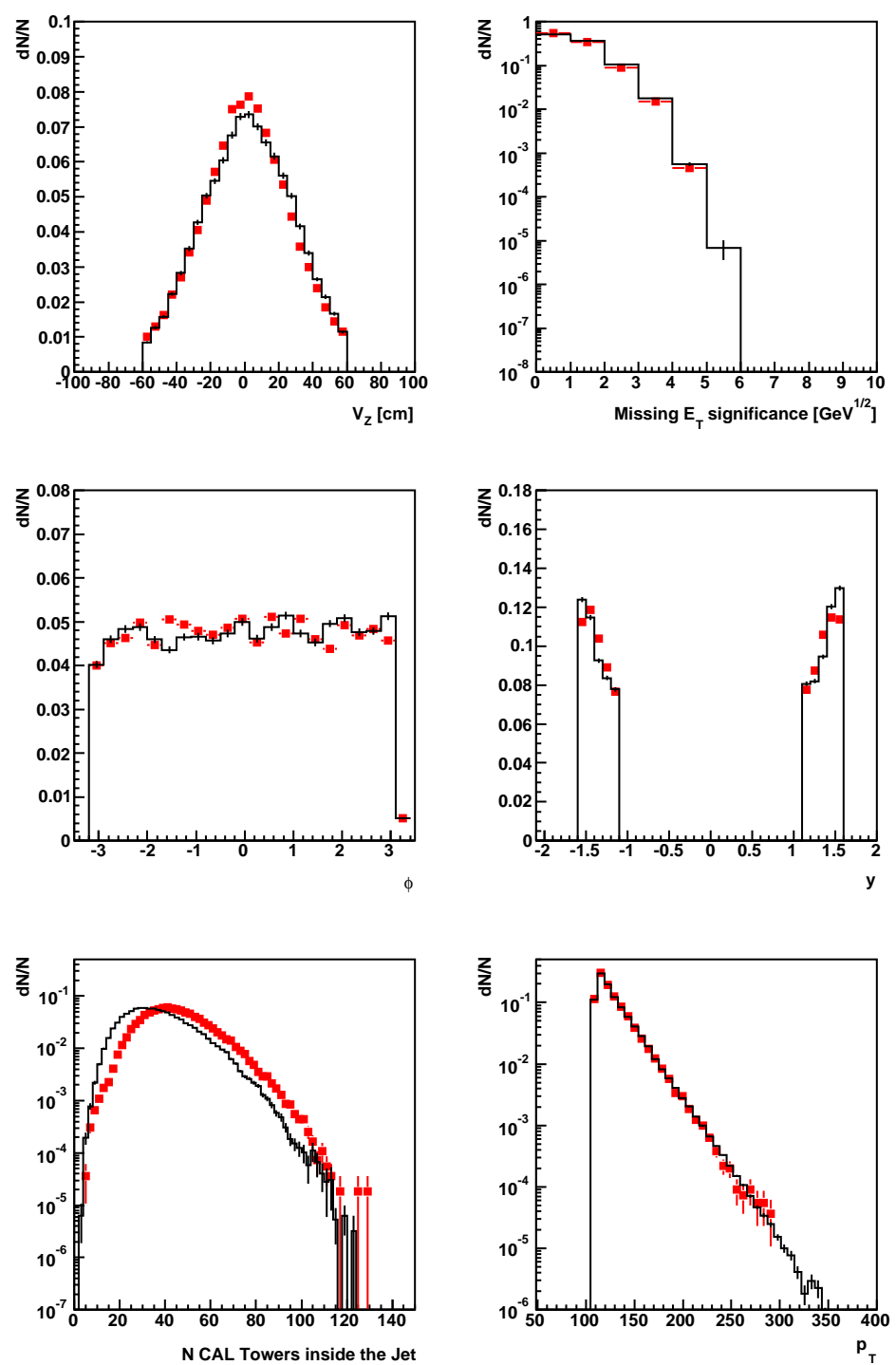

Figure 4.16: Distribution of the $\mathrm{Z}$ position of the primary vertex (top left), missing $E_{T}$ significance (top right), $\phi^{j e t}$ (center left), $y^{\text {jet }}$ (center right), number of calorimeter towers inside the jet distribution (bottom left) and $p_{T}$ (bottom right) for data (squares) and the corresponding MC samples (histogram) for jets in the rapidity region $1.1<\left|y^{\text {jet }}\right|<1.6$ with $p_{T, C A L}^{j e t}>110 \mathrm{GeV} / \mathrm{c}$. Only events with exactly 1 primary vertex are considered. 

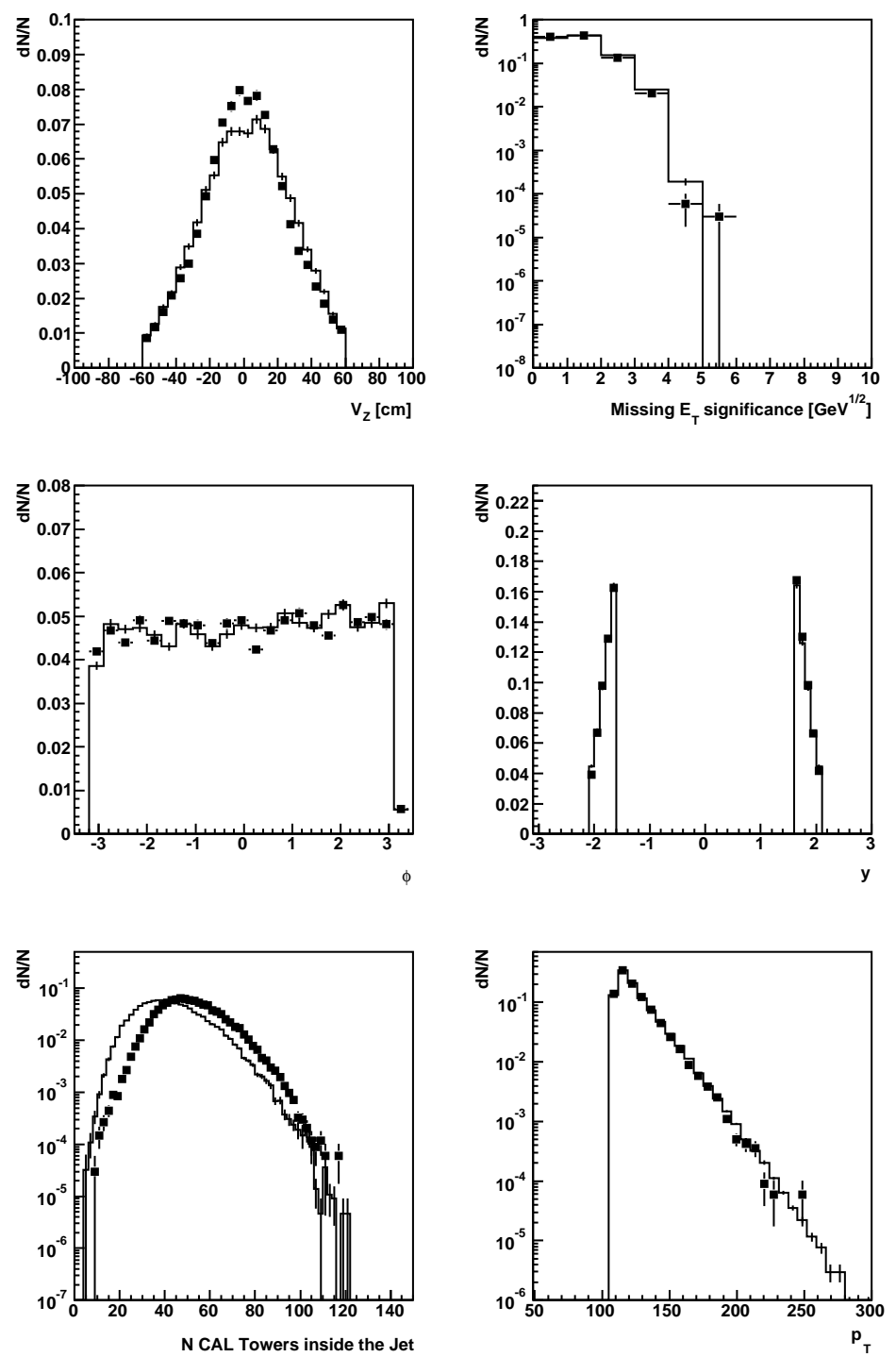

Figure 4.17: Distribution of the $\mathrm{Z}$ position of the primary vertex (top left), missing $E_{T}$ significance (top right), $\phi^{j e t}$ (center left), $y^{\text {jet }}$ (center right), number of calorimeter towers inside the jet distribution (bottom left) and $p_{T}$ (bottom right) for data (squares) and the corresponding MC samples (histogram) for jets in the rapidity region $1.6<\left|y^{\text {jet }}\right|<2.1$ with $p_{T, C A L}^{j e t}>110 \mathrm{GeV} / \mathrm{c}$. Only events with exactly 1 primary vertex are considered. 
As a result of these raw variable comparisons, more dedicated studies are necessary to test the accuracy of the $p_{T}^{\text {jet }}$ reconstruction in the MC. In next sections, the jet energy resolution is studied using the bisector method [59], while the dijet balance is employed to understand the energy scale with respect to the central calorimeter region, where it is known that the $\mathrm{MC}$ provides a proper description of the data [58].

\subsubsection{Bisector Method}

The $p_{T}$ unbalance between the two leading jets in dijet events is sensitive to gluon ratiation and detetor effects. The bisector method is based on the splitting of this $p_{T}$ unbalance in two components according to the definition of a bisector axis. The comparison of these two components provides an estimation of the jet energy resolution. The details of the method are explained below. The dijet events are selected according to the following criteria:

- 2 and only 2 jets with $p_{T, C A L}^{j e t}$ above $10 \mathrm{GeV} / \mathrm{c}$.

- One jets in the rapidity region $0.1<\left|y^{\text {jet }}\right|<0.7$ and the other jet in the rapidity region under study.

- 1 and only 1 primary vertex with $\left|V_{Z}\right| \leq 60 \mathrm{~cm}$.

- Missing $E_{T}$ significance criteria (see section 4.1.2).

The bisector axis is defined as the axis corresponding to the direction of the bisector of the 2 jets in the transverse plane. The jet in the region $0.1<\left|y^{j e t}\right|<0.7$ is chosen to be jet 1 and the other jet is jet 2. In this context, the following quantities are then defined as it is shown in figure 4.18:

- $p_{T}^{R A W 1}=p_{T, C A L}^{\text {jet }}\left(\right.$ jet 1) and $p_{T}^{R A W 2}=p_{T, C A L}^{\text {jet }}($ jet 2).

- $p_{T}^{M E A N}=\left(p_{T}^{R A W 1}+p_{T}^{R A W 2}\right) / 2$, that is the average $p_{T, C A L}^{\text {jet }}$ of the dijet event.

- $\gamma=\left|\left(\phi^{J E T T^{1}}-\phi^{J E T}{ }^{2}\right) / 2\right|, \gamma$ is the angle between the jets and the bisector axis in the transverse plane.

- $\Delta p_{T}^{/ /}= \pm\left(p_{T}^{R A W 1}+p_{T}^{R A W 2}\right) \cos (\gamma), \Delta p_{T}^{/ /}$is the part of the $p_{T}$ unbalance that is parallel to the bisector axis in the transverse plane, the \pm factor in the $\Delta p_{T}^{/ /}$definition is randomly chosen to be either + or -. Due to this the distribution of $\Delta p_{T}^{/ /}$is symmetric around 0 . 
- $\Delta p_{T}^{P E R P}=\left(p_{T}^{R A W 1}-p_{T}^{R A W 2}\right) \sin (\gamma), \Delta p_{T}^{P E R P}$ is the part of the $p_{T}$ unbalance that is perpendicular to the bisector axis in the transverse plane.

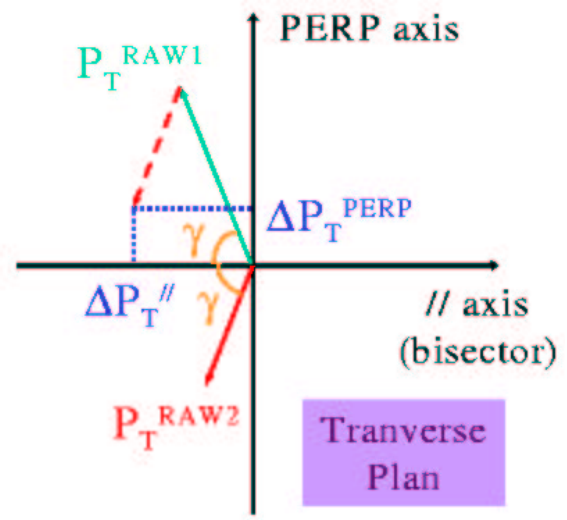

Figure 4.18: Illustration of the bisector method variables. The $p_{T}$ vectors of the two leading jets in the transverse plane are shown. $\gamma$ is the angle between the jets and the bisector axis. $\Delta p_{T}^{/ /}$and $\Delta p_{T}^{P E R P}$ are the part of the $p_{T}$ unbalance that are respectively parallel and perpendicular to the bisector axis.

The selected events are then separated in bins of the average $p_{T, C A L}^{j e t}$ of the dijet event. This $p_{T}$ binning is chosen to avoid bias in the mean and RMS values due to possible asymmetric distributions. For each bin, the following variables are calculated:

- $\sigma_{/ /}=$rms of the $\Delta p_{T}^{/ /}$distribution, resolution mainly sensitive to physics effects on the $p_{T}$ balance.

- $\sigma_{P E R P}=\mathrm{rms}$ of the $\Delta p_{T}^{P E R P}$ distribution, resolution sensitive to both detector and physics effects on the $p_{T}$ balance.

- $\sigma_{D}=\sqrt{\sigma_{P E R P}^{2}-\sigma_{/ /}^{2}}$, assuming physics effects are democratic in $\phi$ this term should represent the detector effects only.

Figures 4.19 to 4.24 show the values of $\sigma_{/ /}, \sigma_{P E R P}$ and $\sigma_{D}$ as a function of the average $p_{T, C A L}^{\text {jet }}$ of the dijet event for both data and $\mathrm{MC}$, as well as the ratios data/MC for these quantities in each rapidity region. As expected, the $\sigma_{/ /}$dependence on $p_{T}$ is much smaller than the $\sigma_{P E R P}$ one. The $\sigma_{/ /}$values obtained in the MC are about $20 \%$ lower than the ones observed on data. This effect may be linked to high order contributions not very well accounted for in the MC, which only 
includes leading-order matrix element plus parton shower. However, the crucial point here is the level of agreement between data and MC that is observed for $\sigma_{D}$ (figures 4.23 and 4.24).

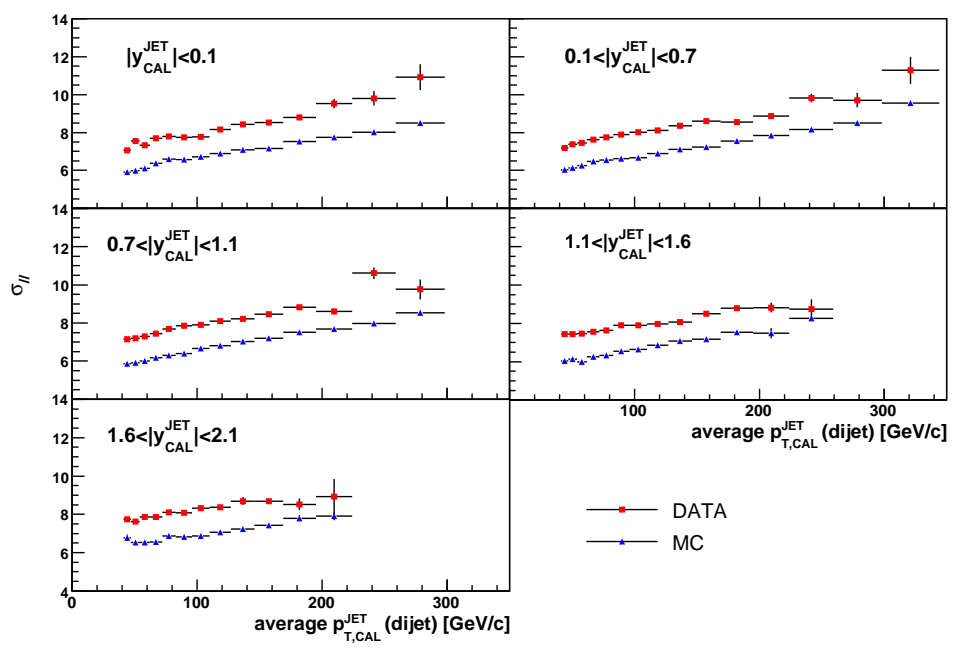

Figure 4.19: Bisector method study results: $\sigma_{/ /}$for DATA and MC for jets in different $\left|y^{j e t}\right|$ regions.

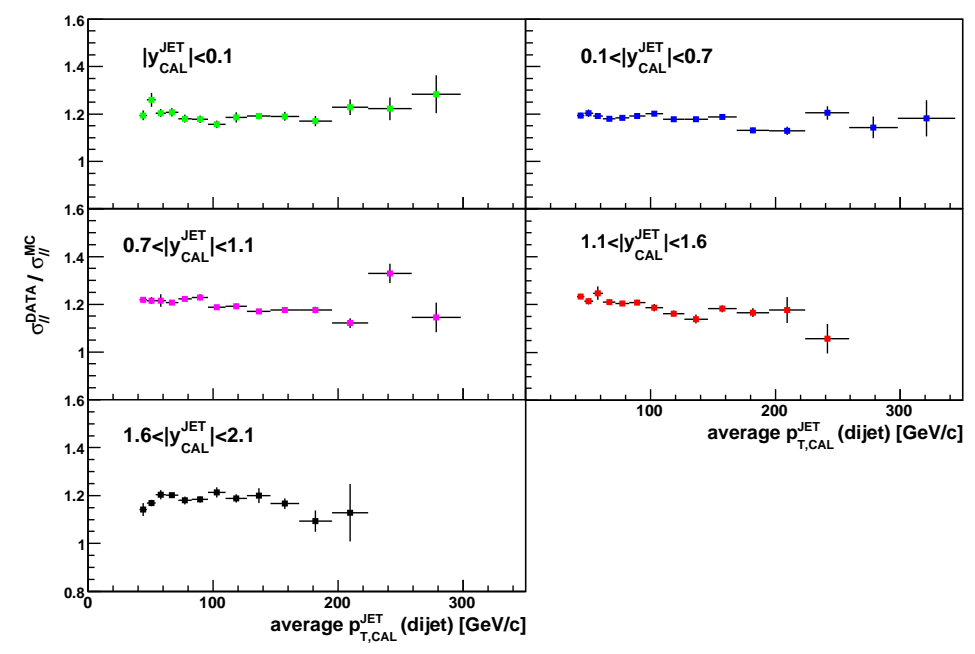

Figure 4.20: Bisector method study results: Ratio DATA / MC of the $\sigma_{/ /}$for jets in different $\left|y^{j e t}\right|$ regions. 


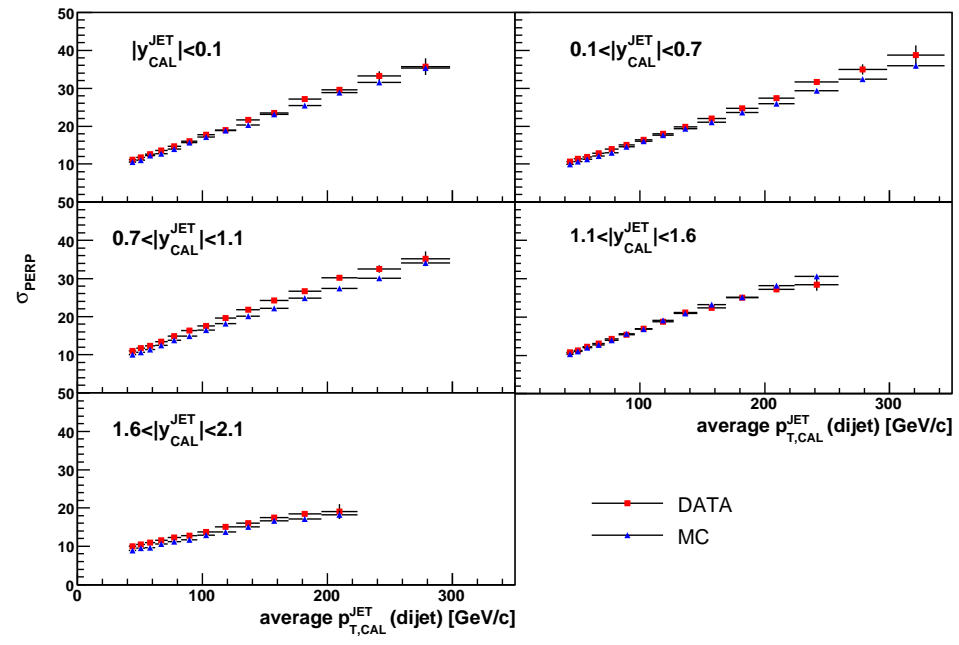

Figure 4.21: Bisector method study results: $\sigma_{P E R P}$ for DATA and MC for jets in different $\left|y^{\text {jet }}\right|$ regions.

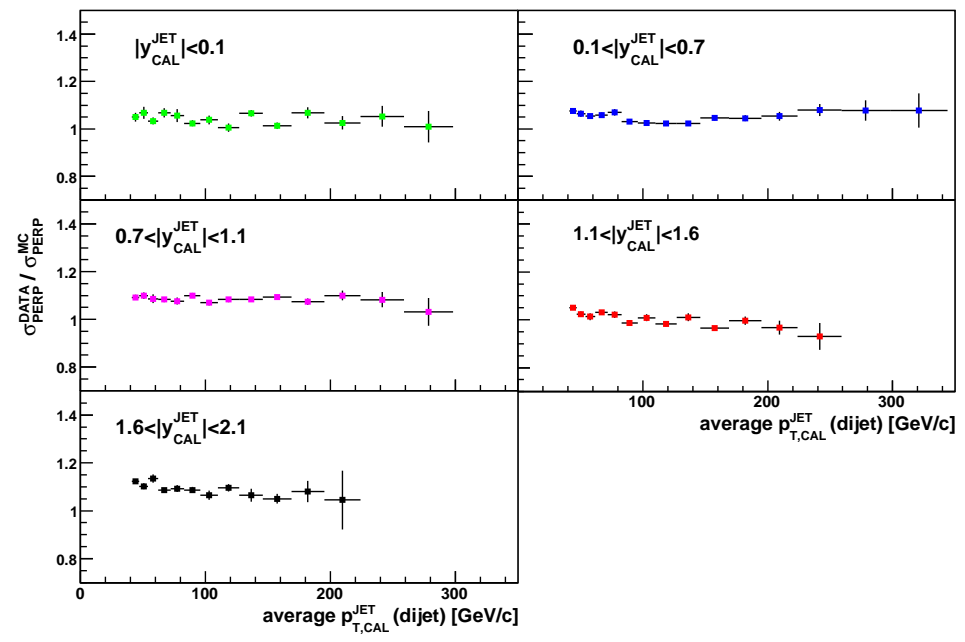

Figure 4.22: Bisector method study results: Ratio DATA / MC of the $\sigma_{P E R P}$ for jets in different $\left|y^{j e t}\right|$ regions. 


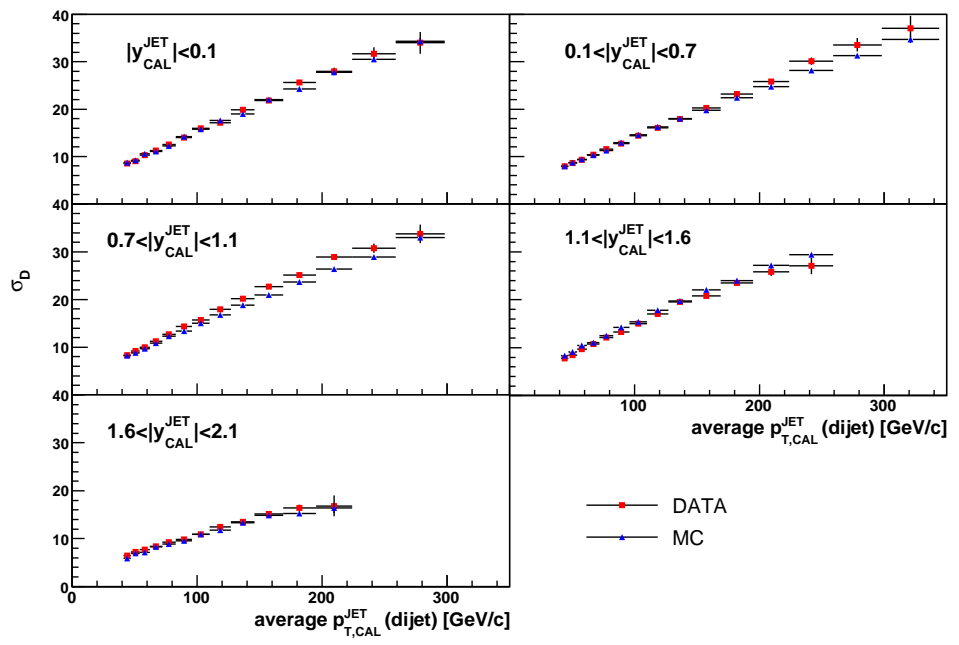

Figure 4.23: Bisector method study results: $\sigma_{D}$ for DATA and MC for jets in different $\left|y^{j e t}\right|$ regions.

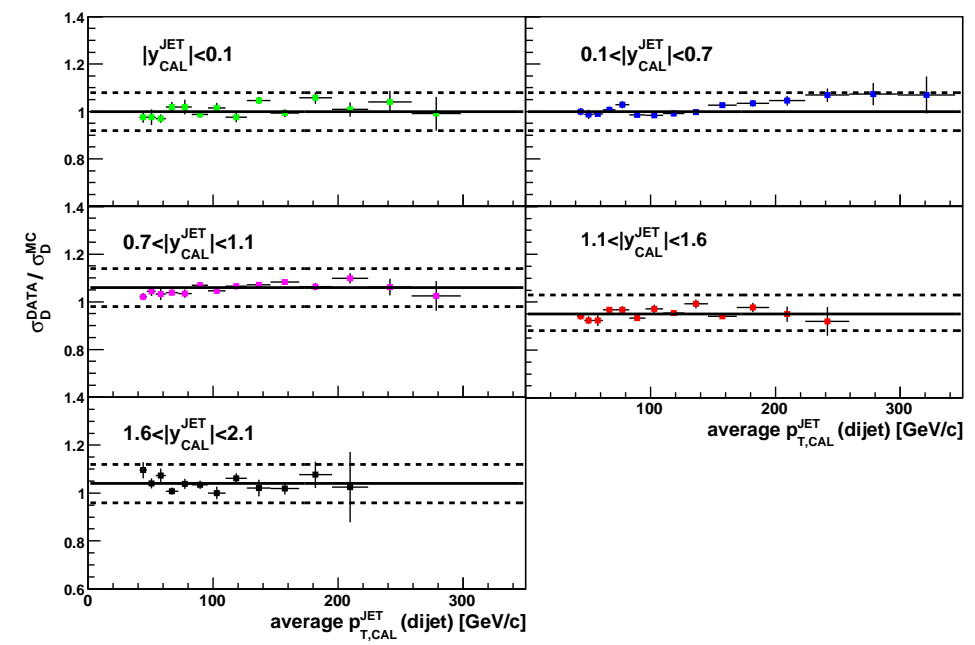

Figure 4.24: Bisector method study results: Ratio DATA / MC of the $\sigma_{D}$ for jets in different $\left|y^{\text {jet }}\right|$ regions. The solid line is the fit to a 0 order polynomial and the dashed lines show the $\pm 8 \%$ uncertainty considered. 
Figure 4.24 shows that for jets in the most central regions, $\left|y^{\text {jet }}\right|<0.1$ and $0.1<\left|y^{\text {jet }}\right|<0.7$, the estimated detector resolutions in data and MC agree very well within $\pm 8 \%$. Therefore, an uncertainty of $\pm 8 \%$ on the jet energy resolution will be considered to deal with the associated systematic uncertainty (see section 4.6). The bisector method shows that the resolution is underestimated in the MC for jets in the regions $0.7<\left|y^{j e t}\right|<1.1$ and $1.6<\left|y^{\text {jet }}\right|<2.1$, while it is overestimated for jet within $1.1<\left|y^{j e t}\right|<1.6$. Those two cases required different methods to correct the discrepancy between data and simulation. In the following the different cases are explained separately.

\subsubsection{Correction for underestimated resolution in the simulation}

The corrected resolution is obtained by smearing the resolution in the $\mathrm{MC}, \sigma_{M C}$, by a Gaussian, $\sigma_{G}: \sigma_{M C}^{C O R R}=\sigma_{M C} \oplus \sigma_{G}$, in order to get

$$
\sigma_{M C}^{C O R R}=\sigma_{M C} \times F \quad \text { with } F>1
$$

where the corresponding correction factor $\mathrm{F}$ is chosen in such a way that the $\sigma_{D}$ values observed in the MC after the smearing are the same than the ones found in the data. Figure 4.25 shows the $p_{T}^{j e t}$ resolutions in the different rapidity regions as obtained in the $\mathrm{MC}, \sigma_{M C}$, by computing directly the $p_{T}$ differences between the calorimeter jets and their associated hadron level jets.

The correction is applied simply by smearing the $p_{T, C A L}^{\text {jet }}$ values in MC by $\sigma_{G}$, defined as:

$$
\sigma_{G}\left[p_{T, C A L}^{j e t}\right]=\sigma_{M C}\left[p_{T, C A L}^{j e t}\right] \cdot \sqrt{\left(F^{2}-1\right)} .
$$

Therefore, the new $p_{T, C A L}^{\text {jet }}$ smeared values are:

$$
p_{T, C A L, S}^{j e t}=p_{T, C A L}^{j e t}+\Delta p_{T, C A L}^{j e t}
$$

where $\Delta p_{T, C A L}^{j e t}$ stands for the smearing contributions. Figures 4.26 and 4.27 show the data/MC $\sigma_{D}$ ratios for the values of $\mathrm{F}$ that were investigated in each of the 2 rapidity regions under study. The final factors $\mathrm{F}$ that will be applied are 1.06 and 1.10 for jets in $0.7<\left|y^{\text {jet }}\right|<1.1$ and $1.6<$ $\left|y^{j e t}\right|<2.1$ respectively, for which the data/MC ratios are consistent with 1 over the whole $p_{T}$ ranges, and within the $\pm 8 \%$ systematic uncertainty considered .

In the following, the $p_{T, C A L}^{j e t}$ values considered in the $\mathrm{MC}$ will be the ones obtained after the smearing, i.e. the $p_{T, C A L, S}^{j e t}$ values. 


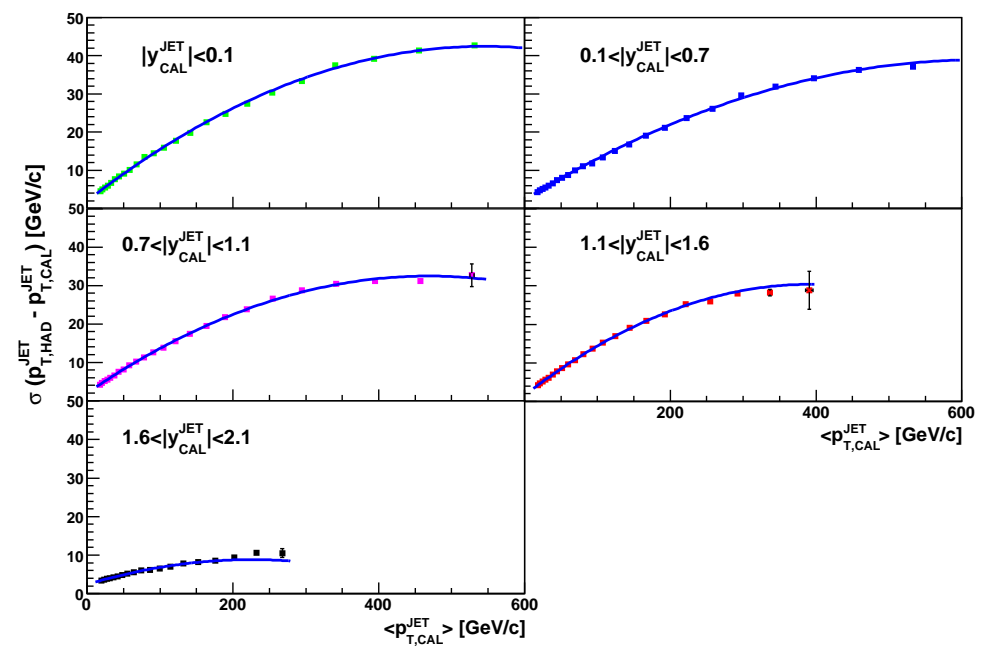

Figure 4.25: $p_{T}^{\text {jet }}$ resolution obtained from the MC comparing hadronic and calorimetric jets for jets in different $\left|y^{j e t}\right|$ regions. The RMS of the $\left(p_{T, H A D}^{j e t}-p_{T, C A L}^{j e t}\right)$ distribution is reported as a function of the $\left\langle p_{T, C A L}^{\text {jet }}>\right.$. The points are fitted by $2^{\text {nd }}$ order polynomials.

\subsubsection{Correction for overestimated resolution in the simulation}

For jets in the rapidity $1.1<\left|y^{\text {jet }}\right|<1.6$, the resolution in the MC is overestimated and the method based on the smearing of the $p_{T, C A L}^{j e t}$ values in the MC cannot be applied. In this case, a resolution correction will be applied later via slightly modified unfolding factors (see section 4.5.2.1).

However, in order to know how different are the resolutions in data and MC, a similar study than the one presented previously has been carried out. In this case, and only to do this study, the smearing was done on the $p_{T, C A L}^{j e t}$ in the data.

Figure 4.28 shows the data/MC $\sigma_{D}$ ratios for the values of $\mathrm{F}$ that were investigated, where $\mathrm{F}$ has been defined as before. Data and $\mathrm{MC}$ agree for $\mathrm{F}=1.05$, therefore the factor that will be applied to correct the resolution in the simulation is $1 / 1.05$. As mentioned earlier, this will be done indirectly via modified unfolding factors. 

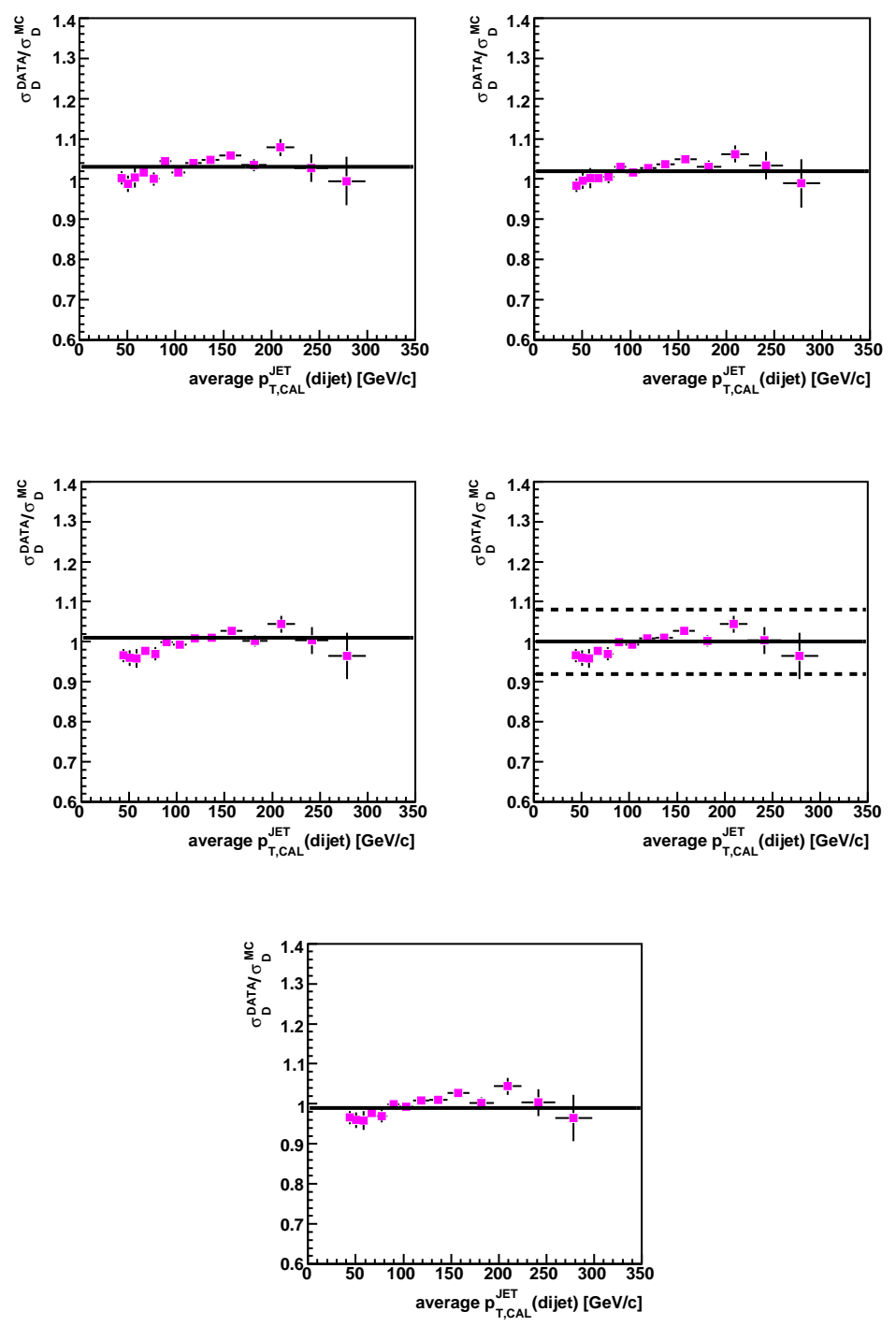

Figure 4.26: Ratio data / MC in $\sigma_{D}$ vs the average $p_{T, C A L}^{j e t}$ of the dijet event as obtained from the bisector method for jets in the region $0.7<\left|y^{\text {jet }}\right|<1.1$ after increasing the resolution by different factors $\mathrm{F}$, from 1.03 to 1.07 by steps of 0.01 . The chosen factor is $\mathrm{F}=1.06$. The solid lines are fits to 0 order polynomials and the dashed lines show the $\pm 8 \%$ uncertainty considered. 

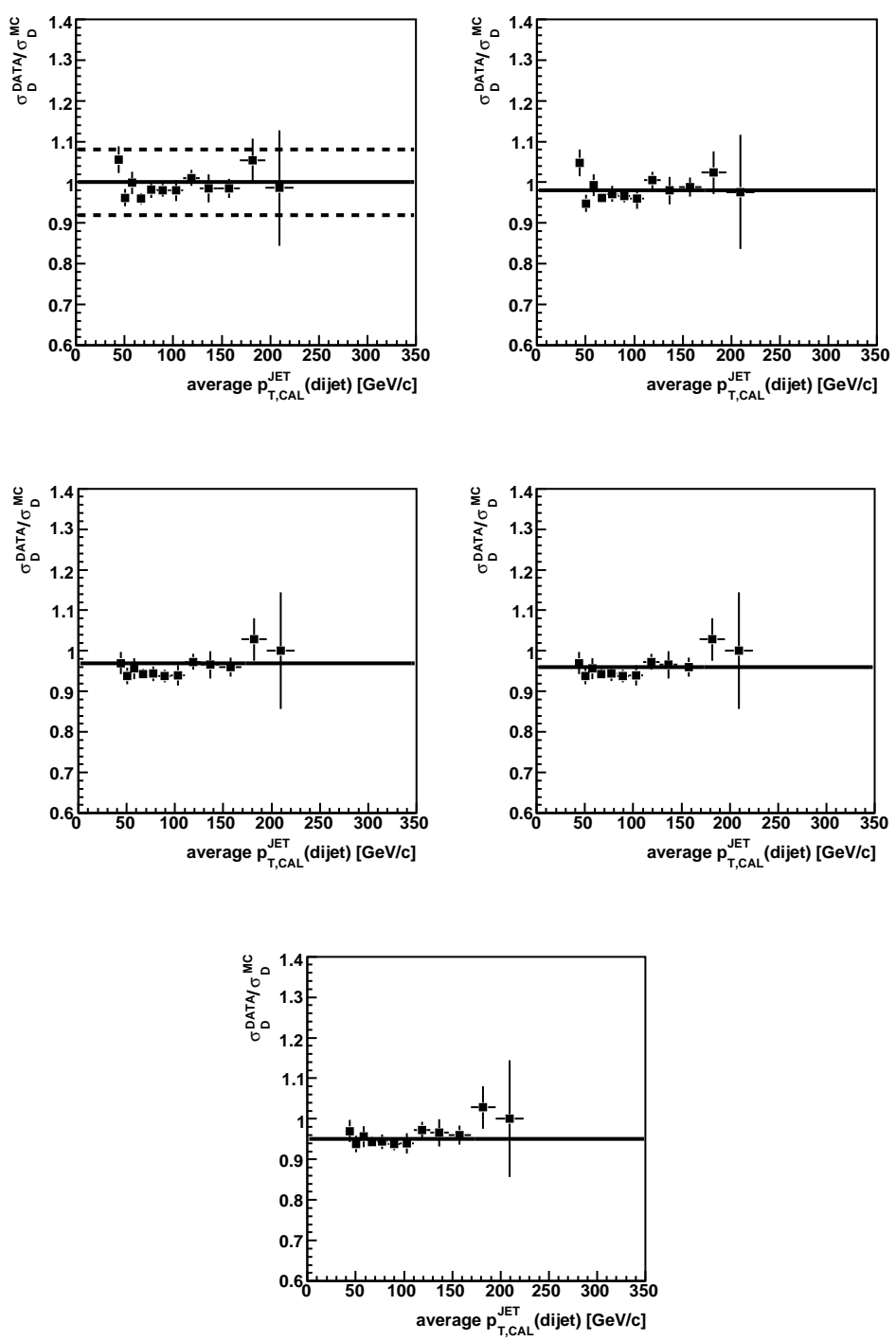

Figure 4.27: Ratio data / MC in $\sigma_{D}$ vs the average $p_{T, C A L}^{j e t}$ of the dijet event as obtained from the bisector method for jets in the region $1.6<\left|y^{\text {jet }}\right|<2.1$ after increasing the resolution by different factors $\mathrm{F}$, from 1.10 to 1.18 by steps of 0.02. The chosen factor is $\mathrm{F}=1.10$. The solid lines are fits to 0 order polynomials and the dashed lines show the $\pm 8 \%$ uncertainty considered. 

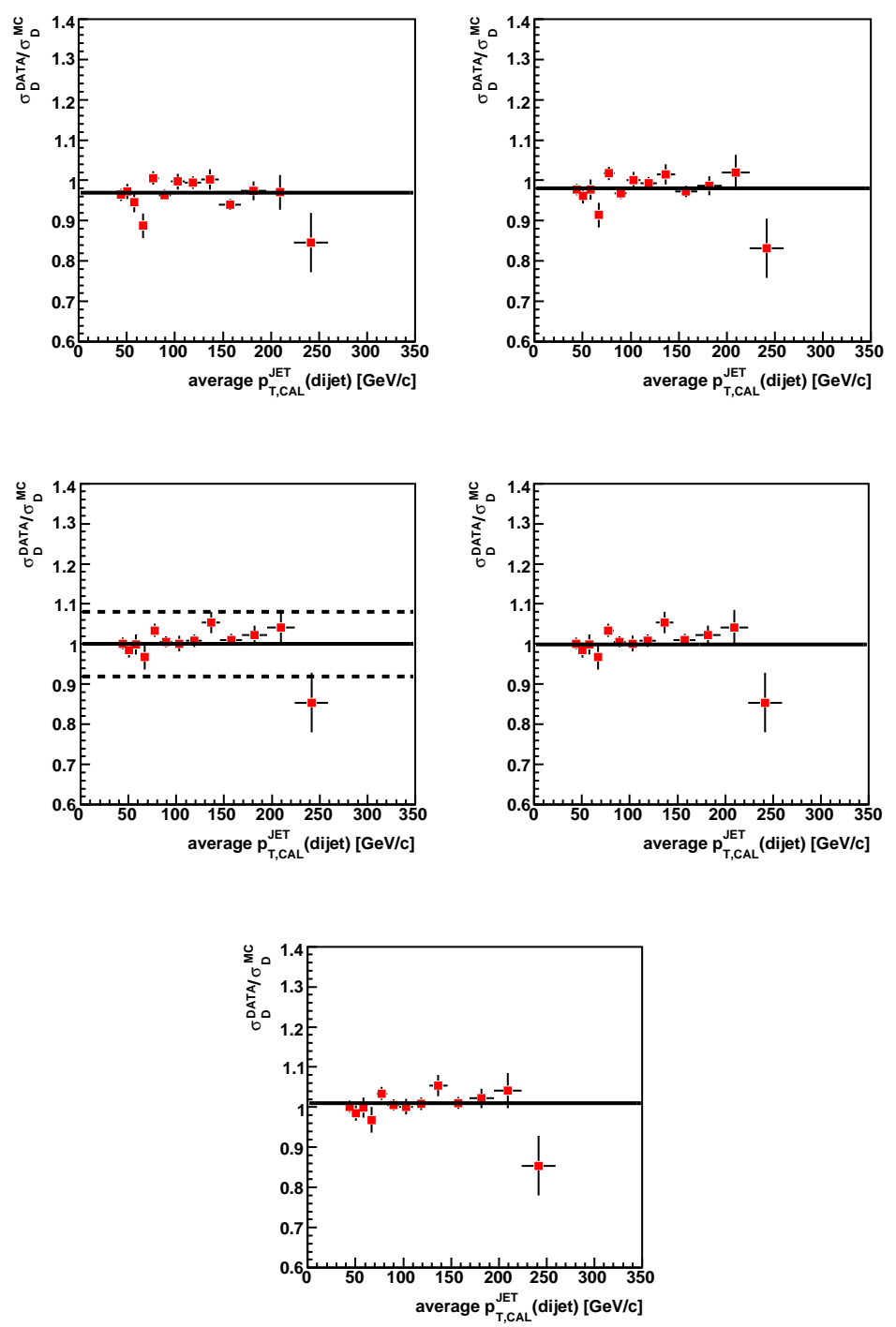

Figure 4.28: Ratio data / $\mathrm{MC}$ in $\sigma_{D}$ vs the average $p_{T, C A L}^{j e t}$ of the dijet event as obtained from the bisector method for jets in the region $1.1<\left|y^{\text {jet }}\right|<1.6$ after smearing the data by different factors $\mathrm{F}$, from 1.03 to 1.07 by steps of 0.01 . The chosen factor is $\mathrm{F}=1.05$. The solid lines are fits to 0 order polynomials and the dashed lines show the $\pm 8 \%$ uncertainty considered. 


\subsubsection{Dijet Balance}

Once the jet momentum resolutions have been adjusted in the $\mathrm{MC}$ according to the ones measured in the data, whenever it was necessary and possible as it was explained in previous section, the description of the average calorimeter response to jets in the MC is tested. A sample of dijet events is selected and the difference between the $p_{T}$ of the two jets is considered to evaluate the jet energy scale relative to central jets.

The event selection is the following:

- 2 and only 2 jets with jet $p_{T, C A L}^{\text {jet }}$ above $10 \mathrm{GeV} / \mathrm{c}$.

- One jet (trigger jet) in the detector $\eta$ region $0.2<\eta_{D}^{J E T}<0.6$, the other jet (probe jet) in the rapidity region under study.

- 1 and only 1 primary vertex with $\left|V_{Z}\right| \leq 60 \mathrm{~cm}$.

- Missing $E_{T}$ significance cut (see section 4.1.2).

The following quantities are defined:

- $p_{T}^{T R I G}=p_{T, C A L}^{\text {jet }}$ (trigger jet) and $p_{T}^{P R O B}=p_{T, C A L}^{\text {jet }}$ (probe jet)

- $p_{T}^{M E A N}=\left(p_{T}^{P R O B}+p_{T}^{T R I G}\right) / 2$

- $\Delta p_{T}^{F}=\left(p_{T}^{P R O B}-p_{T}^{T R I G}\right) / p_{T}^{M E A N}$

The selected events are again separated in bin of $p_{T}^{M E A N}$. For each bin, the mean unbalance fraction $\left\langle\Delta p_{T}^{F}\right\rangle$ is evaluated, and the quantity

$$
\beta=\left(2+<\Delta p_{T}^{F}>\right) /\left(2-<\Delta p_{T}^{F}>\right)
$$

is computed. Event by event $\beta$ is equivalent to $p_{T}^{P R O B} / p_{T}^{T R I G}$. Figure 4.29 shows the data over MC ratio: $\beta_{D A T A} / \beta_{M C}$. For jets in the rapidity regions $0.1<\left|y^{j e t}\right|<0.7$ and $0.7<\left|y^{j e t}\right|<1.1$ the ratio $\beta_{D A T A} / \beta_{M C}$ is compatible with 1 over all the $p_{T}$ range, showing that the MC reproduces very well the data. However, for jets in the central gap and in the two most forward regions, it is different than 1. An additional correction is applied to the $\mathrm{MC}$ in order to force the ratio $\beta_{D A T A} / \beta_{M C}$ to be equal to 1 over the whole $p_{T}$ range. The corrections are obtained fitting the ratio by a polynomial. A constant is used for jets within $\left|y^{j e t}\right|<0.1$, leading to:

$$
p_{T, C A L, c o r}^{j e t}=1.011 \cdot p_{T, C A L}^{j e t} .
$$


For jets within $1.1<\left|y^{j e t}\right|<1.6$ and $1.6<\left|y^{j e t}\right|<2.1$ there are limited statistics for dijet events at high $p_{T, C A L}^{j e t}$. Therefore, different linear functions have been considered to extrapolate the correction to high $p_{T}$ :

$$
\begin{array}{ll}
p_{T, C A L, \text { cor }}^{j e t}=1.006 \cdot p_{T, C A L}^{j e t}-0.00014 \cdot p_{T, C A L}^{2} & \text { for } p_{T, C A L}^{j e t}<110 \mathrm{GeV} / \mathrm{c} \\
p_{T, C A L, \mathrm{cor}}^{j e t}=0.996 \cdot p_{T, C A L}^{j e t}-0.00007 \cdot p_{T, C A L}^{2} & \text { for } p_{T, C A L}^{j e t}>110 \mathrm{GeV} / \mathrm{c}
\end{array}
$$

and

$$
\begin{array}{ll}
p_{T, C A L, c o r}^{j e t}=1.001 \cdot p_{T, C A L}^{j e t}-0.00020 \cdot p_{T, C A L}^{2} & \text { for } p_{T, C A L}^{j e t}<125 \mathrm{GeV} / \mathrm{c} \\
p_{T, C A L, c o r}^{j e t}=0.988 \cdot p_{T, C A L}^{j e t}-0.00010 \cdot p_{T, C A L}^{2} & \text { for } p_{T, C A L}^{j e t}>125 \mathrm{GeV} / \mathrm{c}
\end{array}
$$

for $1.1<\left|y^{j e t}\right|<1.6$ and $1.6<\left|y^{j e t}\right|<2.1$, respectivelly.

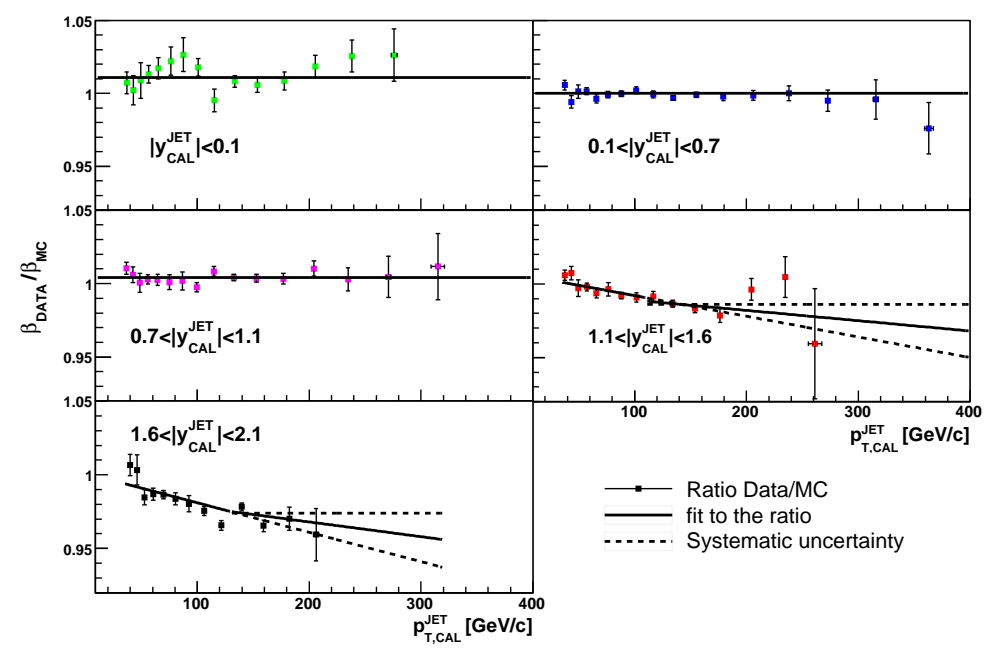

Figure 4.29: Ratio $\beta_{D A T A} / \beta_{M C}$ vs $<p_{T, C A L}^{\text {jet }}>$ for jets in different $\left|y^{\text {jet }}\right|$ regions. The solid lines show the parameterizations that defined the nominal corrections. In the two most forward regions the dashed lines correspond to the different parameterizations used to describe the ratio at high $p_{T, C A L}^{j e t}$. These different functions are considered in the systematic uncertainties studies. 


\subsubsection{Summary of MC corrections}

Table 4.4 summarizes the corrections applied to the MC in the different rapidity regions to obtain a better simulation of the jet energy resolution and average jet energy as determined using the bisector method and the dijet balance studies.

\begin{tabular}{|c|c|c|}
\hline $\begin{array}{c}\text { Rapidity } \\
\text { region }\end{array}$ & $\begin{array}{c}\text { Bisector Method } \\
\sigma_{M C}^{C O R R} / \sigma_{M C}\end{array}$ & $\begin{array}{c}\text { Dijet Balance } \\
p_{T, C A L, c o r}^{\text {jet }}\end{array}$ \\
\hline$\left|y^{\text {jet }}\right|<0.1$ & - & $1.011 \cdot p_{T, C A L}^{\text {jet }}$ \\
\hline $0.1<\left|y^{\text {jet }}\right|<0.7$ & - & - \\
\hline $0.7<\left|y^{\text {jet }}\right|<1.1$ & 1.06 & - \\
\hline $1.1<\left|y^{j e t}\right|<1.6$ & 0.95 & $\begin{array}{c}1.006 \cdot p_{T, C A L}^{j e t}-0.00014 \cdot p_{T, C A L}^{2}\left(p_{T, C A L}^{j e t}<110 \mathrm{GeV} / c\right) \\
\text { or } \\
0.996 \cdot p_{T, C A L}^{j e t}-0.00007 \cdot p_{T, C A L}^{2}\left(p_{T, C A L}^{j e t}>110 \mathrm{GeV} / c\right)\end{array}$ \\
\hline $1.6<\left|y^{j e t}\right|<2.1$ & 1.10 & $\begin{array}{c}1.001 \cdot p_{T, C A L}^{j e t}-0.00020 \cdot p_{T, C A L}^{2}\left(p_{T, C A L}^{j e t}<125 \mathrm{GeV} / \mathrm{c}\right) \\
\text { or } \\
0.988 \cdot p_{T, C A L}^{j e t}-0.00010 \cdot p_{T, C A L}^{2}\left(p_{T, C A L}^{j e t}>125 \mathrm{GeV} / c\right)\end{array}$ \\
\hline
\end{tabular}

Table 4.4: Corrections applied to the MC coming from the bisector method and dijet balance studies. Jet measured in the central region, $0.1<\left|y^{j e t}\right|<0.7$, are perfectly described by the MC. In the same way, data and MC agree on the jet energy resolution for jets within $\left|y^{j e t}\right|<0.1$ and on the jet energy scale relative to central jets in the rapidity range $0.7<\left|y^{j e t}\right|<1.1$.

\subsection{Unfolding to the hadron level}

Once the simulation has been evaluated and eventually corrected, the measured $p_{T, C A L}^{\text {jet }}$ distributions are corrected back to the hadron level using the MC.

Figure 4.30 shows the ratio between the measured $p_{T, C A L}^{j e t}$ distribution and the one predicted by PYTHIA-Tune A. The ratios show a shape in $p_{T}^{j e t}$ which indicates that the $p_{T}$ distribution in the data are not perfectly followed by the MC. The trend at high $p_{T}^{\text {jet }}$ might be explained by the fact that CTEQ5L PDFs are used in PYTHIA-Tune A.

In order to avoid any bias on the correction factors due to the particular PDFs used during the 
generation of the MC samples PYTHIA-Tune A is reweighted to follow the measured jet $p_{T}$ spectrum in the data. The ratio between the measured and predicted distributions is fitted to a third order polynomial. Each event in the MC is then weighted by this third order polynomial applied to $\hat{p}_{T}$.

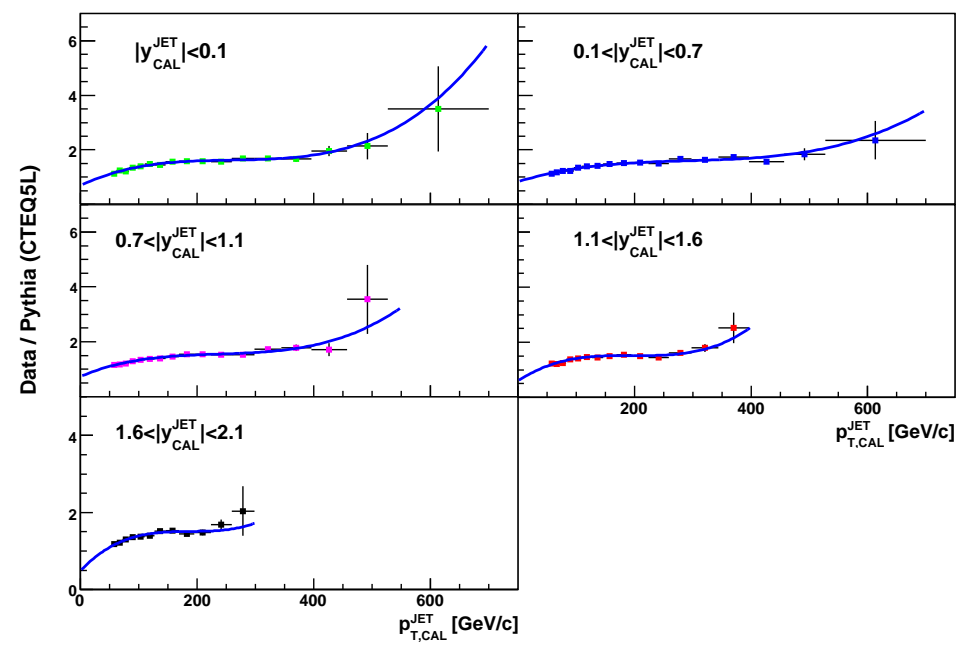

Figure 4.30: Ratio between the measured $p_{T, C A L}^{j e t}$ distribution and the one predicted by PYTHIA-Tune A for jets in different $\left|y^{j e t}\right|$ regions. The ratios are fitted to $3^{\text {rd }}$ order polynomials.

The unfolding is carried out in two steps. First, an average $p_{T}^{\text {jet }}$ correction takes into account in average the energy losses of hadrons going through the non-compensated calorimeters $(e / h \neq 1)$. Second, an unfolding procedure is applied to correct the measurements for acceptance and smearing effects, accounting for the efficiency of the selection criteria and for the jet reconstruction in the calorimeter. Both steps are explained in the next sections. 


\subsubsection{Average $p_{T}^{\text {jet }}$ correction}

The $p_{T}^{\text {jet }}$ average correction is extracted using the following procedure:

- Jets are reconstructed at calorimeter and hadron levels in PYTHIA-Tune A MC.

- Pairs of calorimeter and hadron level jets are matched in $y \times \phi$ space if their separation $\Delta R=\sqrt{\Delta Y^{2}+\Delta \phi^{2}}$ is lower than 0.7. If more than one hadron level jet is within $\Delta R<0.7$, the closest one is retained.

- The correlation $<p_{T, H A D}^{j e t}-p_{T, C A L}^{j e t}>$ versus $\left\langle p_{T, C A L}^{j e t}>\right.$, where $p_{T, H A D}^{j e t}$ is the $p_{T}$ of the hadron level jet and $p_{T, C A L}^{j e t}$ is the $p_{T}$ of the calorimeter jet, is computed for matched pairs of jets. It is then fitted to a fourth order polynomial. The corrected $p_{T}$ of the calorimeter jet is then:

$$
p_{T, C O R}^{j e t}=P_{0}+P_{1} \cdot p_{T, C A L}^{j e t}+P_{2} \cdot p_{T, C A L}^{j e t 2}+P_{3} \cdot p_{T, C A L}^{j e t}+P_{4} \cdot p_{T, C A L}^{j e t} 4
$$

The average quantities $<p_{T, H A D}^{\text {jet }}-p_{T, C A L}^{\text {jet }}>$ and $<p_{T, C A L}^{\text {jet }}>$ are computed in bins of $\left(p_{T, H A D}^{\text {jet }}+\right.$ $\left.p_{T, C A L}^{j e t}\right) / 2$. The obtained corrections are shown in figure 4.31 .

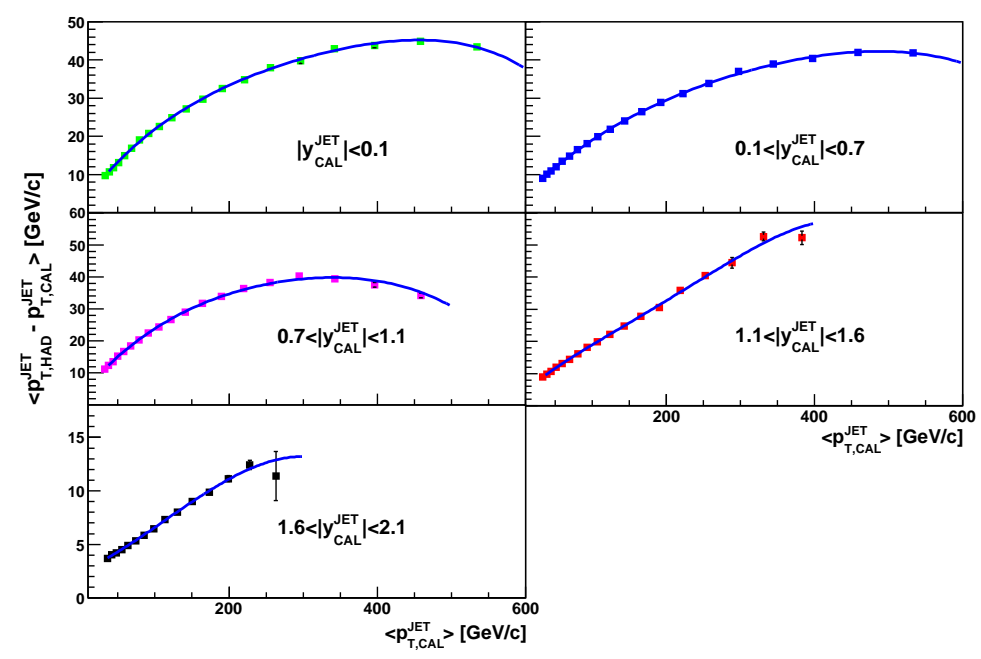

Figure 4.31: Average $p_{T}$ correction, $\left\langle p_{T, H A D}^{\text {jet }}-p_{T, C A L}^{\text {jet }}>\mathrm{vs}\left\langle p_{T, C A L}^{\text {jet }}>\right.\right.$, for jets in different $\left|y^{\text {jet }}\right|$ regions. The correction is fitted to $4^{\text {th }}$ order polynomials (solid lines). 


\subsubsection{Unfolding Procedure}

The unfolding procedure is based again on PYTHIA-Tune A MC:

- The unfolding factors are defined bin-by-bin as:

$$
U\left(p_{T, C O R}^{j e t}, y_{C A L}^{j e t}\right)=\frac{d^{2} / d p_{T, H A D}^{j e t} d y_{H A D}^{j e t}}{d^{2} / d p_{T, C O R}^{j e t} d y_{C A L}^{j e t}} .
$$

- The bin-by-bin unfolding factors are applied to the measured $p_{T}^{\text {jet }}$ distribution to unfold it back to the hadron level:

$N_{J E T}^{D A T A U N F O L D E D}\left(p_{T, C O R}^{\text {jet }}, y_{C A L}^{\text {jet }}\right)=U\left(p_{T, C O R}^{\text {jet }}, y_{C A L}^{\text {jet }}\right) \cdot N_{J E T}^{D A T A N O T}$ UNFOLDED $\left(p_{T, C O R}^{\text {jet }}, y_{C A L}^{\text {jet }}\right)$.

The unfolding factors are obtained by combining different samples of MC with different cuts on $\hat{p}_{T}$. As in all the MC studies, special care has been taken at this point to avoid any bias due to the presence of the $\hat{p}_{T}$ thresholds. Selected MC samples for different $p_{T}^{\text {jet }}$ bins and rapidity regions are reported in table 4.5. Figure 4.32 shows the unfolding factors.

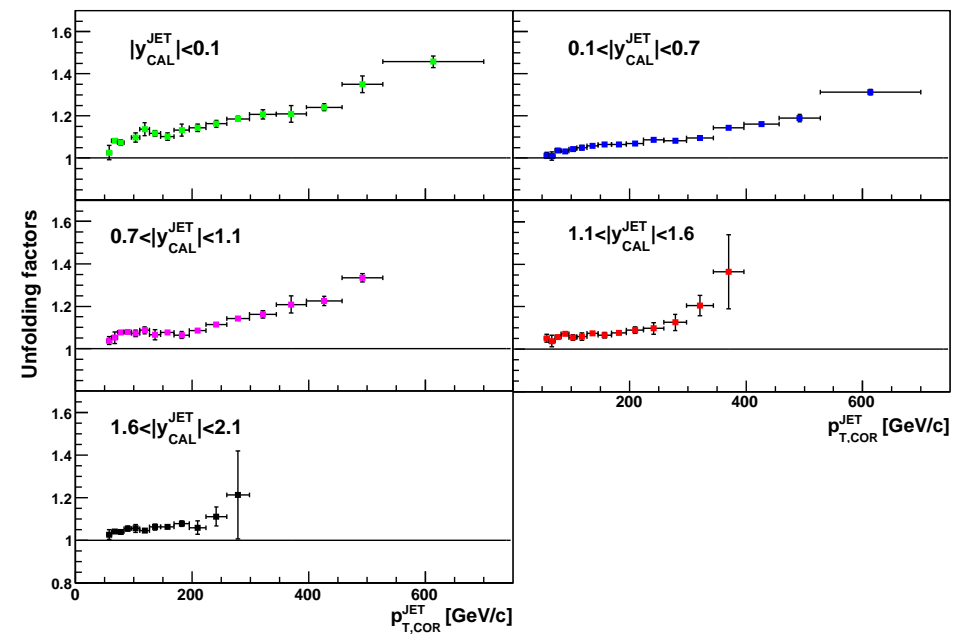

Figure 4.32: Bin-by-bin unfolding factors vs $p_{T, C O R}^{\text {jet }}$ obtained using reweighted PYTHIA-Tune A for jets in different $\left|y^{\text {jet }}\right|$ regions. 


\begin{tabular}{|c|c|c|c|c|c|}
\hline$p_{T, C O R}^{\text {jet }}$ & $\left|y^{\text {jet }}\right|<0.1$ & $0.1<\left|y^{\text {jet }}\right|<0.7$ & $0.7<\left|y^{\text {jet }}\right|<1.1$ & $1.1<\left|y^{\text {jet }}\right|<1.6$ & $1.6<\left|y^{\text {jet }}\right|<2.1$ \\
\hline $54-62$ & PYT18 & PYT18 & PYT18 & PYT18 & PYT18 \\
\hline $62-72$ & PYT40 & PYT18 & PYT18 & PYT18 & PYT40 \\
\hline $72-83$ & PYT40 & PYT40 & PYT18 & PYT40 & PYT40 \\
\hline $83-96$ & PYT40 & PYT40 & PYT40 & PYT60 & PTY60 \\
\hline $96-110$ & PYT60 & PYT60 & PYT40 & PYT60 & PYT60 \\
\hline $110-127$ & PYT60 & PYT60 & PYT40 & PYT60 & PYT90 \\
\hline $127-146$ & PYT90 & PYT90 & PYT60 & PYT90 & PYT90 \\
\hline $146-169$ & PYT90 & PYT90 & PYT90 & PYT90 & PYT120 \\
\hline $169-195$ & PYT90 & PYT120 & PYT90 & PYT120 & PYT150 \\
\hline $195-224$ & PYT120 & PYT150 & PYT120 & PYT120 & PYT150 \\
\hline $224-259$ & PYT150 & PYT150 & PYT150 & PYT120 & PYT200 \\
\hline $259-298$ & PYT200 & PYT150 & PYT150 & PYT150 & PYT200 \\
\hline $298-344$ & PYT200 & PYT200 & PYT200 & PYT200 & - \\
\hline $344-396$ & PYT200 & PYT200 & PYT200 & PYT200 & - \\
\hline $396-457$ & PYT300 & PYT300 & PYT300 & - & - \\
\hline $457-527$ & PYT300 & PYT300 & PYT400 & - & - \\
\hline $527-700$ & PYT400 & PYT400 & PYT400 & - & - \\
\hline
\end{tabular}

Table 4.5: PYTHIA-Tune A MC samples used in each $p_{T, C O R}^{\text {jet }}$ bin to evaluate the unfolding corrections in the different rapidity regions.

\subsubsection{Resolution correction}

As it has been shown in section 4.4.2, the resolution for jets in the rapidity $1.1<\left|y^{\text {jet }}\right|<1.6$ is overestimated in the MC. In this case, a smearing of the $p_{T, C A L}^{j e t}$ values cannot be applied. An alternative method has been used, where the unfolding factors are corrected in order to indirectly take into account the discrepancy between data and $\mathrm{MC}$ on the jet momentum resolution. The correction factors are extracted from the ratio of the $p_{T, H A D}^{j e t}$ spectrum smeared by the nominal $\mathrm{MC}$ resolution, $\sigma_{M C}$, and by the corrected resolution defined as $\sigma_{M C}^{C O R R}=\sigma_{M C} \times(1 / 1.05)$ (see section 4.4.2.2). The correction to be applied to the unfolding factors is about $3 \%$ and essentially independent of $p_{T}^{\text {jet }}$. Figure 4.33 shows the corrections together with the resulting final unfolding factors. 

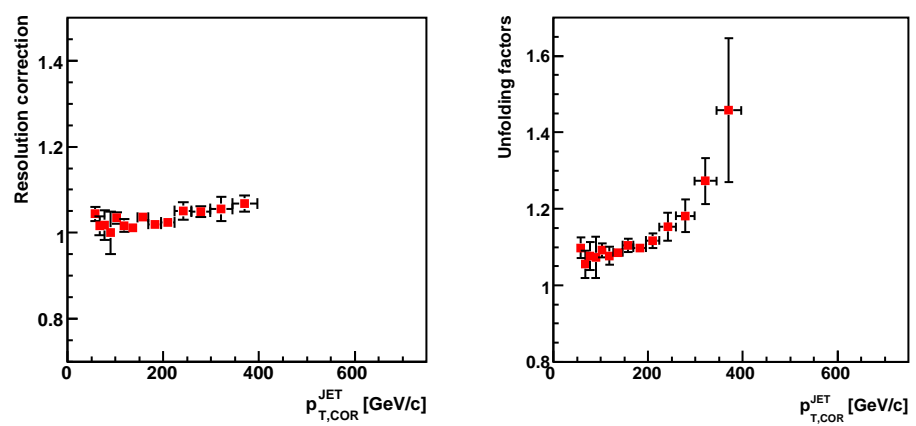

Figure 4.33: Resolution correction factors (left) and modified unfolding factors (right) vs $p_{T, C O R}^{j e t}$ for jets in the region $1.1<\left|y^{\text {jet }}\right|<1.6$.

The method explained here to account for the discrepancies between data and MC on the jet momentum resolution has been validated in the regions $0.7<\left|y^{\text {jet }}\right|<1.1$ and $1.6<\left|y^{\text {jet }}\right|<2.1$ where the resolutions in the MC are underestimated and where the nominal method is to smear the MC $p_{T, C A L}^{j e t}$ distribution directly. The two different correction methods produce compatible results.

It should be noted that for the region $1.1<\left|y^{j e t}\right|<1.6$ the dijet balance study is performed before correcting the MC for the resolution. On the other hand, in the regions $0.7<\left|y^{j e t}\right|<1.1$ and $1.6<\left|y^{j e t}\right|<2.1$, the dijet balance studies are done after including the $p_{T, C A L}^{j e t}$ smearing. This is the correct way to proceed as resolution discrepancies between data and $\mathrm{MC}$ can slightly affect the ratio $\beta_{D A T A} / \beta_{M C}$. A detailed study was carried out in the region $1.6<\left|y^{j e t}\right|<2.1$ to evaluate the effect of applying the dijet balance correction before and after the resolution correction. The effect is about $3 \%$ and will be included as an additional systematic uncertainty on the final measurement for the region $1.1<\left|y^{j e t}\right|<1.6$. This is rather conservative since the observed difference in jet momentum resolution between data and $\mathrm{MC}$ in the region $1.6<\left|y^{j e t}\right|<2.1$ is much bigger than for jets within $1.1<\left|y^{j e t}\right|<1.6$. 


\subsection{Systematic Uncertainties}

The following sources of systematic uncertainties have been considered for each cross section measurements:

\section{Jet Energy Scale}

The jet energy scale uncertainty is estimated to be between $\pm 2 \%$ at low $p_{T}^{j e t}$ to $\pm 2.7 \%$ at high $p_{T}^{\text {jet }}$. It mainly comes from the remaining uncertainties on the calorimeter response to single particles and on the $p_{T}$ spectrum of the particles inside the jets [58]. To estimate the associated systematic uncertainties in the cross section, the energy scale is varied in the reweighted MC. This introduces uncertainties on the final measurements between $\pm 10 \%$ at low $p_{T}^{\text {jet }}$ and $40 \%$ to $60 \%$ at high $p_{T}^{\text {jet }}$, depending on the rapidity region, as it is shown in figure 4.34 , which dominate the total systematic uncertainties.

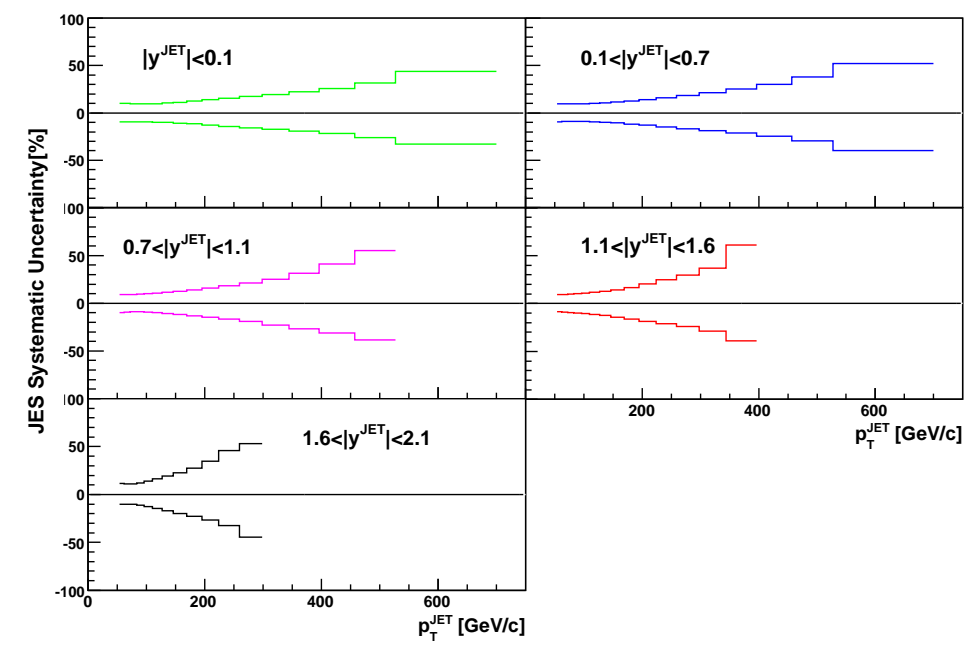

Figure 4.34: Systematic uncertainty (in percentage) on the measured inclusive jet cross sections as a function of $p_{T}^{j e t}$ associated to the uncertainty on the Jet Energy Scale for the different $\left|y^{\text {jet }}\right|$ regions. 


\section{Dijet balance method and correction}

Several source of systematic uncertainties on the dijet balance method have been studied:

- (a) The dijet balance method is based on exclusive dijet events. The veto required for the $3^{\text {rd }}$ jet introduces a $\pm 0.5 \%$ uncertainty on the jet energy scale in all the rapidity regions, excepts for the $0.1<\left|y^{j e t}\right|<0.7$. This translates into an uncertainty on the cross section measurements between $\pm 2 \%$ at low $p_{T}^{\text {jet }}$ and $\pm 10 \%$ at high $p_{T}^{\text {jet }}$, see figure 4.35 .

- (b) For jets in the region $1.1<\left|y^{\text {jet }}\right|<1.6$ and $1.6<\left|y^{\text {jet }}\right|<2.1$ different parametrizations for the $\beta_{D A T A} / \beta_{M C}$ ratios have been considered (see figure 4.29). The associated systematic uncertainties in the measurements are between $5 \%$ at low $p_{T}^{j e t}$ and $20 \%$ at high $p_{T}^{j e t}$ as it is shown in figure 4.36 .

- (c) As mentioned in section 4.5.2.1, in the region $1.1<\left|y^{j e t}\right|<1.6$ a systematic uncertainty of $-3 \%$ is included to take into account variation in the dijet balance results due to the uncorrected overestimated jet momentum resolution.

\section{Jet Energy Resolution}

A $\pm 8 \%$ uncertainty on the jet momentum resolution, as determined from the bisector method study (see section 4.4.2), has been considered. In order to determine its effect in the measurement, the $p_{T, H A D}^{j e t}$ spectrum in the MC is smeared by it nominal resolution $\sigma_{M C}$ and by a modified resolution varied by $\pm 8 \%\left(\sigma\left[p_{T, H A D}^{\text {jet }}\right] \times 0.92\right.$ and $\left.\sigma\left[p_{T, H A D}^{\text {jet }}\right] \times 1.08\right)$. To unfold the corresponding smeared spectra $\left(N_{J E T}^{S 1.00}, N_{J E T}^{S 0.92}\right.$ and $N_{J E T}^{S 1.08}$ respectively) back to the hadron level $\left(N_{J E T}^{H A D}\right)$, one would consider the ratios:

$$
C_{S 1.00}=N_{J E T}^{H A D} / N_{J E T}^{S 1.00}, C_{S 0.92}=N_{J E T}^{H A D} / N_{J E T}^{S 0.92} \text { and } C_{S 1.08}=N_{J E T}^{H A D} / N_{J E T}^{S 1.08}
$$

The systematic uncertainty coming from the resolution uncertainty is then related to the unfolding factor ratios $C_{S 1.08} / C_{S 1.00}$ and $C_{S 0.92} / C_{S 1.00}$. In these ratios, $N_{J E T}^{H A D}$ cancels out and one remains with $N_{J E T}^{S 1.00} / N_{J E T}^{S 1.08}$ and $N_{J E T}^{S 1.00} / N_{J E T}^{S 0.92}$. The relative differences between the smeared spectra then give the systematic uncertainties which are about $\pm 2 \%$ to $\pm 12 \%$, as figure 4.37 shows. 


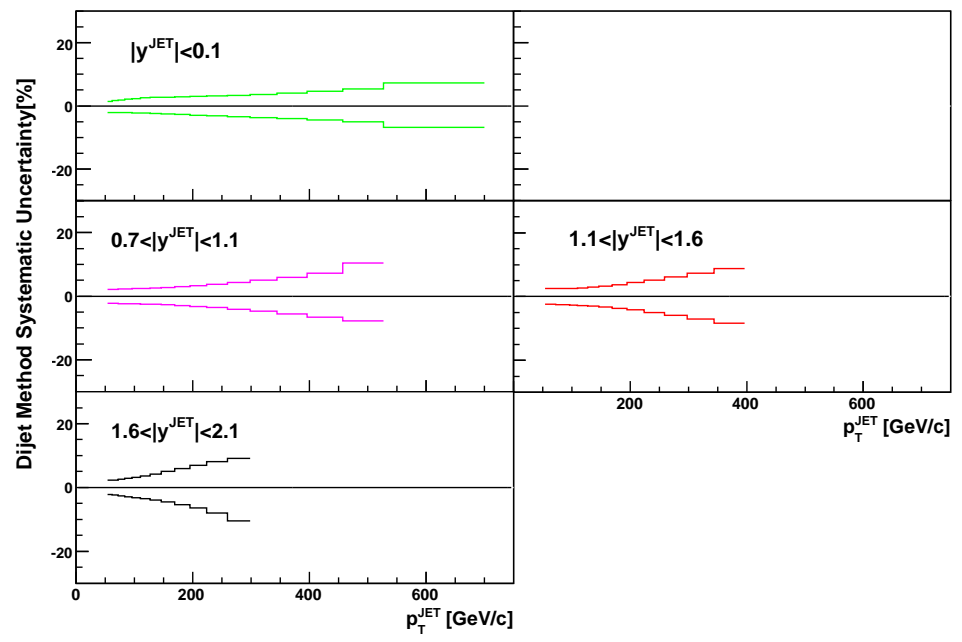

Figure 4.35: Systematic uncertainty (in percentage) on the measured inclusive jet cross sections as a function of $p_{T}^{\text {jet }}$ associated to the uncertainty on the Dijet Method procedure for the different $\left|y^{\text {jet }}\right|$ regions, except $0.1<\left|y^{j e t}\right|<0.7$.

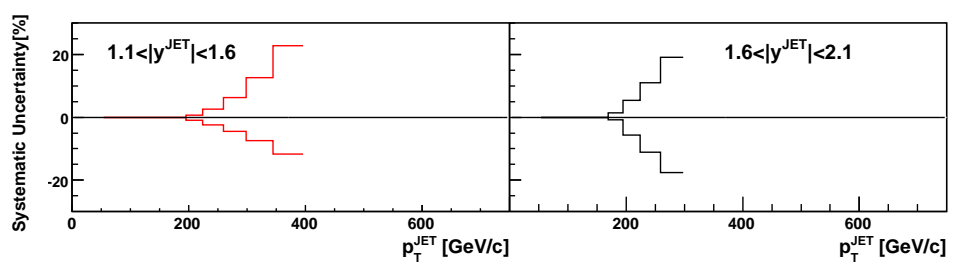

Figure 4.36: Systematic uncertainty (in percentage) on the measured inclusive jet cross sections as a function of $p_{T}^{j e t}$ associated to the uncertainty on the Dijet Method correction for the regions $1.1<\left|y^{\text {jet }}\right|<1.6$ and $1.6<\left|y^{\text {jet }}\right|<2.1$. 


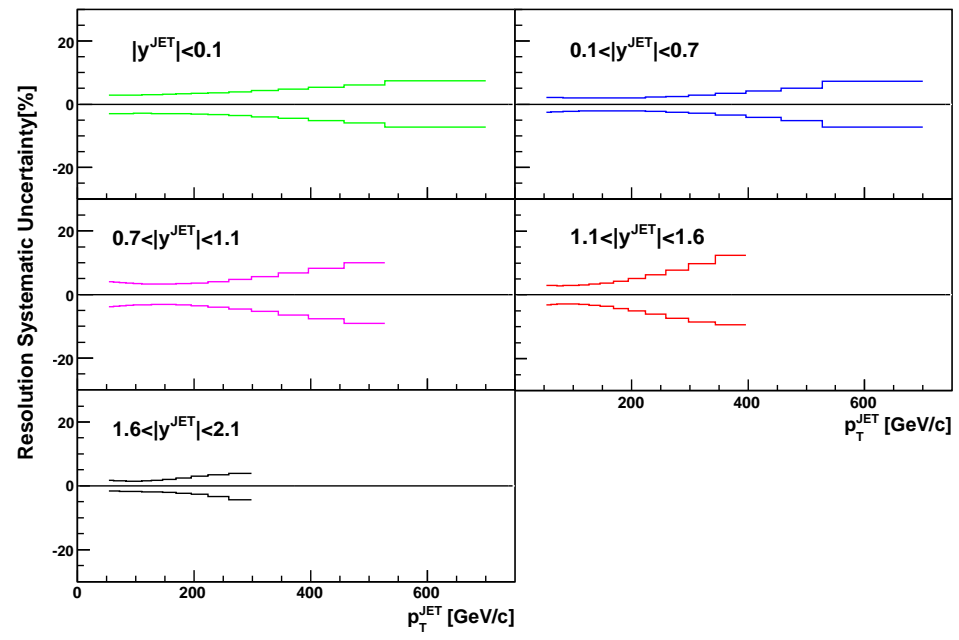

Figure 4.37: Systematic uncertainty (in percentage) on the measured inclusive jet cross sections as a function of $p_{T}^{j e t}$ associated to the uncertainty on the resolution for the different $\left|y^{j e t}\right|$ regions.

\section{Unfolding procedure}

Two different sources of systematic uncertainties are considered:

- A possible remaining sensitivity of the unfolding factors to the jet $p_{T}$ spectrum is estimated by the ratio of the unfolding factors as obtained from unweighted and weighted PYTHIATune A. As it is shown in figures 4.38 , the effect is negligible up to about $400 \mathrm{GeV} / \mathrm{c}$. For higher $p_{T}^{\text {jet }}$ the systematic uncertainty is about $\pm 4 \%$ to $\pm 7 \%$.

- The sensitivity to the hadronization model is accounted by the ratio of the unfolding factors from weighted PYTHIA-Tune A and HERWIG, where for the latter the same weighted method than for PYTHIA-Tune A has been used. Figure 4.39 shows that the biggest deviations are observed at lower $p_{T}^{\text {jet }}$, where they are of the order of $\pm 2 \%$ to $\pm 8 \%$. 


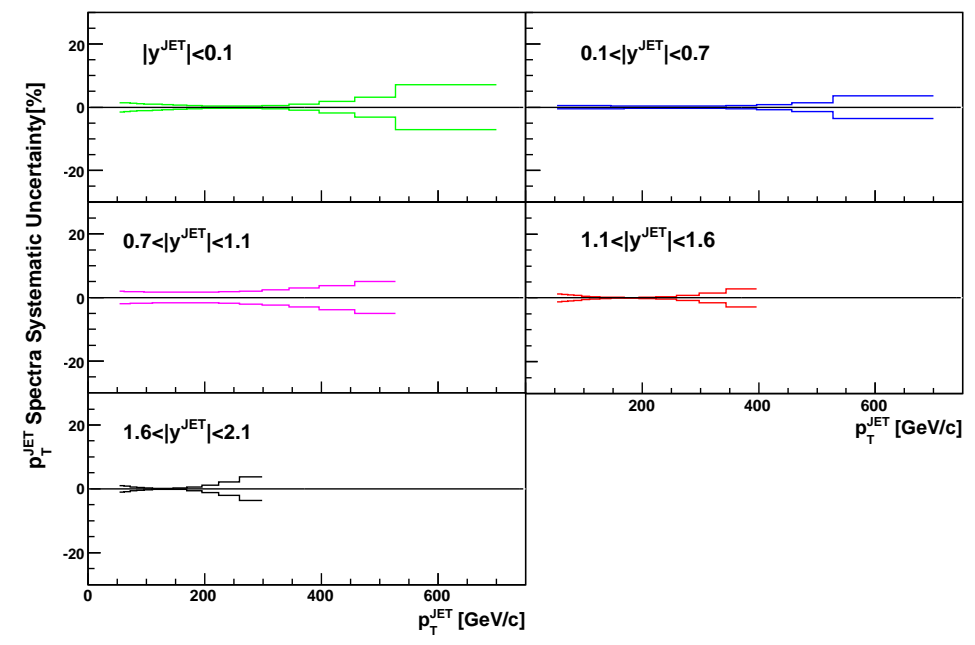

Figure 4.38: Systematic uncertainty (in percentage) on the measured inclusive jet cross sections as a function of $p_{T}^{j e t}$ associated to the uncertainty on the $p_{T}$ spectra for the different $\left|y^{j e t}\right|$ regions.

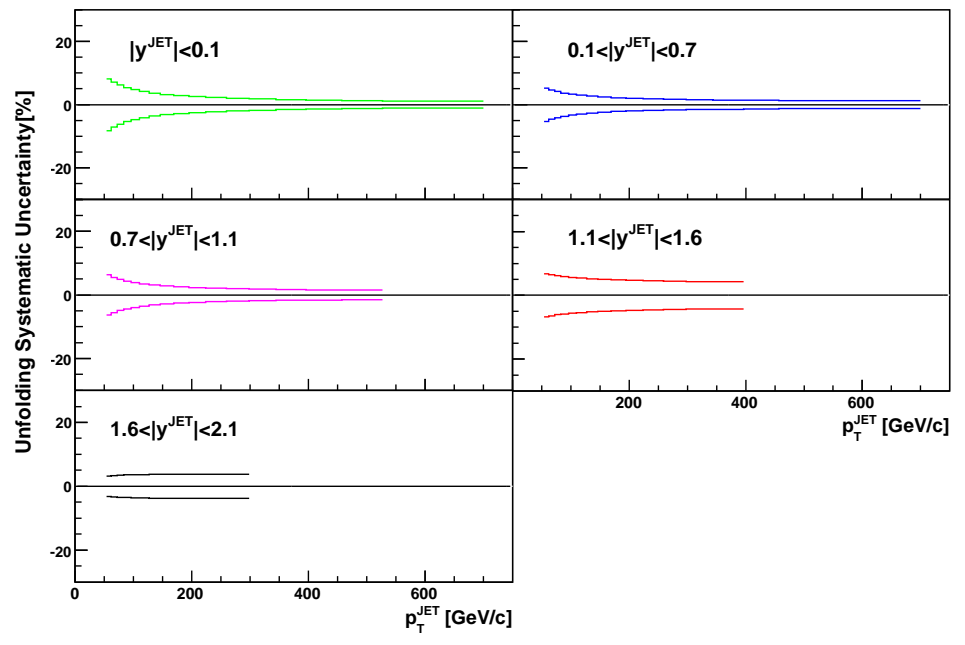

Figure 4.39: Systematic uncertainty (in percentage) on the measured inclusive jet cross sections as a function of $p_{T}^{j e t}$ associated to the uncertainty on the unfolding procedure for the different $\left|y^{\text {jet }}\right|$ regions. 


\section{Pile-up correction}

The pile-up corrections are changed within the systematic uncertainties obtained in the dedicated study (see section 4.3). The effect in the cross section is about $2 \%$ as shown in figure 4.40 .

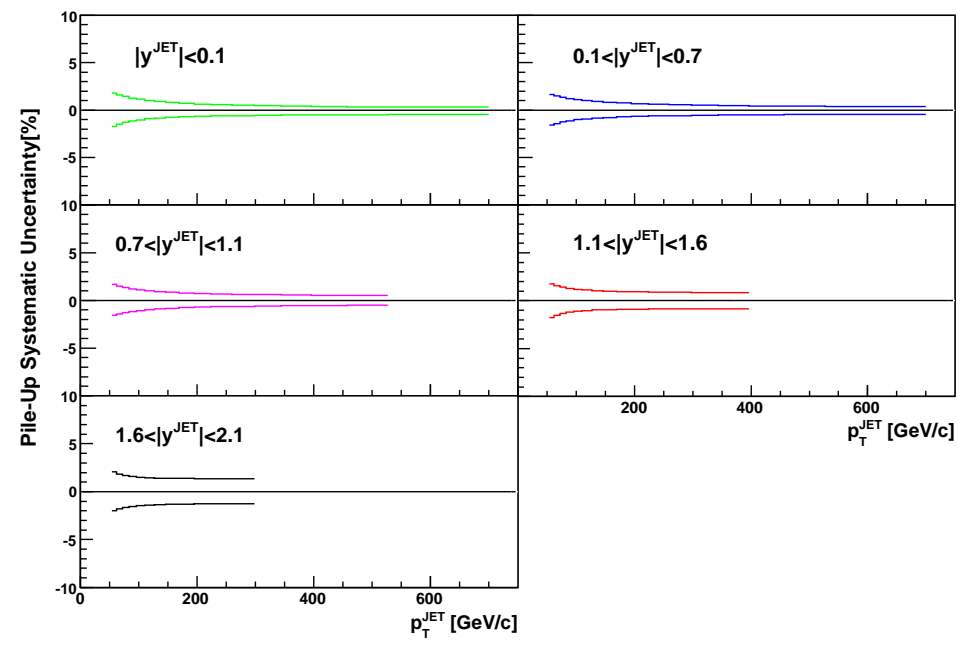

Figure 4.40: Systematic uncertainty (in percentage) on the measured inclusive jet cross sections as a function of $p_{T}^{\text {jet }}$ associated to the uncertainty on the pile-up correction for the different $\left|y^{j e t}\right|$ regions.

\section{Selection criteria}

Three different sources of systematic uncertainties related to the event selection criteria have been considered.

- $V_{Z}$ cut: the $\left|V_{Z}\right|$ cut is varied by $\pm 5 \mathrm{~cm}$ in data and $\mathrm{MC}$ to account for possible remaining differences in the tails of the vertex distribution. The associated systematic uncertainty is $0.3 \%$.

- $p_{T}^{\text {jet }}$ cut: the lowest edge of each bin of the measured jet transverse momentum is varied by $\pm 3 \%$ in both data and MC to identify a possible dependency due to any remaining differences on the $p_{T}^{j e t}$ spectrum in MC and data. The yields change by $30 \%$ to $60 \%$, but data and MC agree very well and, after unfolding, the remaining effect is only about $1 \%$. 
- Missing $E_{T}$ Scale: the energy scale of the missing $E_{T}$ is varied by $\pm 10 \%$ in the data at the same time that the jet energy scale is varied by $\pm 3 \%$ because the missing $E_{T}$ cut is function of $p_{T}^{j e t}$. All possible combinations have been considered and the ones with the biggest effects in each direction are used as a systematic uncertainty. This introduces an uncertainty smaller than $1 \%$.

The contribution of these systematic uncertainties are smaller than $1 \%$, therefore they are considered negligible.

Positive and negative deviations with respect to the nominal value are separated and then added in quadrature. Figure 4.41 shows the total systematic uncertainty, totally dominated by the jet energy scale uncertainty. Tables C.1 to C.5 (see appendix C) summarize the systematic uncertainties coming from each contribution. An additional $\pm 5.8 \%$ normalization uncertainty coming from the luminosity is not included.

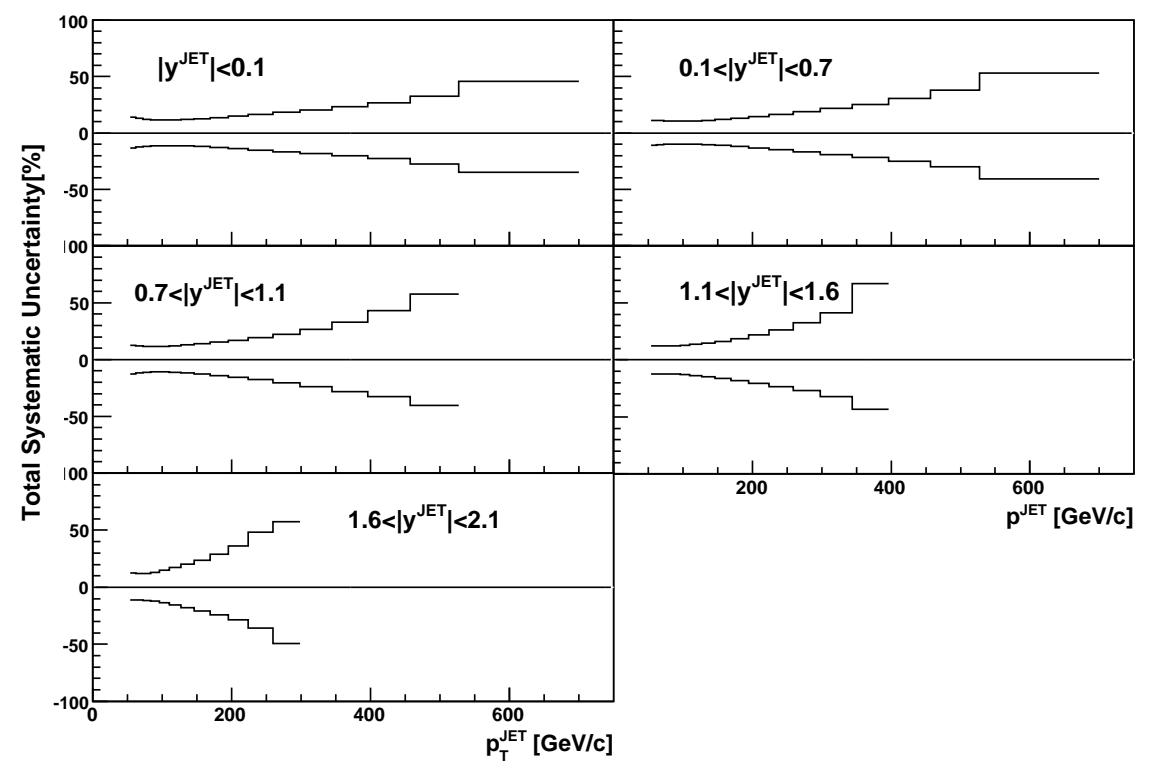

Figure 4.41: Total systematic uncertainty (in percentage) on the measured inclusive jet cross sections as a function of $p_{T}^{\text {jet }}$ for the different $\left|y^{j e t}\right|$ regions. 


\section{Chapter 5}

\section{Discussion of the Results}

The comparison of the jet measurements with the NLO predictions is presented in this chapter. The first section describes the theoretical calculations and their uncertanties. Next, the results are shown and discussed.

\subsection{NLO calculations}

The NLO calculations are obtained from JETRAD program [60] with CTEQ6.1M PDFs. The renormalization and factorization scales, $\mu_{R}$ and $\mu_{F}$ respectively, are set to $\mu_{0}=\max \left(p_{T}^{j e t} / 2\right)$. The effect of changing the scales has been studied and the difference on the theoretical predictions is only few percent.

The uncertainties on the theoretical predictions are dominated by the uncertainties on the PDFs, specially on the gluon contribution at high-x. To compute the PDFs uncertainties, the + and - deviations along the 20 eigenvectors of CTEQ6.1M PDFs are considered. Figure 5.1 shows the 20 eigenvectors for jets in $0.1<\left|y^{\text {jet }}\right|<0.7$. For each one, the two curves are the positive and negative displacements. Asymmetric uncertainties are obtained by summing in quadrature the maximal deviations in each direction associated to each of the 20 eigenvectors. For a given $p_{T}^{j e t}$ bin, calling $P_{0}$ the nominal prediction and $P_{i}^{+}\left(P_{i}^{-}\right)$the prediction corresponding to the $+(-)$ deviation along eigenvector $i$ ( $i$ going from 1 to 20), the global PDFs uncertainties are:

$$
\Delta P_{+}=\left(\sum_{i=1}^{20}\left[\max \left(P_{i}^{+}, P_{i}^{-}, P_{0}\right)-P_{0}\right]^{2}\right)^{1 / 2}
$$


and

$$
\Delta P_{-}=\left(\sum_{i=1}^{20}\left[\min \left(P_{i}^{+}, P_{i}^{-}, P_{0}\right)-P_{0}\right]^{2}\right)^{1 / 2}
$$

If $P_{i}^{+}$and $P_{i}^{-}$give deviations of opposite directions with respect to $P_{0}$, one will contribute to $\Delta P_{+}$ and the other to $\Delta P_{-}$. If $P_{i}^{+}$and $P_{i}^{-}$give deviations in the same direction with respect to $P_{0}$, only the maximal deviation is considered in the corresponding direction: this eigenvector $i$ will not contribute to the opposite direction uncertainty. Figure 5.2 illustrates the global PDFs uncertainty in the theoretical prediction.
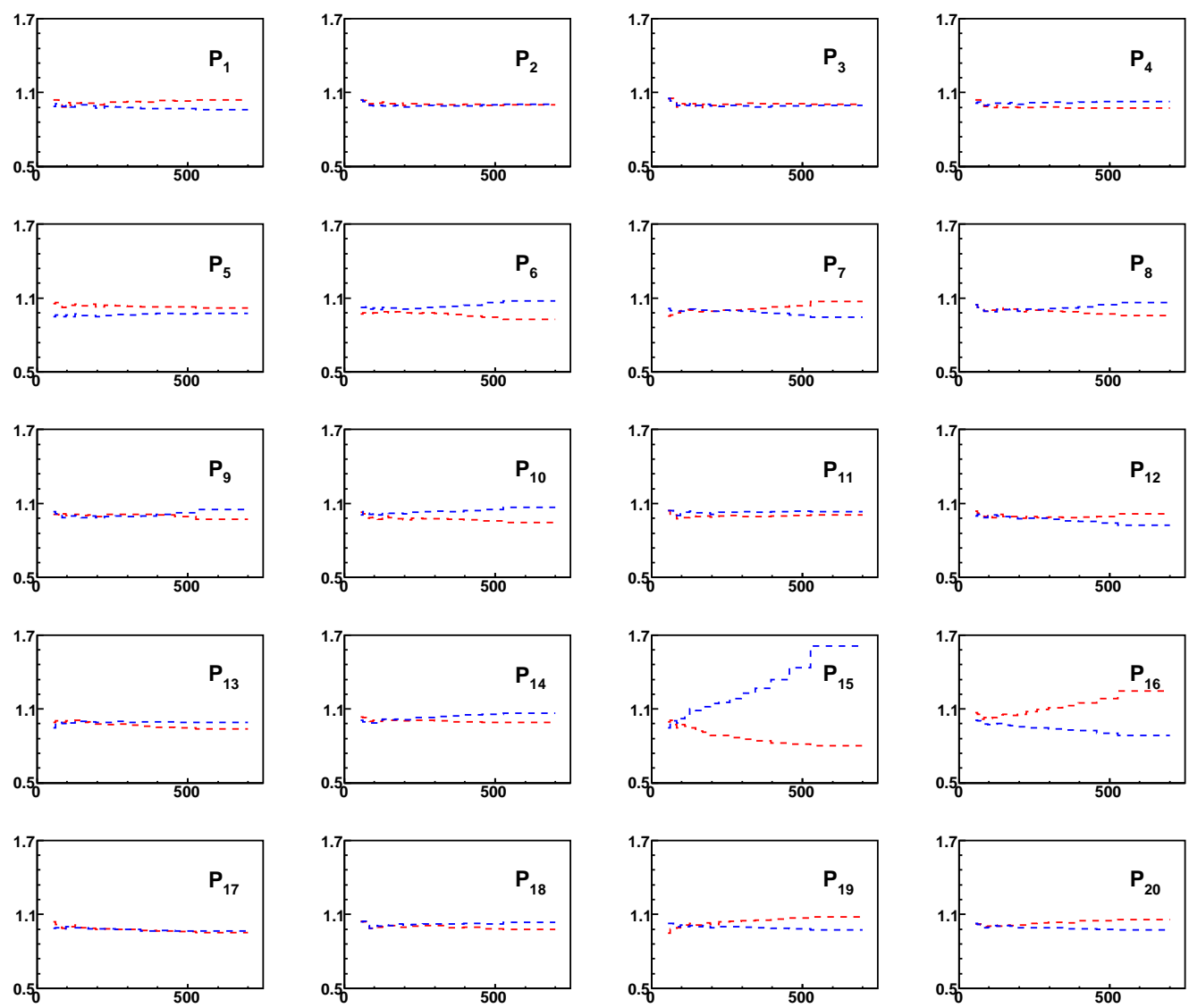

Figure 5.1: Fractional difference between the + and - displacements along the 20 eigenvectors and the standard prediction, CTEQ6.1M, for jet in $0.1<\left|y^{j e t}\right|<0.7$ region. The biggest uncertainty at high $p_{T}$ is related to the gluon contribution, which is the eigenvector 15 in the framework of CTEQ6.1M PDFs. 


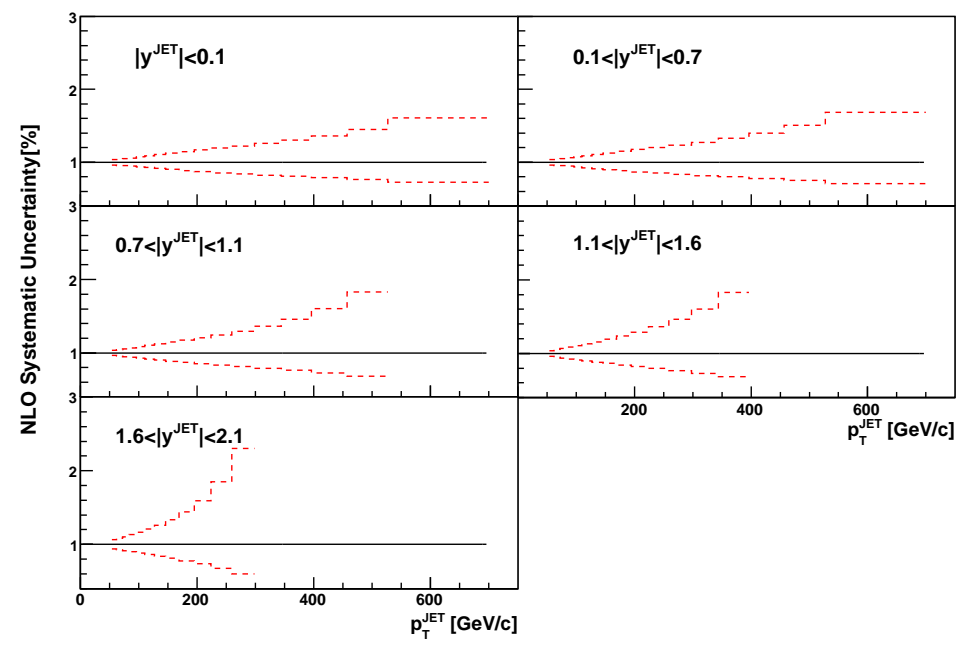

Figure 5.2: Systematic uncertainty (in percentage) on the inclusive jet cross sections predictions, as a function of $p_{T}^{j e t}$, associated to the uncertainty on the PDFs for the different $\left|y^{j e t}\right|$ regions.

\subsubsection{Hadronization and Underlying event correction}

pQCD NLO calculations for the inclusive jet production only have 2 or 3 partons in the final states. This parton-level calculations do not take into account the non-perturbative effects related to the underlying event and the fragmentation processes. Therefore, a correction is necessary for an adequate comparison between the measured jet cross sections at the hadron level and the QCD predictions.

PYTHIA-Tune A is used to estimate this correction. These global underlying event / hadronization correction factors, $C_{H A D}$, are obtained as the difference between the nominal $p_{T}^{\text {jet }}$ cross sections at hadron level and the ones obtained after turning off the multiple parton interaction, which includes beam remnants, and the fragmentation into hadrons in the MC:

$$
\mathrm{C}_{\mathrm{HAD}}\left(\mathrm{p}_{\mathrm{T}}^{\mathrm{jet}}, \mathrm{y}^{\mathrm{jet}}\right)=\frac{\sigma(\text { Hadron level with MPI })}{\sigma(\text { Parton level no MPI })}\left(\mathrm{p}_{\mathrm{T}}^{\mathrm{jet}}, \mathrm{y}^{\mathrm{jet}}\right)
$$


To estimate the different contributions, $C_{H A D}$ can be factorized as following:

$$
\mathrm{C}_{\mathrm{HAD}}=\mathrm{C}_{\mathrm{MPI}}^{\text {Hadron Level }} \times \mathrm{C}_{\text {Frag }}^{\mathrm{No} \text { MPI }}
$$

where:

- $\mathrm{C}_{\mathrm{MPI}}^{\text {Hadron Level }}=[\sigma($ Hadron level with MPI $)] /[\sigma($ Hadron level no MPI $)]$ is the MPI correction obtained at hadron level.

- $\mathrm{C}_{\mathrm{Frag}}^{\text {No MPI }}=[\sigma($ Hadron level no MPI $)] /[\sigma($ Parton level no MPI $)]$ is the fragmentation correction obtained without MPI.

To obtain those corrections, samples with more than 160 millions of events of PYTHIA-Tune A MC with and without MPI have been used. Different $\hat{p}_{T}$ MC samples are used in different $p_{T}^{\text {jet }}$ bins checking carefully that there is no bias due to the $\hat{p}_{T}$ cut by requiring coherence with lowest $\hat{p}_{T}$ samples. Figure 5.3 shows the obtained correction factors, where the fitted values are the one used in the analysis, and its factorization into the two contributions as a function of $p_{T}^{\text {jet }}$ for jets with $0.1<\left|y^{j e t}\right|<0.7$. The correction decreases as $p_{T}^{\text {jet }}$ increases and at low $p_{T}^{\text {jet }}$ the value is approximately 1.2. The corrections have been determined down to $54 \mathrm{GeV} / \mathrm{c}$. At lower $p_{T}^{\text {jet }}$ the correspondence between parton-to-hadron is not well defined and these non-perturbative corrections can not be obtained. For this reason, the measurements and their comparison to NLO are limited to $p_{T}^{\text {jet }} \geq 54 \mathrm{GeV} / \mathrm{c}$.

To account for the systematic uncertainty coming from the modeling of the underlying event and hadronization processes, $C_{H A D}$ have also been evaluated using HERWIG dijet samples. Figure 5.4 shows the $C_{H A D}$ corrections obtained from HERWIG together with the ones from PYTHIATune $\mathrm{A}$ in all the rapidity regions. The difference between PYTHIA-Tune A and HERWIG is considered as the systematic uncertainty. The determination of $C_{H A D}$ from PYTHIA-Tune A are taken as the nominal one because PYTHIA-Tune A reproduces the underlying event contribution better than HERWIG as it is shown in appendix B. 

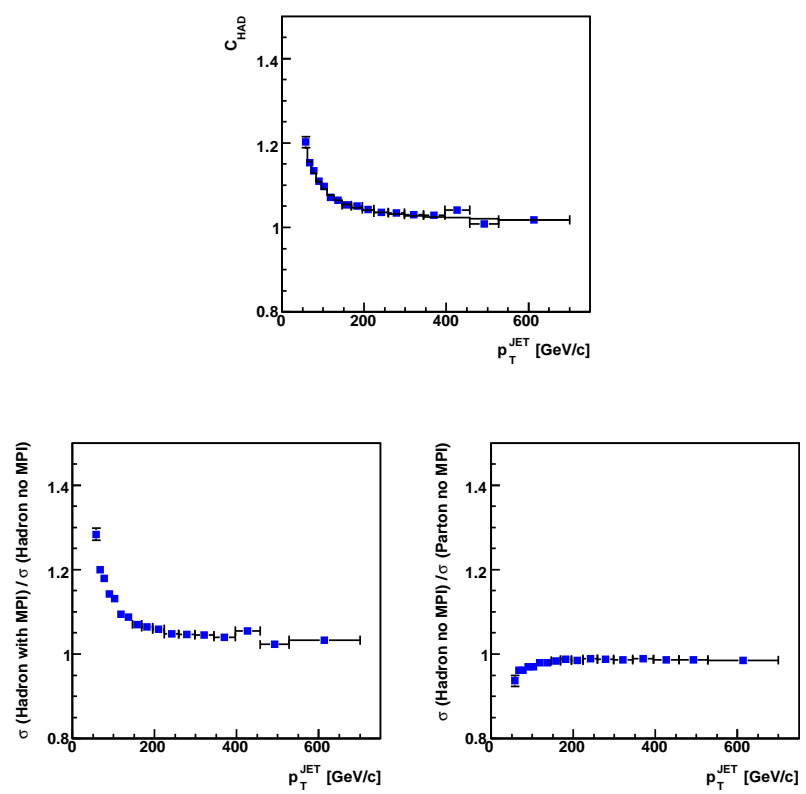

Figure 5.3: Global Underlying Event/Hadronization correction (top) and its factorization into the two contributions:

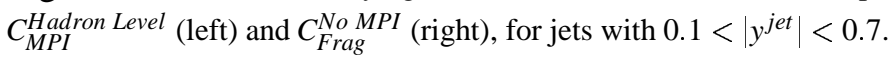

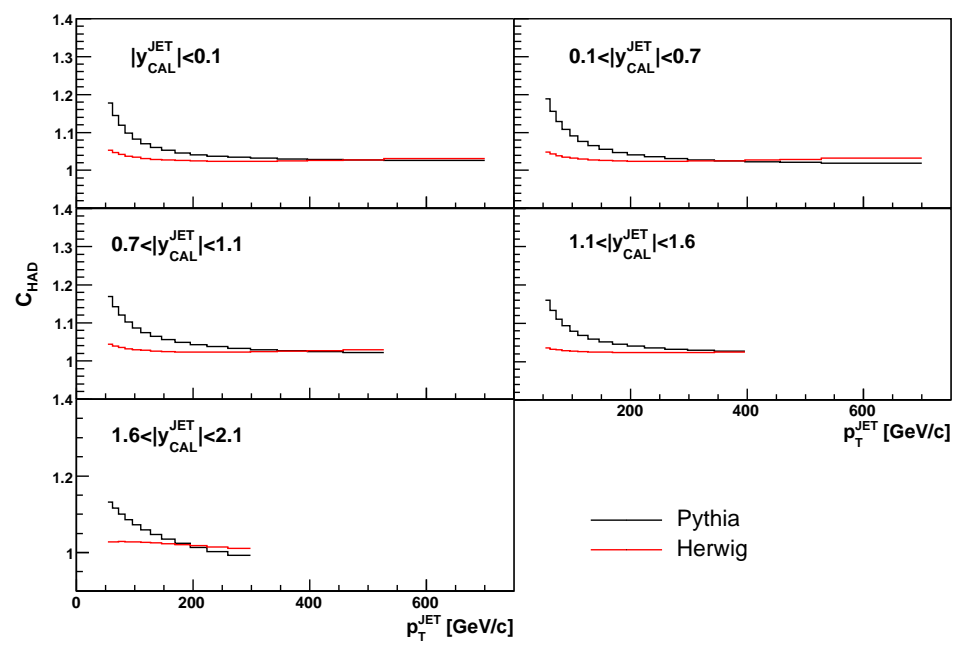

Figure 5.4: Parton to hadron level corrections as obtained from PYTHIA-Tune A (black) and HERWIG (red) for jets in the different rapidity regions. 


\subsection{Inclusive Jet Production results}

The measurements refer to hadron level jets using the $k_{T}$ algorithm with $\mathrm{D}=0.7$ for jets with $p_{T}^{\text {jet }}>$ $54 \mathrm{GeV} / \mathrm{c}$ and in 5 rapidity regions up to $\left|y^{\text {jet }}\right|=2.1$. Figure 5.5 and tables C.6 to C. 10 show the measured inclusive jet cross sections as a function of $p_{T}^{\text {jet }}$. For presentation, the different cross sections are scaled by a given factor. The cross sections decrease over more than seven orders of magnitude as $p_{T}^{\text {jet }}$ increases. The measurements are compared to pQCD NLO predictions, where the theoretical calculations have been properly corrected to account for non-perturbative effects, shown in figure 5.6.

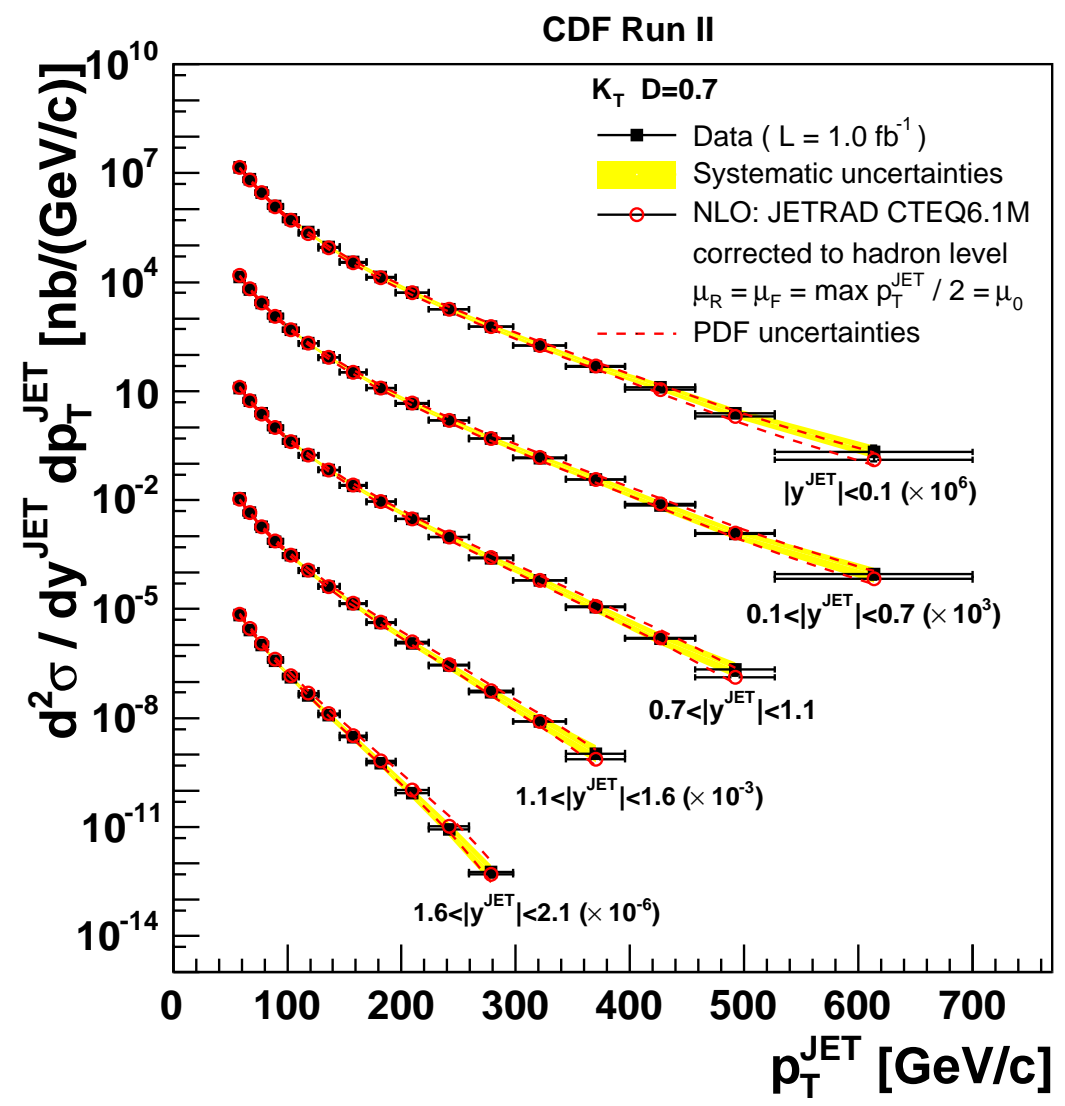

Figure 5.5: Inclusive jet cross sections measured using the $k_{T}$ algorithm with $\mathrm{D}=0.7$ for jets with $p_{T}^{\text {jet }}>54 \mathrm{GeV} / \mathrm{c}$ in five rapidity regions up to $\left|y^{\text {jet }}\right|=2.1$. The black squares represent the measured cross sections and the shaded bands indicate the total systematic uncertainty on the data. The measurements are compared to pQCD NLO calculations. The dashed lines represent the PDFs uncertainties on the theoretical predictions. The $\pm 5.8 \%$ normalization uncertainty coming from the quoted integrated luminosity is not included in the figure. 


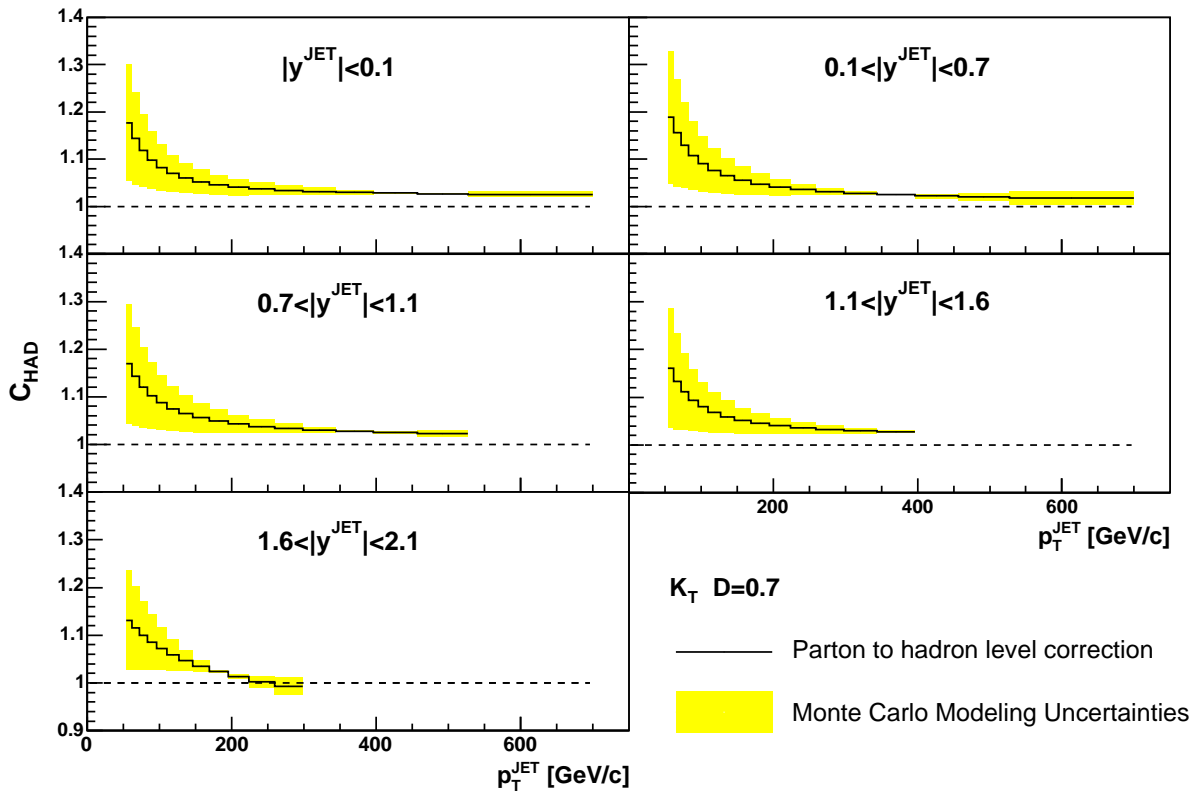

Figure 5.6: Parton to hadron level corrections applied to the NLO calculations to correct for underlying event and hadronization contribution in the different $\left|y^{\text {jet }}\right|$ regions. The shaded bands represent the systematic uncertainty coming from the MC modeling. 
The ratios between the measurements and the QCD predictions, presented in figure 5.7, show a good agreement over all $p_{T}^{\text {jet }}$ ranges in all rapidity regions. Additionally, the figure shows the effect of using MRST2004 PDFs instead of CTEQ6.1M and the results of changing the scales by a factor of 2. Values significantly smaller than $\mu_{0}$ are not considered because give unstable NLO results. In the most forward region, the already smaller uncertainties in the data compared to that in the NLO pQCD calculations show that the measurements will contribute to a better knowledge of the parton distributions inside the proton.

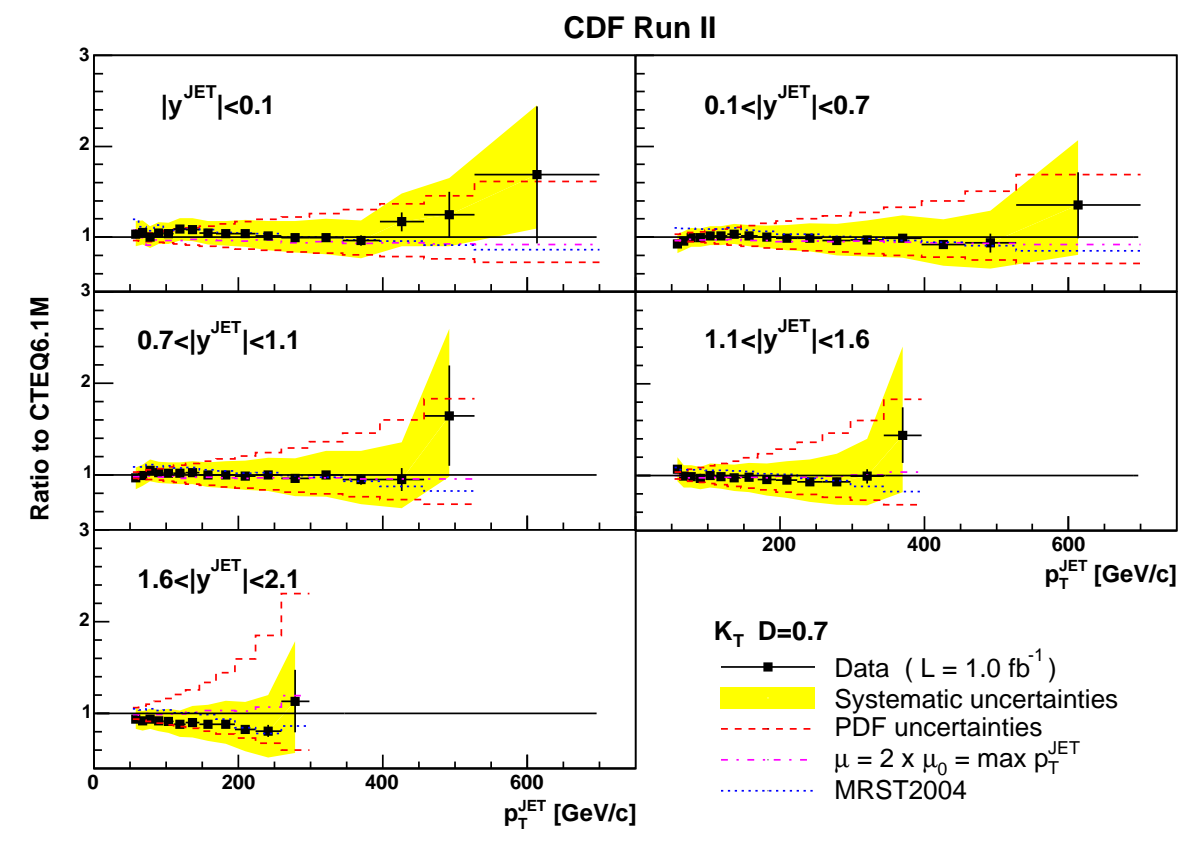

Figure 5.7: Comparison between the measurements and the pQCD calculations. The dots are the ratio Data/Theory using CTEQ6.1M PDFs. The shaded bands indicate the total systematic uncertainty on the data and the dashed lines represent the PDFs uncertainties on the theoretical predictions. The dotted lines present the ration of MRST2004 and CTEQ6.1M predictions. The dotted-dashed lines show the ratio of the theoretical calculations with $2 \mu_{0}$ and $\mu_{0}$. 
For central jets, $0.1<\left|y^{j e t}\right|<0.7$, the measurements are repeated using a D parameter equal to 0.5 and 1.0. As D increases, the average size of the jet in $\eta \times \phi$ space increases, and the measurement becomes more sensitive to underlying event contributions. In this case the pile-up correction, $\varepsilon$, per additional primary vertex are $1.18 \pm 0.12$ and $3.31 \pm 0.47 \mathrm{GeV} / \mathrm{C}$ for $\mathrm{D}=0.5$ and $\mathrm{D}=1.0$, respectively. Figure 5.8 and tables C.11 to C.14 present the measurements. The good agreement still observed between the measured cross sections and the NLO pQCD predictions indicates that the soft contributions are well under control. In this case the corrections applied to the pQCD predictions at low $p_{T}^{\text {jet }}$ are $C_{H A D}=1.1$ for $\mathrm{D}=0.5$ and $C_{H A D}=1.4$ for $\mathrm{D}=1.0$.

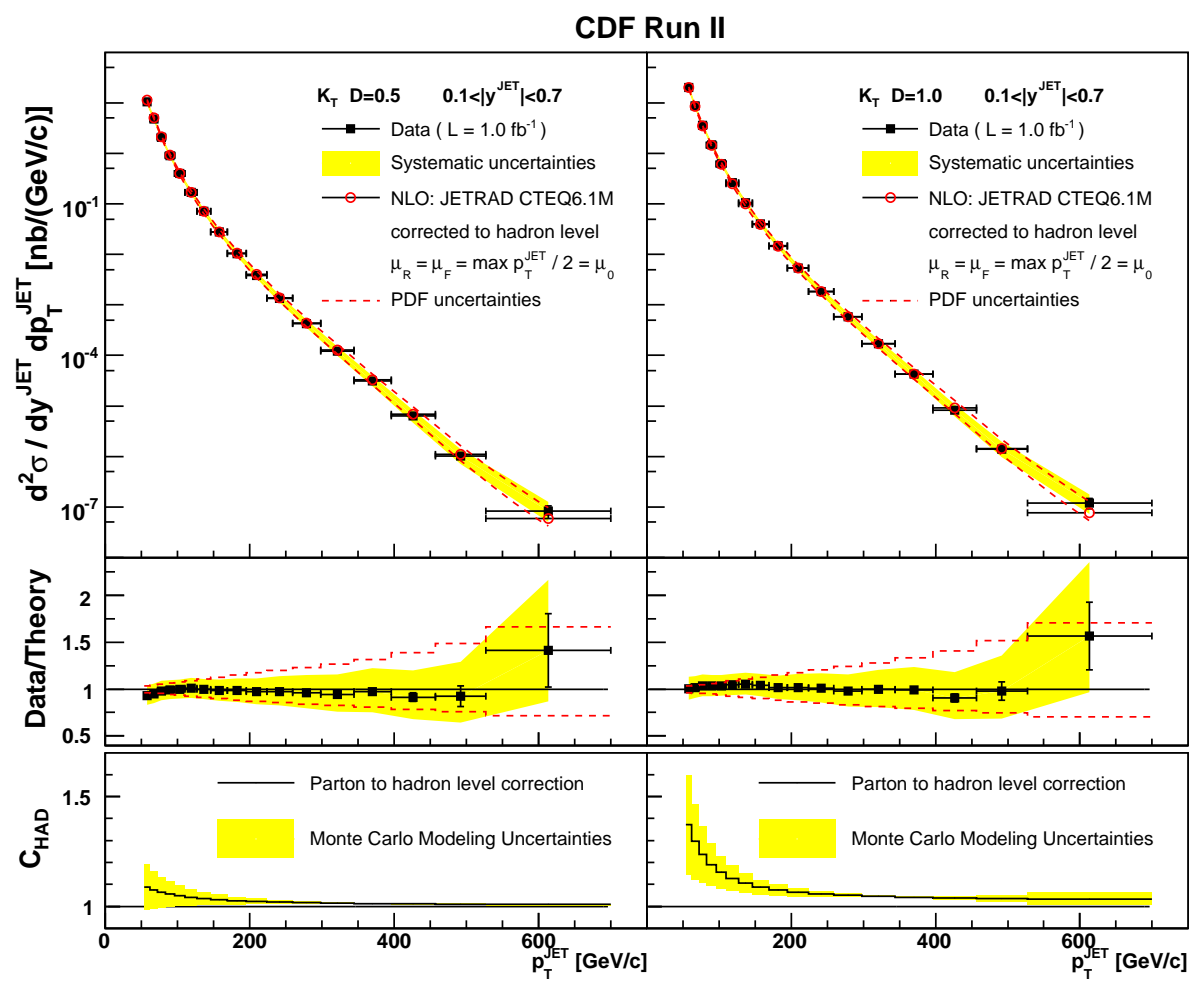

Figure 5.8: Inclusive jet cross sections measured using the $k_{T}$ algorithm with $\mathrm{D}=0.5$ (left) and $\mathrm{D}=1.0$ (right) for jets with $p_{T}^{\text {jet }}>54 \mathrm{GeV} / \mathrm{c}$ and $0.1<\left|y^{\text {jet }}\right|<0.7$. The black squares represent the measured cross sections and the shaded bands indicate the total systematic uncertainty on the data. The measurements are compared to pQCD NLO calculations. The dashed lines represent the PDFs uncertainties on the theoretical predictions. The bottom plots show the parton to hadron level corrections applied to the NLO calculations to correct for underlying event and hadronization effects. The shaded bands represent the systematic uncertainty coming from the MC modeling. 
The results with $\mathrm{D}=1.0$ can be compared with the ones presented by $\mathrm{D} 0$ collaboration during Run I, measurements using the $k_{T}$ algorithm with $\mathrm{D}=1.0$. The $\mathrm{D} 0$ results [61] showed a disagreement of about $40 \%$ with NLO pQCD at low $p_{T}^{\text {jet }}$. The non-perturbative effects could explained those results. 


\section{Chapter 6}

\section{Summary and Conclusions}

Results on inclusive jet production in proton-antiproton collisions at $\sqrt{s}=1.96 \mathrm{TeV}$, based on 1 $\mathrm{fb}^{-1}$ of CDF Run II data, have been presented in this $\mathrm{PhD}$. Thesis. These inclusive measurements constitute a stringent test of pQCD over more than 8 orders of magnitude in cross section and probe distances down to $10^{-19} \mathrm{~m}$. The measurements have been performed using the $k_{T}$ algorithm in a wide kinematic range, for jets with $p_{T}^{j e t}>54 \mathrm{GeV} / \mathrm{c}$ and in the rapidity region $\left|y^{j e t}\right|<2.1$. The measured cross sections have been compared to NLO pQCD calculations, properly corrected to take into account non-perturbative effects.

The measurements are in a good agreement with NLO pQCD calculations. In particular, for central jets and at high $p_{T}^{j e t}$ no deviation with respect to the theory is found, meaning that there is not signal for new physics. In the most forward region, the total systematic uncertainty on the data is smaller than that on the theoretical calculations. Therefore, these new results will contribute to a better understanding of the gluon PDFs at high $\mathrm{x}$ in the proton.

The measurements using different values of the $\mathrm{D}$ parameter have allowed to evaluate the understanding of the non-perturbative effects, important at low $p_{T}^{\text {jet }}$. As D increases, the average size of the jet in $\eta \times \phi$ space increases, and the measurement becomes more sensitive to underlying event contributions. The good agreement observed between the measured cross sections and the NLO pQCD predictions, even with the D parameter set to 1 , indicates that the soft contributions are 
well under control, and validates the use of the $k_{T}$ algorithm in a hadron collider. Therefore, the measurements presented in this work encourage the use of this algorithm in future hadron-hadron experiments, like the LHC. 


\section{Appendix A}

\section{Data Quality Monitoring system}

\section{A.1 Introduction}

The CDF experiment has implemented a system to check the data quality of all the detector components while data is being taken (online) and after the production process (offline). Based on the information obtained with these online and offline monitoring systems, various lists of Good Runs are defined and provided to the different physics groups.

\section{A.2 Online DQM system}

The Online DQM system is part of the CDF online monitoring [62]. The base of the online monitoring is constituted by 10 different monitoring programs, which are in the CDF jargon called consumers. These consumers are permanently running in parallel in the CDF control room and receive, through the Consumer Server Logger (CSL), a copy of a subset of the events that have passed the Level 3. The consumers fill diagnostic histograms, which show the time development of certain quantities, and perform periodical statistical test of the bin contents of those histograms. The 10 monitoring programs are described below.

- YMon: During a physics run YMon receives Minimum Bias events. It makes plots of the following quantities: Occupancies (\% hits per channel per event), average energy per channel and energy distributions. These quantities allow to monitor the rates and distributions of each detector looking for bad channels, cards and power supplies. 
- TrigMon: It monitors various trigger quantities in each trigger bank, format in which the trigger information is stored, looking for hot/failed channels, trigger bits fired, bunch counter mismatches, photomultiplier spikes, etc.

- XMon: It is the responsible to monitor the cross section for each Level 1, Level 2, and Level 3 triggers. With this purpose, it measures cross sections versus instantaneous luminosity and fits them, creating a list with all triggers that have a non-expected behavior during the last 10-15 minutes.

- LumMon: It is an online consumer that monitors the performance of the CLC and the online luminosity measurements obtained with this detector. Moreover, based on the time difference measured between East and West CLC modules, LumMon gives a measurement of the position of the interaction.

- Stage0: It is a special class of online consumer which determines calibration constants critical for production and then writes them to the calibration Data Base.

- BeamMon: The purpose of BeamMon is to find the beamline and determine some properties of the beam spot.

- ObjectMon: Its purpose is to check Level 3 reconstructed objects like jets, electrons, photon and muon candidates and tracks.

- SiliMon: SiliMon produces histograms, hit maps and silicon tracks, with the aim to study the silicon performance efficiency as a function of time and to spot online running problems.

- SVXMon: It is the silicon monitoring consumer used for the online and offline diagnostics of the CDF silicon tracker. For each silicon strip, SVXMon accumulates the number of hits and the first four moments of the pulse height distribution to create plots of occupancies, average pulse heights, distribution shapes, etc... with various detector granularity.

- SVTSPYMon: The source of data analyzed by SPYMon is not the usual data stream out of the CSL, but that recorded by the SVT boards exclusively to monitor the integrity of the SVT system in real time. In this respect, SVTSPYMon collects a large number of tracks in a short period to detect possible SVT hardware faults, fit the beam profile and find SVXII strip noise, etc. 
All the consumers have a HistoDisplay Graphic User Interface (GUI), that allows to see the different consumers outputs. Based on some of these online monitors outputs, a check list is developed to characterize online the quality of the different systems during the run. Shift crew is responsible to go through this list and, at the end of the run, to fill in a table with different bits, set to 1 or 0 , if the system in that run is determined to be good or bad, respectively. This information is included in the Data Base.

\section{A.2.1 Check list}

The systems checked online are the following, where the name of the bits in the Data Base are included in parenthesis: Luminosity counters (CLC), Trigger (L1, L2, L3, SVT), Calorimeters (CAL, CCAL, PCAL), ShowerMax detectors (SMX, CSMX, PSMX), Tracking and Time of flight systems (COT, TOF), Muon system (CMU, CMP, CSP, CMX, CSX, IMU, ISU) and Silicon detectors (SVX, ISL, L00). The checks for every system, which are $90 \%$ based on the YMon consumer, are described below.

- All consumers are running and all the detectors have HV on.

- CLC: Check the occupancy plots in order to verify the good performing of the detector (plots are not empty) and look if the beam position is inside limits. If everything is as expected the CLC bit is set to 1 .

- COT: Check the occupancy plots to look for possible failures in the COT electronics (timeto-digital conversor). The bit is set to 0 if more than 8 cells with 0 occupancy are found.

- TOF: Check the occupancy plots to be sure that there is not too many dead channels. The detector is considered to be working fine if there is less than 4 dead channels.

- Calorimeter system: Check the occupancy plots to monitor dead channels (PMT failure) or hot channels. The detector is determined to be bad if there is any new dead channel or if there are more than 12 consecutive hot channels. In the Data Base, the online status of the different detectors are grouped in three different bits: CCAL (set as $\mathrm{AND}^{1}$ of CEM, CHA and WHA status), PCAL (set as AND of PEM and PHA status) and CAL (set as AND of CCAL and PCAL status).

\footnotetext{
${ }^{1}$ The logical AND operation compares different bits and if they are all "1", then the result is "1", otherwise, the result is " 0 "
} 
- Shower Max detectors: Check the occupancy plots to monitor consecutive dead or hot channels. For each detector the criteria changes slightly. In this way, the CES, CPR, PES, PPR, and CCR detectors are marked bad if the number of consecutive dead (hot) channels are bigger than 32(32), 32(32), 20(32), 20(32) and 10(10) respectively. In the same way to the calorimeter system, the detectors are grouped in three different bits: CSMX (set as AND of CES, CPR and CCR status), PSMX (set as AND of PES and PPR status) and SMX (set as AND of CSMX and PSMX status).

- Muon System: Check the occupancy plots to monitor new dead and noisy channels (PMT failure). CMU is considered OK if the number of dead channels are less than 16 . In the case of the CMP, CSP and CSX detectors the condition is not to find more than 4 contiguous dead channels. For the CMX, the criteria is more strict, and the bit is set to 0 if any new dead channel is found. Finally the IMU system (BMU, BSU, TSU) is considered to be working properly if the number of new dead or noisy channels is $\leq 5$.

- Trigger: Check that the trigger monitoring plots agree with templates provided to the shift crew. Moreover, check if the rate of SVX data corruption errors is $<1 \%$, otherwise, the L3 status bit is set to 0 .

The responsibility of the Consumer Operator (CO), one of the shift crew, is to make entries in a detector check list by going over the systems criteria after 30 minutes of the run starting, repeating at least every 2 hours, and at the end of the run. Once the run has finished, the SiCo (Scientific Coordinator) introduces the $\mathrm{CO}$ check list results (data quality online bits) in the Data Base, being the responsible for marking good/bad every run.

\section{A.2.2 DQM Monitor}

Installed in the control room, and running in parallel with the consumers, there is a DQM Monitor (DQMon). It is an "early warning system" for data quality in events currently being read out of detector. At some level, it is an automated version of the CO, thus the DQMon resumes and monitors the status of all the monitors and the same histograms to be checked by the $\mathrm{CO}$ approximately every 10 seconds. 
Figure A.1 shows the DQMon Panel. Based on the Run Control Color code (red, yellow, blue and green), DQMon displays the status of all the monitors and detector components. In addition the panel shows the information produced by PhysMon about the last five physics runs ${ }^{2}$. In case that any of the subsystem buttons turned red, sound alarm is triggered and an alarm pop window appears. The information about all the subsystem histograms is available, on this way the histograms in red, which are the ones that triggered the alarm, can be identified. The DQM monitor also allows to disables/enables the different systems. At the end of each run, DQMon produces a $\log$ file with all the online bits set to 1 or 0 and recommends to mark the run as GOOD or BAD. It is important to note that this DQMon log file is only a suggestion about the online bits setting, however the SiCo can take it as a reference.

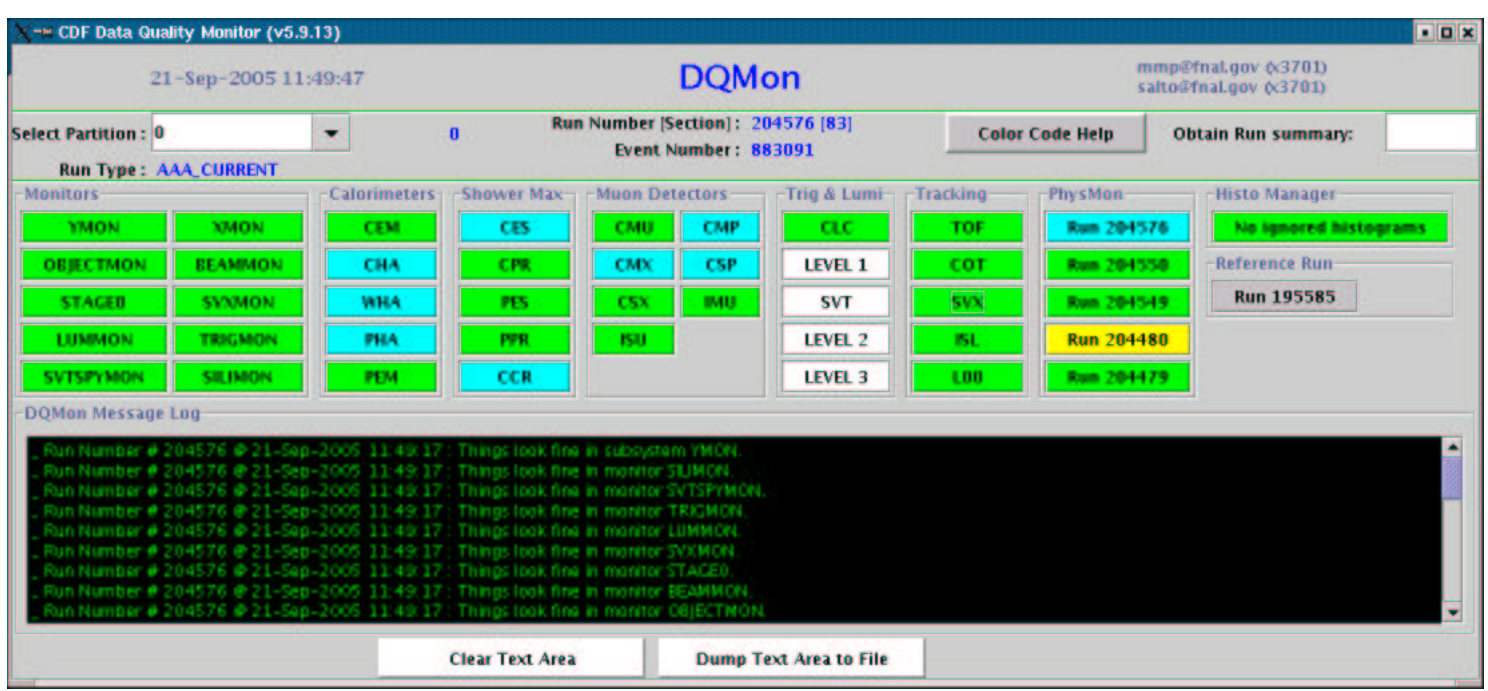

Figure A.1: DQMon panel installed in the control room that displays the status of all the monitors and detector components based on the same criteria than the CO check list.

\footnotetext{
${ }^{2}$ PhyMon is a monitor that processes runs, file by file, and looks at things like electrons, muons, photons, Z's, W's, J/Psi's, and trigger efficiencies. Based on that objects PhysMon declares the run good or bad.
} 
The online DQM system is complemented with an offline monitoring system [63]. Like the online DQM, the offline system uses histograms produced during the offline reprocessing (Production+Validation) of the data, where the latest calibration and alignment constants are used, to characterize offline the quality of detectors and the run.

Finally, based on the online and offline bits introduced in the Data Base for each run, different Good Run Lists [64] are provided to the different physics groups taking into account the detectors used in their analysis. In this way, the lists can be divided in three different categories: QCD group (2 lists), Top/ElectroWeak/Exotic group (18 lists) and B physics group (2 list). 


\section{Appendix B}

\section{Jet Shapes Studies}

\section{B.1 Jet Shapes measurements}

The study of the jet shape is sensitive to the underlying event. The shape of the jet is dominated by multi-gluon emission from the primary parton and it also constitutes a test of the parton shower models and their implementation in the MC programs. Figure B.1 illustrates the integrated jet shape definition $\Psi(r)$, that is the average fraction of the jet transverse momentum that lies inside a cone of radius $r$ concentric to the jet cone. Mathematically, it can be expressed as:

$$
\Psi(r)=\frac{1}{N_{j e t}} \sum \frac{P_{T}(0, r)}{P_{T}(0, R)}, \quad 0 \leq r \leq R
$$

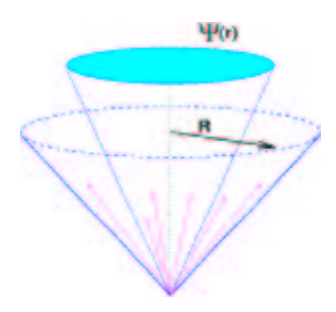

Figure B.1: Illustration of the integrated jet shape definition. 
Figure B.2 presents the CDF Run II measured integrated jet shape for jets defined using the Midpoint algorithm with a cone size $\mathrm{R}=0.7$ [35]. The measurements have been done for jets with $p_{T}^{\text {jet }}$ in the region $37 \mathrm{GeV} / \mathrm{c}<p_{T}^{\text {jet }}<380 \mathrm{GeV} / \mathrm{c}$ and with $0.1<\left|y^{j e t}\right|<0.7$. The measurements have been compared to the prediction from PYTHIA-Tune A and HERWIG MCs. In addition, two different PYTHIA samples have been used with default parameters, with and without multiple parton interaction, in order to study the importance of a proper modeling of soft-gluon radiation. The figure presents the jet shape measurements in two different ways. First, the integrated jet shape for jets in the region $37 \mathrm{GeV} / \mathrm{c}<p_{T}^{j e t}<45 \mathrm{GeV} / \mathrm{c}$ as a function of the fraction $\mathrm{r} / \mathrm{R}$. And second, for a fixed radius $r=0.3$, the average fraction of the jet transverse momentum outside $r=0.3$ as a function of $p_{T}^{\text {jet }}$.
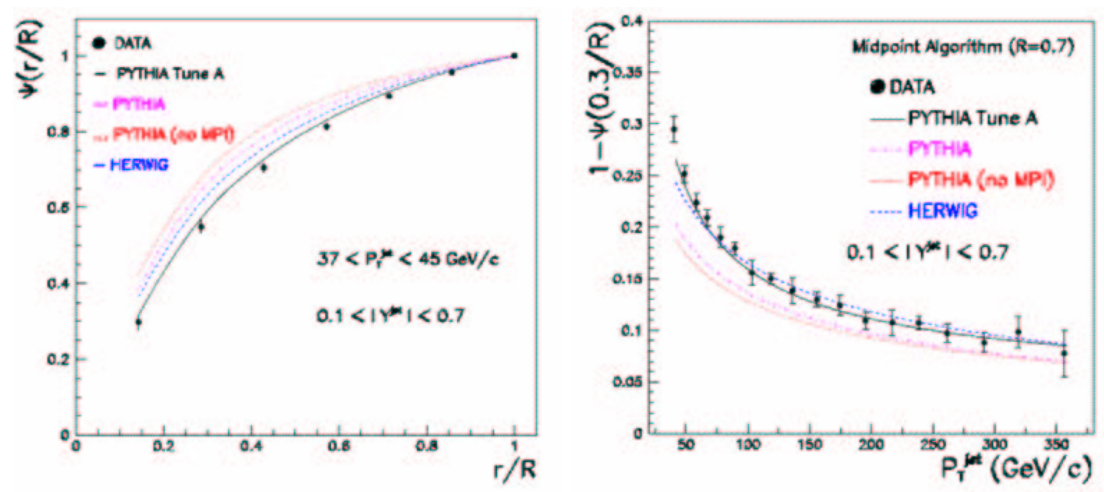

Figure B.2: Measured integrated jet shape for jets with $0.1<\left|y^{j e t}\right|<0.7$ compared to different MC predictions: PYTHIA-Tune A (solid line), PYTHIA (dashed-dotted lines), PYTHIA-(no MPI) (dotted lines) and HERWIG (dashed lines). On the left the integrated jet shape for jets in the $p_{T}^{\text {jet }}$ range $37 \mathrm{GeV} / \mathrm{c}<p_{T}^{\text {jet }}<47$ as a function of the ratio $\mathrm{r} / \mathrm{R}$. On the right, the average fraction of the jet transverse momentum outside $\mathrm{r}=0.3$ as a function of $p_{T}^{\text {jet }}$. Errors bars indicates the statistical and systematic uncertainties added in quadrature.

The measurements show that both give a reasonable prediction, even thought PYTHIA-Tune A predictions describe all of the data well while HERWIG produces too narrow jets at low $p_{T}^{\text {jet }}$. The comparison between PYTHIA and PYTHIA (no MPI) indicates that the contribution from the multiple interactions on the jet shapes is relatively small but relevant at low $p_{T}^{\text {jet }}$. 


\section{Appendix C}

\section{Results Tables}

\begin{tabular}{|c|c|c|c|c|c|c|c|c|}
\hline \multicolumn{6}{|c|}{ systematic uncertainties $(\%)\left(\mid y^{j e t}\right.$} & \multicolumn{3}{|l|}{$<0.1)$} \\
\hline$p_{T}^{j e t}[\mathrm{GeV} / c]$ & jet energy scale & $\begin{array}{l}\beta_{D} \\
\text { (a) }\end{array}$ & $\begin{array}{l}T A / \beta \\
\text { (b) }\end{array}$ & $\begin{array}{l}{ }^{\prime}{ }^{2} \\
\text { (c) }\end{array}$ & resolution & unfolding & $p_{T}^{\text {jet }}$-spectra & $\bar{\varepsilon}$ \\
\hline $54-62$ & $\begin{array}{ll}+10.3 \\
-9.3\end{array}$ & $\begin{array}{l}+1.4 \\
-2.1\end{array}$ & - & - & $\begin{array}{l}+3.8 \\
-3.0\end{array}$ & \pm 8.2 & \pm 1.5 & $\begin{array}{l}+1.8 \\
-1.7\end{array}$ \\
\hline $62-72$ & $\begin{array}{l}+9.9 \\
-9.4\end{array}$ & $\begin{array}{l}+1.7 \\
-2.1\end{array}$ & - & - & $\begin{array}{l}+2.8 \\
-3.0\end{array}$ & \pm 7.1 & \pm 1.4 & $\begin{array}{l}+1.6 \\
-1.5\end{array}$ \\
\hline $72-83$ & $\begin{array}{l}+9.6 \\
-9.4\end{array}$ & $\begin{array}{l}+1.9 \\
-2.1\end{array}$ & - & - & $\begin{array}{l}+2.9 \\
-3.0\end{array}$ & \pm 6.2 & \pm 1.3 & $\begin{array}{l}+1.4 \\
-1.3\end{array}$ \\
\hline $83-96$ & $\begin{array}{l}+9.4 \\
-9.5\end{array}$ & $\begin{array}{l}+2.1 \\
-2.2\end{array}$ & - & - & $\begin{array}{l}+2.9 \\
-2.9\end{array}$ & \pm 5.4 & \pm 1.1 & $\begin{array}{l}+1.3 \\
-1.1\end{array}$ \\
\hline $96-110$ & $\begin{array}{l}+9.5 \\
-9.6\end{array}$ & $\begin{array}{l}+2.3 \\
-2.2\end{array}$ & - & - & $\begin{array}{l}+2.9 \\
-2.9\end{array}$ & \pm 4.7 & \pm 1.0 & $\begin{array}{l}+1.1 \\
-1.0\end{array}$ \\
\hline $110-127$ & $\begin{array}{r}+9.8 \\
-9.8\end{array}$ & $\begin{array}{l}+2.5 \\
-2.3\end{array}$ & - & - & $\begin{array}{l}+3.0 \\
-2.9\end{array}$ & \pm 4.2 & \pm 0.9 & $\begin{array}{l}+1.0 \\
{ }_{-0.9}\end{array}$ \\
\hline $127-146$ & $\begin{array}{l}+10.4 \\
-10.2\end{array}$ & $\begin{array}{l}+2.7 \\
-2.4\end{array}$ & - & - & $\begin{array}{l}+3.1 \\
-2.9\end{array}$ & \pm 3.7 & \pm 0.8 & $\begin{array}{l}+0.9 \\
-0.8\end{array}$ \\
\hline $146-169$ & $\begin{array}{l}+11.2 \\
-10.8\end{array}$ & $\begin{array}{l}+2.8 \\
-2.6\end{array}$ & - & - & $\begin{array}{l}+3.1 \\
-3.0\end{array}$ & \pm 3.2 & \pm 0.6 & $\begin{array}{l}+0.8 \\
-0.8\end{array}$ \\
\hline $169-195$ & $\begin{array}{l}+12.4 \\
-11.6\end{array}$ & $\begin{array}{l}+2.9 \\
-2.7\end{array}$ & - & - & $\begin{array}{l}+3.3 \\
-3.0\end{array}$ & \pm 2.8 & \pm 0.5 & $\begin{array}{l}+0.7 \\
-0.7\end{array}$ \\
\hline $195-224$ & $\begin{array}{l}+13.9 \\
-12.8\end{array}$ & $\begin{array}{l}+3.0 \\
-2.9\end{array}$ & - & - & $\begin{array}{l}+3.4 \\
-3.2\end{array}$ & \pm 2.5 & \pm 0.4 & $\begin{array}{l}+0.6 \\
-0.7\end{array}$ \\
\hline $224-259$ & $\begin{array}{l}+15.5 \\
-14.3\end{array}$ & $\begin{array}{l}+3.1 \\
-3.1\end{array}$ & - & - & $\begin{array}{l}+3.7 \\
-3.4\end{array}$ & \pm 2.2 & \pm 0.3 & $\begin{array}{l}+0.6 \\
-0.6\end{array}$ \\
\hline $259-298$ & $\begin{array}{l}+17.4 \\
-159\end{array}$ & $\begin{array}{r}+3.3 \\
+3.4\end{array}$ & - & - & $\begin{array}{l}+4.0 \\
-36\end{array}$ & \pm 2.0 & \pm 0.4 & $\begin{array}{l}+0.5 \\
-0.5\end{array}$ \\
\hline $298-344$ & $\begin{array}{r}+19.5 \\
-17.4\end{array}$ & $\begin{array}{l}+3.4 \\
+3.6 \\
-3.7\end{array}$ & - & - & $\begin{array}{l}+4.0 \\
+4.0\end{array}$ & \pm 1.8 & \pm 0.6 & $\begin{array}{l}+0.5 \\
-0.6\end{array}$ \\
\hline $344-396$ & $\begin{array}{l}+22.1 \\
-19.1\end{array}$ & $\begin{array}{l}+4.0 \\
-4.0\end{array}$ & - & - & $\begin{array}{l}+4.8 \\
-4.5\end{array}$ & \pm 1.6 & \pm 1.0 & $\begin{array}{l}+0.4 \\
-0.5\end{array}$ \\
\hline $396-457$ & $\begin{array}{l}+25.7 \\
-21.6\end{array}$ & $\begin{array}{l}+4.6 \\
-4.4\end{array}$ & - & - & $\begin{array}{l}+5.4 \\
-5.1\end{array}$ & \pm 1.4 & \pm 1.8 & $\begin{array}{l}+0.4 \\
-0.5\end{array}$ \\
\hline $457-527$ & $\begin{array}{l}+31.3 \\
-26.3\end{array}$ & $\begin{array}{l}+5.3 \\
-5.1\end{array}$ & - & - & $\begin{array}{l}+6.1 \\
-5.9\end{array}$ & \pm 1.3 & \pm 3.1 & $\begin{array}{l}+0.3 \\
-0.5\end{array}$ \\
\hline $527-700$ & $\begin{array}{l}+43.7 \\
{ }_{-32.9}\end{array}$ & $\begin{array}{l}+7.3 \\
-6.7\end{array}$ & - & - & $\begin{array}{l}+7.4 \\
-7.3\end{array}$ & \pm 1.1 & \pm 7.1 & ${ }_{-0.5}^{+0.3}$ \\
\hline
\end{tabular}

Table C.1: Systematic uncertainties (in percentage) on the measured inclusive jet differential cross section as a function of $p_{T}^{j e t}$ for jets in the region $\left|y^{\text {jet }}\right|<0.1$. The different columns follow the discussion in section 4.6. An additional 5.8\% uncertainty on the luminosity is not included. 


\begin{tabular}{|c|c|c|c|c|c|c|c|c|}
\hline \multicolumn{9}{|c|}{ systematic uncertainties $(\%)\left(0.1<\left|y^{j e t}\right|\right.$} \\
\hline$p_{T}^{j e t}[\mathrm{GeV} / c]$ & jet energy scale & \multicolumn{3}{|c|}{$\beta_{D A T A} / \beta_{M C}$} & resolution & unfolding & $p_{T}^{j e t}$-spectra & $\varepsilon$ \\
\hline $54-62$ & +9.5 & - & - & - & +2.2 & \pm 5.3 & \pm 0.6 & +1.6 \\
\hline $62-72$ & $\begin{array}{l}+9.4 \\
-9.1\end{array}$ & - & - & - & $\begin{array}{l}+2.1 \\
-2.4\end{array}$ & \pm 4.7 & \pm 0.6 & $\begin{array}{l}+1.5 \\
-1.4\end{array}$ \\
\hline $72-83$ & +9.4 & - & - & - & +2.1 & \pm 4.1 & \pm 0.5 & +1.3 \\
\hline $83-96$ & $\begin{array}{l}+9.4 \\
-8.9\end{array}$ & - & - & - & $\begin{array}{l}+2.0 \\
-2.3\end{array}$ & \pm 3.7 & \pm 0.5 & $\begin{array}{l}+1.2 \\
-1.1\end{array}$ \\
\hline $96-110$ & +9.6 & - & - & - & +2.0 & \pm 3.3 & \pm 0.5 & +1.1 \\
\hline $110-127$ & +10.0 & - & - & - & +1.9 & \pm 3.0 & \pm 0.5 & $\begin{array}{l}+1.0 \\
-0.9\end{array}$ \\
\hline $127-146$ & +10.6 & - & - & - & +1.9 & \pm 2.7 & \pm 0.5 & $\begin{array}{l}+0.9 \\
-0.8\end{array}$ \\
\hline $146-169$ & $\begin{array}{l}+11.4 \\
-106\end{array}$ & - & - & - & $\begin{array}{l}+1.9 \\
-2.0\end{array}$ & \pm 2.4 & \pm 0.4 & $\begin{array}{l}+0.8 \\
-0.8\end{array}$ \\
\hline $169-195$ & $\begin{array}{l}+12.6 \\
-11.7\end{array}$ & - & - & - & +2.0 & \pm 2.2 & \pm 0.4 & $\begin{array}{l}+0.7 \\
-0.7\end{array}$ \\
\hline $195-224$ & +14.1 & - & - & - & +2.1 & \pm 2.0 & \pm 0.4 & +0.7 \\
\hline $224-259$ & $\begin{array}{l}+16.0 \\
-14.8\end{array}$ & - & - & - & $\begin{array}{l}+2.2 \\
-2.3\end{array}$ & \pm 1.8 & \pm 0.3 & $\begin{array}{l}+0.6 \\
+0.6\end{array}$ \\
\hline $259-298$ & $\begin{array}{r}+18.4 \\
-167\end{array}$ & - & - & - & $\begin{array}{r}+2.5 \\
-25\end{array}$ & \pm 1.7 & \pm 0.3 & +0.6 \\
\hline $298-344$ & $\begin{array}{l}+21.3 \\
-18.9\end{array}$ & - & - & - & $\begin{array}{l}+2.8 \\
-2.9\end{array}$ & \pm 1.6 & \pm 0.3 & $\begin{array}{l}+0.5 \\
-0.6\end{array}$ \\
\hline $344-396$ & +25.1 & - & - & - & +3.4 & \pm 1.5 & \pm 0.5 & +0.5 \\
\hline $396-457$ & $\begin{array}{l}+30.3 \\
-24.7\end{array}$ & - & - & - & $\begin{array}{l}+4.1 \\
-42\end{array}$ & \pm 1.4 & \pm 0.8 & +0.4 \\
\hline $457-527$ & $\begin{array}{l}+37.7 \\
-293\end{array}$ & - & - & - & $\begin{array}{l}+5.1 \\
+5.2\end{array}$ & \pm 1.3 & \pm 1.4 & +0.4 \\
\hline $527-700$ & $\begin{array}{l}+52.3 \\
-39.8\end{array}$ & - & - & - & $\begin{array}{l}+7.3 \\
-7.3\end{array}$ & \pm 1.2 & \pm 3.6 & $\begin{array}{l}+0.4 \\
-0.5\end{array}$ \\
\hline
\end{tabular}

Table C.2: Systematic uncertainties (in percentage) on the measured inclusive jet differential cross section as a function of $p_{T}^{j e t}$ for jets in the region $0.1<\left|y^{j e t}\right|<0.7$. The different columns follow the discussion in section 4.6. An additional $5.8 \%$ uncertainty on the luminosity is not included. 


\begin{tabular}{|c|c|c|c|c|c|c|c|c|}
\hline \multicolumn{9}{|c|}{ systematic uncertainties $(\%)\left(0.7<\left|y^{j e t}\right|<1.1\right)$} \\
\hline$p_{T}^{\text {jet }}[\mathrm{GeV} / c]$ & jet energy scale & \multicolumn{3}{|c|}{$\beta_{D A T A} / \beta_{M C}$} & \multirow[t]{2}{*}{ resolution } & \multirow[t]{2}{*}{ unfolding } & \multirow[t]{2}{*}{$p_{T}^{j e t}$-spectra } & \multirow[t]{2}{*}{$\varepsilon$} \\
\hline & & (a) & (b) & (c) & & & & \\
\hline $54-62$ & $\begin{array}{l}+9.2 \\
-9.9\end{array}$ & $\begin{array}{l}+2.1 \\
-2.3\end{array}$ & - & - & $\begin{array}{l}+4.0 \\
-3.8\end{array}$ & \pm 6.3 & \pm 2.0 & $\begin{array}{l}+1.7 \\
-1.6\end{array}$ \\
\hline $62-72$ & 9.3 & $\begin{array}{l}+2.2 \\
-2.3\end{array}$ & - & - & $\begin{array}{l}+3.8 \\
-3.7\end{array}$ & \pm 5.6 & \pm 1.9 & $\begin{array}{l}+1.5 \\
-1.4\end{array}$ \\
\hline $72-83$ & $\begin{array}{l}+9.2 \\
{ }_{-9.0}\end{array}$ & $\begin{array}{l}+2.3 \\
-2.3\end{array}$ & - & - & $\begin{array}{l}+3.7 \\
-3.5\end{array}$ & \pm 4.9 & \pm 1.8 & $\begin{array}{l}+1.3 \\
-1.3\end{array}$ \\
\hline $83-96$ & $\begin{array}{l}+9.5 \\
+9.0\end{array}$ & $\begin{array}{l}+2.3 \\
+2.3\end{array}$ & - & - & $\begin{array}{l}+3.5 \\
+3.4\end{array}$ & \pm 4.4 & \pm 1.8 & $\begin{array}{l}+1.2 \\
-1.2\end{array}$ \\
\hline $96-110$ & $\begin{array}{l}+9.9 \\
-9.3\end{array}$ & $\begin{array}{l}+2.4 \\
+2.4\end{array}$ & - & - & $\begin{array}{l}+3.4 \\
+3.3\end{array}$ & \pm 3.9 & \pm 1.7 & $\begin{array}{l}+1.1 \\
-1.1\end{array}$ \\
\hline $110-127$ & $\begin{array}{l}-10.6 \\
+9.8\end{array}$ & $\begin{array}{l}-2.4 \\
+2.5\end{array}$ & - & - & $\begin{array}{l}+3.3 \\
+3.3 \\
-3.2\end{array}$ & \pm 3.5 & \pm 1.7 & $\begin{array}{l}-1.1 \\
+1.0\end{array}$ \\
\hline $127-146$ & $\begin{array}{l}-9.8 \\
+11.5 \\
-107\end{array}$ & $\begin{array}{l}-2.3 \\
+2.6\end{array}$ & - & - & $\begin{array}{l}-3.2 \\
+3.3 \\
-3.1\end{array}$ & \pm 3.2 & \pm 1.7 & $\begin{array}{l}-1.0 \\
+0.9 \\
-0.9\end{array}$ \\
\hline $146-169$ & $\begin{array}{l}+12.6 \\
+11.7\end{array}$ & $\begin{array}{l}+2.8 \\
+2.7\end{array}$ & - & - & $\begin{array}{l}+3.1 \\
+3.2\end{array}$ & \pm 2.8 & \pm 1.6 & $\begin{array}{l}+0.8 \\
-0.8\end{array}$ \\
\hline $169-195$ & $\begin{array}{l}+14.1 \\
-13.0\end{array}$ & $\begin{array}{l}+3.0 \\
-2.9\end{array}$ & - & - & $\begin{array}{l}+3.4 \\
-3.3\end{array}$ & \pm 2.6 & \pm 1.6 & $\begin{array}{l}+0.8 \\
{ }_{-0.8}\end{array}$ \\
\hline $195-224$ & $\begin{array}{l}+15.9 \\
-14.6\end{array}$ & $\begin{array}{l}+3.3 \\
-3.2\end{array}$ & - & - & $\begin{array}{l}+3.7 \\
-3.5\end{array}$ & \pm 2.3 & \pm 1.7 & $\begin{array}{l}+0.7 \\
-0.7\end{array}$ \\
\hline $224-259$ & $\begin{array}{l}+18.1 \\
+165\end{array}$ & $\begin{array}{r}+3.8 \\
+36\end{array}$ & - & - & $\begin{array}{r}+4.1 \\
-3.9\end{array}$ & \pm 2.1 & \pm 1.8 & $\begin{array}{l}+0.7 \\
-0.7\end{array}$ \\
\hline $259-298$ & $\begin{array}{l}-10.0 \\
+21.0 \\
-19.2\end{array}$ & $\begin{array}{l}-5.0 \\
+4.4\end{array}$ & - & - & $\begin{array}{l}-4.7 \\
+4.5\end{array}$ & \pm 2.0 & \pm 2.1 & $\begin{array}{l}-0.6 \\
+0.6\end{array}$ \\
\hline $298-344$ & $\begin{array}{l}+25.2 \\
-22.7\end{array}$ & $\begin{array}{l}+5.0 \\
-4.8\end{array}$ & - & - & $\begin{array}{l}+5.6 \\
-5.3\end{array}$ & \pm 1.8 & \pm 2.4 & $\begin{array}{l}+0.6 \\
-0.6\end{array}$ \\
\hline $344-396$ & $\begin{array}{l}-22.1 \\
+31.5 \\
-26.9\end{array}$ & $\begin{array}{l}-4.8 \\
+5.9\end{array}$ & - & - & $\begin{array}{l}-5.3 \\
+6.8 \\
-6.4\end{array}$ & \pm 1.7 & \pm 3.0 & $\begin{array}{l}-0.0 \\
+0.6\end{array}$ \\
\hline $396-457$ & $\begin{array}{l}-40.3 \\
+31.0\end{array}$ & $\begin{array}{l}-3.0 \\
+7.2 \\
-6.6\end{array}$ & - & - & $\begin{array}{l}-8.4 \\
+8.3 \\
-7.7\end{array}$ & \pm 1.6 & \pm 3.8 & $\begin{array}{l}-0.0 \\
+0.5\end{array}$ \\
\hline $457-527$ & $\begin{array}{l}-51.0 \\
+58.4 \\
-38.3\end{array}$ & $\begin{array}{l}-0.0 \\
+10.4 \\
-7.7\end{array}$ & - & - & ${ }_{-9.1}^{-10.0}$ & \pm 1.5 & \pm 5.0 & $\begin{array}{l}-0.3 \\
+0.5\end{array}$ \\
\hline
\end{tabular}

Table C.3: Systematic uncertainties (in percentage) on the measured inclusive jet differential cross section as a function of $p_{T}^{\text {jet }}$ for jets in the region $0.7<\left|y^{\text {jet }}\right|<1$. 1 . The different columns follow the discussion in section 4.6. An additional $5.8 \%$ uncertainty on the luminosity is not included. 


\begin{tabular}{|c|c|c|c|c|c|c|c|c|}
\hline \multicolumn{9}{|c|}{ systematic uncertainties $(\%)\left(1.1<\left|y^{\text {jet }}\right|\right.$} \\
\hline$p_{T}^{j e t}[\mathrm{GeV} / c]$ & jet energy scale & \multicolumn{3}{|c|}{$\beta_{D A T A} / \beta_{M C}$} & resolution & unfolding & $p_{T}^{j e t}$-spectra & $\varepsilon$ \\
\hline & & (a) & (b) & (c) & & & & \\
\hline $54-62$ & $\begin{array}{l}+9.4 \\
-8.6\end{array}$ & $\begin{array}{l}+2.6 \\
-2.4\end{array}$ & - & $\begin{array}{l}+0.0 \\
-3.0\end{array}$ & $\begin{array}{l}+2.9 \\
-3.1\end{array}$ & \pm 6.7 & \pm 1.3 & $\begin{array}{l}+1.8 \\
-1.8\end{array}$ \\
\hline $62-72$ & +9.5 & +2.5 & - & +0.0 & +2.9 & \pm 6.4 & \pm 1.1 & +1.6 \\
\hline $72-83$ & +9.8 & +2.5 & - & +0.0 & +2.9 & +61 & +09 & $\begin{array}{l}+1.4 \\
\end{array}$ \\
\hline $12-83$ & -9.3 & -2.5 & & -3.0 & -2.9 & 工0.1 & \pm 0.9 & -1.3 \\
\hline $83-96$ & $\begin{array}{l}+10.2 \\
-9.8\end{array}$ & $\begin{array}{l}+2.5 \\
-2.6\end{array}$ & - & $\begin{array}{l}+0.0 \\
-3.0\end{array}$ & $\begin{array}{l}+2.9 \\
-2.8\end{array}$ & \pm 5.8 & \pm 0.8 & $\begin{array}{l}+1.3 \\
-1.2\end{array}$ \\
\hline $96-110$ & $\begin{array}{l}+10.9 \\
-105\end{array}$ & $\begin{array}{l}+2.6 \\
-2.6\end{array}$ & - & $\begin{array}{l}+0.0 \\
-3.0\end{array}$ & $\begin{array}{l}+3.0 \\
-2.9\end{array}$ & \pm 5.6 & \pm 0.6 & $\begin{array}{l}+1.2 \\
-1.1\end{array}$ \\
\hline $110-127$ & $\begin{array}{r}+11.7 \\
+11.4\end{array}$ & +2.7 & - & +0.0 & +3.1 & \pm 5.4 & \pm 0.4 & +1.1 \\
\hline $127-146$ & +12.8 & +2.9 & - & +0.0 & +3.4 & +52 & +03 & +1.1 \\
\hline & -12.6 & -3.0 & - & -3.0 & $\begin{array}{l}-3.2 \\
+3.2\end{array}$ & & & -1.0 \\
\hline $146-169$ & $\begin{array}{l}+14.5 \\
-14.2\end{array}$ & $\begin{array}{l}+3.3 \\
-3.3\end{array}$ & - & $\begin{array}{l}+0.0 \\
-3.0\end{array}$ & $\begin{array}{l}+3.8 \\
-3.6\end{array}$ & \pm 5.0 & \pm 0.1 & $\begin{array}{l}+1.0 \\
-0.9\end{array}$ \\
\hline $169-195$ & $\begin{array}{l}+16.9 \\
-162\end{array}$ & $\begin{array}{l}+3.8 \\
-3.7\end{array}$ & - & $\begin{array}{l}+0.0 \\
-3.0\end{array}$ & $\begin{array}{l}+4.3 \\
-4.2\end{array}$ & \pm 4.8 & \pm 0.1 & $\begin{array}{l}+1.0 \\
-0.9\end{array}$ \\
\hline $195-224$ & $\begin{array}{l}+20.3 \\
-186\end{array}$ & +4.4 & $\begin{array}{l}+0.7 \\
-0.9\end{array}$ & +0.0 & +5.1 & \pm 4.7 & \pm 0.2 & $\begin{array}{l}+0.9 \\
+0.9\end{array}$ \\
\hline $224-259$ & $\begin{array}{l}-18.0 \\
+24.7\end{array}$ & $\begin{array}{l}-4.2 \\
+5.2\end{array}$ & $\begin{array}{l}-0.9 \\
+2.6\end{array}$ & $\begin{array}{l}-5.0 \\
+0.0\end{array}$ & $\begin{array}{l}-3.0 \\
+6.2\end{array}$ & \pm 4.6 & \pm 0.4 & $\begin{array}{l}-0.9 \\
+0.9\end{array}$ \\
\hline & $\begin{array}{l}-21.2 \\
+29.9\end{array}$ & $\begin{array}{l}-5.0 \\
+6.2\end{array}$ & $\begin{array}{l}-2.4 \\
+6.3\end{array}$ & $\begin{array}{l}-3.0 \\
+0.0\end{array}$ & $\begin{array}{l}-6.1 \\
+7.8\end{array}$ & 5 & & \\
\hline $259-298$ & -24.1 & -5.9 & -4.5 & -3.0 & -7.3 & \pm 4.4 & \pm 0.8 & -0.9 \\
\hline $298-344$ & $\begin{array}{l}+37.2 \\
-28.6\end{array}$ & $\begin{array}{l}+7.3 \\
-7.1\end{array}$ & $\begin{array}{l}+12.6 \\
-7.5\end{array}$ & $\begin{array}{l}+0.0 \\
-3.0\end{array}$ & $\begin{array}{l}+9.8 \\
-8.5\end{array}$ & \pm 4.3 & \pm 1.6 & $\begin{array}{l}+0.9 \\
-0.9\end{array}$ \\
\hline $344-396$ & +61.2 & $\begin{array}{l}+8.7 \\
-8.3\end{array}$ & $\begin{array}{l}+22.7 \\
-117\end{array}$ & +0.0 & $\begin{array}{l}+12.4 \\
-94\end{array}$ & \pm 4.2 & \pm 2.8 & $\begin{array}{l}+0.9 \\
+0.9\end{array}$ \\
\hline
\end{tabular}

Table C.4: Systematic uncertainties (in percentage) on the measured inclusive jet differential cross section as a function of $p_{T}^{\text {jet }}$ for jets in the region $1.1<\left|y^{\text {jet }}\right|<1.6$. The different columns follow the discussion in section 4.6. An additional $5.8 \%$ uncertainty on the luminosity is not included. 


\begin{tabular}{|c|c|c|c|c|c|c|c|c|}
\hline \multicolumn{9}{|c|}{ systematic uncertainties $(\%)\left(1.6<\left|y^{j e t}\right|<2.1\right)$} \\
\hline$p_{T}^{\text {jet }}[\mathrm{GeV} / c]$ & jet energy scale & \multicolumn{3}{|c|}{$\beta_{D A T A} / \beta_{M C}$} & \multirow[t]{2}{*}{ resolution } & \multirow[t]{2}{*}{ unfolding } & \multirow[t]{2}{*}{$p_{T}^{\text {jet }}$-spectra } & \multirow[t]{2}{*}{$\varepsilon$} \\
\hline & & (a) & (b) & (c) & & & & \\
\hline $54-62$ & $\begin{array}{l}+11.6 \\
-10.3\end{array}$ & ${ }_{-2.1}^{+2.3}$ & - & - & $\begin{array}{l}+1.7 \\
-1.6\end{array}$ & \pm 3.2 & \pm 1.0 & ${ }_{-2.0}^{+2.1}$ \\
\hline $62-72$ & $\begin{array}{l}+10.9 \\
+10.9\end{array}$ & +2.4 & - & - & $\begin{array}{l}-1.0 \\
+1.7\end{array}$ & \pm 3.3 & \pm 0.8 & ${ }_{-1.8}^{+1.8}$ \\
\hline $72-83$ & $\begin{array}{l}+11.0 \\
+10.3\end{array}$ & $\begin{array}{l}-2.6 \\
-2.6\end{array}$ & - & - & $\begin{array}{l}-1.1 \\
+1.7\end{array}$ & \pm 3.4 & \pm 0.6 & $\begin{array}{l}-1.0 \\
+1.7\end{array}$ \\
\hline $83-96$ & $\begin{array}{l}+12.0 \\
-11.1\end{array}$ & $\begin{array}{l}+2.8 \\
-2.9\end{array}$ & - & - & $\begin{array}{l}+1.5 \\
-1.8\end{array}$ & \pm 3.5 & \pm 0.4 & $\begin{array}{l}+1.6 \\
{ }_{-1.6}\end{array}$ \\
\hline $96-110$ & $\begin{array}{l}+13.7 \\
-12.5\end{array}$ & $\begin{array}{l}+3.2 \\
-3.2\end{array}$ & - & - & $\begin{array}{l}+1.5 \\
-1.8\end{array}$ & \pm 3.6 & \pm 0.3 & $\begin{array}{l}+1.5 \\
+1.5\end{array}$ \\
\hline $110-127$ & $\begin{array}{l}+16.2 \\
+14.4\end{array}$ & $\begin{array}{r}+3.7 \\
-3.5\end{array}$ & - & - & $\begin{array}{l}+1.6 \\
+1.9\end{array}$ & \pm 3.7 & \pm 0.2 & ${ }_{-1.4}^{+1.4}$ \\
\hline $127-146$ & $\begin{array}{l}-1.4 \\
+19.2 \\
-169\end{array}$ & $\begin{array}{l}-4.3 \\
+4.0\end{array}$ & - & - & $\begin{array}{l}-1.9 \\
+1.8 \\
-2.0\end{array}$ & \pm 3.7 & \pm 0.1 & $\begin{array}{l}-1.4 \\
+1.4 \\
-1.4\end{array}$ \\
\hline $146-169$ & $\begin{array}{l}-10.9 \\
+22.8 \\
-19.8\end{array}$ & $\begin{array}{l}-4.0 \\
+5.0 \\
-4.6\end{array}$ & - & - & $\begin{array}{l}-2.0 \\
+2.1\end{array}$ & \pm 3.8 & \pm 0.2 & $\begin{array}{l}-1.4 \\
{ }_{-1.3}^{+1.4}\end{array}$ \\
\hline $169-195$ & $\begin{array}{l}-1.8 \\
+27.7 \\
-23.0\end{array}$ & $\begin{array}{l}-4.0 \\
+6.0 \\
-5.4\end{array}$ & ${ }_{-09}^{+1.3}$ & - & $\begin{array}{l}-2.1 \\
+2.5\end{array}$ & \pm 3.8 & \pm 0.5 & $\begin{array}{l}-1.3 \\
+1.4\end{array}$ \\
\hline $195-224$ & $\begin{array}{r}-24.9 \\
+26.9 \\
-26.7\end{array}$ & $\begin{array}{l}-7.4 \\
+6.0 \\
-6.4\end{array}$ & $\begin{array}{r}-0.9 \\
+5.3\end{array}$ & - & $\begin{array}{l}-3.0 \\
+3.0\end{array}$ & \pm 3.8 & \pm 1.1 & +1.4 \\
\hline $224-259$ & $\begin{array}{l}-46.1 \\
+46.0\end{array}$ & $\begin{array}{l}-6.4 \\
+8.1\end{array}$ & $\begin{array}{r}-5.6 \\
+11.0\end{array}$ & - & $\begin{array}{l}-2.7 \\
+3.5\end{array}$ & \pm 3.8 & +2.1 & $\begin{array}{l}-1.3 \\
+1.4\end{array}$ \\
\hline $259-298$ & $\begin{array}{l}-32.4 \\
+52.9 \\
+5\end{array}$ & $\begin{array}{l}-8.0 \\
+9.1\end{array}$ & $\begin{array}{l}-11.1 \\
+19.1\end{array}$ & - & $\begin{array}{r}-3.3 \\
+3.9\end{array}$ & \pm 3.8 & \pm 3.7 & $\begin{array}{l}-1.3 \\
+1.4\end{array}$ \\
\hline
\end{tabular}

Table C.5: Systematic uncertainties (in percentage) on the measured inclusive jet differential cross section as a function of $p_{T}^{j e t}$ for jets in the region $1.6<\left|y^{j e t}\right|<2.1$. The different columns follow the discussion in section 4.6. An additional 5.8\% uncertainty on the luminosity is not included. 


\begin{tabular}{|c|c|c|}
\hline \multicolumn{3}{|c|}{$\frac{d^{2} \sigma}{d p_{T}^{j e t} d y^{j e t}}\left(\left|y^{j e t}\right|<0.1\right)$} \\
\hline$p_{T}^{\text {jet }}[\mathrm{GeV} / c]$ & $\bar{\sigma} \pm($ stat. $) \pm($ sys. $)[\mathrm{nb} /(\mathrm{GeV} / \mathrm{c})]$ & $\mathrm{C}_{\text {HAD }}$ parton $\rightarrow$ hadron \\
\hline $54-62$ & $\left(14.5 \pm 0.5_{-1.9}^{+2.0}\right) \times 10^{0}$ & $1.177 \pm 0.124$ \\
\hline $62-72$ & $\left(6.68 \pm 0.08_{-0.84}^{+0.85}\right) \times 10^{0}$ & $1.144 \pm 0.097$ \\
\hline $72-83$ & $\left(2.87 \pm 0.05_{-0.34}^{+0.35}\right) \times 10^{0}$ & $1.119 \pm 0.077$ \\
\hline $83-96$ & $\left(1.24 \pm 0.02_{-0.14}^{+0.14}\right) \times 10^{0}$ & $1.098 \pm 0.061$ \\
\hline $96-110$ & $\left(5.31 \pm 0.11_{-0.61}^{+0.60}\right) \times 10^{-1}$ & $1.083 \pm 0.049$ \\
\hline $110-127$ & $\left(2.33 \pm 0.06_{-0.26}^{+0.27}\right) \times 10^{-1}$ & $1.070 \pm 0.039$ \\
\hline $127-146$ & $\left(9.36 \pm 0.12_{-1.08}^{+1.10}\right) \times 10^{-2}$ & $1.060 \pm 0.032$ \\
\hline $146-169$ & $\left(3.63 \pm 0.06_{-0.43}^{+0.45}\right) \times 10^{-2}$ & $1.052 \pm 0.026$ \\
\hline $169-195$ & $\left(1.39 \pm 0.01_{-0.18}^{+0.19}\right) \times 10^{-2}$ & $1.046 \pm 0.021$ \\
\hline $195-224$ & $\left(5.22 \pm 0.06_{-0.72}^{+0.77}\right) \times 10^{-3}$ & $1.041 \pm 0.017$ \\
\hline $224-259$ & $\left(1.79 \pm 0.03_{-0.27}^{+0.29}\right) \times 10^{-3}$ & $1.037 \pm 0.013$ \\
\hline $259-298$ & $\left(5.92 \pm 0.11_{-1.00}^{+1.08}\right) \times 10^{-4}$ & $1.034 \pm 0.010$ \\
\hline $298-344$ & $\left(1.78 \pm 0.06_{-0.33}^{+0.36}\right) \times 10^{-4}$ & $1.032 \pm 0.007$ \\
\hline $344-396$ & $\left(4.68 \pm 0.28_{-0.94}^{+1.08}\right) \times 10^{-5}$ & $1.030 \pm 0.005$ \\
\hline $396-457$ & $\left(1.29 \pm 0.12_{-0.29}^{+0.34}\right) \times 10^{-5}$ & $1.028 \pm 0.002$ \\
\hline $457-527$ & $\left(2.47 \pm 0.50_{-0.68}^{+0.80}\right) \times 10^{-6}$ & $1.027 \pm 0.001$ \\
\hline $527-700$ & $\left(2.13 \pm 0.95_{-0.75}^{+0.97}\right) \times 10^{-7}$ & $1.026 \pm 0.006$ \\
\hline
\end{tabular}

Table C.6: Measured inclusive jet differential cross section as a function of $p_{T}^{\text {jet }}$ for jets in the region $\left|y^{\text {jet }}\right|<0.1$. An additional 5.8\% uncertainty on the luminosity is not included. The parton-to-hadron correction factors, $\mathrm{C}_{\mathrm{HAD}}\left(\mathrm{p}_{\mathrm{T}}^{\mathrm{jet}}, \mathrm{y}^{\mathrm{jet}}\right)$, are applied to the $\mathrm{pQCD}$ predictions. 


\begin{tabular}{|c|c|c|}
\hline \multicolumn{3}{|c|}{$\frac{d^{2} \sigma}{d p_{T}^{j e t} d y^{j e t}}\left(0.1<\left|y^{\text {jet }}\right|<0.7\right)$} \\
\hline 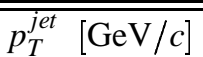 & 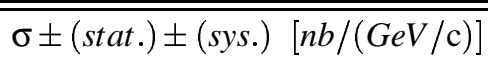 & $\mathrm{C}_{\mathrm{HAD}}$ parton $\rightarrow$ hadron \\
\hline $54-62$ & $\left(14.0 \pm 0.20_{-1.6}^{+1.6}\right) \times 10^{0}$ & $1.188 \pm 0.140$ \\
\hline $62-72$ & $\left(6.14 \pm 0.12_{-0.65}^{+0.66}\right) \times 10^{0}$ & $1.156 \pm 0.113$ \\
\hline $72-83$ & $\left(2.69 \pm 0.02_{-0.27}^{+0.29}\right) \times 10^{0}$ & $1.129 \pm 0.091$ \\
\hline $83-96$ & $\left(1.14 \pm 0.01_{-0.11}^{+0.12}\right) \times 10^{0}$ & $1.108 \pm 0.073$ \\
\hline $96-110$ & $\left(4.90 \pm 0.04_{-0.48}^{+0.51}\right) \times 10^{-1}$ & $1.090 \pm 0.059$ \\
\hline $110-127$ & $\left(2.08 \pm 0.02_{-0.21}^{+0.22}\right) \times 10^{-1}$ & $1.076 \pm 0.047$ \\
\hline $127-146$ & $\left(8.51 \pm 0.04_{-0.89}^{+0.95}\right) \times 10^{-2}$ & $1.065 \pm 0.038$ \\
\hline $146-169$ & $\left(3.33 \pm 0.02_{-0.37}^{+0.40}\right) \times 10^{-2}$ & $1.055 \pm 0.029$ \\
\hline $169-195$ & $\left(1.23 \pm 0.01_{-0.15}^{+0.16}\right) \times 10^{-2}$ & $1.047 \pm 0.023$ \\
\hline $195-224$ & $\left(4.53 \pm 0.02_{-0.61}^{+0.65}\right) \times 10^{-3}$ & $1.041 \pm 0.017$ \\
\hline $224-259$ & $\left(1.57 \pm 0.01_{-0.24}^{+0.26}\right) \times 10^{-3}$ & $1.036 \pm 0.012$ \\
\hline $259-298$ & $\left(4.87 \pm 0.06_{-0.83}^{+0.91}\right) \times 10^{-4}$ & $1.031 \pm 0.007$ \\
\hline $298-344$ & $\left(1.43 \pm 0.02_{-0.27}^{+0.31}\right) \times 10^{-4}$ & $1.028 \pm 0.003$ \\
\hline $344-396$ & $\left(3.69 \pm 0.10_{-0.80}^{+0.94}\right) \times 10^{-5}$ & $1.025 \pm 0.001$ \\
\hline $396-457$ & $\left(7.18 \pm 0.34_{-1.80}^{+2.20}\right) \times 10^{-6}$ & $1.023 \pm 0.004$ \\
\hline $457-527$ & $\left(1.16 \pm 0.13_{-0.35}^{+0.44}\right) \times 10^{-6}$ & $1.021 \pm 0.008$ \\
\hline $527-700$ & $\left(8.97 \pm 2.40_{-3.64}^{+4.75}\right) \times 10^{-8}$ & $1.018 \pm 0.014$ \\
\hline
\end{tabular}

Table C.7: Measured inclusive jet differential cross section as a function of $p_{T}^{\text {jet }}$ for jets in the region $0.1<\left|y^{j e t}\right|<0.7$.An additional $5.8 \%$ uncertainty on the luminosity is not included. The parton-to-hadron correction factors, $\mathrm{C}_{\mathrm{HAD}}\left(\mathrm{p}_{\mathrm{T}}^{\mathrm{jet}}, \mathrm{y}^{\mathrm{jet}}\right)$, are applied to the $\mathrm{pQCD}$ predictions. 


\begin{tabular}{|c|c|c|}
\hline \multicolumn{3}{|c|}{$\frac{d^{2} \sigma}{d p_{T}^{j e t} d y^{j e t}}\left(0.7<\left|y^{j e t}\right|<1.1\right)$} \\
\hline $\bar{p} p_{T}^{\text {jet }}[\mathrm{GeV} / c]$ & 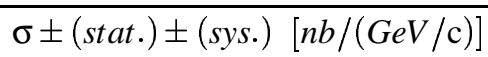 & $\mathrm{C}_{\mathrm{HAD}}$ parton $\rightarrow$ hadron \\
\hline $54-62$ & $\left(12.3 \pm 0.2_{-1.5}^{+1.5}\right) \times 10^{0}$ & $1.169 \pm 0.125$ \\
\hline $62-72$ & $\left(5.48 \pm 0.14_{-0.65}^{+0.65}\right) \times 10^{0}$ & $1.143 \pm 0.103$ \\
\hline $72-83$ & $\left(2.40 \pm 0.02_{-0.27}^{+0.28}\right) \times 10^{0}$ & $1.120 \pm 0.085$ \\
\hline $83-96$ & $\left(1.00 \pm 0.01_{-0.11}^{+0.15}\right) \times 10^{0}$ & $1.102 \pm 0.070$ \\
\hline $96-110$ & $\left(4.15 \pm 0.05_{-0.46}^{+0.48}\right) \times 10^{-1}$ & $1.087 \pm 0.057$ \\
\hline $110-127$ & $\left(1.73 \pm 0.03_{-0.20}^{+0.21}\right) \times 10^{-1}$ & $1.075 \pm 0.047$ \\
\hline $127-146$ & $\left(6.83 \pm 0.05_{-0.82}^{+0.87}\right) \times 10^{-2}$ & $1.064 \pm 0.038$ \\
\hline $146-169$ & $\left(2.52 \pm 0.03_{-0.33}^{+0.35}\right) \times 10^{-2}$ & $1.056 \pm 0.031$ \\
\hline $169-195$ & $\left(8.95 \pm 0.06_{-1.26}^{+1.36}\right) \times 10^{-3}$ & $1.048 \pm 0.024$ \\
\hline $195-224$ & $\left(3.04 \pm 0.02_{-0.47}^{+0.51}\right) \times 10^{-3}$ & $1.042 \pm 0.019$ \\
\hline $224-259$ & $\left(9.52 \pm 0.11_{-1.68}^{+1.82}\right) \times 10^{-4}$ & $1.037 \pm 0.014$ \\
\hline $259-298$ & $\left(2.53 \pm 0.05_{-0.51}^{+0.56}\right) \times 10^{-4}$ & $1.033 \pm 0.009$ \\
\hline $298-344$ & $\left(6.18 \pm 0.17_{-1.49}^{+1.64}\right) \times 10^{-5}$ & $1.030 \pm 0.005$ \\
\hline $344-396$ & $\left(1.11 \pm 0.07_{-0.31}^{+0.36}\right) \times 10^{-5}$ & $1.027 \pm 0.001$ \\
\hline $396-457$ & $\left(1.53 \pm 0.20_{-0.50}^{+0.65}\right) \times 10^{-6}$ & $1.025 \pm 0.003$ \\
\hline $457-527$ & $\left(2.17 \pm 0.72_{-0.88}^{+1.25}\right) \times 10^{-7}$ & $1.023 \pm 0.007$ \\
\hline
\end{tabular}

Table C.8: Measured inclusive jet differential cross section as a function of $p_{T}^{\text {jet }}$ for jets in the region $0.7<\left|y^{\text {jet }}\right|<1.1$. An additional $5.8 \%$ uncertainty on the luminosity is not included. The parton-to-hadron correction factors, $\mathrm{C}_{\mathrm{HAD}}\left(\mathrm{p}_{\mathrm{T}}^{\mathrm{jet}}, \mathrm{y}^{\mathrm{jet}}\right)$, are applied to the $\mathrm{pQCD}$ predictions. 


\begin{tabular}{|c|c|c|}
\hline \multicolumn{3}{|c|}{$\frac{d^{2} \sigma}{d p_{T}^{j e t} d y^{j e t}}\left(1.1<\left|y^{j e t}\right|<1.6\right)$} \\
\hline$p_{T}^{\text {jet }}[\mathrm{GeV} / c]$ & $\sigma \pm($ stat. $) \pm($ sys. $)[\mathrm{nb} /(\mathrm{GeV} / \mathrm{c})]$ & $\mathrm{C}_{\mathrm{HAD}}$ parton $\rightarrow$ hadron \\
\hline $54-62$ & $\left(11.0 \pm 0.3_{-1.3}^{+1.4}\right) \times 10^{0}$ & $1.160 \pm 0.125$ \\
\hline $62-72$ & $\left(4.40 \pm 0.15_{-0.53}^{+0.54}\right) \times 10^{0}$ & $1.133 \pm 0.101$ \\
\hline $72-83$ & $\left(1.82 \pm 0.06_{-0.22}^{+0.22}\right) \times 10^{0}$ & $1.111 \pm 0.081$ \\
\hline $83-96$ & $\left(7.22 \pm 0.37_{-0.90}^{+0.90}\right) \times 10^{-1}$ & $1.094 \pm 0.065$ \\
\hline $96-110$ & $\left(2.98 \pm 0.05_{-0.38}^{+0.38}\right) \times 10^{-1}$ & $1.080 \pm 0.052$ \\
\hline $110-127$ & $\left(1.14 \pm 0.03_{-0.15}^{+0.15}\right) \times 10^{-1}$ & $1.068 \pm 0.042$ \\
\hline $127-146$ & $\left(4.10 \pm 0.04_{-0.60}^{+0.60}\right) \times 10^{-2}$ & $1.059 \pm 0.034$ \\
\hline $146-169$ & $\left(1.39 \pm 0.02_{-0.23}^{+0.22}\right) \times 10^{-2}$ & $1.051 \pm 0.027$ \\
\hline $169-195$ & $\left(4.19 \pm 0.04_{-0.76}^{+0.78}\right) \times 10^{-3}$ & $1.045 \pm 0.021$ \\
\hline $195-224$ & $\left(1.15 \pm 0.02_{-0.24}^{+0.25}\right) \times 10^{-3}$ & $1.040 \pm 0.016$ \\
\hline $224-259$ & $\left(2.73 \pm 0.09_{-0.64}^{+0.73}\right) \times 10^{-4}$ & $1.036 \pm 0.012$ \\
\hline $259-298$ & $\left(5.18 \pm 0.23_{-1.39}^{+1.68}\right) \times 10^{-5}$ & $1.033 \pm 0.009$ \\
\hline $298-344$ & $\left(7.99 \pm 0.61_{-2.56}^{+3.31}\right) \times 10^{-6}$ & $1.030 \pm 0.006$ \\
\hline $344-396$ & $\left(1.05 \pm 0.22_{-0.45}^{+0.71}\right) \times 10^{-6}$ & $1.028 \pm 0.003$ \\
\hline
\end{tabular}

Table C.9: Measured inclusive jet differential cross section as a function of $p_{T}^{\text {jet }}$ for jets in the region $1.1<\left|y^{j e t}\right|<1.6$. An additional $5.8 \%$ uncertainty on the luminosity is not included. The parton-to-hadron correction factors, $\mathrm{C}_{\mathrm{HAD}}\left(\mathrm{p}_{\mathrm{T}}^{\text {jet }}\right)$, are applied to the $\mathrm{pQCD}$ predictions. 


\begin{tabular}{|c|c|c|}
\hline \multicolumn{3}{|c|}{$\frac{d^{2} \sigma}{d p_{T}^{j e t} d y^{j e t}}\left(1.6<\left|y^{j e t}\right|<2.1\right)$} \\
\hline$p_{T}^{\text {jet }}[\mathrm{GeV} / c]$ & $\bar{\sigma} \sigma \pm($ stat. $) \pm($ sys. $)[\mathrm{nb} /(\mathrm{GeV} / \mathrm{c})]$ & $\mathrm{C}_{\mathrm{HAD}}$ parton $\rightarrow$ hadron \\
\hline$[\mathrm{GeV} / c]$ & {$[\mathrm{nb} /(\mathrm{GeV} / \mathrm{c})]$} & parton $\rightarrow$ hadron \\
\hline $54-62$ & $\left(6.67 \pm 0.15_{-0.75}^{+0.84}\right) \times 10^{0}$ & $1.132 \pm 0.104$ \\
\hline $62-72$ & $\left(2.68 \pm 0.02_{-0.30}^{+0.32}\right) \times 10^{0}$ & $1.116 \pm 0.087$ \\
\hline $72-83$ & $\left(1.04 \pm 0.01_{-0.12}^{+0.12}\right) \times 10^{0}$ & $1.100 \pm 0.072$ \\
\hline $83-96$ & $\left(3.77 \pm 0.04_{-0.46}^{+0.49}\right) \times 10^{-1}$ & $1.086 \pm 0.058$ \\
\hline $96-110$ & $\left(1.32 \pm 0.02_{-0.18}^{+0.19}\right) \times 10^{-1}$ & $1.072 \pm 0.045$ \\
\hline $110-127$ & $\left(4.18 \pm 0.04_{-0.65}^{+0.72}\right) \times 10^{-2}$ & $1.059 \pm 0.033$ \\
\hline $127-146$ & $\left(1.21 \pm 0.02_{-0.22}^{+0.24}\right) \times 10^{-2}$ & $1.047 \pm 0.022$ \\
\hline $146-169$ & $\left(2.92 \pm 0.04_{-0.61}^{+0.70}\right) \times 10^{-3}$ & $1.035 \pm 0.012$ \\
\hline $169-195$ & $\left(5.74 \pm 0.09_{-1.38}^{+1.65}\right) \times 10^{-4}$ & $1.024 \pm 0.003$ \\
\hline $195-224$ & $\left(8.49 \pm 0.31_{-2.42}^{+3.09}\right) \times 10^{-5}$ & $1.013 \pm 0.005$ \\
\hline $224-259$ & $\left(8.65 \pm 0.63_{-3.08}^{+4.18}\right) \times 10^{-6}$ & $1.003 \pm 0.012$ \\
\hline $259-298$ & $\left(5.67 \pm 1.65_{-2.80}^{+3.25}\right) \times 10^{-7}$ & $0.993 \pm 0.018$ \\
\hline
\end{tabular}

Table C.10: Measured inclusive jet differential cross section as a function of $p_{T}^{\text {jet }}$ for jets in the region $1.6<\left|y^{j e t}\right|<2.1$. An additional $5.8 \%$ uncertainty on the luminosity is not included. The parton-to-hadron correction factors, $\mathrm{C}_{\mathrm{HAD}}\left(\mathrm{p}_{\mathrm{T}}^{\mathrm{jet}}, \mathrm{y}^{\mathrm{jet}}\right)$, are applied to the $\mathrm{pQCD}$ predictions. 


\begin{tabular}{|c|c|c|}
\hline \multicolumn{3}{|c|}{$\frac{d^{2} \sigma}{d p_{T}^{j e t} d y^{j e t}}\left(0.1<\left|y^{j e t}\right|<0.7\right) \quad(D=0.5)$} \\
\hline$p_{T}^{j e t}[\mathrm{GeV} / c]$ & $\sigma \pm($ stat. $) \pm($ sys. $)[\mathrm{nb} /(\mathrm{GeV} / \mathrm{c})]$ & $\mathrm{C}_{\text {HAD }}$ parton $\rightarrow$ hadron \\
\hline $54-62$ & $\left(10.5 \pm 0.2_{-1.1}^{+1.2}\right) \times 10^{0}$ & $1.089 \pm 0.104$ \\
\hline $62-72$ & $\left(4.81 \pm 0.03_{-0.50}^{+0.54}\right) \times 10^{0}$ & $1.076 \pm 0.086$ \\
\hline $72-83$ & $\left(2.09 \pm 0.01_{-0.21}^{+0.23}\right) \times 10^{0}$ & $1.064 \pm 0.070$ \\
\hline $83-96$ & $\left(0.91 \pm 0.01_{-0.09}^{+0.10}\right) \times 10^{0}$ & $1.055 \pm 0.057$ \\
\hline $96-110$ & $\left(3.95 \pm 0.04_{-0.39}^{+0.42}\right) \times 10^{-1}$ & $1.047 \pm 0.047$ \\
\hline $110-127$ & $\left(1.71 \pm 0.02_{-0.17}^{+0.18}\right) \times 10^{-1}$ & $1.041 \pm 0.037$ \\
\hline $127-146$ & $\left(0.71 \pm 0.01_{-0.07}^{+0.08}\right) \times 10^{-1}$ & $1.035 \pm 0.029$ \\
\hline $146-169$ & $\left(2.76 \pm 0.02_{-0.31}^{+0.32}\right) \times 10^{-2}$ & $1.030 \pm 0.023$ \\
\hline $169-195$ & $\left(1.04 \pm 0.01_{-0.13}^{+0.14}\right) \times 10^{-2}$ & $1.026 \pm 0.017$ \\
\hline $195-224$ & $\left(3.87 \pm 0.02_{-0.53}^{+0.57}\right) \times 10^{-3}$ & $1.022 \pm 0.012$ \\
\hline $224-259$ & $\left(1.34 \pm 0.01_{-0.21}^{+0.23}\right) \times 10^{-3}$ & $1.019 \pm 0.008$ \\
\hline $259-298$ & $\left(4.26 \pm 0.04_{-0.74}^{+0.83}\right) \times 10^{-4}$ & $1.017 \pm 0.005$ \\
\hline $298-344$ & $\left(1.22 \pm 0.02_{-0.24}^{+0.28}\right) \times 10^{-4}$ & $1.015 \pm 0.002$ \\
\hline $344-396$ & $\left(3.16 \pm 0.09_{-0.71}^{+0.82}\right) \times 10^{-5}$ & $1.013 \pm 0.001$ \\
\hline $396-457$ & $\left(6.30 \pm 0.32_{-1.63}^{+1.96}\right) \times 10^{-6}$ & $1.011 \pm 0.002$ \\
\hline $457-527$ & $\left(1.01 \pm 0.12_{-0.31}^{+0.40}\right) \times 10^{-6}$ & $1.010 \pm 0.003$ \\
\hline $527-700$ & $\left(0.83 \pm 0.23_{-0.32}^{+0.44}\right) \times 10^{-7}$ & $1.008 \pm 0.005$ \\
\hline
\end{tabular}

Table C.11: Measured inclusive jet differential cross section as a function of $p_{T}^{\text {jet }}$ for jets in the region $0.1<\left|y^{\text {jet }}\right|<0.7$ using $D=0.5$. An additional $5.8 \%$ uncertainty on the luminosity is not included. The parton-tohadron correction factors, $\mathrm{C}_{\mathrm{HAD}}\left(\mathrm{p}_{\mathrm{T}}^{\mathrm{jet}}\right)$, are applied to the $\mathrm{pQCD}$ predictions. 


\begin{tabular}{|c|c|c|}
\hline \multicolumn{3}{|c|}{$\frac{d^{2} \sigma}{d p_{T}^{j e t} d y^{j e t}}\left(0.1<\left|y^{j e t}\right|<0.7\right) \quad(D=1.0)$} \\
\hline 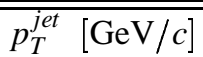 & 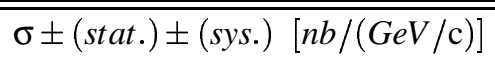 & $\mathrm{C}_{\mathrm{HAD}}$ parton $\rightarrow$ hadron \\
\hline $54-62$ & $\left(20.0 \pm 0.2_{-2.3}^{+2.6}\right) \times 10^{0}$ & $1.372 \pm 0.227$ \\
\hline $62-72$ & $\left(8.65 \pm 0.04_{-1.0}^{+1.1}\right) \times 10^{0}$ & $1.296 \pm 0.171$ \\
\hline $72-83$ & $\left(3.59 \pm 0.02_{-0.39}^{+0.42}\right) \times 10^{0}$ & $1.236 \pm 0.129$ \\
\hline $83-96$ & $\left(1.49 \pm 0.01_{-0.16}^{+0.17}\right) \times 10^{0}$ & $1.190 \pm 0.098$ \\
\hline $96-110$ & $\left(6.27 \pm 0.05_{-0.65}^{+0.70}\right) \times 10^{-1}$ & $1.155 \pm 0.075$ \\
\hline $110-127$ & $\left(2.63 \pm 0.03_{-0.27}^{+0.29}\right) \times 10^{-1}$ & $1.127 \pm 0.057$ \\
\hline $127-146$ & $\left(1.05 \pm 0.01_{-0.11}^{+0.12}\right) \times 10^{-1}$ & $1.105 \pm 0.044$ \\
\hline $146-169$ & $\left(4.04 \pm 0.03_{-0.45}^{+0.48}\right) \times 10^{-2}$ & $1.088 \pm 0.034$ \\
\hline $169-195$ & $\left(1.48 \pm 0.01_{-0.18}^{+0.19}\right) \times 10^{-2}$ & $1.075 \pm 0.026$ \\
\hline $195-224$ & $\left(5.41 \pm 0.02_{-0.73}^{+0.77}\right) \times 10^{-3}$ & $1.065 \pm 0.019$ \\
\hline $224-259$ & $\left(1.86 \pm 0.01_{-0.28}^{+0.30}\right) \times 10^{-3}$ & $1.057 \pm 0.013$ \\
\hline $259-298$ & $\left(5.77 \pm 0.04_{-1.00}^{+1.05}\right) \times 10^{-4}$ & $1.050 \pm 0.008$ \\
\hline $298-344$ & $\left(1.70 \pm 0.02_{-0.32}^{+0.36}\right) \times 10^{-4}$ & $1.045 \pm 0.003$ \\
\hline $344-396$ & $\left(4.26 \pm 0.10_{-0.93}^{+1.05}\right) \times 10^{-5}$ & $1.041 \pm 0.003$ \\
\hline $396-457$ & $\left(8.17 \pm 0.36_{-2.06}^{+2.49}\right) \times 10^{-6}$ & $1.038 \pm 0.009$ \\
\hline $457-527$ & $\left(1.39 \pm 0.14_{-0.42}^{+0.55}\right) \times 10^{-6}$ & $1.036 \pm 0.015$ \\
\hline $527-700$ & $\left(1.19 \pm 0.27_{-0.46}^{+0.60}\right) \times 10^{-7}$ & $1.033 \pm 0.027$ \\
\hline
\end{tabular}

Table C.12: Measured inclusive jet differential cross section as a function of $p_{T}^{j e t}$ for jets in the region $0.1<\left|y^{\text {jet }}\right|<0.7$ using $D=1.0$. An additional $5.8 \%$ uncertainty on the luminosity is not included. The parton-tohadron correction factors, $\mathrm{C}_{\mathrm{HAD}}\left(\mathrm{p}_{\mathrm{T}}^{\text {jet }}\right)$, are applied to the $\mathrm{PQCD}$ predictions. 


\begin{tabular}{|c|c|c|c|c|c|}
\hline \multicolumn{6}{|c|}{ systematic uncertainties $(\%)\left(0.1<\left|y^{\text {jet }}\right|<0.7\right)(D=0.5)$} \\
\hline$p_{T}^{\text {jet }}[\mathrm{GeV} / c]$ & jet energy scale & resolution & unfolding & $p_{T}^{\text {jet }}$-spectra & $\varepsilon$ \\
\hline $54-62$ & $\begin{array}{l}+9.9 \\
-9.2\end{array}$ & $\begin{array}{l}+2.4 \\
-2.3\end{array}$ & \pm 5.4 & \pm 0.6 & $\begin{array}{ll}+0.8 \\
-0.8\end{array}$ \\
\hline $62-72$ & $\begin{array}{l}+9.8 \\
-9.0\end{array}$ & $\begin{array}{l}+2.4 \\
-2.2\end{array}$ & \pm 4.8 & \pm 0.6 & $\begin{array}{l}+0.7 \\
-0.7\end{array}$ \\
\hline $72-83$ & $\begin{array}{l}+9.8 \\
-8.9\end{array}$ & $\begin{array}{l}+2.3 \\
-2.2\end{array}$ & \pm 4.3 & \pm 0.6 & $\begin{array}{l}+0.6 \\
-0.7\end{array}$ \\
\hline $83-96$ & $\begin{array}{l}+9.7 \\
-8.9\end{array}$ & $\begin{array}{l}+2.2 \\
-2.1\end{array}$ & \pm 3.8 & \pm 0.6 & $\begin{array}{l}+0.6 \\
-0.6\end{array}$ \\
\hline $96-110$ & $\begin{array}{l}+9.8 \\
{ }_{-9.0}\end{array}$ & $\begin{array}{l}+2.2 \\
-2.1\end{array}$ & \pm 3.4 & \pm 0.6 & $\begin{array}{l}+0.5 \\
-0.5\end{array}$ \\
\hline $110-127$ & $\begin{array}{l}+10.0 \\
-9.4\end{array}$ & $\begin{array}{l}+2.1 \\
-2.0\end{array}$ & \pm 3.1 & \pm 0.6 & $\begin{array}{l}+0.5 \\
+0.5\end{array}$ \\
\hline $127-146$ & $\begin{array}{l}+10.4 \\
-9.9\end{array}$ & $\begin{array}{l}+2.1 \\
-2.0\end{array}$ & \pm 2.8 & \pm 0.6 & $\begin{array}{l}+0.4 \\
-0.4\end{array}$ \\
\hline $146-169$ & $\begin{array}{l}+11.2 \\
-10.8\end{array}$ & $\begin{array}{l}+2.1 \\
-2.0\end{array}$ & \pm 2.5 & \pm 0.5 & $\begin{array}{l}+0.4 \\
-0.4\end{array}$ \\
\hline $169-195$ & $\begin{array}{l}+12.5 \\
-11.9\end{array}$ & $\begin{array}{l}+2.1 \\
-2.1\end{array}$ & \pm 2.3 & \pm 0.4 & $\begin{array}{l}+0.4 \\
-0.4\end{array}$ \\
\hline $195-224$ & $\begin{array}{l}+14.3 \\
-13.3\end{array}$ & $\begin{array}{l}+2.2 \\
-2.2\end{array}$ & \pm 2.1 & \pm 0.3 & $\begin{array}{l}+0.4 \\
{ }_{-0.3}\end{array}$ \\
\hline $224-259$ & $\begin{array}{l}+16.6 \\
-15.0\end{array}$ & $\begin{array}{l}+2.4 \\
-2.4\end{array}$ & \pm 1.9 & \pm 0.2 & $\begin{array}{l}+0.3 \\
-0.3\end{array}$ \\
\hline $259-298$ & $\begin{array}{l}+19.3 \\
-17.0\end{array}$ & $\begin{array}{l}+2.7 \\
-2.7\end{array}$ & \pm 1.8 & \pm 0.1 & $\begin{array}{l}+0.3 \\
-0.3\end{array}$ \\
\hline $298-344$ & $\begin{array}{r}-22.3 \\
+19.4\end{array}$ & $\begin{array}{l}+2.1 \\
+3.1 \\
-3.2\end{array}$ & \pm 1.6 & \pm 0.1 & $\begin{array}{l}-0.3 \\
+0.3 \\
-0.3\end{array}$ \\
\hline $344-396$ & $\begin{array}{l}+25.7 \\
-22.1\end{array}$ & $\begin{array}{l}+3.7 \\
-3.8\end{array}$ & \pm 1.5 & \pm 0.2 & $\begin{array}{l}+0.3 \\
-0.3\end{array}$ \\
\hline $396-457$ & $\begin{array}{l}+30.7 \\
-25.5\end{array}$ & $\begin{array}{r}+4.5 \\
-4.6\end{array}$ & \pm 1.4 & \pm 0.5 & +0.3 \\
\hline $457-527$ & $\begin{array}{l}+39.5 \\
-29.7\end{array}$ & $\begin{array}{l}+5.5 \\
-5.6\end{array}$ & \pm 1.3 & \pm 1.3 & $\begin{array}{l}+0.3 \\
{ }_{-0.2}\end{array}$ \\
\hline $527-700$ & $\begin{array}{l}+52.6 \\
-37.7\end{array}$ & $\begin{array}{l}+7.4 \\
-7.3\end{array}$ & \pm 1.2 & \pm 4.2 & $\begin{array}{l}+0.3 \\
-0.2\end{array}$ \\
\hline
\end{tabular}

Table C.13: Systematic uncertainties (in percentage) on the measured inclusive jet differential cross section as a function of $p_{T}^{\text {jet }}$, for jets in the region $0.1<\left|y^{j e t}\right|<0.7$ and using $D=0.5$. The different columns follow the discussion in section 4.6. An additional $5.8 \%$ uncertainty on the luminosity is not included. 


\begin{tabular}{|c|c|c|c|c|c|}
\hline \multicolumn{6}{|c|}{ systematic uncertainties $(\%)\left(0.1<\left|y^{j e t}\right|<0.7\right)(D=1.0)$} \\
\hline$p_{T}^{j e t}[\mathrm{GeV} / c]$ & jet energy scale & resolution & unfolding & $p_{T}^{\text {jet }}$-spectra & $\varepsilon$ \\
\hline $54-62$ & $\begin{array}{l}+10.7 \\
-9.4\end{array}$ & $\begin{array}{l}+2.7 \\
-2.7\end{array}$ & \pm 5.6 & \pm 0.4 & $\begin{array}{l}+3.5 \\
-2.9\end{array}$ \\
\hline $62-72$ & $\begin{array}{l}+10.4 \\
-9.3\end{array}$ & +2.6 & \pm 4.9 & \pm 0.4 & $\begin{array}{l}+3.0 \\
-2.6\end{array}$ \\
\hline $72-83$ & $\begin{array}{l}+10.3 \\
-9.2\end{array}$ & +2.4 & \pm 4.2 & \pm 0.4 & ${ }_{-2.4}^{+2.6}$ \\
\hline $83-96$ & $\begin{array}{l}+10.2 \\
-9.2\end{array}$ & +2.3 & \pm 3.7 & \pm 0.4 & $\begin{array}{l}+2.3 \\
+2.2\end{array}$ \\
\hline $96-110$ & $\begin{array}{l}+10.2 \\
-9.3\end{array}$ & $\begin{array}{l}+2.2 \\
-2.2\end{array}$ & \pm 3.2 & \pm 0.4 & $\begin{array}{l}+2.1 \\
{ }_{-2.0}\end{array}$ \\
\hline $110-127$ & $\begin{array}{l}-1.0 .4 \\
+10.4\end{array}$ & $\begin{array}{l}-2.2 \\
+2.1\end{array}$ & \pm 2.8 & \pm 0.4 & $\begin{array}{l}-2.0 \\
+1.9 \\
-1.8\end{array}$ \\
\hline $127-146$ & $\begin{array}{l}+10.8 \\
-10.1\end{array}$ & $\begin{array}{l}+2.0 \\
-2.0\end{array}$ & \pm 2.5 & \pm 0.4 & $\begin{array}{l}+1.7 \\
-1.7\end{array}$ \\
\hline $146-169$ & $\begin{array}{l}+11.5 \\
-10.8\end{array}$ & $\begin{array}{l}+1.9 \\
-1.9\end{array}$ & \pm 2.1 & \pm 0.4 & $\begin{array}{l}+1.6 \\
{ }_{-1.6}\end{array}$ \\
\hline $169-195$ & $\begin{array}{l}+12.6 \\
-11.8\end{array}$ & $\begin{array}{l}+1.9 \\
-2.0\end{array}$ & \pm 1.9 & \pm 0.4 & $\begin{array}{l}+1.5 \\
+1.4\end{array}$ \\
\hline $195-224$ & $\begin{array}{l}+13.9 \\
-13.1\end{array}$ & $\begin{array}{l}+1.9 \\
-2.0\end{array}$ & \pm 1.6 & \pm 0.3 & $\begin{array}{l}+1.4 \\
+1.3\end{array}$ \\
\hline $224-259$ & $\begin{array}{r}-15.1 \\
+14.7\end{array}$ & $\begin{array}{l}+2.1 \\
+2.2\end{array}$ & \pm 1.4 & \pm 0.3 & $\begin{array}{l}-1.3 \\
+1.3\end{array}$ \\
\hline $259-298$ & $\begin{array}{r}+18.0 \\
-16.6\end{array}$ & $\begin{array}{l}+2.4 \\
-2.5\end{array}$ & \pm 1.3 & \pm 0.2 & $\begin{array}{l}+1.3 \\
{ }_{-1.2}\end{array}$ \\
\hline $298-344$ & $\begin{array}{l}-10.0 \\
+20.8 \\
-18.8\end{array}$ & $\begin{array}{l}-2.5 \\
+2.8\end{array}$ & \pm 1.1 & \pm 0.2 & $\begin{array}{l}-1.2 \\
+1.2\end{array}$ \\
\hline $344-396$ & $\begin{array}{l}+24.5 \\
-21.4\end{array}$ & $\begin{array}{l}+3.4 \\
-3.6\end{array}$ & \pm 1.0 & \pm 0.2 & $\begin{array}{l}+1.2 \\
{ }_{-1.1}\end{array}$ \\
\hline $396-457$ & $\begin{array}{l}+30.1 \\
-24.7\end{array}$ & $\begin{array}{r}+4.3 \\
-4.4\end{array}$ & \pm 0.8 & \pm 0.5 & $\begin{array}{l}+1.1 \\
+1.0\end{array}$ \\
\hline $457-527$ & $\begin{array}{l}+38.8 \\
-29.5\end{array}$ & $\begin{array}{l}+5.4 \\
-5.4\end{array}$ & \pm 0.7 & \pm 1.1 & $\begin{array}{l}+1.1 \\
{ }_{-1.0}^{1.0}\end{array}$ \\
\hline $527-700$ & $\begin{array}{l}+49.8 \\
-37.6\end{array}$ & $\begin{array}{l}+7.3 \\
-7.2\end{array}$ & \pm 0.6 & \pm 3.4 & $\begin{array}{l}+1.0 \\
{ }_{-0.9}\end{array}$ \\
\hline
\end{tabular}

Table C.14: Systematic uncertainties (in percentage) on the measured inclusive jet differential cross section as a function of $p_{T}^{\text {jet }}$, for jets in the region $0.1<\left|y^{\text {jet }}\right|<0.7$ and using $D=1.0$. The different columns follow the discussion in section 4.6. An additional 5.8\% uncertainty on the luminosity is not included. 


\section{Bibliography}

[1] R. K. Ellis, W. J. Stirling and B. R. Webber,

"QCD and Collider Physics",

Cambridge Monographs on Particle Physics, Nuclear Physics and Cosmology: 8, Cambridge University Press (1996).

[2] G. Dissertori, I. Knowles and M. Schmelling,

"Quantum Chromodynamics",

Oxford Science Publications (2003).

[3] J.D. Bjorken,

"Asymptotic Sum Rules at Infinite Momentum",

Phys. Rev. 179, 1547 (1969).

[4] E.D. Bloom et al. (SLAC-MIT Collaboration),

"High-Energy Inelastic e-p Scattering at $6^{\circ}$ and $10^{\circ}$ ",

Phys. Rev. Lett. 23, 930 (1969).

[5] H. Deden et al. (Gargamelle Neutrino Collaboration),

"Experimental study of structure functions and sum rules in charge-changing interactions of neutrinos and antineutrinos on nucleons",

Nucl. Phys. B 85, 269 (1975).

[6] H. Abramowicz et al. (CDHS Collaboration),

"Neutrino and antineutrino charged-current inclusive scattering in iron in the energy range $20<E_{\mathrm{v}}<300 \mathrm{GeV}$ ',

Z. Phys. C 17, 283 (1983). 
[7] G. Altarelli and G. Parisi,

"Asymptotic freedom in parton language",

Nucl. Phys. B 126, 298 (1977).

[8] V. N. Gribov and L. N. Lipatov,

" $e^{+} e^{-}$pair annihilation and deep inelastic ep scattering in perturbation theory",

Sov. J. Nucl. Phys. 15, 78 (1972).

[9] J.C. Collins,

"The Problem of Scales: Renormalization and All That", arXiv:hep-ph/9510276 (2006).

[10] MRST Webpage, http://durpdg.dur.ac.uk/hepdata/mrs.html

[11] CTEQ Webpage, http://www.phys.psu.edu/ cteq/

[12] F. Abe et al. (CDF Collaboration),

"Inclusive Jet Cross Section in p anti-p Collisions at $\sqrt{s}=1.8 \mathrm{TeV} "$,

Phys. Rev. Lett. 77, 438 (1996).

[13] H.L. Lai et al.,

"Improved Parton Distributions from Global Analysis of Recent Deep Inelastic Scattering and Inclusive Jet Data",

Phys. Rev. D 55, 1280 (1997).

[14] J. Pumplin et al.,

"New generation of Parton Distribution with Uncertainties from Global QCD Analysis",

JHEP 0207, 012 (2002).

[15] Daniel Stump et al.,

"Inclusive Jet Production, Parton Distributions and the Search for New Physics",

JHEP 0310, 046 (2003).

[16] A. D. Martin et al.,

"Physical gluons and high $-E_{T}$ jets",

Phys. Lett. B 604, 61 (2004).

[17] J. Pumplin et al.,

"Uncertainties of predictions from parton distribution functions. II. The Hessian method",

Phys. Rev. D 65, 014013 (2002). 
[18] W.T. Giele, E.W.N. Glover and David A. Kosower,

"Higher order corrections to jet cross-sections in hadron colliders",

Nucl. Phys. B 403, 633 (1993) [arXiv:hep-ph/9302225].

[19] Pythia Webpage, http://www.thep.lu.se/ torbjorn/Pythia.html

[20] Herwig Webpage, http://hepwww.rl.ac.uk/theory/seymour/herwig/

[21] V.V.Sudakov,

"Vertex parts at very high energies in quantum electrodynamics",

Sov. Phys. J.E.T.P. 3, 65 (1956) (Zh. Eksp. Teor. Fiz. 3087 (1956)).

[22] B. Andersson, G. Gustafson, G. Ingelman and T. Sjöstrand,

"Parton Fragmentation And String Dynamics,"

Phys. Rept. 97, 31 (1983).

[23] T. Sjöstrand,

"Jet Fragmentation of Nearby Partons",

Nucl. Phys. B 248, 469 (1984).

[24] B.R. Webber,

"Simulation of QCD jets including soft gluon interference"

Nucl. Phys.B 238, 492 (1984).

[25] D. Amati and G. Veneziano,

"Preconfinement as a property of perturbative QCD"

Phys. Lett. B 8387 (1979);

[26] G. Hanson et al. (MARK I Collaboration),

"Evidence for Jet Structure in Hadron Production by e+e- annihilation",

Phys. Rev. Lett. 35, 1609 (1975).

[27] $\mathrm{Wu}$ and Zobernig,

"A Method of Three-Jet Analysis in e+e- Annihilation",

Z. Phys. C, Particles and Fields 2, 107 (1979).

[28] J.E. Huth et al.,

"Proceedings of Research Directions For The Decade: Snowmass 1990",

Word Scientific, Singapore, p.134 ( 1992). 
[29] M.H. Seymour,

"Jet Shapes in Hadron Collisions: Higher Orders, Resummation and Hadronization",

Nucl. Phys. B 513, 269 (1998).

[30] S.D. Ellis, Z. Kunszt and D. Soper,

"Jets at hadron colliders at order $\alpha_{s}^{3}$ : A look inside",

Phys. Rev. Lett. 69, 3615 (1992).

[31] W. Bartel et al.,

"Experimental studies on multi-jet production in $e^{+} e^{-}$annihilation at Petra energies",

Z. Phys. C 33, 23 (1986).

[32] S. Catani et al.,

"Longitudinally-Invariant $k_{\perp}$-Clustering Algorithms for Hadron-Hadron Collisions",

Nucl. Phys. B 406, 187 (1993).

[33] S.D. Ellis and D.E. Soper,

"Successive combination jet algorithm for hadron collisions",

Phys. Rev. D 48, 3160 (1993).

[34] T. Affolder et al. (CDF Collaboration),

"Charged Jet Evolution and the Underlying Event in Proton-Antiproton Collisions at $1.8 \mathrm{TeV}$ ", Phys. Rev. D 65, 092002 (2002).

[35] D. Acosta et al. (CDF Collaboration),

"Study of Jet Shapes in Inclusive Jet Production in p anti-p Collisions at s** $(1 / 2)=1.96 \mathrm{TeV}$ ", Phys. Rev. D 71, 112002 (2005).

[36] Fermilab Beam Division, http://www-bd.fnal.gov

[37] Fermilab Beam Division, "Run II Handbook", http://www-bd.fnal.gov/runII/index.html

[38] R. Blair et al.,

"The CDF-II detector: Technical Design Report",

FERMILAB-PUB-96/390-E (1996).

[39] A. Still et al.,

"SVX-II: CDF Run II Silicon Tracking Projects",

Nucl. Instrum. Methods, A 447, 1-8 (2000). 
[40] T.Affolder et al.,

"COT Central Outer Tracker",

Nucl. Instrum. Methods, A 526, 249 (2004).

[41] CDF Tracking Group private conversation;

See also http://www-cdf.fnal.gov/internal/detectors/parameters.html

http://www-cdf.fnal.gov/cdfsim/validation/cot/cotVal.html

[42] D. Acosta et al.,

"A Time-Of-Flight Detector in CDF-II",

Nucl. Instrum. Methods, A 518, 605-608 (2004).

[43] L. Balka et al.,

"The CDF central electromagnetic calorimeter",

Nucl. Instrum. Methods, A 267, 272 (1988).

[44] S. Bertolucci et al.,

"The CDF central and endwall hadron calorimeter",

Nucl. Instrum. Methods, A 267, 301 (1988).

[45] M. Albrow et al.,

"The CDF plug upgrade electromagnetic calorimeter: test beam results",

Nucl. Instrum. Methods, A 480, 524 (2002).

[46] G. Ascoli et al.,

"CDF central muon detector",

Nucl. Instrum. Methods, A 268, 33 (1988).

[47] K. Byrum et al.,

"The CDF forward muon system",

Nucl. Instrum. Methods, A 268, 46 (1988).

[48] D. Acosta et al.,

"The CDF Luminosity Monitor",

Nucl. Instrum. Methods, A 461, 540 (2001).

[49] D. Acosta et al.,

"The Performance of the CDF Luminosity Monitor",

Nucl. Instrum. Methods, A 494, 57 (2002). 
[50] F. Abe et al.,

"Measurement of the anti-proton proton total cross section at $\sqrt{s}=546 \mathrm{GeV}$ and $1800 \mathrm{GeV} "$,

Phys. Rev. D 50, 5550 (1994).

[51] S. Klimenko, J. Konigsberg and T. Liss,

"Averaging the inelastic cross-section measured by CDF and the E811 experiments",

Fermilab-FN-0741 (2003).

[52] S. Jundariani et al.,

"Luminosity Uncertainty for Run 2 up until August 2004",

CDF/ANA/7446 (2005).

[53] H.J. Frisch and P.J. Wilson,

"Trigger tower organization and summing in $\eta-\phi$ space for Run II and beyond", CDF/DOC/TRIGGER/CDFR/2045 (2001).

[54] The XFT System Webpage, http://www.physics.ohio-state.edu/ hughes/xft/

[55] The XTRP Webpage, http://web.hep.uiuc.edu/engin/cdf/xtrp/

[56] I. Vila,

"Performance and First Physics Results of the SVT Trigger at CDFII", arXiv:hep-ph/0307165 (2003).

[57] The Trigger and Datasets Working Group,

"Run II Trigger Table and Datasets Plan", CDF/PHYS/TRIGGER/CDFR/4718 (2001).

[58] A. Bhatti et al. (Jet Energy and Resolution Group),

"Determination of the Jet Energy Scale at the Collider Detector at Fermilab", arXiv:hep-ex/0510047 (2005).

[59] P. Bagnaiaet al. (UA2 Collaboration),

"Measurememts of the jet production properties at the cern anti-p p collider",

Phys. Lett. B 144, 283 (1984).

[60] W.T Giele, E.W.N. Glover and David A. Kosower,

"Higher Order Corrections to Jet Cross Sections in Hadron Colliders",

Nucl. Phys. B 403, 633 (1993) [arXiv:hep-ph/9302225] 
[61] V.M.Abazov et al. (D0 Collaboration),

"The inclusive jet cross section in $p \bar{p}$ collisions at $\operatorname{sqrt}(\mathrm{s})=1.8 \mathrm{TeV}$ using the $\mathrm{kT}$ algorithm", Phys. Lett. B 525, 211 (2002).

[62] Wolfgang Wagner et al.,

"Online Monitorning in the CDF II experiment", EPS HEP 2001 proceedings, FERMILAB-CONF-02-269-E (2001)”

[63] DQM Offline Webpage, http://www-cdf.fnal.gov/internal/dqm/dqm_offline.html

[64] Good Run List Webpage, http://www-cdf.fnal.gov/internal/dqm/goodrun/good.html 